\title{
Oxygen homeostasis and stress in the ductus arteriosus
}

Citation for published version (APA):

van der Sterren, S. (2016). Oxygen homeostasis and stress in the ductus arteriosus: studies in the chicken embryo model. [Doctoral Thesis, Maastricht University]. Maastricht University. https://doi.org/10.26481/dis.20160309ss

Document status and date:

Published: 01/01/2016

DOI:

10.26481/dis.20160309ss

Document Version:

Publisher's PDF, also known as Version of record

\section{Please check the document version of this publication:}

- A submitted manuscript is the version of the article upon submission and before peer-review. There can be important differences between the submitted version and the official published version of record.

People interested in the research are advised to contact the author for the final version of the publication, or visit the DOI to the publisher's website.

- The final author version and the galley proof are versions of the publication after peer review.

- The final published version features the final layout of the paper including the volume, issue and page numbers.

Link to publication

\footnotetext{
General rights rights.

- You may freely distribute the URL identifying the publication in the public portal. please follow below link for the End User Agreement:

www.umlib.nl/taverne-license

Take down policy

If you believe that this document breaches copyright please contact us at:

repository@maastrichtuniversity.nl

providing details and we will investigate your claim.
}

Copyright and moral rights for the publications made accessible in the public portal are retained by the authors and/or other copyright owners and it is a condition of accessing publications that users recognise and abide by the legal requirements associated with these

- Users may download and print one copy of any publication from the public portal for the purpose of private study or research.

- You may not further distribute the material or use it for any profit-making activity or commercial gain

If the publication is distributed under the terms of Article $25 \mathrm{fa}$ of the Dutch Copyright Act, indicated by the "Taverne" license above, 


\section{Oxygen homeostasis and stress in the ductus arteriosus Studies in the chicken embryo model}

\section{Dissertation}

to obtain the degree of Doctor at Maastricht University, on the authority of the Rector Magnificus,

Prof. dr. L.L.G. Soete

in accordance with the decision of the Board of Deans, to be defended in public on Wednesday 9th of March 2016, at 16.00 hours

$$
\text { by }
$$

Saskia Anna Josepha Wilhelmina van der Sterren 


\section{Supervisors:}

Prof. dr. L.J.I. Zimmermann

Prof. dr. A.L. Cogolludo Torralba (Complutense University of Madrid, Madrid)

\section{Co-supervisor:}

Dr. E.I.V. Villamor Zambrano

\section{Assessment committee:}

- Prof. dr. B.W.W. Kramer (chairman)

- Prof. dr. A. Bast

- Prof. dr. R.I. Clyman (UCSF Medical Centre, San Francisco)

- Prof. dr. T. Delhaas

- Prof. dr. I.K.M. Reiss (Erasmus MC-Sophia Kinderziekenhuis, Rotterdam)

Financial support by the Dutch Heart Foundation for the publication of this thesis is gratefully acknowledged. 


\section{Contents}

Abbreviations $\quad 4$

$\begin{array}{ll}\text { Justification, aim, and outline } & 7\end{array}$

Chapter 1 Prenatal cardiovascular shunts in mammals and chicken 15

Chapter 2 Maturation of $\mathrm{O}_{2}$ sensing and signaling in the chicken ductus arteriosus

Chapter $3 \quad$ Morphological and functional alterations of the ductus arteriosus in a chicken model of hypoxia-induced fetal growth retardation

Chapter 4 Prenatal exposure to hyperoxia modifies the thromboxane prostanoid receptor-mediated response to $\mathrm{H}_{2} \mathrm{O}_{2}$ in the ductus arteriosus of the chicken embryo

Chapter 5 Vasoactivity of the gasotransmitters hydrogen sulfide and carbon monoxide in the chicken ductus arteriosus

Chapter 6 Contractile effects of 15- $\mathrm{E}_{2 \mathrm{t}}$-isoprostane and 15- $\mathrm{F}_{2 \mathrm{t}}{ }^{-}$ isoprostane on chicken embryo ductus arteriosus

Chapter $7 \quad$ Effects of sex and estrogen on chicken ductus arteriosus reactivity

Chapter 8 Summary, general discussion and future perspectives

Chapter $9 \quad$ Samenvatting

Chapter $10 \quad$ Valorization

Dankwoord (acknowledgements) 


\section{Abbreviations}

$-d$

4-AP

$\mathrm{ACh}$

AF

AOA

AoDA/aoDA

BK $K_{C a}$ channel

Caı channel

CAM

CBS

$\mathrm{CO}$

CORM

COX

CSA

CSE

$C$ T method

CYP450

DA

DASMC

DCF

DHE

DMSO

DPN

DV

Ex

EDHF

EDRF

EDTA

EGTA

eNOS

ER

ET-1

ETC

FA

FO

GAPDH

$\mathrm{HO}$ days

4-aminopyridine

acetylcholine

atrial foramina

amino-oxyacetic acid (CBS inhibitor)

aortic part of the ductus arteriosus

large-conductance $\mathrm{Ca}_{2}$-activated potassium channel

L-type calcium ion channel

chorioallantoic membrane

cystathionine- $\beta$-synthase

carbon monoxide

CO releasing molecule

cyclooxygenase

cross sectional area

cystathionine- $\gamma$-lyase

comparative threshold cycle method

cytochrome P450

ductus arteriosus

ductus arteriosus smooth muscle cells

2',7'-dichlorofluorescein

dihydroethidium

dimethylsulfoxide

2,3-bis(4-hydroxyphenyl)-propionitrile

ductus venosus

day $x$ of the 21 day chicken embryo incubation period

endothelium-derived growth factor

enothelium-derived relaxing factor

ethylene diamine tetraacetic acid

ethylene glycol tetraacetic acid

endothelial NOS

estrogen receptor

endothelin-1

electron transport chain

femoral artery

foramen ovale

glyceraldehyde 3-phospate dehydrogenase

heme oxygenase 


\begin{tabular}{|c|c|}
\hline $\mathrm{H}_{2} \mathrm{O}_{2}$ & hydrogen peroxide \\
\hline $\mathrm{H}_{2} \mathrm{~S}$ & hydrogen sulfide \\
\hline $\mathrm{IK}_{\mathrm{Ca}}$ channel & $\begin{array}{l}\text { intermediate-conductance } \mathrm{Ca}_{2} \text {-activated potassium } \\
\text { channel }\end{array}$ \\
\hline IsoP & isprostane \\
\hline IUGR & intra uterine growth retardation \\
\hline IVC & inferior vena cava \\
\hline KRB buffer & Krebs-Ringer bicarbonate buffer \\
\hline $\mathrm{K}_{\mathrm{v}}$ channel & voltage operated potassium channel \\
\hline KATP channel & ATP-sensitive potassium channel \\
\hline LA & left atrium \\
\hline L-NAME & NG-nitro-L-arginine methyl ester (NOS inhibitor) \\
\hline LPA & left pulmonary artery \\
\hline LV & left ventricle \\
\hline NE & norepinephrine \\
\hline NEC & necrotizing enterocolitis \\
\hline NO & nitric oxide \\
\hline NOS & nitric oxide synthetase \\
\hline ODQ & $\begin{array}{l}\text { 1H-[1,2,4]oxadiazolo[4,3-a]quinoxalin-1-one } \\
\text { (sGC inhibitor) }\end{array}$ \\
\hline PA & pulmonary artery \\
\hline PAF & platelet activating factor \\
\hline pDA & pulmonary part of the ductus arteriosus \\
\hline pDASMC & $\begin{array}{l}\text { smooth muscle cells of pulmonary part of ductus } \\
\text { arteriosus }\end{array}$ \\
\hline PDA & persistently patent ductus arteriosus \\
\hline PEG & polyethylene glycol \\
\hline$P G$ & prostaglandin \\
\hline $\mathrm{PGE}_{2}$ & prostaglandin $E_{2}$ \\
\hline $\mathrm{PGH}_{2}$ & prostaglandin $\mathrm{H}_{2}$ \\
\hline $\mathrm{PGI}_{2}$ & prostaglandin $\mathrm{I}_{2}$ \\
\hline Phe & phenylephrine \\
\hline PKG & protein kinase $\mathrm{G}$ \\
\hline PPG & D,L-propargylglycine (CSE inhibitor) \\
\hline PPT & 4,4',4"'-(4-propyl-['H]pyrazole-1,3,5-triyl)trisphenol \\
\hline PSS & physiological salt solution \\
\hline PulmDA & pulmonary part of the ductus arteriosus \\
\hline PVDF & polyvinylidene difluoride \\
\hline PVL & periventricular leukomalacia \\
\hline
\end{tabular}


RA

ROS

RPA

$R Q$

RV

SD

SEM

sGC

SKca channel

SNP

SOD

SVC

TEA

TX

$\mathrm{TXA}_{2}$

TP receptor

vOCC

ZnPP IX right atrium

reactive oxygen species

right pulmonary artery

relative quantitation

right ventricle

standard deviation

standard error of means

soluble guanylate cyclase

small-conductance $\mathrm{Ca}_{2}$-activated potassium channel

sodium nitroprusside

superoxide dismutase

superior vena cava

tetraethylammonium

thromboxane

thromboxane $\mathrm{A}_{2}$

thromboxane prostanoid receptor

voltage operated $\mathrm{Ca}^{2+}$ channels

zinc protoporphyrin IX 


\section{Justification, aim and outline}


The ductus arteriosus (DA) is a fetal shunt that connects the pulmonary artery to the aorta, providing a pulmonary-to-systemic diversion that allows right ventricular output to bypass the nonventilated fetal lungs [1]. Patency of the DA is necessary for normal cardiovascular and pulmonary vascular development of the fetus and is regulated by a fine balance of constricting and dilating forces [1, 2]. The main factors maintaining patency of the DA in the fetus are the relatively hypoxic environment and the high levels of circulating prostaglandin $(P G) E_{2}$, and locally produced $\mathrm{PGE}_{2}$ and $\mathrm{PGI}_{2}[1,3,4]$.

Postnatal closure of the DA is an important step in normal cardio-pulmonary transition and occurs in two phases. Initially, the increase in arterial $\mathrm{O}_{2}$ tension at birth and the decrease in PG concentration and activity are key factors stimulating DA constriction, which functionally limits luminal blood flow $[1,2,4$, 5]. After this functional occlusion has occurred, hypoxia and nutrition deprivation of the medial layer of the DA results in the production of inflammatory mediators and growth factors. These compounds subsequently induce fibrosis, resulting in permanent anatomic closure of the DA $[1,2,4,5]$.

Premature birth interrupts development of DA contractile mechanisms and the normal maturation process, leaving the immature DA more susceptible to remain open after birth. This is partially explained by decreased sensitivity to oxygen, diminished responsiveness to prostaglandin withdrawal at birth, increased levels of PGs, nitric oxide (NO) and other vasodilatory stimuli, and immaturity of the structural apparatus necessary for the constriction and obliteration of the DA lumen [1, 3, 6-10]. Achieving definitive, anatomical closure is challenged by the lower intrinsic tone of the premature DA and the increased sensitivity to vasodilators hindering functional closure, explaining at least partly why premature infants have a high rates of reopening of the DA [8]. Therefore, preterm neonates, and more particularly those with gestational age $<30$ weeks, and birth weight $<1500$ grams, are at increased risk for having a persistently patent DA (PDA) $[4,11]$. The resulting left-to-right shunt and hyperperfusion of the pulmonary vascular bed often result in decreased lung compliance, increased need for mechanical ventilation and altered postnatal nutrition and growth $[4,11]$. This situation has been classically related to an increased the risk of adverse outcomes such as chronic lung disease [12], pulmonary hemorrhage [13], renal hypoperfusion [7] or necrotizing enterocolitis [14]. However, although numerous studies have examined the association of various neonatal morbidities in the presence of a PDA and their occurrence after treatment, the cause-and-effect relationships remain unclear [15]. Therefore, 
over recent years, the clinical approach to PDA in the premature neonate has been the subject of intensive reevaluation $[16,17]$.

The hope for future targeted therapies and for the prevention of PDA requires knowledge of the fundamental mechanisms controlling its development and pathogenesis [18]. Several mammalian models have helped to unravel the biology and pathobiology of the DA and to understand the factors responsible for its closure or patency after birth [19]. However, these models are technically complex and experimental manipulations affect both the mother and the fetus. Therefore, there is a need for additional models, addressing these limitations [19]. In the last years, the chicken embryo has emerged as a suitable model for the study of DA vascular biology [19-21]. The chicken embryo actually has the longest continuous history as an experimental model for developmental biology studies and has also contributed major concepts to immunology, genetics, virology, cancer and cell biology [22]. As the chicken embryo develops outside the mother, it allows for study of cardiovascular responses without the influence of maternal physiology (i.e. hormonal, metabolic, hemodynamic) [19, 20, 23-25]. For the same reason it is relatively easy to monitor and maintain prenatal conditions or to manipulate them. Our group and another laboratory have characterized the developmental changes in the responsiveness of the chicken DA to the vasoactive mediators involved in the control of fetal patency and postnatal closure of mammalian DA. These include $\mathrm{O}_{2}, P G s$, endothelin-1, catecholamines, carbon dioxide and nitric oxide (NO) [20, 26-32]. These studies provide supportive evidence for a conserved mechanism for $\mathrm{O}_{2}$ sensing/ signaling in mammalian and non-mammalian DA [20, 21, 29, 30]. Moreover, similarly to the mammalian DA [4, 33], the responsiveness of chicken DA to $\mathrm{O}_{2}$ and other vasoconstrictors is developmentally enhanced [20, 28, 34]. On the other hand, the response to relaxant agents decreased with development [26, 29].

The aim of the present thesis was to delve into the developmental biology of the DA, using the chicken embryo as model. Particularly, we further investigated the mechanisms involved in the response of the chicken DA to oxygen and we analyzed the effects of changing the oxygen tension during incubation on the development of chicken DA. In addition, we studied vasoactive mediators, such as $\mathrm{H}_{2} \mathrm{~S}$, isoprostanes or estrogen that had not yet been investigated in the DA of any species. 
In chapter 1 we discuss the comparative biology of the mammalian and the chicken ductus arteriosus. In spite of some anatomical and functional differences, the chicken DA appears to be a suitable model to study DA reactivity.

In chapter 2 we investigate the pathways by which the chicken DA's response to oxygen is mediated. The proposed mechanism for oxygen sensing and signaling in mammalian DA, i.e. the mitochondrial electron transport chain as oxygen sensor, $\mathrm{H}_{2} \mathrm{O}_{2}$ as mediator and voltage-gated potassium channels as effector, modulated by calcium sensitization by (in)activation of Rho-kinase, also applies to normoxic contraction in our chicken DA model.

DA development takes place under relatively hypoxic conditions. In chapters 3 and 4 we examined whether changing these prenatal conditions, i.e. making them more hypoxic or hyperoxic, alters the developmental trajectory of the DA.

Besides nitric oxide (NO) and carbon monoxide (CO), hydrogen sulfide $\left(\mathrm{H}_{2} \mathrm{~S}\right)$ is a third gaseous messenger that may play a role in controlling vascular tone and has been proposed to serve as an $\mathrm{O}_{2}$ sensor. However, whether $\mathrm{H}_{2} \mathrm{~S}$ is vasoactive in the ductus arteriosus (DA) has not yet been studied. The mechanical responses of the chicken DA to these gaseous messengers are examined in chapter 5 .

Chapter 6 is dedicated to the role of isoprostanes in the control of chicken DA tone. Isoprostanes are prostaglandin-like compounds produced nonenzymatically by free radical-catalyzed peroxidation of arachidonate. While PGs play a major role in DA homeostasis, the putative role of isoprostanes had not yet been studied.

In Chapter 7, we analyzed the putative sexual dimorphism and the influence of sex hormones on the reactivity of the chicken DA. Sex hormones have an important influence on cardiovascular physiology and pathophysiology and sex differences in vascular reactivity have been widely demonstrated in several vascular beds but not in the DA. 


\section{References}

1. Smith, G.C., The pharmacology of the ductus arteriosus. Pharmacol Rev, 1998. 50(1): p. 35-58.

2. Hajj, H. and J.M. Dagle, Genetics of patent ductus arteriosus susceptibility and treatment. Semin Perinatol, 2012. 36(2): p. 98-104.

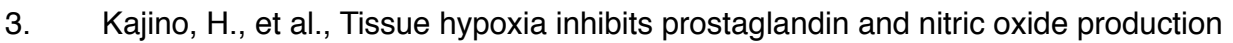
and prevents ductus arteriosus reopening. Am J Physiol Regul Integr Comp Physiol, 2000. 279(1): p. R278-86.

4. Clyman, R.I., Mechanisms regulating the ductus arteriosus. Biology of the Neonate, 2006. 89(4): p. 330-335.

5. Coceani, F. and B. Baragatti, Mechanisms for ductus arteriosus closure. Semin Perinatol, 2012. 36(2): p. 92-7.

6. Clyman, R.I., Ductus arteriosus: developmental response to endogenous prostaglandins, oxygen, and indomethacin. Adv Prostaglandin Thromboxane Res, 1980. 7: p. 887-90.

7. Hammerman, C., Patent ductus arteriosus. Clinical relevance of prostaglandins and prostaglandin inhibitors in PDA pathophysiology and treatment. Clin Perinatol, 1995. 22(2): p. 457-79.

8. Kajino, $\mathrm{H}$., et al., Factors that increase the contractile tone of the ductus arteriosus also regulate its anatomic remodeling. Am J Physiol Regul Integr Comp Physiol, 2001. 281(1): p. R291-301.

9. Van Overmeire, B. and S. Chemtob, The pharmacologic closure of the patent ductus arteriosus. Semin Fetal Neonatal Med, 2005. 10(2): p. 177-84.

10. Reese, J., Death, dying, and exhaustion in the ductus arteriosus: prerequisites for permanent closure. Am J Physiol Regul Integr Comp Physiol, 2006. 290(2): p. R357-8.

11. Koch, J., et al., Prevalence of spontaneous closure of the ductus arteriosus in neonates at a birth weight of 1000 grams or less. Pediatrics, 2006. 117(4): p. 1113-21.

12. Rojas, M.A., et al., Changing trends in the epidemiology and pathogenesis of neonatal chronic lung disease. J Pediatr, 1995. 126(4): p. 605-10.

13. Kluckow, M., et al., Prostacyclin concentrations and transitional circulation in preterm infants requiring mechanical ventilation. Arch Dis Child Fetal Neonatal Ed, 1999. 80(1): p. F34-7.

14. Cotton, R.B., et al., Medical management of small preterm infants with symptomatic patent ductus arteriosus. J Pediatr, 1978. 92(3): p. 467-73. 
15. Jaleel, M.A. and C.R. Rosenfeld, Patent ductus arteriosus and intraventricular hemorrhage: a complex association. J Pediatr, 2013. 163(1): p. 8-10.

16. Clyman, R.I., J. Couto, and G.M. Murphy, Patent ductus arteriosus: are current neonatal treatment options better or worse than no treatment at all? Semin Perinatol, 2012. 36(2): p. 123-9.

17. Hammerman, C., A. Bin-Nun, and M. Kaplan, Managing the patent ductus arteriosus in the premature neonate: a new look at what we thought we knew. Semin Perinatol, 2012. 36(2): p. 130-8.

18. Stoller, J.Z., et al., Current Perspectives on Pathobiology of the Ductus Arteriosus. J Clin Exp Cardiolog, 2012. S8:001.

19. Sutendra, G. and E.D. Michelakis, The chicken embryo as a model for ductus arteriosus developmental biology: cracking into new territory. Am J Physiol Regul Integr Comp Physiol, 2007. 292(1): p. R481-4.

20. Agren, P., et al., Ontogeny of chicken ductus arteriosus response to oxygen and vasoconstrictors. Am J Physiol Regul Integr Comp Physiol, 2007. 292(1): p. R485-96.

21. Dzialowski, E.M., et al., Prenatal cardiovascular shunts in amniotic vertebrates. Respir Physiol Neurobiol, 2011. 178(1): p. 66-74.

22. Stern, C.D., The chick; a great model system becomes even greater. Dev Cell, 2005. 8(1): p. 9-17.

23. Ruijtenbeek, K., et al., Chronic hypoxia stimulates periarterial sympathetic nerve development in chicken embryo. Circulation, 2000. 102(23): p. 2892-7.

24. le Noble, F.A., et al., Contractile and relaxing reactivity in carotid and femoral arteries of chicken embryos. Am J Physiol Heart Circ Physiol, 2000. 278(4): p. H1261-8.

25. Ruijtenbeek, K., J.G. De Mey, and C.E. Blanco, The chicken embryo in developmental physiology of the cardiovascular system: a traditional model with new possibilities. Am J Physiol Regul Integr Comp Physiol, 2002. 283(2): p. R549-50; author reply $\mathrm{R} 550-1$.

26. Agren, P., et al., Developmental changes in endothelium-dependent relaxation of the chicken ductus arteriosus. J Physiol Pharmacol, 2008. 59(1): p. 55-76.

27. Belanger, C., et al., Morphological changes in the chicken ductus arteriosi during closure at hatching. Anat Rec (Hoboken), 2008. 291(8): p. 1007-15.

28. Greyner, H. and E.M. Dzialowski, Mechanisms mediating the oxygen-induced vasoreactivity of the ductus arteriosus in the chicken embryo. Am J Physiol Regul Integr Comp Physiol, 2008. 295(5): p. R1647-59. 
29. Agren, P., et al., Developmental changes in the effects of prostaglandin E2 in the chicken ductus arteriosus. J Comp Physiol B, 2009. 179(2): p. 133-43.

30. Copeland, J. and E.M. Dzialowski, Effects of hypoxic and hyperoxic incubation on the reactivity of the chicken embryo (Gallus gallus) ductus arteriosi in response to catecholamines and oxygen. Exp Physiol, 2009. 94(1): p. 152-61.

31. Moonen, R.M., et al., Response of chicken ductus arteriosus to hypercarbic and normocarbic acidosis. Neonatology, 2010. 98(1): p. 47-56.

32. Schuurman, M.J. and E. Villamor, Endothelium-dependent contraction induced by acetylcholine in the chicken ductus arteriosus involves cyclooxygenase-1 activation and TP receptor stimulation. Comp Biochem Physiol A Mol Integr Physiol, 2010. 157(1): p. 28-34.

33. Thebaud, B., et al., Oxygen-sensitive Kv channel gene transfer confers oxygen responsiveness to preterm rabbit and remodeled human ductus arteriosus: implications for infants with patent ductus arteriosus. Circulation, 2004. 110(11): p. 1372-9.

34. Flinsenberg, T.W., et al., Effects of sex and estrogen on chicken ductus arteriosus reactivity. Am J Physiol Regul Integr Comp Physiol, 2010. 298(5): p. R1217-24.

35. Moonen, R.M., et al., Response of Chicken Ductus Arteriosus to Hypercarbic and Normocarbic Acidosis. Neonatology, 2009. 98(1): p. 47-56. 


\section{Chapter 1}

\section{Prenatal cardiovascular shunts in mammals and chicken}

Adapted from:

Prenatal cardiovascular shunts in amniotic vertebrates.

EM Dzialowski, T Sirsat, S van der Sterren, E Villamor Respir Physiol Neurobiol. 2011 Aug 31;178(1):66-74 


\subsection{Introduction}

The prenatal developing cardiovascular system of amniotic vertebrates is unique, complex and differs from the neonatal and adult circulation. A number of functional prenatal vascular shunts have evolved to deal with the non-ventilation of the lungs, the source of nutrients (placenta and yolk sac), and the reliance on the embryonic gas exchanger; the chorioallantoic membrane (CAM) in oviparous and the placenta in placental mammals. These vascular shunts provide preferential distribution of oxygen to various organs and also function as a partial bypass of the lungs. In this chapter, we first examine the comparative anatomy and patterns of blood flow of the prenatal shunts in mammals and chicken. We then discuss the regulation and closure of the fetal shunts.

\subsection{Comparative anatomy of prenatal shunts}

\subsubsection{Mammalian shunts}

The developing mammalian fetus has a number of functional venous and arterial shunts (Figure 1.1A). The ductus venosus (DV) is a shunt that diverts oxygenated blood returning to the fetus from the umbilical vein away from the liver and to the inferior vena cava and eventually the foramen ovale and left atrium. A functional DV has been described in fetuses of humans, mice, rats, dogs, cats, and many other mammals [1]. In pig fetuses, the DV disappears during early prenatal life [1]. The two other shunts, the ductus arteriosus (DA) and foramen ovale are involved in a right-to-left shunt away from the right side of the heart and pulmonary circuit and towards the systemic circuit. The foramen ovale, arises from a bifurcation of the inferior vena cava and DV returning to the heart [2]. One branch feeds the right atrium and the second branch forms the foramen ovale into the left atrium. It functions as a wind sleeve to divert venous return to the left side of the heart. The third shunt, the DA, is a fetal vessel derived from the sixth aortic arch that connects the pulmonary artery with the aorta. This provides a shunt of right cardiac output away from the nonventilating lungs and to the descending aorta, the placenta, and lower body. 


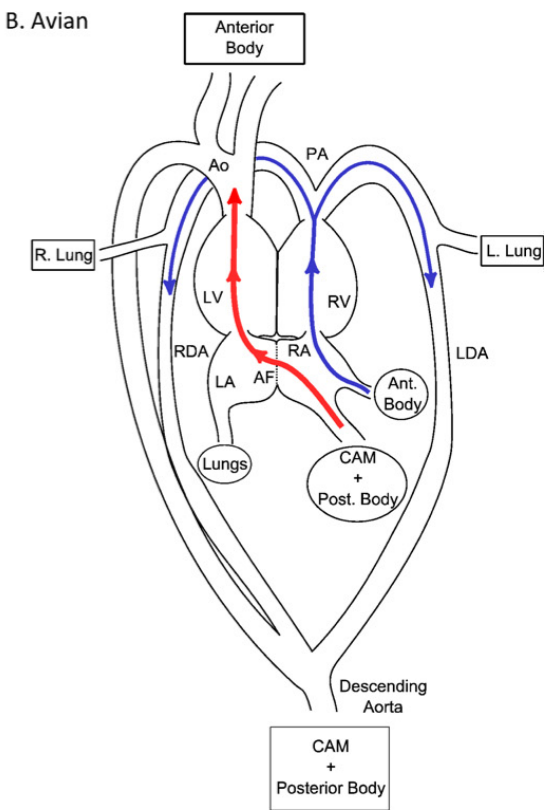

Fig. 1.1. Schematic representations of the prenatal circulation in (A) the mammalian fetus and (B) the avian embryo. Paths with arrows represent the right-to-left shunts in each group. Red paths represent oxygenated blood and blue paths represent less well oxygenated blood. (A) The foramen ovale (FO) and the ductus arteriosus (DA) provide a right-to-left shunt in the mammals. (B) The atrial foramina (AF) and two DA provide a right-to-left shunt in the birds.

$A F$, atrial foramina; CAM, chorioallantoic membrane; $D A$, ductus arteriosus; RDA, right ductus arteriosus; LDA, left ductus arteriosus; DV, ductus venosus; FO, foramen ovale; IVC, inferior vena cava; SVC, superior vena cava; RA, right atrium; LA left atrium; RV, right ventricle; LV, left ventricle; PA, pulmonary artery; RPA, right pulmonary artery; LPA, left pulmonary artery; R. Lung, right lung; L. Lung, left lung.

\subsubsection{Chicken Shunts}

The chicken embryo has two of the embryonic shunts found in mammals during development (Figure 1.1B). As with the mammalian fetus, the avian embryo has a right-to-left shunt through the DA. The mammalian fetus has a single DA connecting the aorta and pulmonary artery. In contrast, the avian embryo has two DAs present during embryonic development. The right and left DA branch from the right and left pulmonary artery and join with the aorta to form the descending aorta, supplying blood to the lower body and CAM. There is an intra-cardiac shunt in the embryonic avian heart through perforations or foramina in the wall separating the right and left atrium. This atrial shunt functions in much the same way as the foramen ovale in the mammalian fetus, allowing posterior venous return to flow from the right atria into the left atria [3]. 
While the DV has been described in early chicken embryos, it disappears during development [4]. Thus, a DV is present during the first seven days of chick development as liver and hepatic circulation begins to develop. By the end of the seventh day, the DV is absorbed by the developing liver [4] and is not present in later stages of the chicken embryo [3]. Blood returning from the CAM via the allantoic vein is directed back to the heart through the left hepatic vein [3]. Absence of a DV during later stages of embryonic development may in part be due to the source of nutrients in the avian embryo, whose liver receives nutrient rich blood from the yolk sac through the mesenteric and vitelline veins. In comparison, the liver of the mammalian fetus receives nutrient rich blood from the placenta through the umbilical vein.

\subsection{Blood flow and oxygen saturation}

\subsubsection{Mammalian}

During fetal stages, the circulation patterns of developing mammals are relatively well characterized. Because of the complete separation of the adult heart and pulmonary and systemic circulation, the right and left cardiac output are equal. In contrast, total cardiac output of the developing mammalian fetus is unequally divided between the right ventricle and left ventricle [5]. In human and sheep fetuses, right ventricular cardiac output accounts for between 53 and $60 \%$ of total cardiac output, while left ventricular output ranges between 40 and $47 \%$ of the total cardiac output $[6,7]$. From the blood ejected by the right ventricle, only $10-15 \%$ passes through the lungs, the remaining $85-90 \%$ passing through the DA to the descending aorta [8]. In addition, due to the right-to-left shunt of blood through the DA, blood flow returning from the lungs to the left atria is low and makes only a small contribution to left ventricular output. The major contribution of left ventricular output comes from the foramen ovale [7, 9]. Mielke and Benda [7] found that foramen ovale blood flow contributed $76 \%$ of left ventricular output and $33 \%$ to $34 \%$ of combined cardiac output in human and lamb fetuses respectively. Foramen ovale blood flow is influenced by changes in pulmonary vascular resistance [8]. Oxygenated blood from the umbilical vein that has bypassed the liver through the DV makes up a large proportion of venous return to the heart via the foramen ovale. The partial pressure of oxygen $\left(\mathrm{PO}_{2}\right)$ in the umbilical vein is around $4.7 \mathrm{kPa}$ and fetal blood is $80-90 \%$ saturated [10]. The proportion of umbilical blood shunted away from the liver and through the DV during control conditions ranges between 34 to $70 \%$ [1]. In several mammalian species, including humans, fetal inferior venous 
return is arranged in a manner by which blood from the DV and hepatic veins preferentially enters the left heart via the foramen ovale to supply the coronary arteries and brain with well oxygenated blood [11]. Thus, the $\mathrm{PO}_{2}$ of blood supplying the heart, brain, head, and neck is 0.5 to $0.7 \mathrm{kPa}$ higher than that of blood in the descending aorta. The shunt of blood directed to the liver is a greater proportion of umbilical return in humans than other mammals [12, 13]. Shunting through the DV increases upon exposure to maternal hypoxia $[12,14]$.

\subsubsection{Chicken}

Shunting patterns in the chicken embryo have been less well characterized because it has been more difficult to measure shunting in ovo. Unlike mammals, where the DA is located superior to the heart and connects the aorta and pulmonary artery near their exit from the heart, chicken DAs are located deep in the thoracic cavity, dorsal to the esophagus and heart. This location deep within the body makes it difficult to place blood flow probes around the DAs for in situ blood flow measurements. Therefore, studies of embryonic blood flow patterns in bird embryos have typically involved injecting dyes or microspheres into the allantoic vein and examining where they are trapped.

Using dye injection, White [3] examined flow patterns of anterior and posterior venous return through the late stage embryonic chicken heart. The posterior venous channel receives oxygenated blood returning from the allantoic vein. As with the mammalian foramen ovale, the majority of blood (73.5\%; [3]) entering the right atria from the posterior venous channel is shunted through the intraatrial perforations and into the left ventricle and aortic arch. The remaining blood, returning from the posterior venous channel flows to the right ventricle. Deoxygenated blood returning from the head region via the anterior vena cava preferentially flows through the right side of the heart and into the pulmonary arteries and DA (Figure 1.1B). It is expected that the majority of blood flow in the pulmonary arteries by-passes the lungs and is shunted to the dorsal aorta through the DA [15]. The diameter of the pulmonary arteries at the junction with the DA is smaller than that of the DA, supporting this view of preferential flow to the DA. A high percentage of the blood from the DA flows to the CAM for oxygenation.

One of the differences between the mammalian fetus and avian embryo is the timing of changes in the vascular shunts at birth and hatching (Figure 1.2). Because mammals lose their fetal respiratory organ and circulation as soon as they are born, the shunts within the mammalian neonate must close rapidly upon birth and initiation of lung ventilation. However, the chicken embryo relies on both their lungs and CAM during the longer hatching process (Figure 1.2A). 
Using microspheres, Rahn et al. [16] showed that there is an increase in blood flow to the lungs and a decrease in blood flow to the CAM with hatching. Prior to the initiation of hatching, very few microspheres were found in the lung of day 17-19 chicken embryos. As White [3] found, the majority of venous return from the allantoic vein encounters a right-to-left shunt through the intra-atrial foramina and the DA. Tazawa and Takenaka [15] estimated that approximately $60 \%$ of the posterior venous return follows the right-to-left shunt through these perforations. During hatching, the embryo first internally pips the inner membrane and begins to respire on hypoxic gas in the air cell. At this point, the embryo relies on both the CAM and the lungs for oxygen uptake. This suggests that flow through the DA is decreasing as flow through the pulmonary arteries increases. During internal pipping, the embryo is able to alter the right-to-left shunt in response to hypoxic or hyperoxic exposure at either the air cell or the CAM [17]. Flow to both the CAM and brain in relation to the lungs increased after changing the air cell gas from the normal value of $10 \% \mathrm{O}_{2}$ to more hypoxic $5 \% \mathrm{O}_{2}$. The opposite response was seen when the air cell gas was made hyperoxic. These changes in blood flow pattern mirror the changes in the contribution to $\mathrm{O}_{2}$ uptake at the lungs and the CAM [17]. These changes suggest that flow through the intra-atrial foramina and DA changes in response to alterations in $\mathrm{O}_{2}$ availability at the lungs, most likely through decreases in resistance of the pulmonary circuit and increased resistance in the systemic arterial circuit as CAM circulation gradually decreases. Between 8 and 24 hours later, the embryo externally pips the eggshell and begins to respire normoxic air. At this point, blood flow to the CAM approaches 0 and approximately $90 \%$ of the microspheres were found in the lungs. Upon hatching all of the microspheres were found in the lungs. These changes in blood flow patterns to the lungs, $\mathrm{CAM}$, and embryo mirror those of the changes in DA lumen diameter (Figure $1.2 \mathrm{~B} ;[18])$. 
Fig. 1.2.

A

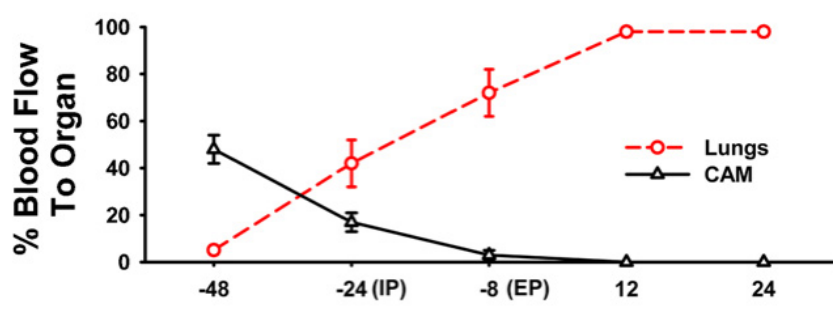

B

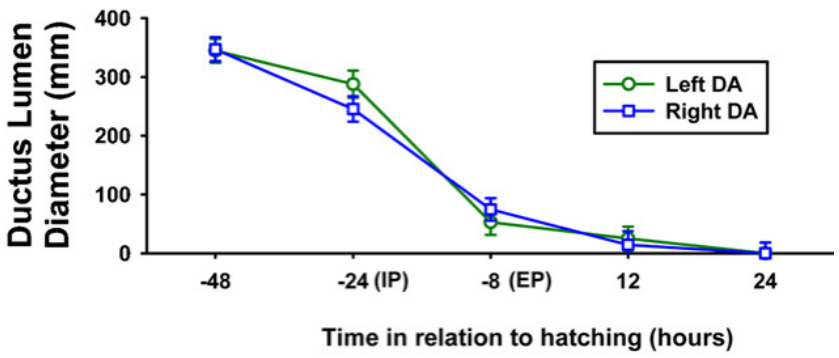

C

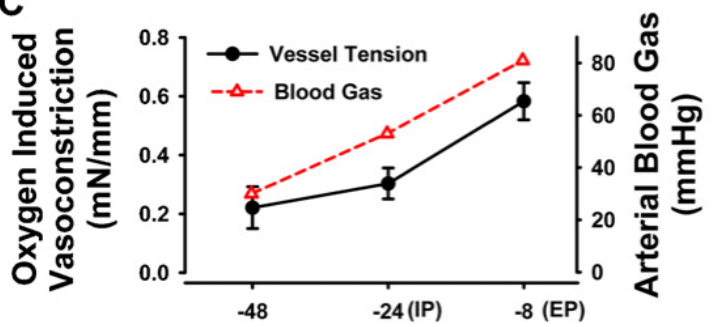

Time in relation to hatching (hours)
Cardiovascular changes in the chicken embryo during hatching. (A) Right atrial blood flow to the lungs and the CAM prior to and during hatching [16]. (B) Lumen diameter of the right and left DA in the hatching chicken [18]. (C) $\mathrm{O}_{2}$ induced contraction of the right DA [18] and arterial blood gas tensions [19] during hatching.

In response to hypoxic exposure, the chicken embryo is able to redistribute blood flow among the different organs. As with mammals, there is a general increase in flow to the heart and brain of the embryo during anoxic exposure after day 14 of incubation [20, 21]. Associated with the increase in heart and brain blood flow is a decrease in flow to the liver and intestines. Flow to the CAM increases significantly after anoxic exposure during days 14 to 16 . Changes in response to less drastic $\mathrm{O}_{2}$ levels are unknown. 


\subsection{DA regulation and closure}

\subsubsection{Mammalian DA}

The physiology and closure of the DA has been best studied in mammalian models. The DA is distinguished from the surrounding vasculature by its embryologic derivation from the sixth aortic arch, the contribution of migratory neural crest cells, and exquisite sensitivity to oxygen tension [22, 23]. In mammals, low oxygen tension, high levels of circulating prostaglandin $(P G) E_{2}$, and locally produced $P \mathrm{PE}_{2}$ and $P \mathrm{PG}_{2}$ are the main factors maintaining $\mathrm{DA}$ patency in utero [24]. The timing of closure of the DA after birth varies between species, but in humans it is usually completed within 48 hours [25]. In the full term infant, closure of the DA at birth occurs in two phases. The first phase, the 'functional' closure of the lumen, occurs within the first hours after birth as smooth muscle constricts. Several events promote DA constriction in the full term human newborn: 1) the increase in arterial $\mathrm{Po}_{2}, 2$ ) the decrease in blood pressure within the ductus lumen (due to the postnatal decrease in pulmonary vascular resistance), 3) the decrease in circulating $\mathrm{PGE}_{2}$ (due to the loss of placental PG production and the increase in PGs removal by the lung), as well as the decrease in the number of $\mathrm{PGE}_{2}$ receptors in the ductus wall [24, 25]. Constriction produces ischemic hypoxia of the vessel wall because the full-term human DA is a thick-walled muscular vessel that requires blood flowing both through its lumen and through the vasa vasorum to provide adequate oxygen delivery to its cells. DA constriction after birth diminishes blood flow from both of these sources, leading to profound hypoxia in the vessel wall [26]. Hypoxia inhibits local production of $\mathrm{PGE}_{2}$ and nitric oxide (NO) and induces production of growth factors. Thus, the first phase of functional closure leads to the second phase that involves 'anatomic' lumen occlusion and remodeling over the next several days due to extensive neointimal thickening and loss of smooth muscle cells from the inner muscle media $[24,25]$.

Very recently, Echtler et al. [27] proposed a model of closure of mice DA in which the initial constriction results in changes in the DA wall which adopts a prothrombotic phenotype with endothelial activation, deposition of von Willebrand factor and fibrin(ogen), and eventually endothelial cell detachment from the internal elastic lamina, leading to collagen exposure. This process triggers the accumulation of platelets circulating in the residual DA lumen and a platelet plug that seals the residual lumen is formed. In preterm human infants, like in mice, the DA is a much thinner vessel, able to extract all of the oxygen it needs from blood flow within its lumen. Therefore, and even when it does 
constrict, the premature DA frequently fails to develop profound hypoxia and neointimal expansion is markedly diminished [26]. The underdeveloped neointimal mounds fail to occlude the residual lumen and, as a result, the DA remains patent. Future research should address the question of whether platelet aggregation and thrombus formation can substitute for the lack of neointimal mounds in preterm human infants [26]. Echtler et al. [27] also demonstrated that thrombocytopenia was an independent predictor for failure of DA closure in preterm human newborns. However, their findings have not been confirmed by other investigators $[28,29]$.

\subsubsection{Oxygen and mammalian DA}

As mentioned above, the increase in $\mathrm{O}_{2}$ tension at birth is a key factor stimulating DA constriction which precedes the anatomical and permanent closure of the vessel [24-26]. The DA smooth muscle cell is the site of oxygensensing, whereas the endothelium releases vasoactive substances that are important in modulating DA tone [30, 31]. DA smooth muscle cells belong to those specialized cell types that sense local $\mathrm{O}_{2}$ tension, which include, among others, glomus cells of the carotid body, neuroepithelial cells in the lungs and smooth muscle cells of the resistance pulmonary arteries and of the fetoplacental arteries [30]. Interestingly, the pulmonary and the fetoplacental arteries contract in response to a decrease in $\mathrm{O}_{2}$ tension, whereas the DA contracts when $\mathrm{O}_{2}$ levels increase at birth. Decades of subsequent studies have failed to provide consensus on the mechanism for oxygen-induced DA constriction, possibly because multiple interacting pathways are involved [32]. The current two most accepted schemes, which are not mutually exclusive [32, 33], involve oxygen sensing/signaling 1) through redox state and ion channels and 2) through a cytochrome P450 (CYP450)/ET-1 complex. We will discuss these schemes in the following paragraphs.

\section{Redox state and ion channels}

Generation of reactive oxygen species (ROS), including superoxide anion and $\mathrm{H}_{2} \mathrm{O}_{2}$, may represent the earliest step in oxygen sensing by the DA. Mitochondria are critical for ROS generation, through the activity of specific mitochondrial enzymes (e.g., NADPH oxidase) and the electron transport chain (ETC). Membrane NADPH oxidase appears to be another source of ROS. In addition, activation of neutral sphingomyelinase, production of ceramide and subsequent NADPH oxidase-derived ROS might represent an amplification 
pathway downstream the mitochondrial sensor. The effects of ROS are mediated through redox-sensitive, voltage-gated potassium channels (Kv channels) [34, 35].

Inhibition of $\mathrm{Kv}$ channels results in membrane depolarization, and $\mathrm{Ca}^{2+}$ entry through voltage operated $\mathrm{Ca}^{2+}$ channels (VOCC) $[34,35]$. $\mathrm{Ca}^{2+}$ entry through store-operated channels [36-38] or its release from intracellular stores [39] may also contribute to the normoxic contraction of the DA [35]. ROS-mediated activation and induction of Rho kinase has been identified as another downstream effector of the ductal $\mathrm{O}_{2}$-sensing system in mammalian DA [36, 37, 40]. Rho/Rho-kinase pathways can induce calcium sensitization sustaining vasoconstriction as a result of persistent myosin light-chain phosphorylation [37, 40].

\section{CYP450/ET-1 complex}

In brief, it is postulated that a CYP450 of the $3 \mathrm{~A}$ subfamily, identified with CYP3A13 in the mouse, acts as the main oxygen sensor and links with ET-1, serving as the effector for the sustained contraction [33, 41]. A hitherto uncharacterized CYP3A-based monooxygenase product would serve as the messenger from the sensor to the effector. In addition, a direct action of oxygen on ET-1 production would reinforce ductal contraction [33, 41]. As pointed out above, the two pathways proposed for oxygen-induced contraction of the DA are not mutually exclusive since ET-1 can increase ROS production [42, 43], can interfere with $\mathrm{K}+$ channel function $[33,39,44]$ or can signal through Rho kinase inhibition [45]. Further investigation is required to determine whether links exist between these signaling systems to actively mediate oxygen-induced DA constriction.

\subsubsection{Prostaglandins and mammalian $D A$}

PGs are products of $P G$ endoperoxide $H$ synthase types 1 and 2, commonly referred to as cyclooxygenase-1 and cyclooxygenase-2 (COX-1 and COX-2). The COX product prostaglandin endoperoxide $\mathrm{H} 2$ is then converted by specific synthases to one of several different prostanoids, including $\mathrm{PGE}_{2}, \mathrm{PGD}_{2}, \mathrm{PGI}_{2}$, $\mathrm{PGF}_{2 \mathrm{a}}$, and $\mathrm{TXA}_{2}$ [46]. As mentioned above, considerable evidence supports a major role for $\mathrm{PGE}_{2}$ in maintaining mammalian fetal patency and for the rapid decline in $\mathrm{PGE}_{2}$ levels as trigger of DA closure after birth (see [24, 47] for reviews). In blood vessels, $\mathrm{PGE}_{2}$ acts on four different prostanoid receptors: $\mathrm{EP}_{1}, \mathrm{EP}_{2}, \mathrm{EP}_{3}$ and $\mathrm{EP}_{4}$. Both $\mathrm{EP}_{2}$ - and $\mathrm{EP}_{4}$-receptor subtypes mediate $\mathrm{PGE}_{2}$ relaxation through a cyclic AMP (cAMP) -dependent mechanism [48]. EP $\mathrm{P}_{1}$ is a 
constrictive receptor and $\mathrm{EP}_{3}$ induces a decline in cAMP, inhibiting smooth muscle relaxation [48]. The main receptor responsible for $\mathrm{PGE}_{2}$-induced DA relaxation varies among mammalian species and was reported as $\mathrm{EP}_{4}$ in rabbits [47, 49, 50], mice [51], rats [52], lambs [53], baboon [53], and humans [54], and as $\mathrm{EP}_{2}$ in pigs [55] and lambs [56]. The cAMP-inhibiting $\mathrm{EP}_{3}$ receptor is also present in the rabbit and piglet $D A$, modulating the dilating effect of $\mathrm{PGE}_{2}[47$, $49,50,55]$. In contrast, stimulation of $\mathrm{EP}_{3}$ receptor in the lamb DA produces not contraction but relaxation via a cAMP-independent pathway [56].

1.4.1.3 Other vasoactive pathways participating in the control of mammalian DA tone

Besides oxygen and PGs, the mammalian DA is sensitive to a wide range of vasoactive mediators [47, 57]. These include nitric oxide (NO), ET-1, catecholamines, carbon monoxide, vasoconstrictor prostanoids, plateletactivating factor (PAF), adenosine, or atrial natriuretic peptide (ANP), among others [47,57], The multiplicity of these contractile systems seems at odds with the relatively simple physiological role of the DA [47]. This can be explained by the fact that the two main systems that vary at birth, namely oxygen tension and $\mathrm{PGE}_{2}$, act synergistically to modulate the response of the $\mathrm{DA}$ to other vasoactive agents $[47,57]$.

\subsubsection{Chicken $D A$}

The chicken DA is the result of the fusion of two vessels with different embryological origin, morphology and functionality. The pulmonary side consists almost exclusively of neural crest derived cells, shows the structure of a muscular artery and responds to $\mathrm{O}_{2}$ with contraction, whereas the aortic part is of mesodermal origin, shows the morphology of an elastic artery and relaxes in response to $\mathrm{O}_{2}[18,22,38,58-60]$. Thus, the pulmonary side would be the "real" sixth aortic arch-derived DA, whereas the aortic side is the result of the incorporation of dorsal aorta tissue [22]. The two parts of the DA show marked quantitative differences in the response to vasoactive agents and, most strikingly, qualitative differences in the response to $\mathrm{O}_{2}$ (as shown in Table 1.1) $[38,59,61-63]$. 
Table 1.1. Effect of vasoactive mediators in the pulmonary (PulmDA) and the aortic (AoDA) side of chicken DA.

\begin{tabular}{|l|l|l|l|l|l|}
\hline $\begin{array}{l}\text { Vasoactive } \\
\text { agent }\end{array}$ & $\begin{array}{l}\text { Nature or mechanism of } \\
\text { action }\end{array}$ & $\begin{array}{l}\text { Effect in } \\
\text { PulmDA }\end{array}$ & $\begin{array}{l}\text { Effect in } \\
\text { AoDA }\end{array}$ & Ref. \\
\hline Oxygen & Gaseous mediator & Contraction & $\neq$ & Relaxation & $(59)$ \\
\hline $\mathrm{KCl}$ & Depolarizing agent & Contraction & $<$ & Contraction & $(59)$ \\
\hline PGE $_{2}$ & Prostaglandin & Contraction1 & $>$ & Contraction & $(62)$ \\
\hline Norepinephrine & Adrenoceptor agonist & Contraction & $>$ & Contraction & $(59)$ \\
\hline U46619 & TP receptor agonist & Contraction & $>$ & Contraction & $(59)$ \\
\hline Isoproterenol & B-adrenoceptor agonist & Relaxation & $>$ & Relaxation & $(62)$ \\
\hline Forskolin & Adenylyl cyclase activator & Relaxation & $>$ & Relaxation & $(62)$ \\
\hline Milrinone & $\begin{array}{l}\text { Phosphodiesterase-3 } \\
\text { inhibitor }\end{array}$ & Relaxation & $>$ & Relaxation & $(62)$ \\
\hline CO 2 & Muscarinic agonist & Relaxation 2 & $<$ & Relaxation & $(61)$ \\
\hline SNP & NO-donor & Relaxation & $<$ & Relaxation & $(61)$ \\
\hline sGC stimulator & Relaxation & $<$ & Relaxation & $(61)$ \\
\hline
\end{tabular}

${ }^{1}$ In the presence of TP receptor blockade, $\mathrm{PGE}_{2}$ evokes relaxation

${ }^{2}$ Concentrations $>3 \mu \mathrm{M}$ of acetylcholine evoke contraction

\subsubsection{Oxygen and chicken DA}

Closure of the chicken DA begins during the external pipping stage of hatching when the embryo begins to respire normoxic air with its lungs [18]. This DA constriction coincides with an increase in arterial blood oxygen levels [15], increased $\mathrm{O}_{2}$-induced contraction, and is complete by two days post-hatching [18]. Very recently, our group and other laboratory have characterized a mechanism implicating the mitochondrial electron transport chain as sensor, $\mathrm{H}_{2} \mathrm{O}_{2}$ as mediator, and $\mathrm{Kv}$ channels and Rho kinase as effectors of $\mathrm{O}_{2}$-induced 
contraction in the chicken DA ([38] and present Thesis), similar to that found in the mammalian DA. In addition, the postnatal increase in $\mathrm{O}_{2}$ is not only one the main trigger for constriction of the chicken DA but also has a profound modulatory effect on other vasoactive systems favoring the action of vasoconstrictors and decreasing the action of vasodilators [59, 61, 62].

\subsubsection{Prostaglandins and chicken $D A$}

In contrast to the DA of the lamb, mouse, rat, rabbit, or pig [47], the avian DA did not contract in response to cyclooxygenase (COX) inhibitors, suggesting that locally derived prostanoids do not exert a tonic vascular effect [38, 59, 64]. However, in a recent study we demonstrated a COX-mediated basal production of $P G E_{2}, P G F_{2 a}$. and $T_{X A_{2}}$ by the chicken DA, which indicates that COX is effectively active in this vessel [65]. Agren et al. [62] characterized the response of the chicken DA to $P E_{2}$ and found that in the 15-d chicken DA, $P G E_{2}$ elicited a relaxant response, whereas in 19-d DA it caused contraction. Interestingly, TP receptor occupancy either by an agonist (U46619) or an antagonist (SQ29,548) resulted in the appearance or the increase of the relaxant response to $\mathrm{PGE}_{2}$. This is consistent with a stimulatory action of $\mathrm{PGE}_{2}$ on TP receptors. Nevertheless, the relaxant potency and efficacy of $P E_{2}$ in the chicken DA, even after blocking its effect on the TP receptor, was very low. Very recently, Greyner \& Dzialowski [38] confirmed the contractile effects of $\mathrm{PGE}_{2}, \mathrm{PGD}_{2}$, and $\mathrm{PGF}_{2 a}$ in the chicken DA. Therefore, it appears that during embryonic development there is little tonic effect of local PGs on mediating relaxation of the chicken DA and that prostanoids are more likely to be vasoconstrictors.

\subsubsection{Endothelium-dependent relaxation and contraction in chicken $D A$}

Endothelial cells control the tone of the underlying vascular smooth muscle cells by releasing various relaxing and contracting factors [66]. The main vasoconstrictors are ET-1 and TXA 2 , while at least three endothelium derived relaxant factors (EDRF) have been found. These include NO, PGs (mainly prostacyclin, $\mathrm{PGI}_{2}$ ) and a yet unidentified factor called endothelium-derived hyperpolarizing factor (EDHF, [67-70]. Endothelium-dependent agonists such as acetylcholine and bradykinin, in addition to physical vascular stimuli such as shear stress, stimulate endothelial cells to release NO, $\mathrm{PGI}_{2}$ and EDHF [71]. The contribution of each of these factors to endothelium-dependent vasodilatation varies across vascular beds and also with the physiological or pharmacological stimuli used to stimulate the endothelium [68, 72]. 
The endothelium is also an important modulator of the vascular tone of the chicken DA during in ovo life and during its closure at hatching [61, 62]. Acetylcholine induces a concentration-dependent response in DA in fetal chickens. Low concentrations induce endothelium-dependent relaxation of the chicken DA mediated via NO and EDHF. High concentrations induce an endothelium-dependent contraction. Oxygen-induced contraction of the DA is also modulated by the endothelium: increases with inhibition of NO synthase or soluble guanylate cyclase and decreases in the presence of ET-1 receptor blockers [59].

Endothelial damage is common to numerous vascular diseases but, interestingly, occurs as a normal developmental process in the DA. When examined by scanning electron microscopy, the endothelium of the DA from the fetus prior to internal pipping shows a smooth and continuous surface [61]. In contrast, the intimal surface of DAs harvested from externally-pipped embryos has an irregular endothelial lining with protrusion and detachment of endothelial cells, leaving large areas of exposed subendothelial tissue [61]. This process of endothelial detachment has been confirmed by other investigators [18] and is accompanied by a marked impairment in NO production and endotheliummediated relaxation.

As mentioned above, the predominant action of acetylcholine (ACh) in most vascular beds is endothelium-dependent relaxation [66, 73]. However, it is known that in some blood vessels, and under certain conditions, ACh may induce a contractile response through the release of one or more EDCFs [74-76] or by direct stimulation of muscarinic receptors in smooth muscle cells $[77,78]$. ACh-induced vasoconstricition varies among species and vascular beds and frequently depends on the vascular tone. Thus under resting tone conditions ACh induces contraction, whereas when the vessels are precontracted with an agonist ACh induces relaxation [79-81]. Schuurman and Villamor [65] demonstrated that $\mathrm{ACh}$, at a concentration $>1 \mu \mathrm{M}$, induced an endothelium-dependent contraction of chicken DA through stimulation of muscarinic receptors. This contraction is mediated by a COX-1 metabolite and involves stimulation of TP receptors. Therefore, ACh evokes endotheliumdependent contraction and relaxation in the chicken DA. 
1.4.2.4 Other vasoactive pathways participating in the control of chicken DA tone

Besides oxygen, PGs and Ach, the chicken DA is also responsive to catecholamines [59], NO [61], ET-1 [61], adenylate cyclase activation [62] and phosphodiesterase [62].

For some of these vasoactive mediators, it was observed a marked difference in responsiveness between the pulmonary and the aortic side of the DA (Table 1.1), suggesting the presence of certain selectivity for the DA. Also developmental changes have been observed for the majority of these vasoactive mediators (see below).

\subsubsection{Developmental changes in chicken DA reactivity}

To ensure proper constriction after birth, the mammalian DA acquires vasoactive competence early in development and changes in responsiveness with advancing gestational age [22, 24, 47]. Similarly, the chicken DA undergoes a developmental increase in its contractile responsiveness [59, 64] and a developmental decrease in its responsiveness to several relaxant agonists [61, 62] (Table 1.2).

In the chicken DA a significant contraction to high-potassium depolarizing solution is present in the 15-day embryo (0.7 incubation), indicating that at this developmental stage DA smooth muscle cells are already equipped with contractile proteins and a mature excitation-contraction coupling [59]. The relative small increase in the response to depolarization between 15 and 19 days (2-fold), compared to the increase in carotid and femoral arteries (5-7-fold) [82], implies an early maturation of the smooth muscle in the DA. In contrast, the 15-day DA did not respond to $\mathrm{O}_{2}$ or a-adrenoceptor agonists, but the presence of responses to the thromboxane $A_{2}$ mimetic U46619 and ET-1 indicates that pharmacomechanical coupling is also developed rather early in this vessel [59]. 
Table 1.2. Developmental changes in the response of chicken pulmDA to vasoactive mediators

\begin{tabular}{|c|c|c|c|c|c|c|c|}
\hline $\begin{array}{l}\text { Vasoactive } \\
\text { agent }\end{array}$ & $\begin{array}{l}\text { Nature/mechanism } \\
\text { of action }\end{array}$ & 15-d DA & & 19-d DA & & 21-d DA & Ref \\
\hline Oxygen & Gaseous mediator & No response & $\neq$ & Contraction & $<$ & Contraction & (59) \\
\hline $\mathrm{KCl}$ & Depolarizing agent & Contraction & $k$ & Contraction & k & Contraction & (59) \\
\hline 4-AP & $\mathrm{K}_{\mathrm{V}}$-channel blocker & Contraction & $k$ & Contraction & $k$ & Contraction & (59) \\
\hline Norepinephrine & Adrenoceptor agonist & No response & $\neq$ & Contraction & k & Contraction & (59) \\
\hline Phenylephrine & $\begin{array}{l}\text { a-adrenoceptor } \\
\text { agonist }\end{array}$ & No response & $\neq$ & Contraction & $<$ & Contraction & (59) \\
\hline U46619 & TP receptor agonist & Contraction & $k$ & Contraction & $=$ & Contraction & (59) \\
\hline Endothelin-1 & Polipeptide & Contraction & $k$ & Contraction & $>$ & Contraction & (59) \\
\hline ACh $(\geq 3 \mu \mathrm{M})$ & Muscarinic agonist & Contraction & $k$ & Contraction & $>$ & Contraction & (65) \\
\hline $\mathrm{PGE}_{2}$ & Prostaglandin & Relaxation & $\neq$ & Contraction & $\neq$ & No response & (62) \\
\hline $\begin{array}{l}\mathrm{PGE}_{2} \\
\text { (+TP-blockade) }\end{array}$ & Prostaglandin & Relaxation & $p$ & Relaxation & $p$ & Relaxation & (62) \\
\hline $\mathrm{ACh}(<3 \mu \mathrm{M})$ & Muscarinic agonist & Relaxation & $>$ & Relaxation & $p$ & Relaxation & (61) \\
\hline SNP & NO-donor & Relaxation & $=$ & Relaxation & $p$ & Relaxation & (61) \\
\hline Isoproterenol & $\begin{array}{l}\beta \text {-adrenoceptor } \\
\text { agonist }\end{array}$ & Relaxation & $=$ & Relaxation & $p$ & Relaxation & (62) \\
\hline BAY 41-2272 & sGC stimulator & Relaxation & $=$ & Relaxation & $=$ & Relaxation & (61) \\
\hline 8-Br cGMP & cGMP analog & Relaxation & $=$ & Relaxation & $=$ & Relaxation & (61) \\
\hline Forskolin & $\begin{array}{l}\text { Adenylyl cyclase } \\
\text { activator }\end{array}$ & Relaxation & $=$ & Relaxation & $=$ & Relaxation & (62) \\
\hline Milrinone & $\begin{array}{l}\text { Phosphodiesterase-3 } \\
\text { inhibitor }\end{array}$ & Relaxation & $<$ & Relaxation & $=$ & Relaxation & (62) \\
\hline
\end{tabular}


As mentioned above, the immature (15-day) chicken DA was unresponsive to oxygen and a-adrenoceptor agonists [59]. Interestingly, the time course of catecholamines in the plasma of chicken embryos is characterized by a maximum of epinephrine and NE at day 19, shortly before lung ventilation (i.e. internal pipping) is initiated [83, 84]. This process of internal pipping is also accompanied by an increase in $\mathrm{O}_{2}$ concentration. Agren et al. [59] observed that the efficacy (but not the potency) of NE increased when 19-day DA were bubbled with high $\mathrm{O}_{2}$ concentrations. In addition, when 21-day DA was precontracted with $\mathrm{NE}$, the $\mathrm{O}_{2}$-evoked response was larger than under basal conditions. It can be speculated that the presence of higher $\mathrm{O}_{2}$ tension, higher catecholamines concentrations, the positive interactions between $\mathrm{O}_{2-}$ and $\mathrm{NE}$ induced contractions, and a developmental increase in the sensitivity of DA to these vasoactive mediators are critical factors involved in the closure of chicken DA during transition to ex ovo life [59]. 


\section{References}

1. Tchirikov, M., H.J. Schroder, and K. Hecher, Ductus venosus shunting in the fetal venous circulation: regulatory mechanisms, diagnostic methods and medical importance. Ultrasound Obstet Gynecol, 2006. 27(4): p. 452-61.

2. Amoroso, E.C., et al., The bifurcation of the posterior caval channel in the eutherian foetal heart. J Anat, 1942. 76(Pt 3): p. 240-7.

3. White, P.T., Experimental studies on the circulatory system of the late chick embryo. J Exp Biol, 1974. 61(3): p. 571-92.

4. Romanoff, A.L., The avian embryo. Structural and functional development. 1960, New York: The Macmillan Company.

5. Rasanen, J., et al., Role of the pulmonary circulation in the distribution of human fetal cardiac output during the second half of pregnancy. Circulation, 1996. 94(5): p. 1068-73.

6. Anderson, D.F., et al., Central shunt flows and pressures in the mature fetal lamb. Am J Physiol, 1981. 241(1): p. H60-6.

7. Mielke, G. and N. Benda, Cardiac output and central distribution of blood flow in the human fetus. Circulation, 2001. 103(12): p. 1662-8.

8. Rudolph, A.M., Fetal and neonatal pulmonary circulation. Am Rev Respir Dis, 1977. 115(6 Pt 2): p. 11-8.

9. Anderson, D.F., et al., Flow through the foramen ovale of the fetal and new-born lamb. J Physiol, 1985. 365: p. 29-40.

10. Murphy, P.J., The fetal circulation. Continuing Education in Anaesthesia, Critical Care \& Pain, 2005. 5(4): p. 107-112.

11. Kiserud, T., et al., Foramen ovale: an ultrasonographic study of its relation to the inferior vena cava, ductus venosus and hepatic veins. Ultrasound Obstet Gynecol, 1992. 2(6): p. 389-96.

12. Kiserud, T., et al., Effect of NO, phenylephrine, and hypoxemia on ductus venosus diameter in fetal sheep. Am J Physiol Heart Circ Physiol, 2000. 279(3): p. H1166-71.

13. Kiserud, T., S. Rasmussen, and S. Skulstad, Blood flow and the degree of shunting through the ductus venosus in the human fetus. Am J Obstet Gynecol, 2000. 182(1 Pt 1): p. 147-53.

14. Tchirikov, M., M. Strohner, and A. Scholz, Cardiac output and blood flow volume redistribution during acute maternal hypoxia in fetal sheep. J Perinat Med, 2010. 38(4): p. 387-92.

15. Takenaka, T.a., Cardiovascular shunt and model analysis in the chick embryo. Cardiovascular shunts. 1985, Copenhagen: Munksgaard. 179-194.

16. H. Rahn, S.M., P.R. Sotherland, Circulatory changes and oxygen delivery in the chick embryo prior to hatching, in Cardiovascular shunts, W.W.B. K. Johansen, Editor. 1985, Munksgaard: Copenhagen. p. 199-211. 
17. Sbong, S. and E.M. Dzialowski, Respiratory and cardiovascular responses to acute hypoxia and hyperoxia in internally pipped chicken embryos. Comp Biochem Physiol A Mol Integr Physiol, 2007. 148(4): p. 761-8.

18. Belanger, C., et al., Morphological changes in the chicken ductus arteriosi during closure at hatching. Anat Rec (Hoboken), 2008. 291(8): p. 1007-15.

19. Tazawa, H., et al., Gas exchange, blood gases and acid-base status in the chick before, during and after hatching. Respir Physiol, 1983. 53(2): p. 173-85.

20. Mulder, A.L., et al., Cardiac output distribution in response to hypoxia in the chick embryo in the second half of the incubation time. J Physiol, 1998. 508 ( Pt 1): p. 281-7.

21. Mulder, A.L., et al., Sympathetic control of the cardiovascular response to acute hypoxemia in the chick embryo. Am J Physiol Regul Integr Comp Physiol, 2002. 282(4): p. R1156-63.

22. Bergwerff, M., M.C. DeRuiter, and A.C. Gittenberger-de Groot, Comparative anatomy and ontogeny of the ductus arteriosus, a vascular outsider. Anat Embryol (Berl), 1999. 200(6): p. 559-71.

23. Reese, J., Death, dying, and exhaustion in the ductus arteriosus: prerequisites for permanent closure. Am J Physiol Regul Integr Comp Physiol, 2006. 290(2): p. R357-8.

24. Clyman, R.I., Mechanisms regulating the ductus arteriosus. Biology of the Neonate, 2006. 89(4): p. 330-335.

25. Clyman, R.I., Mechanisms regulating closure of the ductus arteriosus, in Fetal and Neonatal Physiology, R.A. Polin, W.W. Fox, and S.H. Abman, Editors. 2004, Saunders: Philadelphia. p. 743-748.

26. Clyman, R. and S. Chemtob, Vessel remodeling in the newborn: platelets fill the gap. Nat Med. 16(1): p. 33-5.

27. Echtler, K., et al., Platelets contribute to postnatal occlusion of the ductus arteriosus. Nat Med, 2010. 16(1): p. 75-82.

28. Fujioka, K., et al., Does thrombocytopenia contribute to patent ductus arteriosus? Nat Med, 2011. 17(1): p. 29-30; author reply 30-1.

29. Shah, N.A., et al., Relationship between circulating platelet counts and ductus arteriosus patency after indomethacin treatment. J Pediatr, 2011. 158(6): p. 919-923 e1-2.

30. Weir, E.K., et al., Acute oxygen-sensing mechanisms. N Engl J Med, 2005. 353(19): p. 2042-55.

31. Sutendra, G. and E.D. Michelakis, The chicken embryo as a model for ductus arteriosus developmental biology: cracking into new territory. Am J Physiol Regul Integr Comp Physiol, 2007. 292(1): p. R481-4.

32. Stoller, J.Z., et al., Current Perspectives on Pathobiology of the Ductus Arteriosus. J Clin Exp Cardiolog, 2012. S8:001.

33. Coceani, F. and B. Baragatti, Mechanisms for ductus arteriosus closure. Semin Perinatol, 2012. 36(2): p. 92-7. 
34. Michelakis, E., et al., Voltage-gated potassium channels in human ductus arteriosus. Lancet, 2000. 356(9224): p. 134-7.

35. Weir, E.K., et al., Redox signaling in oxygen sensing by vessels. Respir Physiol Neurobiol, 2002. 132(1): p. 121-30.

36. Hong, Z., et al., Role of store-operated calcium channels and calcium sensitization in normoxic contraction of the ductus arteriosus. Circulation, 2006. 114(13): p. 1372-9.

37. Clyman, R.I., et al., Calcium-dependent and calcium-sensitizing pathways in the mature and immature ductus arteriosus. Am J Physiol Regul Integr Comp Physiol, 2007. 293(4): p. R1650-6.

38. Greyner, H. and E.M. Dzialowski, Mechanisms mediating the oxygen-induced vasoreactivity of the ductus arteriosus in the chicken embryo. Am J Physiol Regul Integr Comp Physiol, 2008. 295(5): p. R1647-59.

39. Keck, M., et al., Oxygen increases ductus arteriosus smooth muscle cytosolic calcium via release of calcium from inositol triphosphate-sensitive stores. Am J Physiol Lung Cell Mol Physiol, 2005. 288(5): p. L917-23.

40. Kajimoto, H., et al., Oxygen activates the Rho/Rho-kinase pathway and induces RhoB and ROCK-1 expression in human and rabbit ductus arteriosus by increasing mitochondria-derived reactive oxygen species: a newly recognized mechanism for sustaining ductal constriction. Circulation, 2007. 115(13): $p$. 1777-88.

41. Baragatti, B., et al., Cytochrome P-450 3A13 and endothelin jointly mediate ductus arteriosus constriction to oxygen in mice. Am J Physiol Heart Circ Physiol, 2011. 300(3): p. H892-901.

42. Li, L., et al., Endothelin-1 increases vascular superoxide via endothelin(A)NADPH oxidase pathway in low-renin hypertension. Circulation, 2003. 107(7): p. 1053-8.

43. Perez-Vizcaino, F., A. Cogolludo, and L. Moreno, Reactive oxygen species signaling in pulmonary vascular smooth muscle. Respir Physiol Neurobiol, 2010. 174(3): p. 212-20.

44. Coceani, F., et al., Endothelin A receptor is necessary for $\mathrm{O}(2)$ constriction but not closure of ductus arteriosus. Am J Physiol, 1999. 277(4 Pt 2): p. H1521-31.

45. Budzyn, K., P.D. Marley, and C.G. Sobey, Targeting Rho and Rho-kinase in the treatment of cardiovascular disease. Trends Pharmacol Sci, 2006. 27(2): p. 97-104.

46. Belik, J., et al., Isoprostanes in fetal and neonatal health and disease. Free Radic Biol Med, 2010. 48(2): p. 177-88.

47. Smith, G.C., The pharmacology of the ductus arteriosus. Pharmacol Rev, 1998. 50(1): p. 35-58.

48. Narumiya, S. and G.A. FitzGerald, Genetic and pharmacological analysis of prostanoid receptor function. J Clin Invest, 2001. 108(1): p. 25-30. 
49. Smith, G.C., R.A. Coleman, and J.C. McGrath, Characterization of dilator prostanoid receptors in the fetal rabbit ductus arteriosus. J Pharmacol Exp Ther, 1994. 271(1): p. 390-6.

50. Smith, G.C. and J.C. McGrath, Contractile effects of prostanoids on fetal rabbit ductus arteriosus. J Cardiovasc Pharmacol, 1995. 25(1): p. 113-8.

51. Nguyen, M., et al., The prostaglandin receptor EP4 triggers remodelling of the cardiovascular system at birth. Nature, 1997. 390(6655): p. 78-81.

52. Kajino, H., et al., An EP4 receptor agonist prevents indomethacin-induced closure of rat ductus arteriosus in vivo. Pediatr Res, 2004. 56(4): p. 586-90.

53. Waleh, N., et al., Prostaglandin E2--mediated relaxation of the ductus arteriosus: effects of gestational age on $g$ protein-coupled receptor expression, signaling, and vasomotor control. Circulation, 2004. 110(16): p. 2326-32.

54. Leonhardt, A., et al., Expression of prostanoid receptors in human ductus arteriosus. Br J Pharmacol, 2003. 138(4): p. 655-9.

55. Bhattacharya, M., et al., Developmental changes in prostaglandin $\mathrm{E}(2)$ receptor subtypes in porcine ductus arteriosus. Possible contribution in altered responsiveness to prostaglandin E(2). Circulation, 1999. 100(16): p. 1751-6.

56. Bouayad, A., et al., Characterization of PGE2 receptors in fetal and newborn lamb ductus arteriosus. Am J Physiol Heart Circ Physiol, 2001. 280(5): p. H2342-9.

57. Reese, J., et al., Inadvertent relaxation of the ductus arteriosus by pharmacologic agents that are commonly used in the neonatal period. Semin Perinatol, 2010. 34(3): p. 222-30.

58. Bergwerff, M., et al., Neural crest cell contribution to the developing circulatory system: implications for vascular morphology? Circ Res, 1998. 82(2): p. 221-31.

59. Agren, P., et al., Ontogeny of chicken ductus arteriosus response to oxygen and vasoconstrictors. Am J Physiol Regul Integr Comp Physiol, 2007. 292(1): p. R485-96.

60. Copeland, J. and E.M. Dzialowski, Effects of hypoxic and hyperoxic incubation on the reactivity of the chicken embryo (Gallus gallus) ductus arteriosi in response to catecholamines and oxygen. Exp Physiol, 2009. 94(1): p. 152-61.

61. Agren, P., et al., Developmental changes in endothelium-dependent relaxation of the chicken ductus arteriosus. J Physiol Pharmacol, 2008. 59(1): p. 55-76.

62. Agren, P., et al., Developmental changes in the effects of prostaglandin E2 in the chicken ductus arteriosus. J Comp Physiol B, 2009. 179(2): p. 133-43.

63. Moonen, R.M., et al., Response of chicken ductus arteriosus to hypercarbic and normocarbic acidosis. Neonatology, 2010. 98(1): p. 47-56.

64. Dzialowski, E.M. and H. Greyner, Maturation of the contractile response of the Emu ductus arteriosus. J Comp Physiol [B], 2008. 178(3): p. 401-12.

65. Schuurman, M.J. and E. Villamor, Endothelium-dependent contraction induced by acetylcholine in the chicken ductus arteriosus involves cyclooxygenase-1 activation and TP receptor stimulation. Comp Biochem Physiol A Mol Integr Physiol, 2010. 157(1): p. 28-34. 
66. Furchgott, R.F. and P.M. Vanhoutte, Endothelium-derived relaxing and contracting factors. Faseb J, 1989. 3(9): p. 2007-18.

67. Takizawa, T., E. Horikoshi, and A. Kamata, Role of the nitric oxide-cGMP system in the regulation of ductus arteriosus patency in fetal rats. J Vet Med Sci, 1999. 61(12): p. 1277-80.

68. Tare, M., H.C. Parkington, and H.A. Coleman, EDHF, NO and a prostanoid: hyperpolarization-dependent and -independent relaxation in guinea-pig arteries. Br J Pharmacol, 2000. 130(3): p. 605-18.

69. Kamper, A.M., L.C. Paul, and G.J. Blauw, Prostaglandins are involved in acetylcholine- and 5-hydroxytryptamine-induced, nitric oxide-mediated vasodilatation in human forearm. J Cardiovasc Pharmacol, 2002. 40(6): p. 922-9.

70. Sendao Oliveira, A.P. and L.M. Bendhack, Relaxation induced by acetylcholine involves endothelium-derived hyperpolarizing factor in 2-kidney 1-clip hypertensive rat carotid arteries. Pharmacology, 2004. 72(4): p. 231-9.

71. Scotland, R.S., et al., Investigation of vascular responses in endothelial nitric oxide synthase/cyclooxygenase-1 double-knockout mice: key role for endothelium-derived hyperpolarizing factor in the regulation of blood pressure in vivo. Circulation, 2005. 111(6): p. 796-803.

72. Arnal, J.F., et al., Endothelium-derived nitric oxide and vascular physiology and pathology. Cell Mol Life Sci, 1999. 55(8-9): p. 1078-87.

73. Rubanyi, G.M., Endothelium-derived relaxing and contracting factors. J Cell Biochem, 1991. 46(1): p. 27-36.

74. Katusic, Z.S., J.T. Shepherd, and P.M. Vanhoutte, Endothelium-dependent contractions to calcium ionophore A23187, arachidonic acid, and acetylcholine in canine basilar arteries. Stroke, 1988. 19(4): p. 476-9.

75. Usui, H., et al., Endothelium-dependent contraction produced by acetylcholine and relaxation produced by histamine in monkey basilar arteries. Life Sci, 1993. 52(4): p. 377-87.

76. Feletou, M., T.J. Verbeuren, and P.M. Vanhoutte, Endothelium-dependent contractions in SHR: a tale of prostanoid TP and IP receptors. Br J Pharmacol, 2009. 156(4): p. 563-74.

77. Ren, L.M., T. Nakane, and S. Chiba, Muscarinic receptor subtypes mediating vasodilation and vasoconstriction in isolated, perfused simian coronary arteries. J Cardiovasc Pharmacol, 1993. 22(6): p. 841-6.

78. Pesic, S., L. Grbovic, and A. Jovanovic, Acetylcholine-induced contractions in the perforating branch of the human internal mammary artery: protective role of the vascular endothelium. Pharmacology, 2002. 64(4): p. 182-8.

79. Miller, V.M. and P.M. Vanhoutte, Endothelium-dependent responses in isolated blood vessels of lower vertebrates. Blood Vessels, 1986. 23(4-5): p. 225-35.

80. Hyman, A.L. and P.J. Kadowitz, Tone-dependent responses to acetylcholine in the feline pulmonary vascular bed. J Appl Physiol, 1988. 64(5): p. 2002-9. 
81. Hyman, A.L. and P.J. Kadowitz, Influence of tone on responses to acetylcholine in the rabbit pulmonary vascular bed. J Appl Physiol, 1989. 67(4): p. 1388-94.

82. le Noble, F.A., et al., Contractile and relaxing reactivity in carotid and femoral arteries of chicken embryos. Am J Physiol Heart Circ Physiol, 2000. 278(4): p. H1261-8.

83. Wittmann, J. and J. Prechtl, Respiratory function of catecholamines during the late period of avian development. Respir Physiol, 1991. 83(3): p. 375-86.

84. Mulder, A.L., et al., Developmental changes in plasma catecholamine concentrations during normoxia and acute hypoxia in the chick embryo. $J$ Physiol, 2000. 527 Pt 3: p. 593-9. 


\title{
Chapter 2
}

\section{Maturation of $\mathrm{O}_{2}$ sensing and signaling in the chicken ductus arteriosus}

\author{
Angel L. Cogolludo, Javier Moral-Sanz, Saskia van der \\ Sterren, Giovanna Frazziano, Anne N.H. van Cleef, \\ Carmen Menéndez, Bea Zoer, Enrique Moreno, Angela Roman, \\ Francisco Pérez Vizcaino, Eduardo Villamor \\ Am J Physiol Lung Cell Mol Physiol 297: L619-L630, 2009
}




\begin{abstract}
The increase in $\mathrm{O}_{2}$ tension after birth is a major factor stimulating ductus arteriosus (DA) constriction and closure. Here we studied the role of the mitochondrial electron transport chain (ETC) as sensor, $\mathrm{H}_{2} \mathrm{O}_{2}$ as mediator, and voltage-gated potassium $(\mathrm{Kv})$ channels and Rho kinase as effectors of $\mathrm{O}_{2}$ induced contraction in the chicken DA during fetal development. Switching from $0 \%$ to $21 \% \mathrm{O}_{2}$ contracted the pulmonary side of the mature DA (mature pDA) but had no effect in immature pDA and relaxed the aortic side of the mature DA (mature aDA). This contraction of the pDA was attenuated by inhibitors of the mitochondrial ETC and by the $\mathrm{H}_{2} \mathrm{O}_{2}$ scavenger polyethylene glycol (PEG)catalase. Moreover, $\mathrm{O}_{2}$ increased reactive oxygen species (ROS) production, measured with the fluorescent probes dihydroethidium and 2',7'-dichlorofluorescein, only in mature pDA. The $\mathrm{H}_{2} \mathrm{O}_{2}$ analog $t$-butyl-hydroperoxide mimicked the responses to $\mathrm{O}_{2}$ in the three vessels. In contrast to immature pDA cells, mature pDA cells exhibited high-amplitude $\mathrm{O}_{2}$-sensitive potassium currents. The $\mathrm{K}_{\mathrm{v}}$ channel blocker 4-aminopyridine prevented the current inhibition elicited by $\mathrm{O}_{2}$. The L-type $\mathrm{Ca}^{2}$ ( $\mathrm{Ca}_{\mathrm{L}}$ ) channel blocker nifedipine and the Rho kinase inhibitors Y-27632 and hydroxyfasudil induced a similar relaxation when mature pDA were stimulated with $\mathrm{O}_{2}$ or $\mathrm{H}_{2} \mathrm{O}_{2}$. Moreover, the sensitivity to these drugs increased with maturation. Our results indicate the presence of a common mechanism for $\mathrm{O}_{2}$ sensing/signaling in mammalian and nonmammalian DA and favor the idea that, rather than a single mechanism, a parallel maturation of the sensor and effectors is critical for $\mathrm{O}_{2}$ sensitivity appearance during development.
\end{abstract}




\subsection{Introduction}

The ductus arteriosus (DA) is an artery that connects the main pulmonary artery with the aorta during fetal life. DA smooth muscle cells (DASMC) belong to those specialized cell types that sense local $\mathrm{O}_{2}$ tension, which include, among others, glomus cells of the carotid body, neuroepithelial cells in the lungs, and smooth muscle cells of the resistance pulmonary arteries and of the fetoplacental arteries $(4,38)$. In term infants, the increase in $\mathrm{O}_{2}$ tension at birth is a key factor stimulating DA constriction, which precedes the anatomic and permanent closure of the vessel $(9,29)$. In contrast, the DA from preterm babies is frequently unresponsive to $\mathrm{O}_{2}$ and less likely to constrict after birth. Failure of DA closure in very preterm infants is associated with several comorbidities, such as necrotizing enterocolitis, intracranial hemorrhage, pulmonary edema/ hemorrhage, bronchopulmonary dysplasia, and retinopathy (9). Thus a better understanding of the mechanisms involved in the maturation of the DA response to $\mathrm{O}_{2}$ may have direct clinical application.

Although the response to a change in $\mathrm{O}_{2}$ is diametrically opposite, the mechanisms responsible for smooth muscle contraction in hypoxic pulmonary vasoconstriction and normoxic contraction of the DA appear to be virtually the same $(25,38)$. In the $\mathrm{DA}, \mathrm{O}_{2}$-induced constriction is thought to be mediated by the inhibition of voltage-gated potassium $\left(\mathrm{K}_{\mathrm{v}}\right)$ channels, resulting in membrane depolarization, and $\mathrm{Ca}^{2}$ entry through voltage-operated $\mathrm{Ca}^{2}$ channels $(22,23$, 34). Michelakis et al. (23) proposed a model in which a rise in $\mathrm{O}_{2}$ modulates the function of the mitochondrial electron transport chain (ETC, the sensor), leading to an increased production of $\mathrm{H}_{2} \mathrm{O}_{2}$ (the mediator) that causes the inhibition of $K_{v}$ channels (the effector). Very recently, Rho kinase was identified as another downstream effector of the $\mathrm{O}_{2}$-sensing system in the DA $(11,17,18)$.

In recent years, the chicken embryo/fetus has emerged as an excellent model for the study of DA vascular biology (1, 16, 31). Our group and another laboratory have characterized the developmental changes in the responsiveness of the chicken $\mathrm{DA}$ to $\mathrm{O}_{2}$ as well as to other vasoactive agonists including prostaglandins, nitric oxide (NO), and catecholamines. We found (1) that responsiveness of chicken $\mathrm{DA}$ to $\mathrm{O}_{2}$ was developmentally regulated. It was very weak or absent at day 15 but present at day 19 of incubation (term is 21 days). A peculiar feature of the chicken DA is the presence of morphological and functional heterogeneity along its path between the pulmonary artery and the aorta $(1,6,7)$. Thus the pulmonary side shows the structure of a muscular artery and responds to $\mathrm{O}_{2}$ with contraction, i.e., similar to the mammalian DA, whereas the aortic part shows the morphology of an elastic artery and relaxes in 
response to $\mathrm{O}_{2}(1,7,16)$. Therefore, by comparison of the portion of the vessel that responds to $\mathrm{O}_{2}$ with contraction (i.e., the pulmonary side of the 19- to 20day $D A$ ) with the portion that responds with relaxation (i.e., the aortic side of the 19- to 20-day DA) and the portion unresponsive to $\mathrm{O}_{2}$ (i.e., the pulmonary side of the 15-day DA), the chicken DA offers a unique model to obtain information about the maturation of the mechanisms involved in $\mathrm{O}_{2}$ responsiveness. In the present study, we aimed to investigate the involvement of the mitochondrial ETC as sensor, $\mathrm{H}_{2} \mathrm{O}_{2}$ as mediator, and $\mathrm{K}_{\mathrm{V}}$ channels and Rho kinase as effectors of $\mathrm{O}_{2}$-induced contraction in the DA of developing fetal chickens.

\subsection{Methods}

Experiments were performed in accordance with the Spanish and Dutch laws for animal experimentation, and the procedures were approved by our institutional review boards.

\subsubsection{Egg incubation and vessel isolation}

Fertilized eggs of White Leghorn chickens were incubated at $37.8^{\circ} \mathrm{C}$ and $45 \%$ humidity and rotated once per hour (incubator model 25HS, Masalles Comercial). Embryos were incubated for 15 or 19-20 days of the 21-day incubation period. For clarity, we refer to immature DA (15 days) or mature DA (19-20 days) based on their ability to respond to acute changes in $\mathrm{O}_{2}$. On the experimental day, the fetuses were killed by decapitation and both the right and the left DA were carefully dissected and severed distal to the takeoff of the pulmonary arteries and proximal to the insertion into the aorta. Each DA was divided in two segments referred to as pulmonary and aortic DA (pDA and aDA, respectively). The boundary between the $\mathrm{pDA}$ and the aDA was determined on the basis of the marked differences of diameter observed along the vessel (1, 35). The junction between the two segments was discarded for experiments.

\subsubsection{Recording of arterial reactivity}

Two stainless steel wires (diameter $40 \mu \mathrm{m}$ ) were inserted into the lumen of the DA, which was mounted as a ring segment between an isometric force transducer and a displacement device in a myograph (Danish Myo Technology model 610M, Aarhus, Denmark). The myograph organ bath (5-ml vol) was filled with Krebs-Ringer bicarbonate buffer (in mmol/l: $118.5 \mathrm{NaCl}, 4.75 \mathrm{KCl}, 1.2$ $\mathrm{MgSO}_{4} \cdot 7 \mathrm{H}_{2} \mathrm{O}, 1.2 \mathrm{KH}_{2} \mathrm{PO}_{4}, 25 \mathrm{NaHCO}_{3}, 2.5 \mathrm{CaCl}_{2}$, and 5.5 glucose) 
maintained at $39^{\circ} \mathrm{C}$ and continuously aerated with $0 \% \mathrm{O}_{2}-95 \% \mathrm{~N}_{2}-5 \% \mathrm{CO}_{2}$ $\left(\mathrm{PO}_{2}\right.$ 2.6-3.3 kPa). Each DA was stretched to its individual optimal lumen diameter, i.e., the diameter at which it developed the strongest contractile response to $\mathrm{KCl}\left(62.5 \times 10^{-3} \mathrm{M}\right)$, with a diameter-tension protocol as previously described $(1,36)$. The response of the DA rings to oxygen was assessed by bubbling the organ chamber with $21 \% \mathrm{O}_{2}-74 \% \mathrm{~N}_{2}-5 \% \mathrm{CO}_{2}\left(\mathrm{PO}_{2} 17-19 \mathrm{kPa}\right)$.

\subsubsection{Determination of reactive oxygen species}

Reactive oxygen species (ROS) generation in isolated DA was assessed with 2',7'-dichlorofluorescein (DCF) or dihydroethidium (DHE), which detect mainly $\mathrm{H}_{2} \mathrm{O}_{2}$ and superoxide, respectively. Endothelium-denuded DA were incubated with the membrane-permeant diacetate form of DCF (DCFDA, 10-5 M) or with DHE $\left(10^{-5} \mathrm{M}\right)$ for $60 \mathrm{~min}$. DA were then placed in the stage of a fluorescent inverted microscope (Leica DM IRB, Wetzlar, Germany) and superfused at $2 \mathrm{ml} /$ min with a physiological salt solution (PSS) of the following composition (in $\mathrm{mmol} / \mathrm{l}): 130 \mathrm{NaCl}, 5 \mathrm{KCl}, 1.2 \mathrm{MgCl}_{2}, 1.8 \mathrm{CaCl}_{2}, 10$ glucose, and $10 \mathrm{HEPES}(\mathrm{pH}$ 7.3 with $\mathrm{NaOH}$ ). During the first $30 \mathrm{~min}$ the tissues were maintained under hypoxia, induced by bubbling the PSS solution with $100 \% \mathrm{~N}_{2}$ to achieve a $\mathrm{PO}_{2}$ of $\approx 3-4 \mathrm{kPa}$ in the chamber. Once fluorescence values were stable, preparations were challenged with normoxic solution for $5 \mathrm{~min}$. DA were illuminated through the luminal surface with a $450-490 \mathrm{~nm}$ (for DCF) or 530-nm (for DHE) band-pass filter. The emitted fluorescence was filtered with 515-nm (for DCF) or 610-nm (for DHE) long-pass emission filters. Images were taken at 1-min intervals with a Leica DC300F color digital camera. Fluorescence, after subtraction of background, was quantified with ImageJ (version 1.32j, National Institutes of Health; http://rsb.info.nih/ij/). Intensity values are reported as a percentage of the values before the normoxic challenge.

\subsubsection{Electrophysiological studies}

DASMC were isolated by enzymatic digestion. Briefly, DA rings were opened along their longitudinal axis and placed into a nominally calcium-free PSS ( $\mathrm{Ca}^{2+-}$ free PSS). To establish a hypoxic environment during the DASMC isolation procedure, the $\mathrm{O}_{2}$ scavenger sodium dithionite $\left(0.8 \times 10^{-3} \mathrm{M}\right.$; $\mathrm{pH}$ adjusted to 7.4 with $\mathrm{NaOH}$ ) was included in the $\mathrm{Ca}^{2+-}$-free PSS as reported previously (26). Tissues were initially incubated at $4^{\circ} \mathrm{C}$ in this solution containing elastase I $(0.28$ $\mathrm{mg} / \mathrm{ml}$ ) for $5 \mathrm{~min}$. Thereafter, preparations were incubated at $37^{\circ} \mathrm{C}$ in a low- $\mathrm{Ca}^{2+}$ $\left(10^{-5} \mathrm{M}\right)$ PSS containing collagenase I $(1 \mathrm{mg} / \mathrm{ml})$, collagenase $\mathrm{XI}(1 \mathrm{mg} / \mathrm{ml})$, papain $(0.15 \mathrm{mg} / \mathrm{ml})$, and dithiothreitol $(1.5 \mathrm{mg} / \mathrm{ml})$ for an additional $5 \mathrm{~min}$. Afterwards, tissues were washed in $\mathrm{Ca}^{2+-}$-free PSS and disaggregated with a 
wide-bore, smooth-tipped pipette. Cells were then placed in a perfusion chamber on the stage of an inverted microscope. After a brief period of time ( $10 \mathrm{~min})$ to allow cells to adhere to the bottom of the chamber, cells were superfused with hypoxic $\mathrm{Ca}^{2+}$-free PSS (in the absence of sodium dithionite) at a rate of $2 \mathrm{ml} / \mathrm{min}$. Hypoxia was induced as described in Determination of reactive oxygen species. Cells were kept in hypoxia for at least $30 \mathrm{~min}$ before initiation of the experiment. Normoxia was established by bubbling the solution with room air. Membrane currents were recorded with the whole cell configuration of the patch-clamp technique, normalized for cell capacitance and expressed in picoamperes per picofarads as previously described $(12,13)$. Currents were recorded under essentially $\mathrm{Ca}^{2+}$-free conditions with an external $\mathrm{Ca}^{2+-}$-free PSS (see above) and a $\mathrm{Ca}^{2+-}$ free pipette (internal) solution containing (in mmol/l) $110 \mathrm{KCl}, 1.2 \mathrm{MgCl}_{2}, 5 \mathrm{Na}_{2}$ ATP, $10 \mathrm{HEPES}, 10 \mathrm{EGTA}, \mathrm{pH}$ adjusted to 7.3 with $\mathrm{KOH}$. EGTA and ATP were included in the pipette solution to minimize the component of ATP-dependent and $\mathrm{Ca}^{2+}$-activated $\mathrm{K}^{+}$currents. Under these conditions, currents were evoked after the application of 200-ms depolarizing pulses from $-60 \mathrm{mV}$ to test potentials from $-60 \mathrm{mV}$ to $+60 \mathrm{mV}$ in $10-\mathrm{mV}$ increments.

\subsubsection{Western blot analysis}

After dissection, DA were immediately frozen in liquid nitrogen and homogenized as previously described (13). The protein content was determined with the Bradford assay (reagents from Bio-Rad). Western blotting was performed with $20 \mu \mathrm{g}$ of protein per lane. SDS-PAGE (7.5\% acrylamide) electrophoresis was performed with the method of Laemmli in a minigel system (Bio-Rad). Samples from mature pDA, immature pDA, and mature aDA were run in parallel. The proteins were transferred to polyvinylidene difluoride (PVDF) membranes overnight at $4^{\circ} \mathrm{C}$ and incubated with rabbit anti-Kv1.5 primary antibody and then with horseradish peroxidase-conjugated anti-rabbit secondary antibody. The bands were visualized by chemiluminescence (ECL, Amersham). Results are expressed relative to a-actin expression in each sample.

\subsubsection{RT-PCR analysis}

Total RNA was isolated and purified from DA homogenates with the RNeasy Fibrous Tissue Mini kit (Qiagen). Total RNA was reverse transcribed into cDNA with the iScript cDNA Synthesis Kit (Bio-Rad) according to manufacturer's instructions. Real-time PCR was performed with a Taqman system (RocheApplied Biosystems, Mannheim, Germany) in the Unidad de Genomica, 
Universidad Complutense de Madrid. Specific primers were designed for chicken superoxide dismutase (SOD)1 (right 5'-GGTCCGGTAAGAGAAATGACAG-3' and left 5'-GACCTCGGCAATGTGACTG-3'), SOD2 (right 5'-ATATGACCCCCACCATTGAA-3' and left 5'-GCTGGCAAAAGGTGATGTTAC-3'), catalase (right 5'-TGGATCCTTCAAATGAGTCTGA-3' and left 5'-GATGCAATGTTGTTTCCATCC-3'), and GAPDH (right 5'-ACCATGTAGTTCAGATCGATGAAG- 3' and left 5'-GTCCTCTCTGGCAAAGTCCA-3').

\subsubsection{Data analysis}

Values are expressed as means \pm SE. Contractions are expressed in terms of active wall tension $(\mathrm{N} / \mathrm{m})$, calculated as the force divided by twice the length of the segment. Relaxations are expressed as a percentage of decrease of the initial tone previously induced by $\mathrm{O}_{2}, t$-butyl-hydroperoxide, or $\mathrm{KCl}$. Sensitivity [expressed as apparent affinity $\left(\mathrm{pD}_{2}\right)=-\log \mathrm{EC}_{50}$ ] to agonists was determined for each artery by fitting individual concentration-response data to a nonlinear sigmoidal regression curve (GraphPad Prism version 2.01; GraphPad Software, San Diego, CA). Differences between mean values were assessed by one-way ANOVA followed by Bonferroni post hoc $t$-test. Nonpaired $t$-tests were used if only two groups were compared. Differences were considered significant at $\mathrm{P}<$ 0.05 .
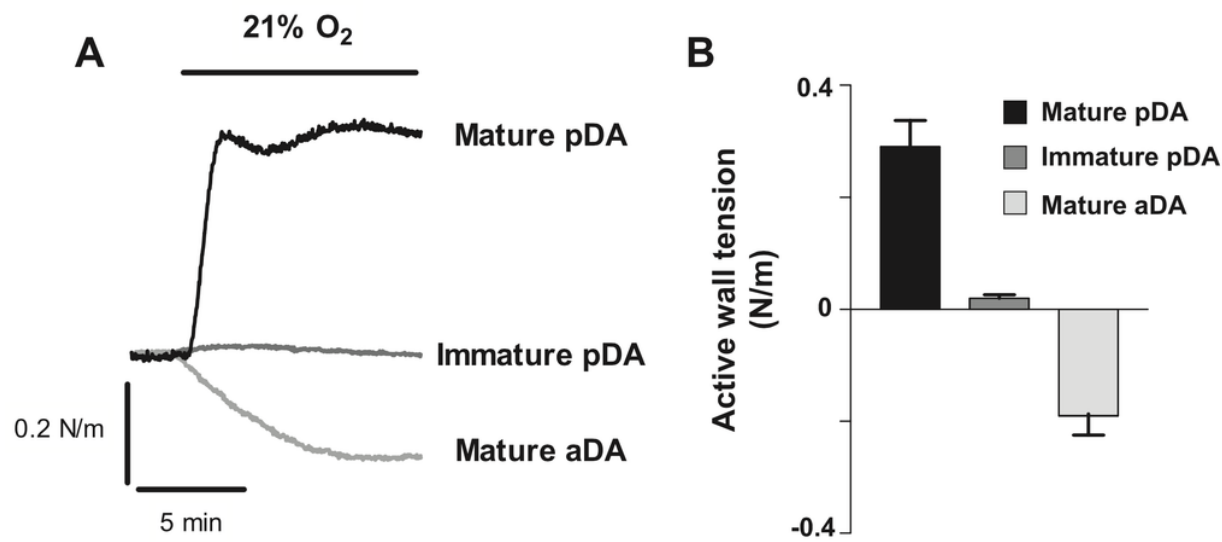

Fig. 2.1. Heterogeneity of oxygen-induced response in ductus arteriosus (DA) preparations. Representative traces $(A)$ and mean data $(B)$ showing the responses induced bij $21 \% \mathrm{O}_{2}$ in mature pulmonary DA (pDA), immature pDA, and mature aortic DA (aoDA). Results are means $\pm S ; n=6-9$. 

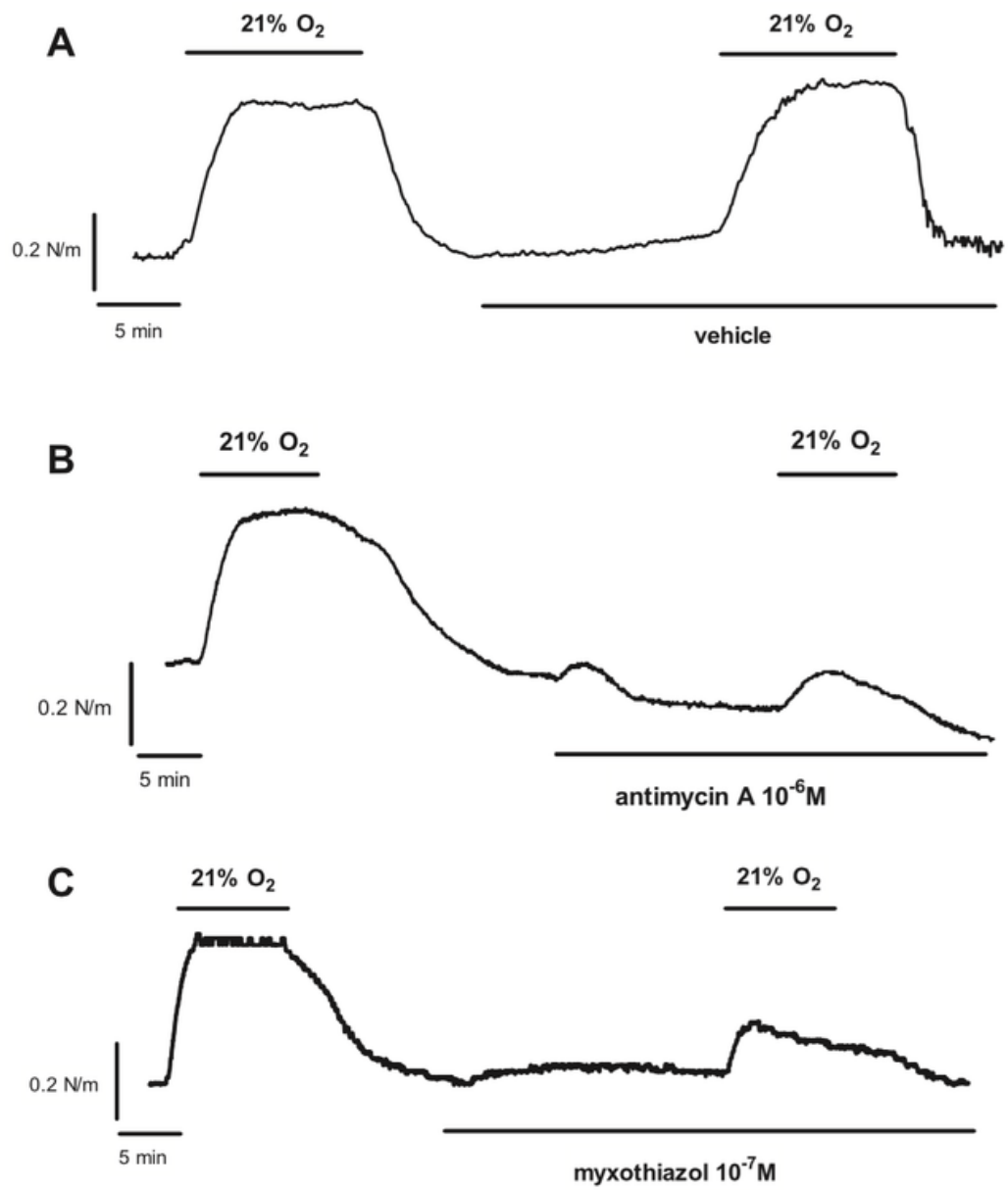

D

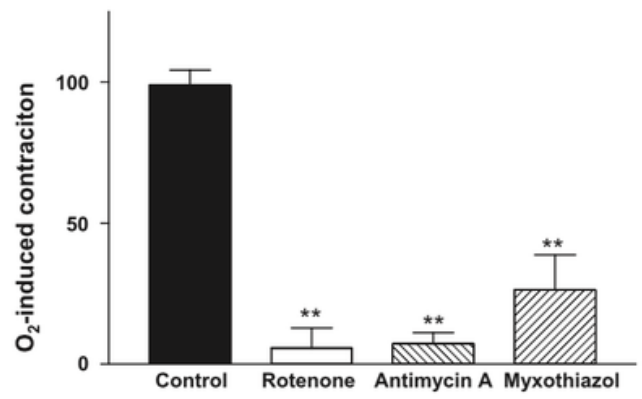

Fig. 2.2. Inhibition of mitochondrial electron transport chain (ETC) blunts $\mathrm{O}_{2}$-induced constriction in mature pDA. Representative traces $(A-C)$ and mean data $(D)$ show the responses induced bij $21 \%$ $\mathrm{O} 2$ in the absence (control) or in the presence of complex I (rotenone) or complex III (myxothiazol and antimycin A) inhibitors. Results are means \pm SE; $n=5-9$. ${ }^{*} P<0.01$ vs. control (ANOVA followed by Bonferroni's test). 


\subsection{Results}

\subsection{1 $\mathrm{O}_{2}$-induced contractions and effects of ETC inhibitors}

After an equilibration period of $45 \mathrm{~min}$ in hypoxia $\left(0 \% \mathrm{O}_{2}\right)$, vessels were exposed to normoxia $\left(21 \% \mathrm{O}_{2}\right)$ for $10 \mathrm{~min}$. $\mathrm{O}_{2}$ induced a contractile response in mature pDA but not in immature pDA as we had previously reported (1), whereas in mature aDA it even caused a relaxant response (Fig. 2.1). $\mathrm{O}_{2-}$ induced contraction in mature pDA was easily reversible when returning to hypoxia and highly reproducible in two consecutive challenges (Fig. 2.2A). Thus each vessel was exposed twice to normoxia. To test the role of the mitochondrial ETC, the second challenge to normoxia was elicited in the absence or presence of inhibitors. The contraction induced by $\mathrm{O}_{2}$ was abolished by rotenone $\left(10^{-6} \mathrm{M}\right)$, an inhibitor of complex I of the mitochondrial ETC. However, at this concentration rotenone had no effect on the contraction induced by the thromboxane $A_{2}$ mimetic U-46619 (102 $\pm 2 \%$ and $96 \pm 3 \%$ of a previous stimulation with $\mathrm{U}-46619$, in the absence and the presence of rotenone, respectively; $P>0.05, n=4-5)$. Inhibition of the ETC complex III with antimycin $A\left(10^{-6} \mathrm{M}\right)$ or myxothiazol $\left(10^{-7} \mathrm{M}\right)$ also blunted $\mathrm{O}_{2}$-induced constriction (Fig. 2). At the concentrations used, antimycin A induced a slight decrease (Fig. 2.2B) and myxothiazol a slight increase (Fig. 2.2C) of the basal tone.

\subsubsection{Role of ROS as mediators}

Mature pDA exhibited an increase in DHE fluorescence following 5-min exposure to $21 \% \mathrm{O}_{2}$, and this effect was prevented by the SOD mimetic tiron $\left(10^{-5} \mathrm{M}\right)$, indicating increased superoxide generation (Fig. 2.3A). Thereafter, we tested the effects of normoxia in DA incubated with DCF, a dye widely used for detecting $\mathrm{H}_{2} \mathrm{O}_{2}$. A marked elevation in DCF fluorescence was found in mature pDA after normoxic challenge (Fig. 2.3, $B$ and $C$ ). This effect was evident within the first minute of normoxic challenge. In contrast, normoxia did not increase DCF fluorescence in either immature pDA or mature aDA.

We also examined the mRNA expression of SOD1, SOD2, and catalase (Fig. 2.4). Compared with immature pDA, mature pDA had lower expression of SOD1 but similar expression of SOD2 and catalase. SOD1 and SOD2 were similarly expressed in mature $\mathrm{pDA}$ and $\mathrm{aDA}$, whereas catalase was twofold higher in mature aDA than in pDA. In addition, the contraction of mature pDA evoked by normoxia in the presence of the membrane-permeant analog of the $\mathrm{H}_{2} \mathrm{O}_{2}$ scavenger catalase [polyethylene glycol (PEG)-catalase $100 \mathrm{U} / \mathrm{ml}$ ] only reached $51 \pm 12 \%$ of that obtained under control conditions. 
A

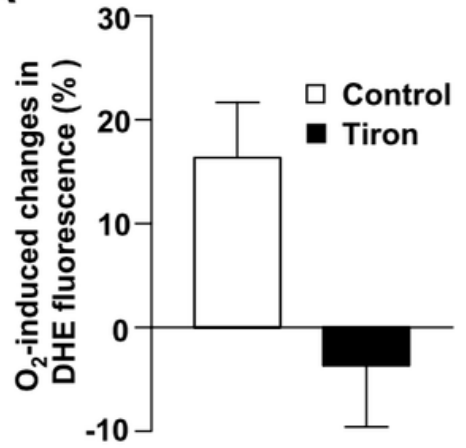

C
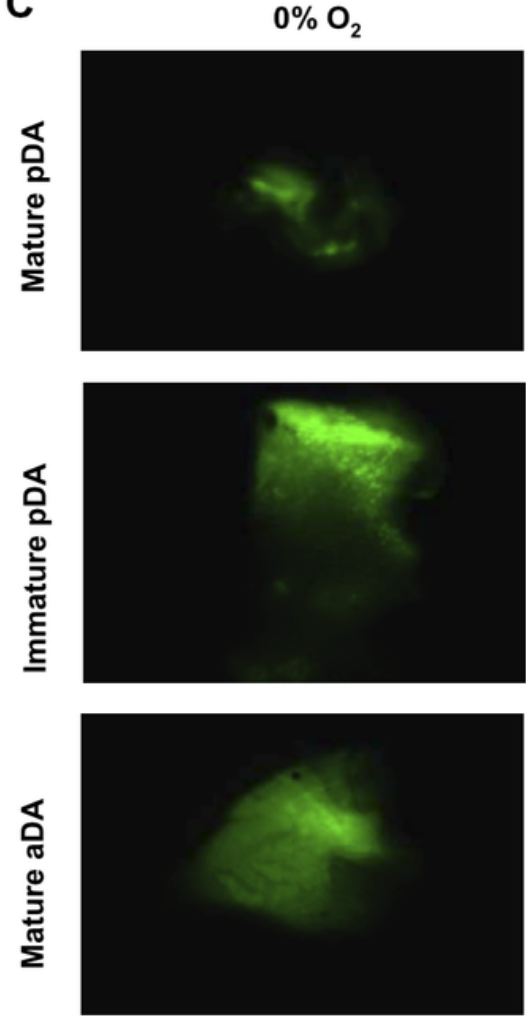

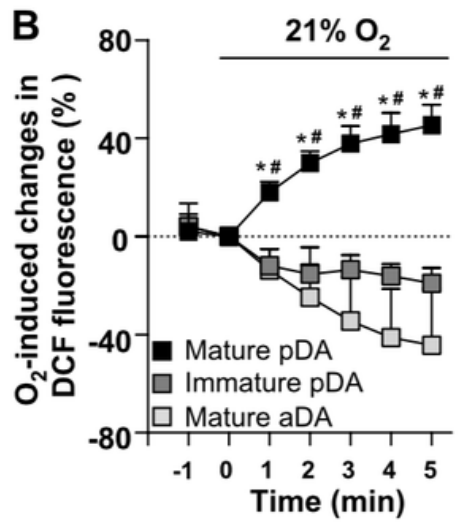

$21 \% \mathrm{O}_{2}$
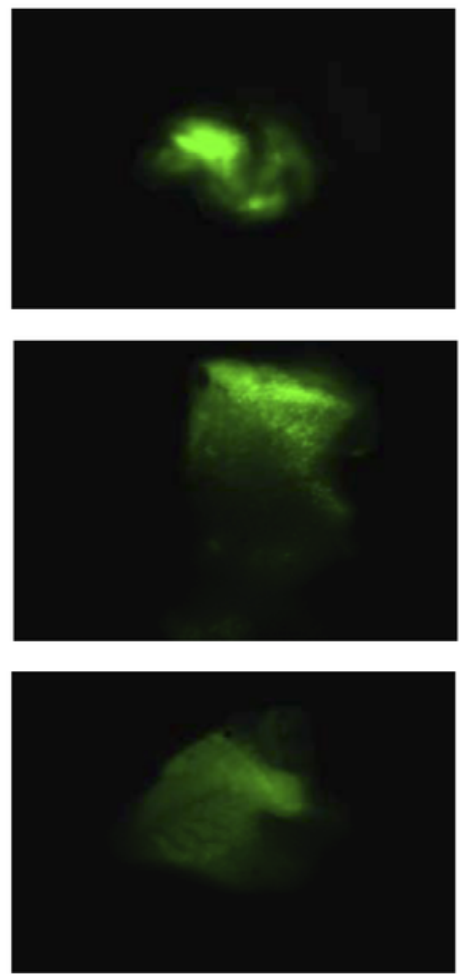

Fig. 2.3. Normoxia selectively increases reactive oxygen species (ROS) production in mature pDA. $A$ : changes in dihydroethidium (DHE) fluorescence induced by normoxie in mature pDA incubated with vehicle (control) or the superoxide dismutase mimetic tiron $\left(10^{-5} \mathrm{M}\right)$. $B$ and $C$ : time course $(B)$ and representative pictures $(C)$ of the changes in 2',7'-dichlorofluorescein (DCF) fluorescence following exposure to $21 \% \mathrm{O}_{2}$ in mature $\mathrm{pDA}$, immature $\mathrm{pDA}$, and mature aDA. Pictures were taken from the same vessel before and after 5-min exposure to normoxia. Results are means $\pm \mathrm{SE} ; n=$ 3-6. $P<0.05$ vs. *immature pDA and \#mature aDA (ANOVA followed by Bonferroni's test). 
In the next set of experiments, the effect of the membranepermeant $\mathrm{H}_{2} \mathrm{O}_{2}$ analog $t$-butyl-hydroperoxide $\left(10^{-5} \mathrm{M}-10^{-4} \mathrm{M}\right)$ was tested. The $\mathrm{H}_{2} \mathrm{O}_{2}$ analog had no contractile effect on immature pDA but caused a concentration-dependent contraction of mature pDA (Fig. 2.5, $A$ and $C$ ). In contrast, $t$-butylhydroperoxide relaxed mature aDA (Fig. 2.5, $B$ and $C$ ) in a concentration-dependent manner. Therefore, application of the $\mathrm{H}_{2} \mathrm{O}_{2}$ analog mimicked the effects of normoxia on the three vessels.

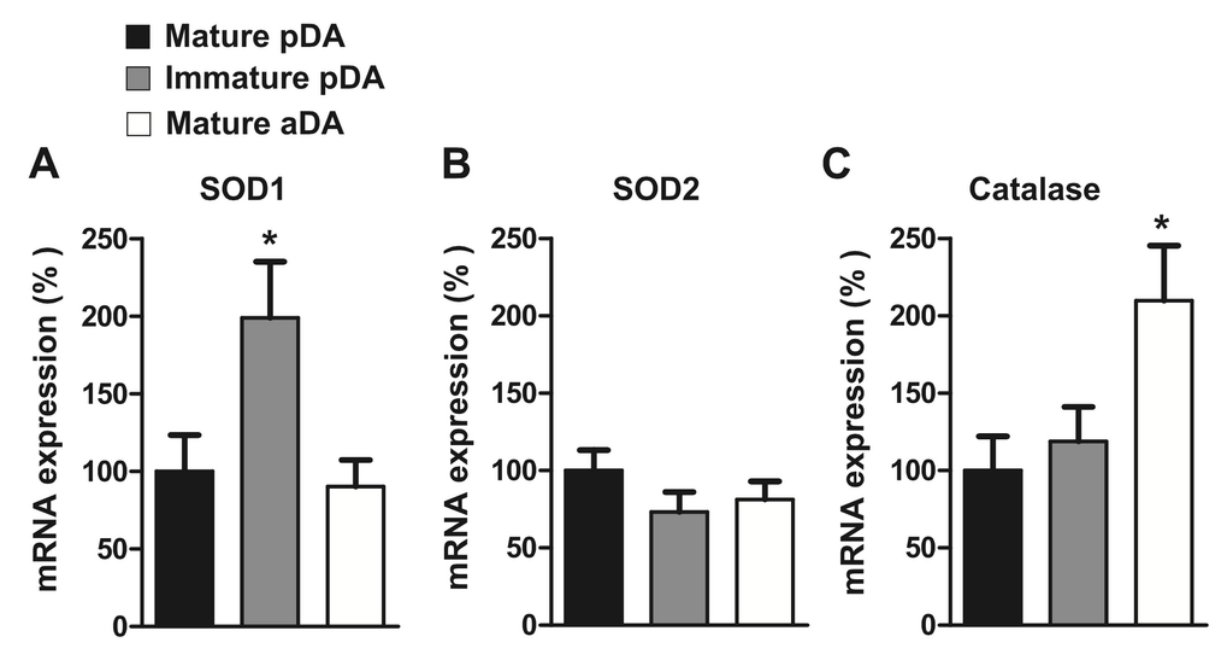

Fig. 2.4. Expression of superoxide dismutase (SOD) $1(A)$, SOD2 $(B)$, and catalase $(C)$ analyzed by RT-PCR. Results are normalized to GAPDH and represent means $\pm \mathrm{SE} ; n=6$. ${ }^{*} P<0.05$ vs. mature pDA (ANOVA followed by Bonferroni's test).

\subsubsection{Role of voltage-gated potassium channels as effectors}

Addition of the $\mathrm{K}_{v}$ channel blocker 4-aminopyridine (4-AP, 10-2 M) contracted immature $\mathrm{pDA}$ and mature $\mathrm{pDA}$ and $\mathrm{aDA}$. The contractile responses induced by the $\mathrm{K}_{v}$ channel blocker were significantly smaller in immature pDA $(0.14 \pm 0.01$ $\mathrm{N} / \mathrm{m}, n=5)$ compared with mature pDA $(0.43 \pm 0.04 \mathrm{~N} / \mathrm{m}, n=12)$ and $\mathrm{aDA}(0.48$ $\pm 0.04 \mathrm{~N} / \mathrm{m}, n=8$ ). However, no significant differences were found between vessels when values were expressed as a percentage of a previous response to $\mathrm{KCl}(79 \pm 11 \%, 67 \pm 6 \%, 82 \pm 10 \%$ for immature $\mathrm{pDA}$, mature pDA, and mature aDA, respectively).

The possible modulation of $\mathrm{Kv}$ channels by normoxia was tested in isolated DASMC with the patch-clamp technique. Potassium current amplitudes were about threefold higher in cells isolated from mature pDA than in those from 
immature pDA (Fig. 2.6A). Currents present in cells from mature pDA were markedly inhibited by normoxia, whereas no effects were observed in DASMC isolated from immature pDA (Fig. 2.6, $B$ and $C$ ). Addition of the $\mathrm{K}_{v}$ channel blocker 4-AP $\left(10^{-3} \mathrm{M}\right)$ inhibited the current to a similar extent in mature and immature DASMC (Fig. 2.6, $D$ and $E$ ), whereas at $10^{-2} \mathrm{M}$ the potassium current in mature pDA cells was nearly abolished (not shown). Moreover, in the presence of 4-AP $\left(10^{-3} \mathrm{M}\right)$, the effects of normoxia in mature pDA cells were prevented. To test whether the difference in the effect of normoxia on potassium currents was caused by different Kv1.5 protein content, we analyzed its expression in immature pDA, mature pDA, and mature aDA. Surprisingly, the expression of $\mathrm{K}_{\mathrm{v}} 1.5$ channels was lower in mature pDA than in immature pDA or mature aDA (Fig. 2.6, $F$ and $G$ ).

\subsubsection{Role of $\mathrm{Ca}^{2}$ channels as effectors}

The inhibitory effect of normoxia on $\mathrm{K}_{\mathrm{V}}$ currents is expected to activate L-type $\mathrm{Ca}^{2}\left(\mathrm{Ca}_{\mathrm{L}}\right)$ channels, contributing to $\mathrm{O}_{2}$-induced constriction. Consistent with this, incubation with the CaL channel blocker nifedipine inhibited the contractile response to $\mathrm{O}_{2}$ in mature pDA (Fig. 2.7). Additionally, $\mathrm{O}_{2}$-induced constriction was nearly blunted in the absence of extracellular calcium. Nifedipine also relaxed pDA previously contracted with $\mathrm{O}_{2}$. Interestingly, nifedipine caused a similar relaxant response in mature pDA contracted with $21 \% \mathrm{O}_{2}$ or with $t$-butylhydroperoxide (10-4 M) (Fig. 2.7D).

In another set of experiments, DA were initially contracted with $\mathrm{KCl}\left(62.5 \times 10^{-3}\right.$ $M)$, and thereafter nifedipine was added in a cumulative fashion. A concentration of $10^{-5} \mathrm{M}$ nifedipine was needed to achieve a near-full relaxant effect in $\mathrm{KCl}$ contracted mature pDA (Fig. 2.8A). Moreover, immature pDA were less sensitive to nifedipine than mature $\mathrm{pDA}$. To test whether this difference was due to a maturation of CaL channels we tested the effects of the CaL opener BAY K8644. This drug had no effects in immature pDA but contracted mature pDA in a concentration-dependent manner (Fig. 2.8B). This contractile response was suppressed in the presence of nifedipine (not shown). Finally, pretreatment with BAY K8644 did not modify the contractile response to $\mathrm{O}_{2}$ in mature $(98 \pm 11 \%$ of the response to oxygen in the absence of the drug, $\mathrm{P}<0.05$; Fig. $2.8 \mathrm{C}$ ) or immature (Fig. 2.8D) pDA. 
A

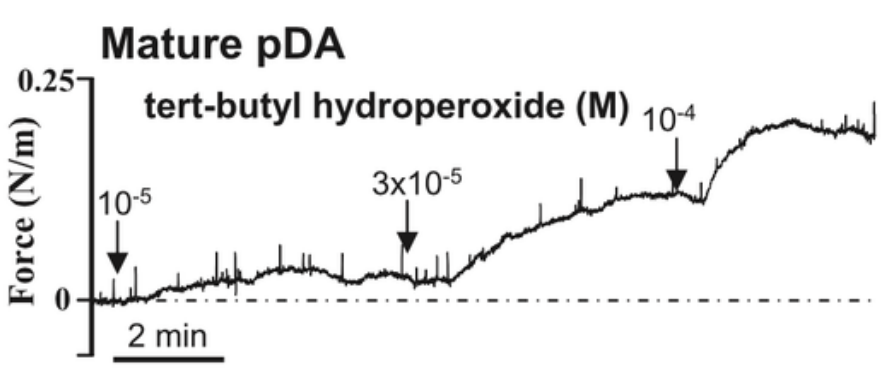

B

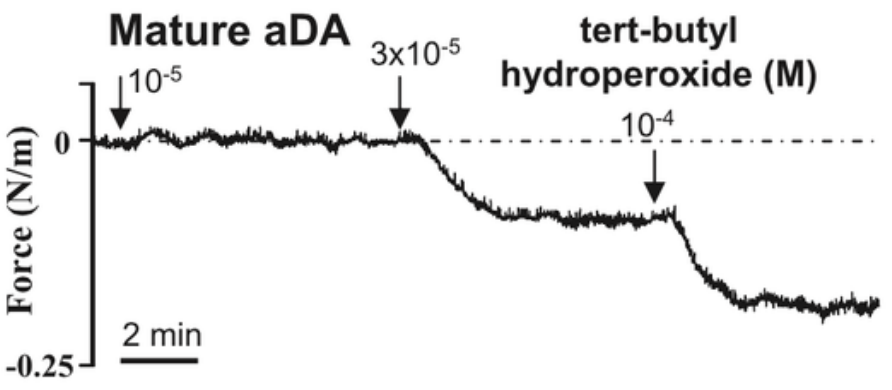

C

tert-butyl hydroperoxide (M)

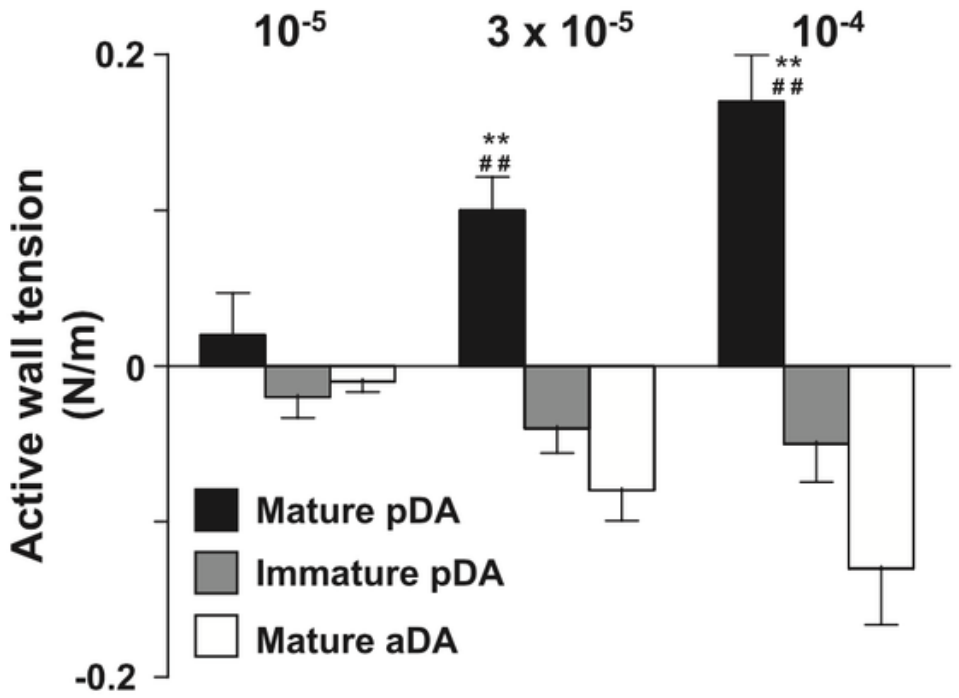

Fig. 2.5. $\mathrm{H}_{2} \mathrm{O}_{2}$ analog $t$-butyl-hydroperoxide mimics the effects of normoxia in chicken DA. Representative pictures $(A$ and $B$ ) and mean data $(C)$ show responses induced by $t$-butylhydroperoxide $\left(10^{-5}-10^{-4} \mathrm{M}\right)$ in mature pDA, immature pDA, and mature aDA. Results are means \pm $\mathrm{SE} ; n=4-6 . P<0.01$ vs. ${ }^{*}$ immature pDA and ${ }^{\#}$ mature aDA (ANOVA followed by Bonferroni's test). 
A

\section{Immature pDA}

Hypoxia

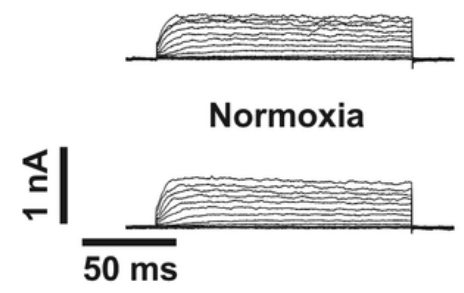

B
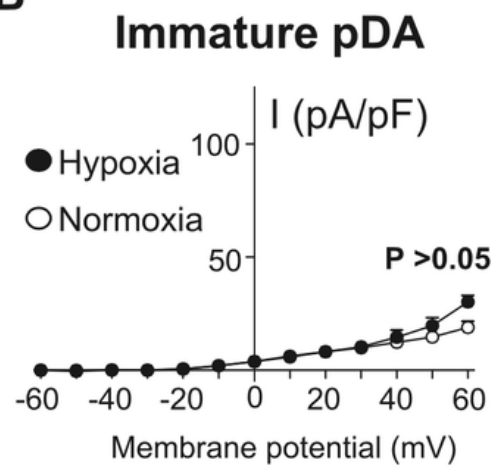

D

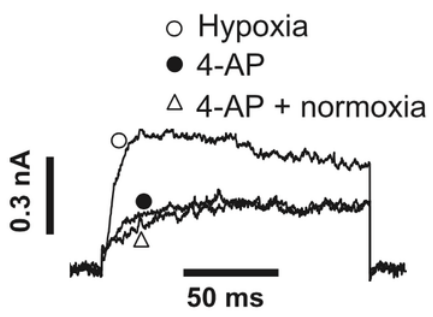

F

Immat. Mat. Mat. pDA pDA aDA

Kv1.5

a-actin

\section{Mature pDA}

Hypoxia

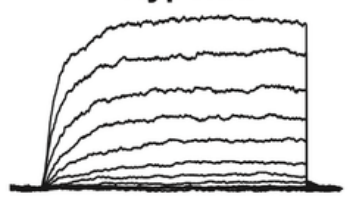

Normoxia

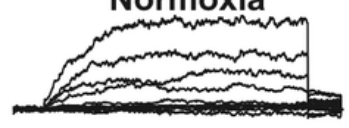

C

Mature pDA
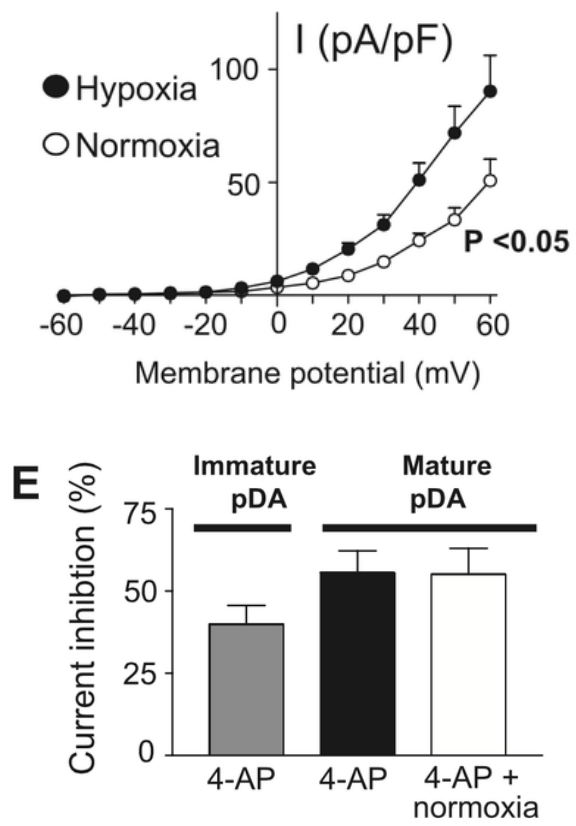

G

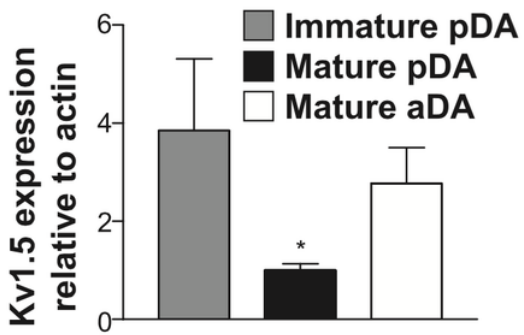


Fig. 2.6. Normoxia inhibits voltage-gated potassium $\left(K_{v}\right)$ currents in DA smooth muscle cells (DASMC) isolated from mature but not from immature pDA. A: potassium current traces are shown for depolarization pulses from $-60 \mathrm{mV}$ to $+60 \mathrm{mV}$ from a holding potential of $-60 \mathrm{mV}$ in mature and immature pDASMC before (hypoxia) and after 5 min under normoxic conditions. $B$ and $C$ : current (I)-voltage relationships of potassium currents recorded in immature $(n=4)$ or mature $(n=6)$ pDASMC) under hypoxic or normoxic conditions. $D$ : potassium current trace from a mature DASMC elicited when stepping to $+30 \mathrm{mV}$ under hypoxic conditions in the absence or the presence of 4aminipyridine (4-AP, 10-3 M). Subsequent switch to normoxic solution in the continuous presence of 4-AP was without effect. E: mean data of the inhibitory effects of 4-AP or 4-AP + normoxia on the current elicited at $+30 \mathrm{mV}$ in mature or immature pDASMC. $F$ and $G$ : protein expression go $\mathrm{K}_{\mathrm{v}} 1.5$ channels analyzed by Western blot in immature pDA, mature pDA, and mature aDA. Blots were reprobed with smooth muscle a-actin as a loading control. Results are normalized to a-actin. ${ }^{*} P<$ 0.05 vs. mature aDA and immature pDA.

\subsubsection{Role of Rho kinase as effector}

In mature pDA stimulated with $21 \% \mathrm{O}_{2}$, the addition of Rho kinase inhibitors hydroxyfasudil or Y-27632 induced a concentration-dependent relaxation (Fig. 2.9). Interestingly, contractions induced by $\mathrm{O}_{2}$ and $t$-butyl-hydroperoxide showed a similar sensitivity to hydroxyfasudil $\left(\mathrm{pD}_{2}=5.82 \pm 0.16\right.$ and $5.68 \pm 0.176$, respectively, $\mathrm{P}>0.05$; Fig. $2.9 \mathrm{C})$ and to $\mathrm{Y}-27632\left(\mathrm{pD}_{2}=5.92 \pm 0.16\right.$ and $5.79 \pm$ 0.2 , respectively, $\mathrm{P}>0.05$; Fig. $2.9 \mathrm{D}$ ). In the last series of experiments, we examined the relaxation induced by the two Rho kinase inhibitors in mature $\mathrm{pDA}$, mature aDA, and immature pDA stimulated with $\mathrm{KCl}$. The potency of hydroxyfasudil (Fig. 2.9E) and Y-27632 (Fig. 2.9F) was higher in mature pDA $\left(\mathrm{pD}_{2}=5.75 \pm 0.13\right.$ and $6.37 \pm 0.1$, respectively) than in immature $\mathrm{pDA}\left(\mathrm{pD}_{2}=\right.$ $5.22 \pm 0.11$ and $5.51 \pm 0.13$, respectively; $P<0.05$ vs. mature pDA for both drugs). Moreover, both drugs showed a similar potency to relax mature aDA $\left(\mathrm{pD}_{2}=5.71 \pm 0.14\right.$ and $6.11 \pm 0.13$ for hydroxyfasudil and $\mathrm{Y}-27632$, respectively) compared with pDA (data above). 

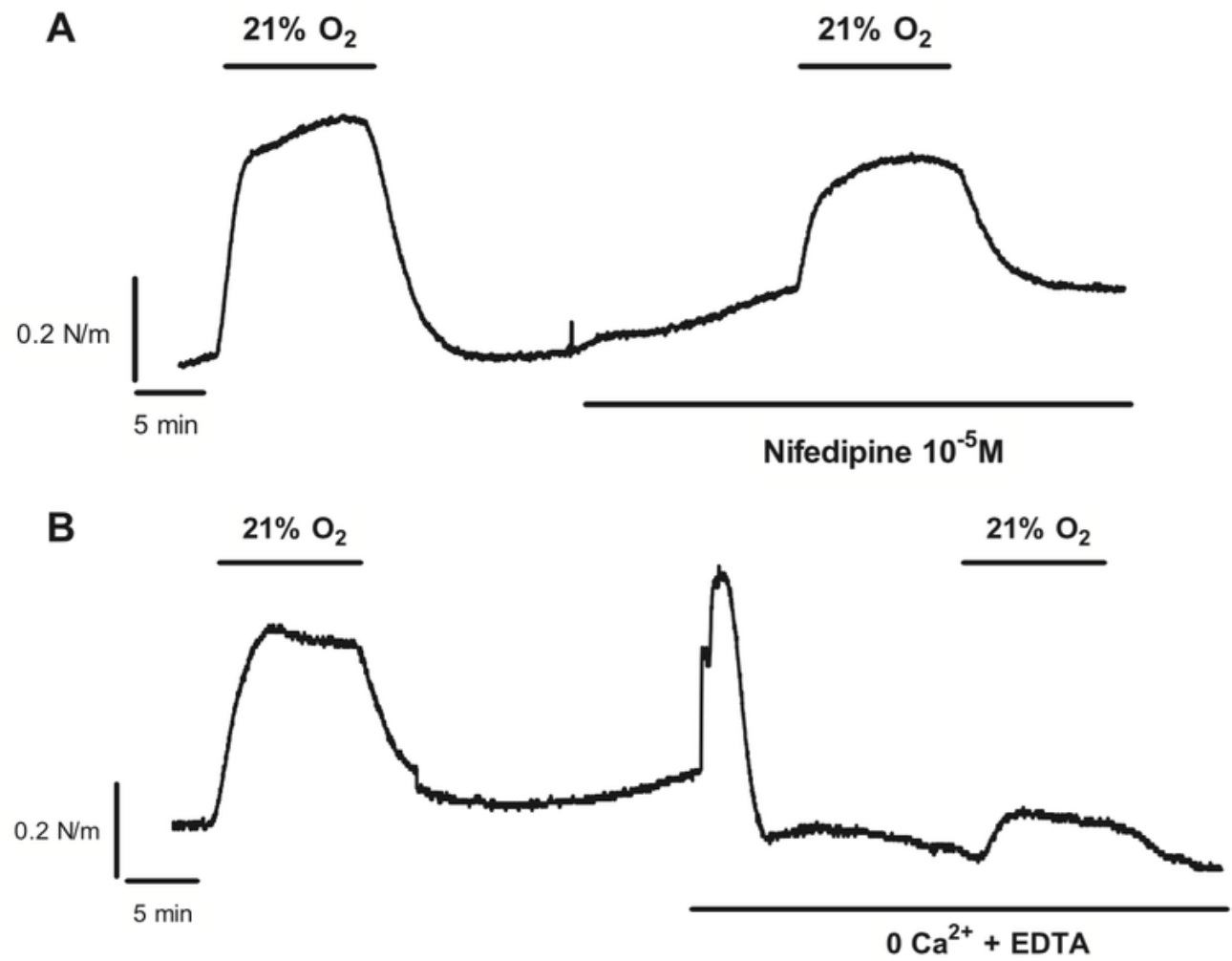

C

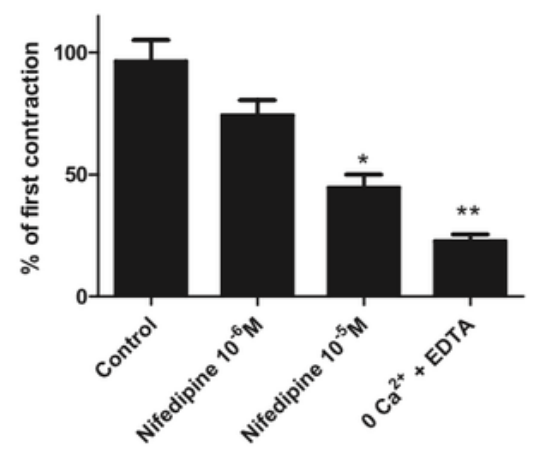

D

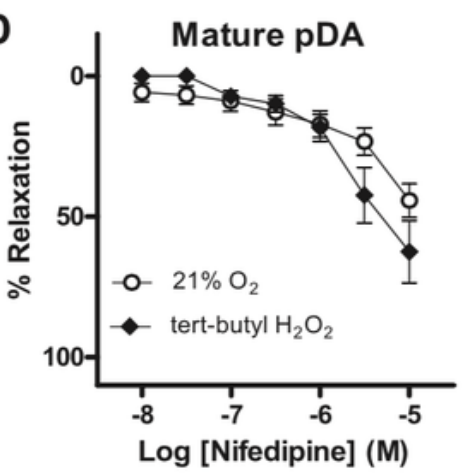

Fig. 2.7. Inhibition of L-type $\mathrm{Ca}^{2+}(\mathrm{Ca} L)$ channels attenuates $\mathrm{O}_{2}$-induced constriction. $A$ and $B$ : representative tracings showing 2 consecutive responses to $21 \% \mathrm{O}_{2}$ in mature pDA. The second exposure to $\mathrm{O}_{2}$ was elicited after incubation with nifedipine $(A)$ or $0 \mathrm{Ca}^{2+}+$ EDTA $(B)$. C: mean data of the second contraction induced by $21 \% \mathrm{O}_{2}$ (expressed as $\%$ of $1^{\text {st }}$ response) after incubation with vehicle (control), nifedipine, or $0 \mathrm{Ca}^{2+}+$ EDTA. $D$ : concentration-dependent relaxant responses of nifedipine in mature pDA stimulated with $21 \% \mathrm{O}_{2}$ or $t$-butyl-hydroperoxide $\left(10^{-4} \mathrm{M}\right)$. Responses are means \pm SE; $n=5-6$. ${ }^{*} P<0.05,{ }^{*} P<0.01$ vs. control (ANOVA followed by Bonferroni's test). 

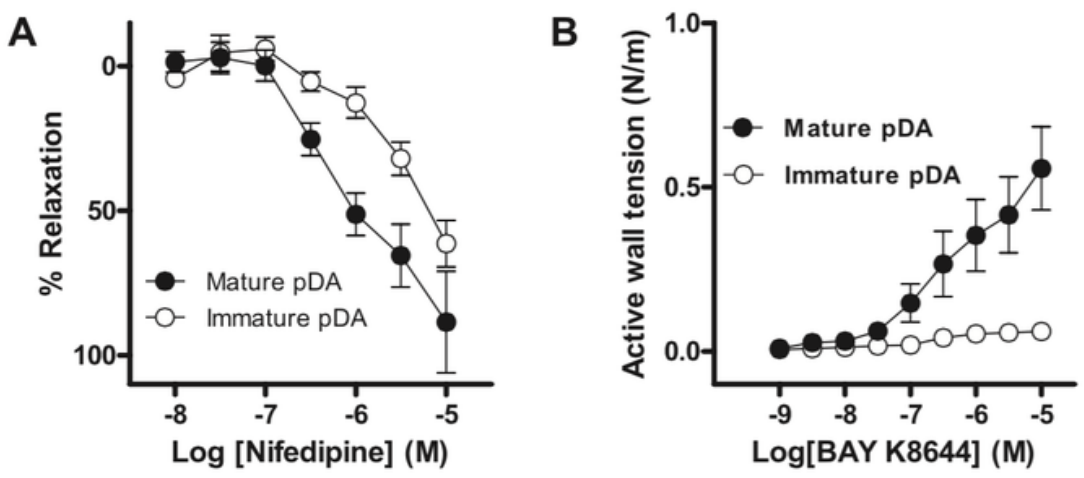

C

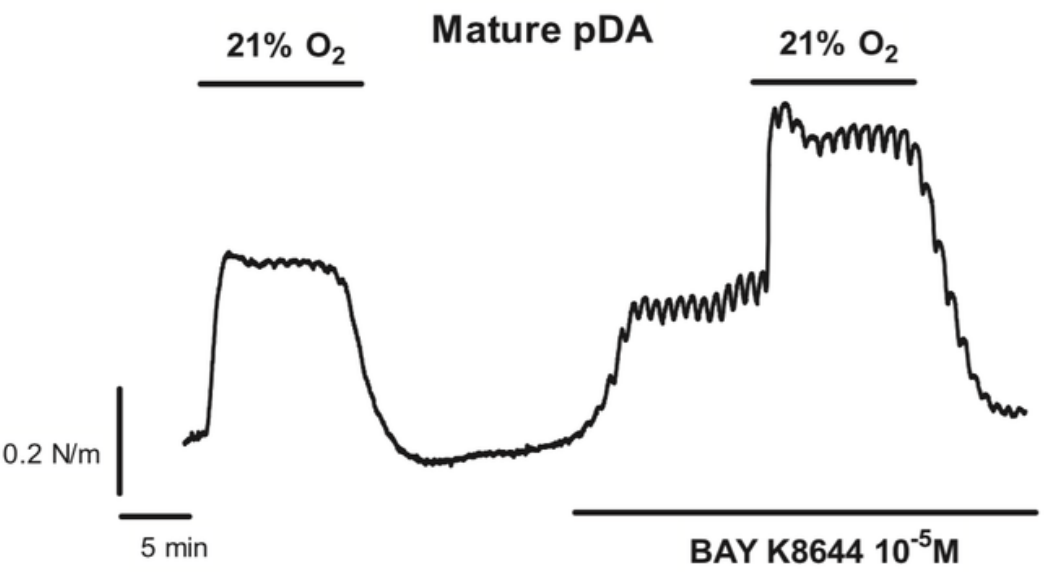

D Immature pDA

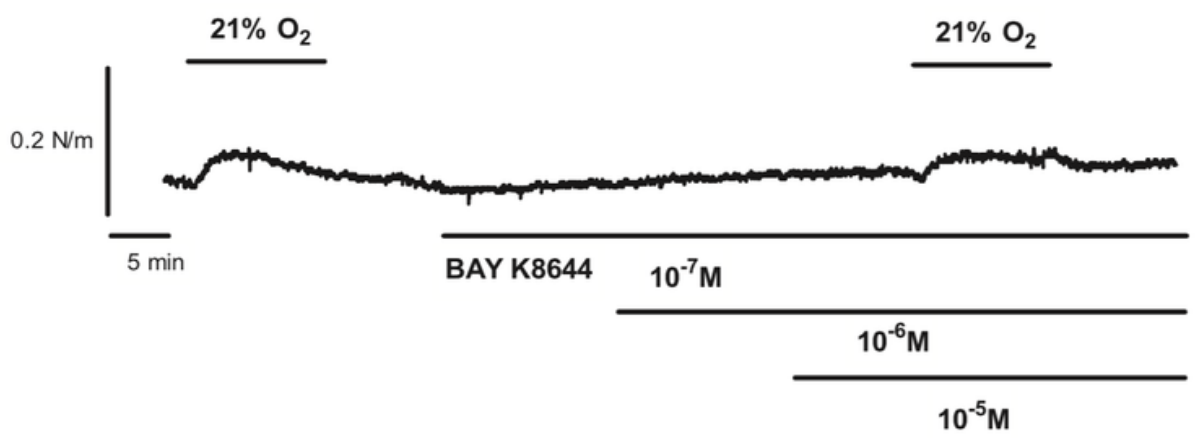

Fig. 2.8. Maturational changes in the response to dihydropyridines. $A$ and $B$ : concentrationdependent responses to nifedipine $(A)$ and BAY $\mathrm{K} 8644(B)$ in immature and mature pDA. Experiments were performed under hypoxic conditions, and the relaxant responses to nifedipine were tested in DA stimulated with $\mathrm{KCl}\left(62.5 \times 10^{-3} \mathrm{M}\right) . C$ and $D$ : representative traces showing the inability of BAY K8644 to affect responses to $\mathrm{O}_{2}$ in mature $(C)$ and immature $(D)$ pDA. Results are means \pm SE; $n=5-9$. 

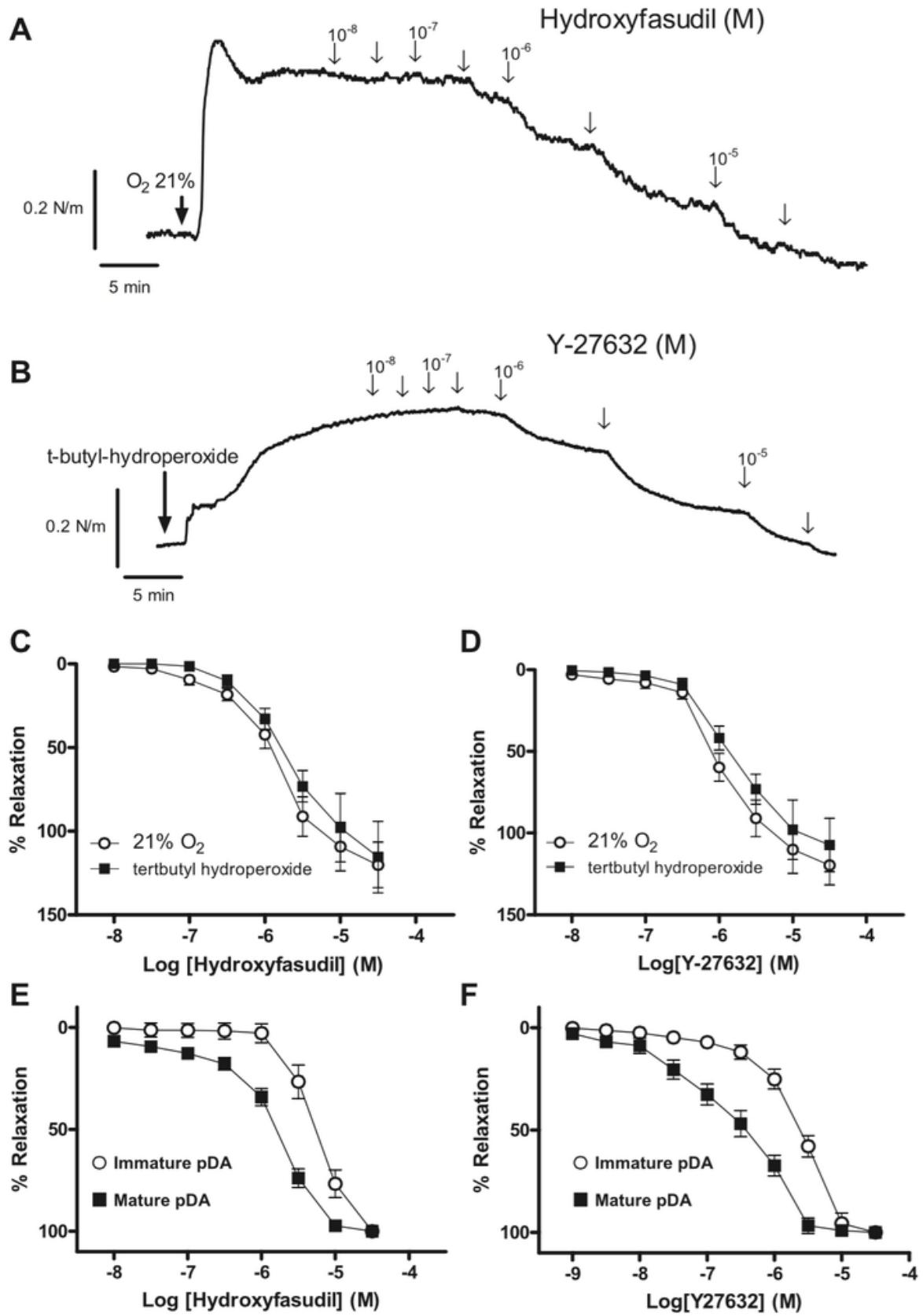

Fig. 2.9. Rho kinase inhibition reverses $\mathrm{O}_{2}$ and $t$-butyl-hydroperoxide constriction in mature pDA. $A$ $D$ : representative traces and mean data showing that hydroxyfasudil $(A$ and $C)$ and $Y-27632$ ( $B$ and $D$ ) relaxed the contraction induced by $21 \% \mathrm{O}_{2}$ and $t$-butyl-hydroperoxide $\left(10^{-4} \mathrm{M}\right)$ in a concentrationdependent manner. $E$ and $F$ : relaxant responses induced by hydroxyfasudil $(E)$ and $\mathrm{Y}-27632(F)$ in immature pDA and mature pDA stimulated with $\mathrm{KCl}\left(62.5 \times 10^{-3} \mathrm{M}\right)$. Results are means $\pm \mathrm{SE} ; n=$ 6-8. 


\subsection{Discussion}

The preparation of the DA for its specific task of postnatal closure is reflected in critical maturational changes in reactivity $(1-3,9,29)$. The chicken lacks the limitations of most available models and has arisen as an excellent model for studying the developmental biology of the DA (31). In the present study, we have examined the developmental changes in the cellular mechanisms (sensor, mediator, and effectors) involved in the chicken DA response to $\mathrm{O}_{2}$. Our results can be summarized as follows:

1) Contraction induced by $\mathrm{O}_{2}$ is restricted to the mature pulmonary side of the DA.

2) This response is nearly abolished by mitochondrial ETC inhibitors.

3) Normoxia increases ROS production in the pulmonary side of mature DA but not in the immature DA or in the aortic side.

4) Exogenous addition of the $\mathrm{H}_{2} \mathrm{O}_{2}$ analog $t$-butyl-hydroperoxide mimics the effects of normoxia in all vessels tested.

5) $\mathrm{Kv}$ currents change during development from low-amplitude $\mathrm{O}_{2}$ insensitive currents in immature $\mathrm{pDA}$ to high-amplitude $\mathrm{O}_{2}$-sensitive currents in mature pDA.

6) The inhibition of $\mathrm{Ca}_{\mathrm{L}}$ channels and Rho kinase attenuates the normoxic constriction.

\subsubsection{Mitochondria as sensor}

Mitochondria have been proposed as $\mathrm{O}_{2}$ sensors in several specialized $\mathrm{O}_{2}$ sensing tissues such as the resistance pulmonary arteries, glomus cells of the carotid body, fetal adrenomedullary chromaffin cells, and the DA $(4,14,23,37$, 38, 40). Michelakis et al. (23) reported that complex I and complex III ETC inhibitors fully relaxed $\mathrm{O}_{2}$ constriction in human DA. In the chicken DA, the inhibition of complex I or III also abolishes the contractile response to $\mathrm{O}_{2}$ (Ref. 16 and present study). The effect of the complex I inhibitor rotenone was selective since the contraction induced by $\mathrm{U}-46619$ was not affected.

$\mathrm{H}_{2} \mathrm{O}_{2}$ as mediator. An increased production of ROS (specifically $\mathrm{H}_{2} \mathrm{O}_{2}$ ) has been proposed to underlie the $\mathrm{O}_{2}$ response in mammalian DA $(18,23,27)$. Here, we found that normoxia augmented fluorescence to DHE, which mainly detects changes in cytosolic concentrations of superoxide. The increase in DHE fluorescence was prevented by the superoxide mimetic tiron, confirming that it was due to the elevation in superoxide levels. Superoxide is rapidly dismuted by SOD to $\mathrm{H}_{2} \mathrm{O}_{2}$, a stable and mobile molecule that is more likely to act as the signaling mediator. Thus we measured the changes in $\mathrm{H}_{2} \mathrm{O}_{2}$ levels by using the 
fluorescent dye DCF. $\mathrm{O}_{2}$ increased the fluorescence to DCF in mature pDA, but not in either mature aDA or immature pDA. Therefore, the three preparations exhibit a profound parallelism in terms of tension generation and $\mathrm{H}_{2} \mathrm{O}_{2}$ production after normoxia. Moreover, $\mathrm{O}_{2}$ induces contraction and increases $\mathrm{H}_{2} \mathrm{O}_{2}$ production in the DA with a similar time course. Another argument in favor of $\mathrm{H}_{2} \mathrm{O}_{2}$ as the $\mathrm{O}_{2}$ mediator in the chicken DA was the marked attenuation of the $\mathrm{O}_{2}$ constriction following the application of the $\mathrm{H}_{2} \mathrm{O}_{2}$ scavenger PEG-catalase. In accordance with this, Greyner and Dzialowski (16) observed that the $\mathrm{H}_{2} \mathrm{O}_{2}$ scavenger $\mathrm{N}$-mercaptopropionylglycine relaxed $\mathrm{O}_{2}$-induced contraction in the mature pDA. Furthermore, in striking similarity with $\mathrm{O}_{2}$, the $\mathrm{H}_{2} \mathrm{O}_{2}$ analog t-butylhydroperoxide contracted mature pDA, had no effect on immature pDA, and relaxed mature aDA. In mammalian $\mathrm{DA}, \mathrm{H}_{2} \mathrm{O}_{2}$ has been shown to induce constriction because of its ability to inhibit $\mathrm{K}_{v}$ channels $(23,27)$ and to activate Rho kinase (18), which are considered effectors of the $\mathrm{O}_{2}$ response (see below). In the chicken DA, nifedipine (which reverses depolarization-induced contraction) and Rho kinase inhibitors relaxed $\mathrm{O}_{2-}$ and $\mathrm{H}_{2} \mathrm{O}_{2}$-induced contraction with a similar potency and efficacy, which is also consistent with the involvement of $\mathrm{H}_{2} \mathrm{O}_{2}$ as the mediator molecule of the $\mathrm{O}_{2}$ response.

\subsubsection{Kv channels as effectors}

In rabbit $(27,39)$ and human $(23) \mathrm{DA}$ it has been reported that $\mathrm{O}_{2}$ inhibits $\mathrm{K}_{\mathrm{v}}$ channels, depolarizes membrane potential, and causes vasoconstriction. In our previous study (1), we found that the chicken DA constricts in response to the $\mathrm{K}_{\mathrm{V}}$ channel inhibitor 4-AP, whereas the inhibition of ATP-dependent and largeconductance $\mathrm{Ca}^{2}$-activated $\mathrm{K}$ channels caused no effect, suggesting that $\mathrm{Kv}$ channels are the predominant $\mathrm{K}$ channels controlling chicken DA tone. Those experiments were performed in the central portion of the DA, which contains a similar proportion of tissue from pulmonary and aortic sides. Here we observed that the inhibition of $\mathrm{K}_{v}$ channels causes a comparable vasoconstriction in the pulmonary and aortic sides of mature DA. Similar results have been recently reported (16). In addition, no differences were found in the response to 4-AP between mature and immature pDA when values were normalized to a previous response to $\mathrm{KCl}$. Altogether these data indicate that under basal conditions $\mathrm{K}_{\mathrm{V}}$ channels make a similar contribution in controlling tone in the chicken DA regardless of gestational age or the portion of the vessel studied.

This is the first study examining potassium currents in the chicken DA. Under our experimental conditions, $\mathrm{K}_{\mathrm{v}}$ channels were the main contributors to the total outward current in both mature and immature pDASMC as evidenced by the sensitivity to 4-AP. Perfusion with normoxic solution dramatically decreased $\mathrm{K}_{V}$ 
current amplitude in mature pDA. Therefore, in agreement with studies performed in mammals, our present work identifies the presence of $\mathrm{O}_{2}$-sensitive $\mathrm{K}_{v}$ channels in the chicken DA. These data support the idea that the $\mathrm{K}_{v}$ channel inhibition in response to $\mathrm{O}_{2}$ is preserved during evolution, as recently suggested (31).

Inhibition of $\mathrm{K}_{\mathrm{v}}$ channels leads to membrane depolarization activation of $\mathrm{Ca}$ channels, increase in intracellular $\mathrm{Ca}^{2}$, and vasoconstriction $(12,22,24,32,38)$.

Thus $\mathrm{K}_{v}$ channel inhibition could be a key mechanism mediating $\mathrm{O}_{2}$-induced contraction in the chicken DA, as previously reported in mammals $(23,25,27$, 28). In agreement with this notion, the contractile response to $\mathrm{O}_{2}$ was partly inhibited by the CaL channel blocker nifedipine and nearly abolished in the absence of extracellular calcium (present work and Ref. 16). It is very likely that, apart from $\mathrm{Ca}$ L channel opening, $\mathrm{Ca}^{2}$ entry through store-operated channels (11, $16,17)$ or its release from intracellular stores (19) may also contribute to the normoxic contraction. Interestingly, compared with other chicken vascular beds (like the pulmonary arteries; unpublished observations) the DA appears to be less sensitive to dihydropyridines, as evidenced by the low potencies of BAY $\mathrm{K} 8644$ to induce contraction and of nifedipine to relax $\mathrm{KCl}$ constriction. This may explain the relatively high concentration of nifedipine $\left(10^{6} \mathrm{M}\right)$ needed to significantly reduce $\mathrm{O}_{2}$-induced contraction.

\subsubsection{Rho kinase as effector}

Activation of Rho kinase can phosphorylate the 130-kDa myosin binding subunit of myosin light chain phosphatase, causing its inhibition. This prevents myosin light chain dephosphorylation and hence increases $\mathrm{Ca}^{2}$ sensitivity and smooth muscle contractility. Thus Rho kinasemediated $\mathrm{Ca}^{2}$ sensitization is an important mechanism controlling vascular smooth muscle tone $(20,30)$. In the present study, we found that $\mathrm{O}_{2}$ constriction is diminished by $75 \%$ when extracellular $\mathrm{Ca}^{2}$ is removed. Similar results have been reported by Greyner and Dzialowski (16). The remaining $\mathrm{Ca}^{2}$-independent contraction is likely to be due to Rho kinaseinduced $\mathrm{Ca}^{2}$ sensitization, as reported in human and rabbit DA $(17,18)$. Consistent with this view, $\mathrm{O}_{2}$-induced constriction in mature pDA was relaxed by the Rho kinase inhibitors Y-27632 and hydroxyfasudil.

Maturation of $\mathrm{O}_{2}$-induced contraction. The mechanisms underlying the failure of the preterm DA to constrict and close after birth are not completely elucidated. In the chicken DA at 15 days of incubation, we have found (Ref. 1 and present study) that the response to $\mathrm{O}_{2}$ was very weak or absent. On the other hand, at 19-20 days of incubation all pDA were markedly constricted in response to $\mathrm{O}_{2}$. Our results are in agreement with previous studies in other species showing that 
the constriction of DA to $\mathrm{O}_{2}$ is developmentally regulated $(10,21,32)$. The reduced response to $\mathrm{O}_{2}$ in preterm DA may reflect a deficiency in the sensor, the mediator, the effector, or a combination of these. In this regard, we observed that $\mathrm{O}_{2}$ increased ROS content in mature but not in immature pDA. These differences are not related to changes in mRNA expression of SOD1 (which is even higher in the immature DA), SOD2, or catalase, and rather may reflect an impairment of the mitochondrial sensing mechanism in the immature pDA. In accordance with this, Kajimoto et al. (18) found that in response to $\mathrm{O}_{2}$, superoxide levels increased to higher levels in term than in preterm rabbit DASMC. Interestingly, we also observed that when the putative mediator (i.e., $\mathrm{H}_{2} \mathrm{O}_{2}$ ) was exogenously provided it induced contraction of the mature but not the immature pDA, suggesting that the unresponsiveness to $\mathrm{O}_{2}$ also involves immaturity of the final effectors.

Focusing on the putative effectors, we show that $\mathrm{K}_{v}$ currents are markedly reduced in immature versus mature pDA smooth muscle cells. In accordance with this, Thebaud et al. (32) observed that $\mathrm{K}_{v}$ current amplitudes in preterm rabbit DA are reduced compared with term DA. Among the different $\mathrm{K}_{\mathrm{v}}$ channels, $\mathrm{K}_{v} 1.5$ have been shown to be $\mathrm{O}_{2}$ sensitive and proposed as candidates to underlie $\mathrm{O}_{2}$-sensitive $\mathrm{K}_{v}$ currents in $\mathrm{DA}(23,32)$ and pulmonary arteries (5). However, the developmental increase in $K_{v}$ current found in the chicken DA seems to be unrelated to changes in $\mathrm{K}_{\mathrm{v}} 1.5$ channel expression, which even decreases with gestational age. In fact, contradictory data regarding the changes in $\mathrm{K}_{v} 1.5$ channels expression during the last days of gestation have been reported in different species $(32,41)$. One possibility is that other $\mathrm{O}_{2}$ sensitive $\mathrm{K}_{v}$ channels apart from $\mathrm{K}_{v} 1.5$ (such as $\mathrm{K}_{\mathrm{v}} 2.1$ ) make a major contribution to the total $\mathrm{K}_{v}$ current in chicken DA.

Very recently, Thebaud et al. (33) suggested that CaL channels are $\mathrm{O}_{2}$ sensitive in the rabbit DA and their functional immaturity contributes to the impaired response to $\mathrm{O}_{2}$ in the preterm animal. We found that nifedipine, a CaL channel blocker, was more potent to inhibit $\mathrm{KCl}$-induced contractions in the mature than the immature pDA. In addition, the CaL channel activator BAY K8644 was also much more effective in inducing a contraction in the mature than in the immature pDA. Nifedipine and BAY K8644 belong to the same chemical class of dihydropyridines and bind to the same site in the channel (15). Thus our data indicate a maturational change in the response to dihydropyridines. In the study of Thebaud et al. (33), the presence of BAY K8644 enhanced the $\mathrm{O}_{2}$ constriction in preterm (to levels observed at term) but not in term DA. On the contrary, we found that in the preterm chicken DA the immaturity cannot be reversed by BAY K8644. 
Finally, the reduced response to $\mathrm{O}_{2}$ in preterm compared with term DA has also been associated with a diminished expression/activity of components of the Rho-Rho kinase pathway $(11,18)$. Consistent with this, we have observed that the sensitivity to the Rho kinase inhibitors hydroxyfasudil and Y-27632 increased with development.

\subsubsection{Changes in response to $\mathrm{O}_{2}$ along the $D A$}

One of the most striking features of the chicken DA is the radically opposite response to changes in $\mathrm{O}_{2}$ (relaxation vs. contraction) seen in the two portions (aortic vs. pulmonary) of the vessel. This functional difference correlates with morphological changes along the chicken DA between the pulmonary artery and the aorta $(1,7,8)$, which may reflect the proposed different embryological origin of the cells composing the aortic and the pulmonary portions of the vessel (8). Here we have examined the mechanisms involved in the different responses to $\mathrm{O}_{2}$ in both sides of the vessel. The major finding was that $\mathrm{O}_{2}$ did not increase ROS content in mature aDA. This may be explained, at least partly, by a higher expression of the $\mathrm{H}_{2} \mathrm{O}_{2}$ scavenging enzyme catalase. Nevertheless, this does not seem to be the only difference along the vessel. Thus, unlike the pulmonary side, the aortic side of the DA relaxed in response to the $\mathrm{H}_{2} \mathrm{O}_{2}$ analog (the proposed mediator). This opposite behavior cannot be explained by a different expression/activity of the effector proteins (i.e., Kv channels and Rho kinase) in both sides, since the inhibition of $K_{v}$ channels caused a similar contraction in both sides of the vessel and, conversely, the Rho kinase inhibitors relaxed both the aortic and the pulmonary ductal segments.

\subsection{Conclusions}

In summary, our results offer supportive evidence implicating the mitochondrial ETC as sensor, $\mathrm{H}_{2} \mathrm{O}_{2}$ as mediator, and $\mathrm{K}_{v}$ channels and Rho kinase as effectors of $\mathrm{O}_{2}$-induced contraction in the chicken DA (Fig. 2.10). Therefore, there is a conserved mechanism for $\mathrm{O}_{2}$ sensing/signaling in mammalian and nonmammalian DA. Our results also indicate that the developmental response to $\mathrm{O}_{2}$ involves a parallel maturation of the three components: sensor, mediator, and effectors. 


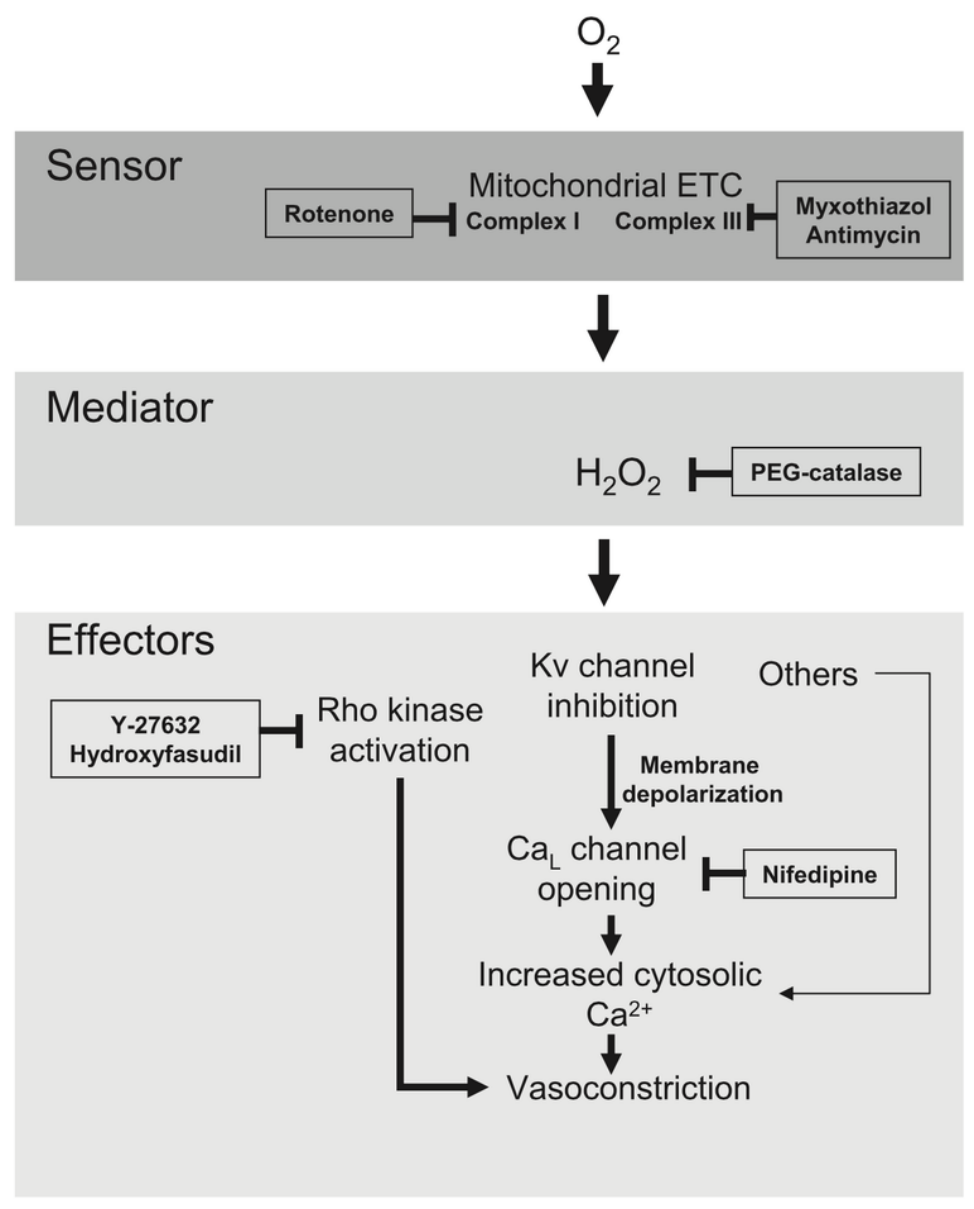

Fig. 2.10. Mechanism proposed by Michelakis et al. for normoxic constriction of the mammalian DA (mitochondrial ETC as sensor, $\mathrm{H}_{2} \mathrm{O}_{2}$ as mediator, and $\mathrm{K}_{\mathrm{V}}$ channels and Rho kinase as effectors) is also responsible for normoxic constriction of chicken DA. Scheme also shows the inhibitors used targeting different steps of the pathway. 


\section{References}

1. Agren P, Cogolludo AL, Kessels CG, Perez-Vizcaino F, De Mey JG, Blanco CE, Villamor E. Ontogeny of chicken ductus arteriosus response to oxygen and vasoconstrictors. Am J Physiol Regul Integr Comp Physiol 292: R485-R496, 2007.

2. Agren $P$, van der Sterren S, Cogolludo AL, Blanco CE, Villamor E. Developmental changes in the effects of prostaglandin $E_{2}$ in the chicken ductus arteriosus. J Comp Physiol [B] 179: 133-143, 2009.

3. Agren P, van der Sterren S, Cogolludo AL, Frazziano G, de Mey JG, Blanco CE, Villamor E. Developmental changes in endothelium-dependent relaxation of the chicken ductus arteriosus. J Physiol Pharmacol 59: 55-76, 2008.

4. Archer SL, Michelakis ED, Thebaud B, Bonnet S, Moudgil R, Wu XC, Weir EK. A central role for oxygen-sensitive $\mathrm{K}$ channels and mitochondria in the specialized oxygen-sensing system. Novartis Found Symp 272: 157-175, 214-217, 2006.

5. Archer SL, Souil E, Dinh-Xuan AT, Schremmer B, Mercier JC, El Yaagoubi A, Nguyen-Huu L, Reeve HL, Hampl V. Molecular identification of the role of voltagegated $\mathrm{K}$ channels, Kv1.5 and Kv21, in hypoxic pulmonary vasoconstriction and control of resting membrane potential in rat pulmonary artery myocytes. J Clin Invest 101: 2319-2330, 1998.

6. Belanger C, Copeland J, Muirhead D, Heinz D, Dzialowski EM. Morphological changes in the chicken ductus arteriosi during closure at hatching. Anat Rec (Hoboken) 291: 1007-1015, 2008.

7. Bergwerff M, DeRuiter MC, Gittenberger-de Groot AC. Comparative anatomy and ontogeny of the ductus arteriosus, a vascular outsider. Anat Embryol (Berl) 200: 559-571, 1999.

8. Bergwerff M, DeRuiter MC, Poelmann RE, Gittenberger-de Groot AC. Onset of elastogenesis and downregulation of smooth muscle actin as distinguishing phenomena in artery differentiation in the chick embryo. Anat Embryol (Berl) 194: 545-557, 1996.

9. Clyman RI. Mechanisms regulating the ductus arteriosus. Biol Neonate 89: 330335, 2006.

10. Clyman RI, Mauray F, Wong L, Heymann MA, Rudolph AM. The developmental response of the ductus arteriosus to oxygen. Biol Neonate 34: 177-181, 1978.

11. Clyman RI, Waleh N, Kajino H, Roman C, Mauray F. Calciumdependent and calcium-sensitizing pathways in the mature and immature ductus arteriosus. Am J Physiol Regul Integr Comp Physiol 293: R1650- R1656, 2007.

12. Cogolludo A, Moreno L, Bosca L, Tamargo J, Perez-Vizcaino F. Thromboxane A2induced inhibition of voltage-gated $\mathrm{K}$ channels and pulmonary vasoconstriction: role of protein kinase Czeta. Circ Res 93: 656-663, 2003.

13. Cogolludo A, Moreno L, Lodi F, Frazziano G, Cobeno L, Tamargo J, Perez-Vizcaino F. Serotonin inhibits voltage-gated K currents in pulmonary artery smooth muscle 
cells: role of $5-\mathrm{HT} 2 \mathrm{~A}$ receptors, caveolin-1, and KV15 channel internalization. Circ Res 98: 931-938, 2006.

14. Chandel NS, Schumacker PT. Cellular oxygen sensing by mitochondria: old questions, new insight. J Appl Physiol 88: 1880-1889, 2000.

15. Godfraind T, Miller R, Wibo M. Calcium antagonism and calcium entry blockade. Pharmacol Rev 38: 321-416, 1986.

16. Greyner H, Dzialowski EM. Mechanisms mediating the oxygen-induced vasoreactivity of the ductus arteriosus in the chicken embryo. Am J Physiol Regul Integr Comp Physiol 295: R1647-R1659, 2008.

17. Hong Z, Hong F, Olschewski A, Cabrera JA, Varghese A, Nelson DP, Weir EK. Role of store-operated calcium channels and calcium sensitization in normoxic contraction of the ductus arteriosus. Circulation 114: 1372-1379, 2006.

18. Kajimoto H, Hashimoto K, Bonnet SN, Haromy A, Harry G, Moudgil R, Nakanishi T, Rebeyka I, Thebaud B, Michelakis ED, Archer SL. Oxygen activates the Rho/Rhokinase pathway and induces RhoB and ROCK-1 expression in human and rabbit ductus arteriosus by increasing mitochondria-derived reactive oxygen species: a newly recognized mechanism for sustaining ductal constriction. Circulation 115: 1777-1788, 2007.

19. Keck M, Resnik E, Linden B, Anderson F, Sukovich DJ, Herron J, Cornfield DN. Oxygen increases ductus arteriosus smooth muscle cytosolic calcium via release of calcium from inositol triphosphate-sensitive stores. Am J Physiol Lung Cell Mol Physiol 288: L917-L923, 2005.

20. Loirand G, Guerin P, Pacaud P. Rho kinases in cardiovascular physiology and pathophysiology. Circ Res 98: 322-334, 2006.

21. McMurphy DM, Heymann MA, Rudolph AM, Melmon KL. Developmental changes in constriction of the ductus arteriosus: responses to oxygen and vasoactive agents in the isolated ductus arteriosus of the fetal lamb. Pediatr Res 6: 231-238, 1972.

22. Michelakis E, Rebeyka I, Bateson J, Olley P, Puttagunta L, Archer S. Voltage-gated potassium channels in human ductus arteriosus. Lancet 356: 134-137, 2000.

23. Michelakis ED, Rebeyka I, Wu X, Nsair A, Thebaud B, Hashimoto K, Dyck JR, Haromy A, Harry G, Barr A, Archer SL. $\mathrm{O}_{2}$ sensing in the human ductus arteriosus: regulation of voltage-gated $\mathrm{K}$ channels in smooth muscle cells by a mitochondrial redox sensor. Circ Res 91: 478-486, 2002.

24. Nelson MT, Quayle JM. Physiological roles and properties of potassium channels in arterial smooth muscle. Am J Physiol Cell Physiol 268: C799-C822, 1995.

25. Olschewski A, Hong Z, Peterson DA, Nelson DP, Porter VA, Weir EK. Opposite effects of redox status on membrane potential, cytosolic calcium, and tone in pulmonary arteries and ductus arteriosus. Am J Physiol Lung Cell Mol Physiol 286: L15-L22, 2004.

26. Platoshyn O, Brevnova EE, Burg ED, Yu Y, Remillard CV, Yuan JX. Acute hypoxia selectively inhibits KCNA5 channels in pulmonary artery smooth muscle cells. Am J Physiol Cell Physiol 290: C907-C916, 2006. 
27. Reeve HL, Tolarova S, Nelson DP, Archer S, Weir EK. Redox control of oxygen sensing in the rabbit ductus arteriosus. J Physiol 533: 253-261, 2001.

28. Reeve HL, Weir EK, Nelson DP, Peterson DA, Archer SL. Opposing effects of oxidants and antioxidants on $\mathrm{K}$ channel activity and tone in rat vascular tissue. Exp Physiol 80: 825-834, 1995.

29. Smith GC. The pharmacology of the ductus arteriosus. Pharmacol Rev 50: 35-58, 1998.

30. Somlyo AP, Somlyo AV. $\mathrm{Ca}^{2}$ sensitivity of smooth muscle and nonmuscle myosin II: modulated by $\mathrm{G}$ proteins, kinases, and myosin phosphatase. Physiol Rev 83: 13251358, 2003.

31. Sutendra G, Michelakis ED. The chicken embryo as a model for ductus arteriosus developmental biology: cracking into new territory. Am J Physiol Regul Integr Comp Physiol 292: R481-R484, 2007.

32. Thebaud B, Michelakis ED, Wu XC, Moudgil R, Kuzyk M, Dyck JR, Harry G, Hashimoto K, Haromy A, Rebeyka I, Archer SL. Oxygensensitive Kv channel gene transfer confers oxygen responsiveness to preterm rabbit and remodeled human ductus arteriosus: implications for infants with patent ductus arteriosus. Circulation 110: 1372-1379, 2004.

33. Thebaud B, Wu XC, Kajimoto H, Bonnet S, Hashimoto K, Michelakis ED, Archer SL. Developmental absence of the $\mathrm{O}_{2}$ sensitivity of L-type calcium channels in preterm ductus arteriosus smooth muscle cells impairs $\mathrm{O}_{2}$ constriction contributing to patent ductus arteriosus. Pediatr Res 63: 176-181, 2008.

34. Tristani-Firouzi M, Reeve HL, Tolarova S, Weir EK, Archer SL. Oxygen-induced constriction of rabbit ductus arteriosus occurs via inhibition of a 4-aminopyridine-, voltage-sensitive potassium channel. J Clin Invest 98: 1959-1965, 1996.

35. van der Sterren S, Agren P, Zoer B, Kessels L, Blanco CE, Villamor E. Morphological and functional alterations of the ductus arteriosus in a chicken model of hypoxiainduced fetal growth retardation. Pediatr Res 65: 279-284, 2009.

36. Villamor E, Ruijtenbeek K, Pulgar V, De Mey JG, Blanco CE. Vascular reactivity in intrapulmonary arteries of chicken embryos during transition to ex ovo life. Am $J$ Physiol Regul Integr Comp Physiol 282: R917-R927, 2002.

37. Ward JP. Oxygen sensors in context. Biochim Biophys Acta 1777: 1-14, 2008.

38. Weir EK, Lopez-Barneo J, Buckler KJ, Archer SL. Acute oxygensensing mechanisms. N Engl J Med 353: 2042-2055, 2005.

39. Weir EK, Reeve HL, Cornfield DN, Tristani-Firouzi M, Peterson DA, Archer SL. Diversity of response in vascular smooth muscle cells to changes in oxygen tension. Kidney Int 51: 462-466, 1997.

40. Wolin MS, Ahmad M, Gupte SA. Oxidant and redox signaling in vascular oxygen sensing mechanisms: basic concepts, current controversies, and potential importance of cytosolic NADPH. Am J Physiol Lung Cell Mol Physiol 289: L159L173, 2005. 
41. Wu C, Hayama E, Imamura S, Matsuoka R, Nakanishi T. Developmental changes in the expression of voltage-gated potassium channels in the ductus arteriosus of the fetal rat. Heart Vessels 22: 34-40, 2007. 
Chapter 3

\section{Morphological and functional alterations of the ductus arteriosus in a chicken model of hypoxia-induced fetal growth retardation}

Saskia van der Sterren, Pia Agren, Bea Zoer, Lilian Kessels, Carlos E. Blanco, and Eduardo Villamor Pediatric Research Vol. 65, No. 3, 2009 


\begin{abstract}
The hypoxic conditions in which children with intrauterine growth retardation (IUGR) develop are hypothesized to alter the development of the ductus arteriosus (DA). We aimed to evaluate the effects of in ovo hypoxia on chicken DA morphometry and reactivity. Hypoxia (15\% O2 from day 6 to 19 of the 21-d incubation period) produced a reduction in the body mass of the 19-d fetuses and a shortening of right and left DAs. However, ductal lumen and media crosssectional areas were not affected by hypoxia. The ductal contractions induced by oxygen, $\mathrm{KCl}, \mathrm{H}_{2} \mathrm{O}_{2}$, 4-aminopyridine, and endothelin-1 were similar in control and hypoxic fetuses. In contrast, the DAs from the hypoxic fetuses showed increased contractile responses to norepinephrine and phenylephrine and impaired relaxations to acetylcholine, sodium nitroprusside, and isoproterenol. The relaxations induced by 8-Br-cGMP, forskolin, Y-27632, and hydroxyfasudil were not altered by chronic hypoxia. In conclusion, chronic in ovo hypoxiainduced growth retardation in fetal chickens and altered the response of the DA to adrenergic agonists and to endothelium-dependent and -independent relaxing agents. Our observations support the concept that prolonged patency of the DA in infants with IUGR may be partially related with hypoxia-induced changes in local vascular mechanisms.
\end{abstract}




\subsection{Introduction}

Intrauterine growth retardation (IUGR) is most frequently the consequence of placental insufficiency resulting in decreased availability of nutrients and oxygen (1). Although IUGR is considered as an important cause of pre- and post- natal morbidity, its impact on the several areas of morbidity associated with prematurity remains controversial. Reports vary from higher to similar rates of respiratory distress syndrome, chronic lung disease, necrotizing enterocolitis, retinopathy of prematurity, and patent ductus arteriosus (DA) in preterm infants with and without IUGR $(1,2)$.

The putative alterations produced by hypoxia and other prenatal insults in the normal development of DA are unclear. Histologic evidence of accelerated maturation of the DA was described in the autopsy of preterm infants exposed to chronic intrauterine stress, leading to the hypothesis that this may have resulted clinically in effective postnatal DA closure (3). In contrast, other authors have described in the DA of premature infants with IUGR, histologic changes, such as fragmentation, coagulation, necrosis of the internal elastic lamina, hemorrhage with necrosis, and loosening of elastic fibers and muscles in the tunica media that may have produced subsequent patent DA (4). The chicken fetus is an excellent model for the study of the cardiovascular consequences of prenatal growth retardation (5-7). Recently, we have studied the chicken DA reactivity and demonstrated that the effects of vasoactive mediators on its tone are developmentally regulated with loss of responsive-ness to vasodilators and increase of responsiveness to vasoconstrictors with advancing age (8-10).

Hypoxia has profound effects on endothelial and vascular smooth muscle cellular physiology affecting the transcriptionally regulated expression of vasoactive substances, the modulation of receptor populations, the density and activities of ion channels, and the signal transduction pathways involved in modulating vascular tone (5-7,11-14). Postnatal constriction of the full-term DA produces hypoxia of the muscle media, and this stimulus appears as essential for the anatomic remodeling that prevents subsequent ductal reopening (15). In the present study, we hypothesize that prolonged hypoxic exposure alters the development of the DA contractile apparatus that is essential for rapid constriction after birth. Therefore, our aim was to evaluate the effects of in ovo hypoxia on the chicken DA reactivity and morphology. 


\subsection{Methods}

\subsection{1 incubation and hypoxia protocol}

Experiments were performed in accordance with Dutch law for animal experimentation and were approved by the Ethical Committee of the University of Maastricht. Fertile Lohman-selected White Leghorn eggs were incubated at $37.8^{\circ} \mathrm{C}$ and $45 \%$ humidity (Incubator model $25 \mathrm{HS}$, Masalles Comercial, Spain). Control embryos were incubated under normoxic conditions $\left(21 \% \mathrm{O}_{2} \quad 0.03 \%\right.$ $\mathrm{CO}_{2}$ ). Experimental embryos were incubated under normoxic conditions until day 6 of incubation when eggs were transferred to a second incubator where hypoxia ( $15.0 \pm 0.3 \%$ atmospheric $\mathrm{O}_{2}, 0.03 \% \mathrm{CO}_{2}$ ) was maintained by providing a constant flow of $\mathrm{N}_{2}$ and compressed air with a flow meter. The $\mathrm{O}_{2}$ and $\mathrm{CO}_{2}$ concentrations in the incubator were monitored with a DrDAQ $\mathrm{O}_{2}$ sensor (Pico Technology, United Kingdom) and an infrared $\mathrm{CO}_{2}$ analyzer (Beckman Instruments, Inc., Fullerton, CA).
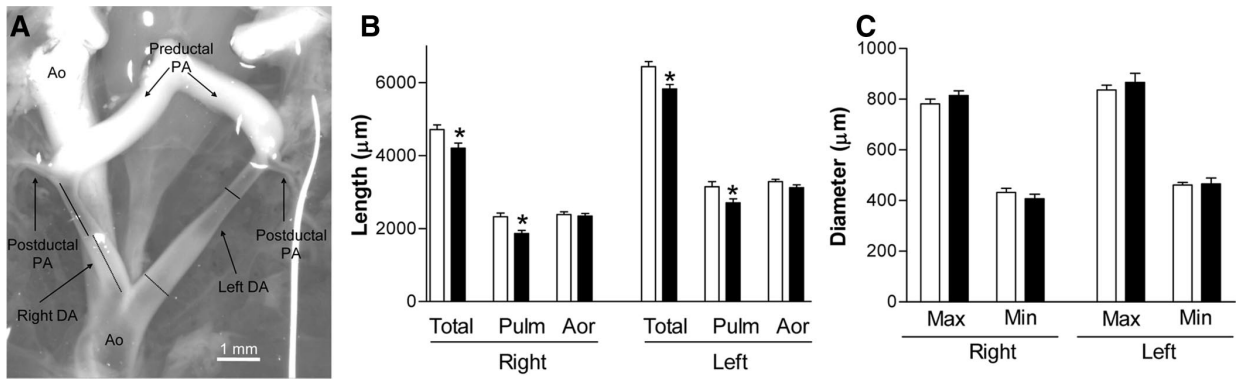

Fig. 3.1. Anatomy of the chicken DA. The method used to determine the length and diameter of the pulmonary (solid line) and the aortic (dashed line) of the DAs is also depicted. Ao, aorta; PA, pulmonary artery. Lengths $(B)$ and diameters $(C)$ of the DAs of 19-d chicken fetuses exposed to hypoxia (•) or normoxia() during incubation. Pulm, pulmonary side; Aor, aortic side. Each bar represents the mean \pm SEM of eight animals. ${ }^{*} p<0.05$ for difference from normoxia.

\subsubsection{Morphometric analysis of the $D A$}

On day 19 of incubation, the embryos were taken out and immediately killed by decapitation. With the aid of a dissecting microscope (Mild M8; Leica, Cambridge, United Kingdom), the heart, the esophagus and the crop were removed, the right and the left DA were exposed, and the preparation was photographed (Fig. 3.1) with a digital camera (Leica DFC 280). Pictures were subsequently analyzed with a computerized morphometric system (Quantimet 
A
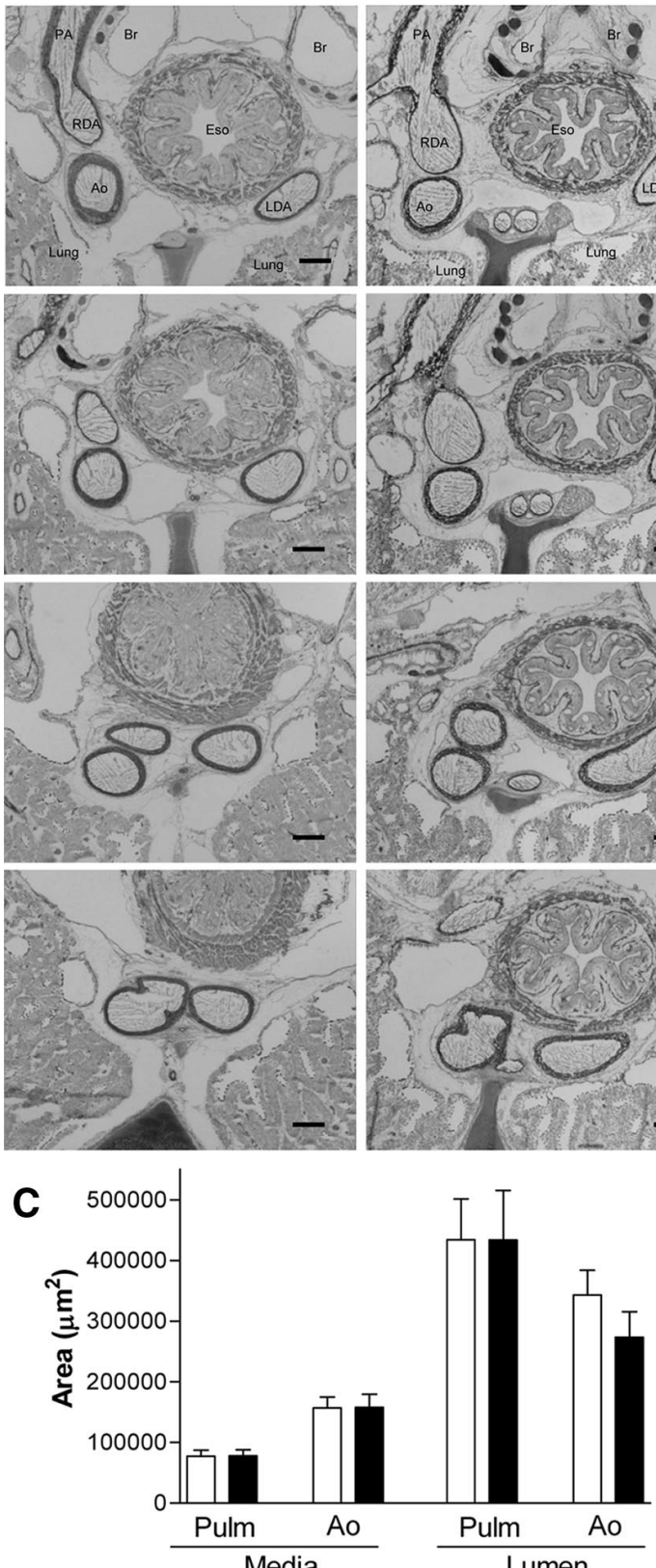

B
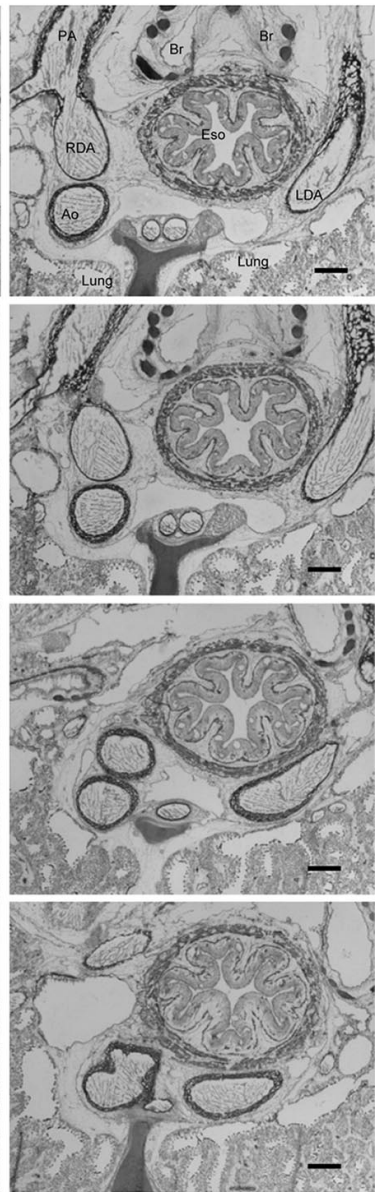

Lumen

Figure 3.2. Histology of the chicken DA. Light micrographs showing double staining for elastin and a-actin of cross-sections of snap-frozen 19-d chicken fetuses exposed to normoxia $(A)$ or hypoxia (B) during incubation. Bars $200 \mu \mathrm{M}$. Ao, aorta; PA, pulmonary artery; Eso, esophagus; Br, bronchus; $\mathrm{R}$, right; $\mathrm{L}$, left. $(C)$ The media and lumen cross-sectional areas of the right DA were not significantly different between normoxic $(\square)$ and hypoxic ( $\bullet$ ) fetuses. Each bar represents the mean \pm SEM of five animals. 
570C; Leica,). Analysis was performed by a single observer (P.A.), who was unaware of the experimental conditions. The total length of the DAs, the length of the aortic and the pulmonary segments, and the maximal and minimal diameters were deter- mined. Each measurement was repeated three times and the mean value recorded.

Microscopic morphometric analysis was assessed in DAs fixed in situ with the whole-body freezing technique. Embryos were frozen in $1 \%$ carboxymethylcellulose in liquid nitrogen. Serial, transversal sections (8 $\mu \mathrm{m}$ thick) through the whole length of the DAs, were obtained on a Leica freezing microtome (CM 3050S) and double staining was performed for elastin (Lawson solution; Klinipath, Duiven, The Netherlands) and a MAb against smooth muscle a-actin (Dako, Carpinteria, CA). Microscopic images were analyzed with the Quantimet $570 \mathrm{C}$ to determine media layer cross-sectional area (CSA), media wall thickness, and intraluminal CSA. This analysis was only performed in the right DA because its orientation allowed the obtention of transversal sections of the vessel (Fig. 3.2). From each specimen, 3-5 sections with the morphology of the pulmonary side and 8-10 with the morphology of the aortic side of the DA were analyzed. Sections with a transitional morphology between the two parts were discarded for analysis. Analysis was performed by a single observer (L.K.), who was unaware of the experimental conditions.

\subsubsection{Recording of DA reactivity}

Reactivity was analyzed only in the proximal (pulmonary) side of the vessel. Rings from hypoxic and normoxic embryos were studied in parallel. The myograph organ bath was filled with Krebs-Ringer bicarbonate (KRB) buffer maintained at $39^{\circ} \mathrm{C}$ and continuously aerated with one of the following gas mixtures: $95 \% \mathrm{~N}_{2} / 5 \% \mathrm{CO}_{2}\left(\mathrm{PO}_{2}=2.6-3.3 \mathrm{kPa}\right), 5 \% \mathrm{O}_{2} / 90 \% \mathrm{~N}_{2} / 5 \% \mathrm{CO}_{2}\left(\mathrm{PO}_{2}=\right.$ $6.8-7.2 \mathrm{kPa})$, or $21 \% \mathrm{O}_{2} / 74 \% \mathrm{~N}_{2} / 5 \% \mathrm{CO}_{2}\left(\mathrm{PO}_{2}=16-20 \mathrm{kPa}\right)$. The final $\mathrm{pH}$ was 7.38-7.42 and $\mathrm{PCO}_{2}$ was $4.6-5.6 \mathrm{kPa}$ in all solutions (8). Each DA ring was stretched to its individual optimal lumen diameter, i.e., the diameter at which it developed the strongest contractile response to $62.5 \mathrm{mM} \mathrm{KCl}$ (8). During the first phase of stabilization and determination of optimal diameter, KRB buffer was aerated with $95 \% \mathrm{~N}_{2} / 5 \% \mathrm{CO}_{2}$. Afterward, and depending on the specific protocol, one of the above-described gas mixtures was used.

To assess the responsiveness of the DA to oxygen, vessels were incubated for 30 min with $95 \% \mathrm{~N}_{2} / 5 \% \mathrm{CO}_{2}$. Then the gas mixture was switched to $21 \%$ $\mathrm{O}_{2} / 74 \% \mathrm{~N}_{2} / 5 \%$ CO2. This gas was maintained for $10 \mathrm{~min}$. In another set of experiments, the organ chamber was bubbled with $5 \% \mathrm{O}_{2} / 90 \% \mathrm{~N}_{2} / 5 \% \mathrm{CO}_{2}$ and concentration-response curves to $\mathrm{KCl}$, the inhibitor of voltage-gated $(\mathrm{Kv}) \mathrm{K}^{+}$ 
channels 4-aminopyridine (4-AP), $\mathrm{H}_{2} \mathrm{O}_{2}$, endothelin-1 (ET-1), norepinephrine $(\mathrm{NE})$, and phenylephrine (Phe) were constructed. Relaxing agonists were evaluated during contraction induced by $62.5 \mathrm{mM} \mathrm{K}$. Concentration-response curves for acetylcholine (ACh), the nitric oxide (NO) donor sodium nitroprusside (SNP), the cyclic guanosine monophosphate (cGMP) analog 8-Br-cGMP, the $\beta$ adrenoceptor agonist isoproterenol, the adenylyl cyclase activator forskolin, and the Rho-kinase inhibitors Y-27632 and hydroxyfasudil were constructed.

\subsubsection{Drugs and solutions}

KRB contained (in mmol/L): $\mathrm{NaCl}, 118.5 ; \mathrm{KCl}, 4.75 ; \mathrm{MgSO}_{4} \cdot 7 \mathrm{H}_{2} \mathrm{O}, 1.2 ; \mathrm{KH}_{2} \mathrm{PO}_{4}$, 1.2; $\mathrm{NaHCO}_{3}, 25.0 ; \mathrm{CaCl}_{2}$, 2.5; glucose, 5.5. Solutions containing different concentrations of $\mathrm{K}^{+}$were prepared by replacing part of the $\mathrm{NaCl}$ by an equimolar amount of $\mathrm{KCl}$. All the drugs were obtained from Sigma Chemical Co (St. Louis, MO) and dissolved initially in distilled deionized water (except forskolin in ethanol).

\subsubsection{Data analysis}

Results are shown as mean (SD) of measurements in $n$ animals. For clarity, results in the figures are shown as mean \pm SEM. Contractions are expressed in Newton/meter and relaxations as percentage of reduction of the contraction induced by $\mathrm{K}^{+}$. Sensitivity (expressed as $\mathrm{pD}_{2}=-\log \mathrm{EC}_{50}$ ) and maximal contraction or relaxation $\left(E_{\max }\right)$ to agonists was determined by fitting individual concentration-response data to a nonlinear sigmoidal regression curve. Comparisons were made by an unpaired $t$ test. Differences were considered significant at a $p<0.05$.

\subsection{Results}

\subsubsection{Fetal growth and DA morphometry}

Exposure of chicken fetuses to $15 \%$ instead of $21 \% \mathrm{O}_{2}$ from day 6 to day 19 of incubation induced growth retardation (body mass normoxic: $29.9 \mathrm{~g}, \mathrm{SD} 5.6, n=$ 34; hypoxic: 25.3 , SD 5.5, $n=32, p<0.01$ versus normoxic).

Both the right and the left DAs were shorter in the hypoxic fetuses (Fig. 3.1B). When the lengths of pulmonary and the aortic sides of the DA were analyzed, it was observed that the ductal shortening induced by hypoxia was due to a shortening of the pulmonary segment (Fig. 3.1B). The external diameter of the DA was not significantly different between normoxic and hypoxic fetuses (Fig. 3.1C). 
As shown in Fig. 3.2, the chicken DAs exhibited two distinct phenotypes along their lengths. The proximal (pulmonary) DA displayed the structure of a muscular artery with a dense a-actin-positive media subjacent to the endothelium and few layers of elastic fibers around the muscular layer. The distal (aortic) DA contains many elastic fibers that fill the vascular wall arranged in concentric lamellae with a-actin-positive smooth muscle cells embedded between the lamellae. The muscular phenotype was present in $49.4 \%$ (SD 8.7, $n=5$ ) of the length of the right DA from the normoxic animals and in $33.0 \%$ (SD $11.5, n=5, p<0.05$ versus control) of the vessel length from the hypoxic group. The lumen CSA was significantly greater in the pulmonary than the aortic side of the DA and the media CSA was significantly greater in the aortic than in the pulmonary side of the vessel. The media and lumen CSAs of the right DA were not significantly different between normoxic and hypoxic fetuses (Fig. 3.2C).

\subsection{2 $D$ A reactivity}

Switching the $\mathrm{O}_{2}$ mixture from 0 to $21 \%$ ( 3 to $18 \mathrm{kPa}$ ) produced a progressive increase of the $\mathrm{O}_{2}$ concentration in the organ chamber that reached a steady state after $\sim 3 \mathrm{~min}$ (8). This increase in $\mathrm{O}_{2}$ concentration resulted in a contractile response of the DA that was not significantly different between hypoxic and normoxic fetuses (Fig. 3.3A). In addition, the contractions induced by 4-AP (Fig. $3.3 A$ ), $\mathrm{KCl}$ (Fig. $3 B ; \mathrm{pD}_{2}$ normoxic: $1.61, \mathrm{SD} 0.12$; hypoxic: 1.69 , SD $0.14 ; p>$ 0.05), $\mathrm{H}_{2} \mathrm{O}_{2}$ (Fig. $3 C$ ), and ET-1 (Fig. 3.3D) did not vary between the experimental groups. The diameter at which maximal responses to depolarizing high- $\mathrm{K}^{+}$solution were obtained was significantly higher in the DAs from the hypoxic fetuses (843.1 $\mu \mathrm{m}, \mathrm{SD} 92.31, n=32$ versus $775.8 \mu \mathrm{m}, \mathrm{SD} 92.3, n=32$; $p<0.05$ ), but the passive wall stretch induced by this optimal diameter was not significantly different (normoxia: $0.89 \mathrm{~N} / \mathrm{m}$, SD 0.19; hypoxia: $0.92 \mathrm{~N} / \mathrm{m}$, SD 0.24).

The adrenergic agonists NE (Fig. 3.3E) and Phe (Fig. 3.3F) induced significantly $(p<0.05)$ larger contractions in the DAs from the hypoxic (NE: $0.91 \mathrm{~N} / \mathrm{m}, \mathrm{SD}$ $0.22, n=11$; Phe:0.87 N/m, SD 0.20, $n=11$ ) than in those from the normoxic fetuses (NE: $0.72 \mathrm{~N} / \mathrm{m}$, SD 0.23, $n=11$; Phe:0.69 N/m, SD 0.18, $n=11$ ). However, the sensitivity $\left(\mathrm{pD}_{2}\right)$ to $\mathrm{NE}$ (normoxic: 6.67, SD 0.22; hypoxic: 6.65, SD 0.23) and Phe (normoxic: 5.42, SD 0.15; hypoxic: 5.45, SD 0.17) did not vary between the experimental groups.

Acetylcholine (Fig. 3.4A), SNP (Fig. 3.4B), 8-Br-cGMP (Fig. 3.4C), isoproterenol (Fig. 3.5A), forskolin (Fig. 3.5B), Y-27632 (Fig. 3.5C), and hydroxyfasudil (Fig. $3.5 \mathrm{D}$ ) relaxed DA rings (precontracted with $62.5 \mathrm{mM} \mathrm{KCl}$ ) in a concentrationdependent manner. The relaxations induced by ACh ( $E_{\max }$ normoxic: $39.49 \%$, 
SD 16.2, $n=8$; $E_{\max }$ hypoxic: $15.02 \%$, SD 10.2, $n=8, p<0.05$ versus normoxic), SNP ( $E_{\max }$ normoxic: $59.9 \%$, SD 27.7, $n=8$; $E_{\max }$ hypoxic: $33.1 \%$, SD 16.4, $n=8, p<0.05$ versus normoxic), and isoproterenol ( $E_{\max }$ normoxic: $168.1 \%$, SD 19.4, $n=8$; $E_{\max }$ hypoxic: $112 \%$, SD 16.4, $n=8, p<0.05$ versus normoxic) were significantly impaired in the DAs from the fetuses incubated under hypoxic conditions. The sensitivity to SNP was significantly greater in normoxic than in hypoxic fetuses ( $\mathrm{pD}_{2}$ normoxic: $5.84, \mathrm{SD} 0.4 ; \mathrm{pD}_{2}$ hypoxic 4.95, SD $0.41, p<0.01$ versus normoxic). The sensitivities to $A C h$ and isoproteronol did not differ significantly between groups. The relaxations induced by 8-Br-cGMP, forskolin, Y-27632, and hydroxyfasudil were not significantly different when normoxic and hypoxic DAs were compared.
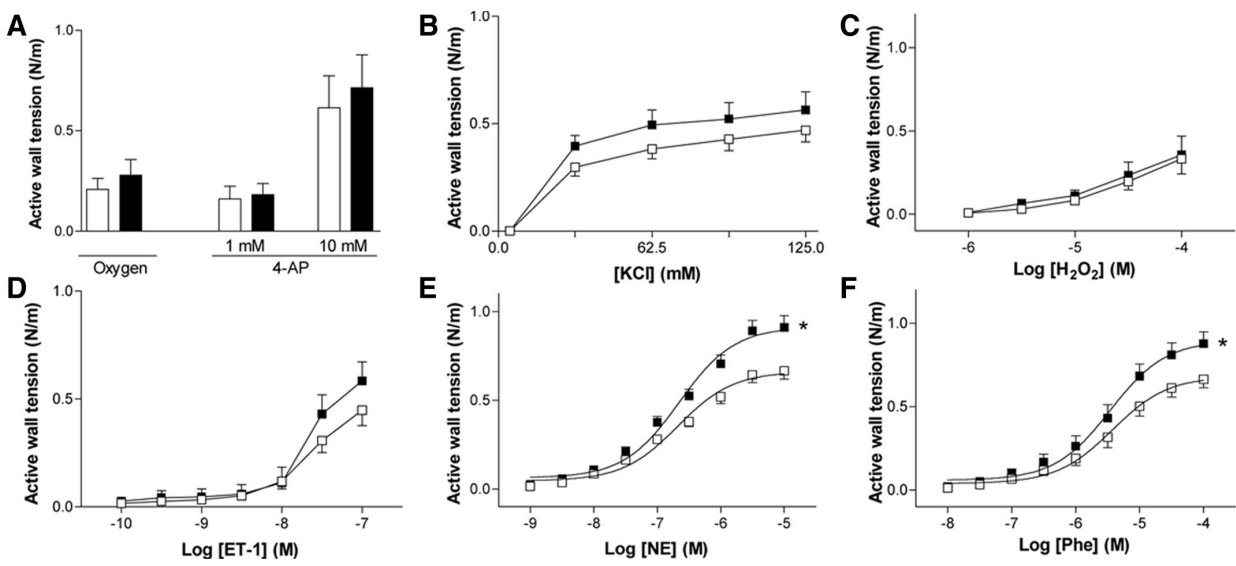

Fig. 3.3. Contractile effects of oxygen, 4-AP, $\mathrm{KCl}, \mathrm{H}_{2} \mathrm{O} 2$, ET-1, norepinephrine, and phenylephrine in DA rings of 19-d chicken fetuses exposed to hypoxia (-) or normoxia (ㅁ) during incubation. Each point (or bar) represents the mean \pm SEM of $8-11$ animals. ${ }^{*} p<0.05$ for difference in $E_{\max }$ from normoxia.
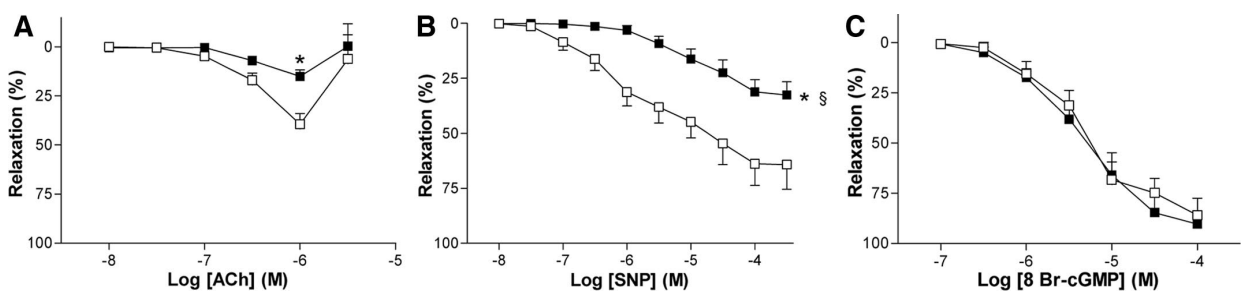

Fig. 3.4. Concentration-dependent relaxant effects of acetylcholine, SNP, and 8-Br cGMP in DA rings of 19-d chicken fetuses incubated under hypoxia ( $\bullet$ ) or normoxia ( $\square$ ). Each point represents the mean \pm SEM of $6-11$ animals. ${ }^{*} p<0.05$ for difference in $E_{\max }$ from normoxia. $\$ p<0.05$ for difference in $\mathrm{pD}_{2}$ from normoxia. 

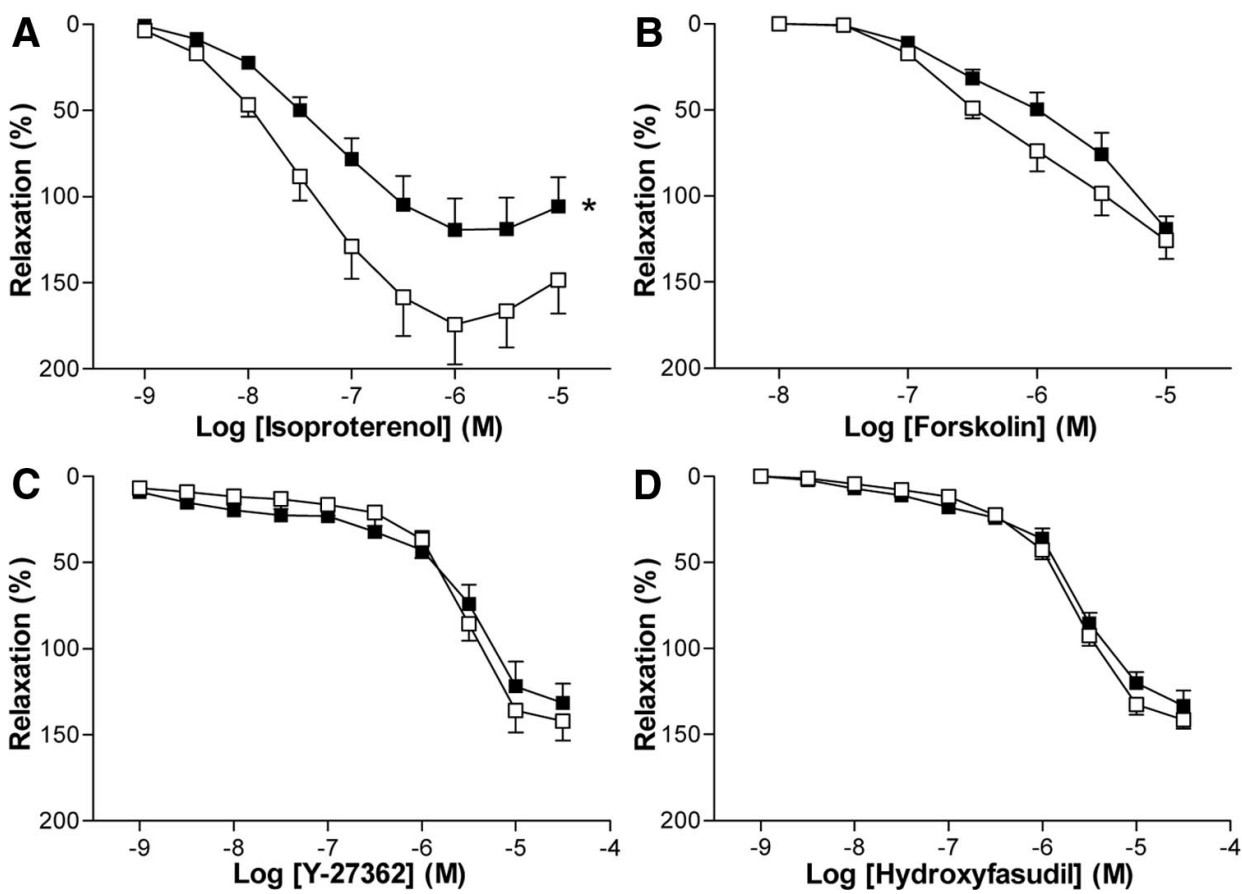

Fig. 3.5. Concentration-dependent relaxant effects of isoproterenol, forskolin, Y-27632, and hydroxyfasudil in DA rings of 19-d chicken fetuses incubated under hypoxia (•) or normoxia (口). Each point represents the mean + SEM of $6-11$ animals. ${ }^{*} p<0.05$ for difference in $E_{\max }$ from normoxia.

\subsection{Discussion}

We have characterized the morphologic and functional status of the DA in a chicken model of hypoxia-induced fetal growth retardation (5-7). We did not find significant evidences for an accelerated or delayed anatomical remodeling of the DA in the growth-retarded chicken fetuses. In contrast, the DA of the hypoxic fetuses showed an enhanced contractile response to a-adrenergic agonists and an impaired relaxation to the $\beta$-adrenoceptor agonist isoproterenol, the endothelium-dependent vasodilator acetylcholine and the endotheliumindependent vasodilator SNP.

The DA belongs to a specialized system of $\mathrm{O}_{2}$-sensitive organs and tissues in the body that includes, among others, the pulmonary arteries, the carotid body, and the neuroepithelial body, which share striking similarities in the ways they respond to changes in $\mathrm{O}_{2}$ tension $(16,17)$. The mechanism proposed to explain $\mathrm{O}_{2}$-induced ductal contraction includes a sensor: the electron transport chain of 
the mitochondria that increases production of reactive oxygen species, particularly $\mathrm{H}_{2} \mathrm{O}_{2}$, in response to changes in $\mathrm{O}_{2}$ levels. $\mathrm{H}_{2} \mathrm{O}_{2}$ can reach the cell membrane and decrease the opening of $\mathrm{K}_{V}$ channels. This causes DA depolarization, opening of the voltage-gated $\mathrm{Ca}^{2+}$ channels, increase in intracellular $\mathrm{Ca}^{2+}$, and vasoconstriction $(16,17)$. Preliminary results of our laboratory indicate that this "mitochondria- $\mathrm{H}_{2} \mathrm{O}_{2}-\mathrm{K}_{v}$ channels" axis is also involved in the response of chicken DA to $\mathrm{O}_{2}$ (8, and unpublished results). Although chronic hypoxia could putatively alter each component of the above axis (18), we have not observed any hypoxia-induced alteration of the chicken DA response to oxygen. Moreover, the contractions induced by high- $\mathrm{K}^{+}$ depolarizing solution, the $\mathrm{K}_{v}$ channel blocker 4-AP, and $\mathrm{H}_{2} \mathrm{O}_{2}$ were not significantly affected by chronic hypoxia.

Within the vasculature in general, chronic hypoxia produces a broad spectrum of structural and functional changes that are typically organ specific (18). As reported elsewhere, chronic moderate hypoxia in the chicken fetus led, in the femoral artery, to impairment of endothelium-dependent relaxation $(6,7)$ and increased periarterial sympathetic innervation (5), whereas in the pulmonary arteries led to an impairment of the responsiveness to several vasoconstrictors but did not affect endothelium-dependent or -independent relaxations (7). In the present work, we observed that chronic hypoxia impaired ACh- and SNPmediated relaxations of the DA. Previously, we demonstrated that ACh induces endothelium-dependent relaxation of the chicken DA and that $\mathrm{NO}$ and endothelium derived hyperpolarizing factor are involved in this response (9). On the other hand, SNP evoked endothelium-independent relaxation of the chicken DA through the activation of soluble guanylate cyclase (sGC) (9). Therefore, the $\mathrm{NO} / \mathrm{sGC/cGMP}$ axis is active in the chicken DA and our present results indicate that chronic hypoxia induced an impairment of this vasodilatory pathway. It should be noted that, in the present work, we studied the effects of relaxant agonists in DA rings contracted with high- $\mathrm{K}^{+}$depolarizing solution. Thus, the contribution of hyperpolarizing mechanisms to relaxation could not be analyzed in our experiments. In relation to the vascular effects of chronic hypoxia, numerous investigations have examined the involvement of the NO/sGC/cGMP axis in pulmonary and systemic vessels. The majority of these studies report hypoxia-induced endothelial impairment with normal response to NO donors $(19,20)$. However, other authors report alterations in both endotheliumdependent and -independent relaxation after exposure to chronic hypoxia (21). In turn, chronic hypoxia has been reported to elevate vascular SGC abundance and activity in some studies (12), but decrease it in others (22). In the present study, we observed that the relaxation induced by the cell-permeable analog of 
cGMP, 8-Br-cGMP, was similar in the DA from normoxic and hypoxic fetuses, indicating that signaling events coupling cGMP to relaxation were not affected by chronic hypoxia. Taking together, our results suggest that chronic hypoxiainduced impairment of NO-evoked relaxation is due to a reduced SGC activity. However, an additional impairment of the endothelial NO synthase or an increase in phosphodiesterase-mediated catalysis of cGMP cannot be discarded.

Our study also reports that adrenergic agonist-induced contractions to norepinephrine and phenylephrine were enhanced in the DA from chicken fetuses exposed to chronic hypoxia during incubation. On the other hand, the DA relaxations induced by the $\beta$-adrenoceptor agonist isoproterenol were impaired in the hypoxic fetuses. Accordingly, other investigators reported that femoral artery constriction in response to adrenergic agonists was significantly enhanced in femoral arteries from rats (23) and lambs (14) gestated under hypoxia. In contrast, chronic hypoxia impaired the contraction evoked by aadrenergic agonists in pulmonary arteries (14). Previously, we demonstrated that norepinephrine and phenylephrine induced a developmentally increased contraction of the chicken DA (8). Interestingly, the relaxation induced by isoproterenol decreased with development (10). Therefore, the changes induced by hypoxia in DA adrenergic responsiveness mimic the changes induced by development, suggesting that the effect of hypoxia might be consequence of a developmental acceleration. In addition, the impairment of ACh- and SNP-mediated relaxation, which is a characteristic feature of the mature chicken DA (9), was also observed in the hypoxic fetuses. However, other features of the mature chicken DA, such as the enhanced responsiveness to $\mathrm{O}_{2}, \mathrm{KCl}, \mathrm{H}_{2} \mathrm{O}_{2}$, ET-1, or 4-AP $(8,24)$ were not present in the DA of the hypoxic animals.

Activation of the RhoA/Rho-kinase pathway can induce $\mathrm{Ca}^{2+}$ sensitization, a phenomenon in which sustained vasoconstriction occurs, independent of ongoing increases in cytosolic $\mathrm{Ca}^{2+}(25,26)$. Recently, Rho kinase activation has been implicated in DA constriction in several mammalian species $(25,26)$. Our observation of the Rho-kinase inhibitors Y-27632 and hydroxyfasudil concentration dependently decreasing tone of $\mathrm{KCl}$ - precontracted chicken $\mathrm{DA}$ suggests that this pathway also plays a role in the regulation of DA contraction in this species. Numerous experimental evidences indicate that chronic hypoxia augments Rho kinase-induced vascular smooth muscle $\mathrm{Ca}^{2+}$ sensitization, particularly in the pulmonary vasculature $(13,27)$ McNamara et al. (13) reported that pulmonary vascular RhoA is activated and Rho-kinase activity is increased in rat pups by exposure to hypoxia from birth for $14 \mathrm{~d}$. Moreover, hypoxia- 
induced RhoA expression in the lung is age dependent and found to be greatest in the fetus (27). In the present study, we observed that the relaxation evoked by Y-27632 and hydroxyfasudil was similar in DAs from normoxic and hypoxic fetuses. This indirectly suggests that Rho-kinase activity in the chicken DA was unaffected by chronic prenatal hypoxia.

The chicken DA presents morphologic and functional heterogeneity along its path between the pulmonary artery and the aorta $(8,24)$. Thus, the pulmonary side shows the structure of a muscular artery and responds to $\mathrm{O}_{2}$ with contraction, whereas the aortic part shows the morphology of an elastic artery and relaxes in response to $\mathrm{O}_{2}(8,24)$. In addition, ACh, SNP, and the NOindependent stimulator of SGC BAY 41-2272 induced larger relaxations in the aortic side of the vessel (9), whereas isoproterenol, forskolin, and the phosphodiesterase 3 inhibitor milrinone evoked larger relaxations in the pulmonary side (10). The reactivity studies of the present work were exclusively focused on the pulmonary side, which appears to be the "real" DA in the chicken. Bergwerff et al. (28) demonstrated that the distal elastic part of the chicken DA is mesodermal in origin and is the result of the incorporation of dorsal aorta tissue, whereas the muscular pulmonary side was shown to consist almost exclusively of neural crest derived cells. In the present work, we observed that the proportion of DA with muscular morphology was significantly reduced in the hypoxic animals. In addition, and although the in situ diameter of the DA was not affected by hypoxia, the functional diameter (i.e., the one at which the vessels showed maximal $\mathrm{K}^{+-i n d u c e d}$ contraction) was higher in the hypoxic group. With our present results, we can only speculate about these findings. However, and interestingly, numerous studies demonstrated that exposing animals to chronic hypoxia results in morphologic and functional changes in the neural crest-derived, oxygen-sensing cells of the carotid body (29). The specific effects of chronic hypoxia in the neural crest-derived cells of the DA and in the vascular elastic properties warrant further investigation.

As mentioned in the introduction, there have been reported signs of accelerated (3) or abnormal (4) DA development in preterm infants with IUGR. Recently, Rakza et al. (2) described that the DA is larger in infants with IUGR than in eutrophic preterm infants, as soon as $6 \mathrm{~h}$ after birth. In our chicken model, and with the exception of the above discussed shortening of the pulmonary segment, no other signs of morphologic alterations were found and the CSAs of the DA lumen and wall were comparable between normoxic and hypoxic 19-d fetuses. This suggests that at this stage of development the DA maintains a similar degree of patency in both groups. The 19-d fetuses used in the present study were noninternally pipped. On day 19 of incubation, the fetus internally 
pips by piercing the air cell inner membrane with its beak and begins lung ventilation. During the internal pipping, the relative blood flow to the chorioallantoic membrane progressively declines, whereas blood flow to the lungs increases (24). However, the presence of the ductal shunt is still necessary during internal pipping. In fact, Belanger et al. (24), reported that internal pipping did not induce significant changes in chicken DA diameter but these changes were observed during external pipping. At this stage, ductal closure was associated with a breakdown of the internal elastic lamina, migration of smooth muscle cells into the neointimal zone, and swelling and detachment of the endothelial layer $(9,24)$. By day 2 posthatching the lumen of the proximal portion of the DA is completely occluded (24). Whether the structural and functional alterations that we described in the present work accelerate or delay this process remains to be investigated. 


\section{References}

1. Fang S 2005 Management of preterm infants with intrauterine growth restriction. Early Hum Dev 81:889-900

2. Rakza T, Magnenant E, Klosowski S, Tourneux P, Bachiri A, Storme L 2007 Early hemodynamic consequences of patent ductus arteriosus in preterm infants with intrauterine growth restriction. J Pediatr 151:624-628

3. King DT, Emmanouilides GC, Andrews JC, Hirose FM 1980 Morphologic evidence of accelerated closure of the ductus arteriosus in preterm infants. Pediatrics $65: 872-880$

4. Ibara S, Tokunaga M, Ikenoue T, Murata Y, Hirano T, Asano H, Uemura Y 1994 Histologic observation of the ductus arteriosus in premature infants with intrauterine growth retardation. J Perinatol 14:411-416

5. Ruijtenbeek K, le Noble FA, Janssen GM, Kessels CG, Fazzi GE, Blanco CE, De Mey JG 2000 Chronic hypoxia stimulates periarterial sympathetic nerve develop- ment in chicken embryo. Circulation 102:2892-2897

6. Ruijtenbeek K, Kessels LC, De Mey JG, Blanco CE 2003 Chronic moderate hypoxia and protein malnutrition both induce growth retardation, but have distinct effects on arterial endothelium-dependent reactivity in the chicken embryo. Pediatr Res 53:573579

7. Villamor E, Kessels CG, Ruijtenbeek K, van Suylen RJ, Belik J, de Mey JG, Blanco CE 2004 Chronic in ovo hypoxia decreases pulmonary arterial contractile reactivity and induces biventricular cardiac enlargement in the chicken embryo. Am J Physiol Regul Integr Comp Physiol 287:R642-R651

8. Agren P, Cogolludo AL, Kessels CG, Perez-Vizcaino F, De Mey JG, Blanco CE, Villamor E 2007 Ontogeny of chicken ductus arteriosus response to oxygen and vasoconstrictors. Am J Physiol Regul Integr Comp Physiol 292:R485-R496

9. Agren P, van der Sterren S, Cogolludo AL, Frazziano G, de Mey JG, Blanco CE, Villamor E 2008 Developmental changes in endothelium-dependent relaxation of the chicken ductus arteriosus. J Physiol Pharmacol 59:55-76

10.Agren P, van der Sterren S, Cogolludo AL, Blanco CE, Villamor E 2008 Developmental changes in the effects of prostaglandin $E(2)$ in the chicken ductus arteriosus. $J$ Comp Physiol [B] [Epub ahead of print]

11. Marino M, Beny JL, Peyter AC, Bychkov R, Diaceri G, Tolsa JF 2007 Perinatal hypoxia triggers alterations in $\mathrm{K}+$ channels of adult pulmonary artery smooth muscle cells. Am J Physiol Lung Cell Mol Physiol 293:L1171-L1182

12.Li D, Laubach VE, Johns RA 2001 Upregulation of lung soluble guanylate cyclase during chronic hypoxia is prevented by deletion of eNOS. Am J Physiol Lung Cell Mol Physiol 281:L369 -L376

13.McNamara PJ, Murthy P, Kantores C, Teixeira L, Engelberts D, van Vliet T, Kavanagh BP, Jankov RP 2008 Acute vasodilator effects of Rho-kinase inhibitors in neonatal rats with pulmonary hypertension unresponsive to nitric oxide. Am J Physiol Lung Cell Mol Physiol 294:L205-L213 
14.Herrera EA, Pulgar VM, Riquelme RA, Sanhueza EM, Reyes RV, Ebensperger G, Parer JT, Valdez EA, Giussani DA, Blanco CE, Hanson MA, Llanos AJ 2007 Highaltitude chronic hypoxia during gestation and after birth modifies cardiovas- cular responses in newborn sheep. Am J Physiol Regul Integr Comp Physiol 292:R2234 R2240

15.Kajino H, Goldbarg S, Roman C, Liu BM, Mauray F, Chen YQ, Takahashi Y, Koch CJ, Clyman RI 2002 Vasa vasorum hypoperfusion is responsible for medial hypoxia and anatomic remodeling in the newborn lamb ductus arteriosus. Pediatr Res 51:228 235

16.Sutendra G, Michelakis ED 2007 The chicken embryo as a model for ductus arteriosus developmental biology: cracking into new territory. Am J Physiol Regul Integr Comp Physiol 292:R481-R484

17. Thebaud B, Wu XC, Kajimoto H, Bonnet S, Hashimoto K, Michelakis ED, Archer SL 2008 Developmental absence of the $\mathrm{O} 2$ sensitivity of L-type calcium channels in preterm ductus arteriosus smooth muscle cells impairs $\mathrm{O} 2$ constriction contributing to patent ductus arteriosus. Pediatr Res 63:176 -181

18.Paffett ML, Walker BR 2007 Vascular adaptations to hypoxia: molecular and cellular mechanisms regulating vascular tone. Essays Biochem 43:105-119

19.Peyter AC, Muehlethaler V, Liaudet L, Marino M, Di Bernardo S, Diaceri G, Tolsa JF 2008 Muscarinic receptor M1 and phosphodiesterase 1 are key determinants in pulmonary vascular dysfunction following perinatal hypoxia in mice. Am J Physiol Lung Cell Mol Physiol 295:L201-L213

20.Phillips SA, Olson EB, Morgan BJ, Lombard JH 2004 Chronic intermittent hypoxia impairs endothelium-dependent dilation in rat cerebral and skeletal muscle resistance arteries. Am J Physiol Heart Circ Physiol 286:H388 - H393

21.Xue Q, Ducsay CA, Longo LD, Zhang L 2008 Effect of long-term high-altitude hypoxia on fetal pulmonary vascular contractility. J Appl Physiol 104:1786-1792

22. Hassoun PM, Filippov G, Fogel M, Donaldson C, Kayyali US, Shimoda LA, Bloch KD 2004 Hypoxia decreases expression of soluble guanylate cyclase in cultured rat pulmonary artery smooth muscle cells. Am J Respir Cell Mol Biol 30:908 -913

23.Williams SJ, Campbell ME, McMillen IC, Davidge ST 2005 Differential effects of maternal hypoxia or nutrient restriction on carotid and femoral vascular function in neonatal rats. Am J Physiol Regul Integr Comp Physiol 288:R360 -R367

24.Belanger C, Copeland J, Muirhead D, Heinz D, Dzialowski EM 2008 Morphological changes in the chicken ductus arteriosi during closure at hatching. Anat Rec (Hoboken) 291:1007-1015

25.Kajimoto H, Hashimoto K, Bonnet SN, Haromy A, Harry G, Moudgil R, Nakanishi T, Rebeyka I, Thebaud B, Michelakis ED, Archer SL 2007 Oxygen activates the Rho/Rhokinase pathway and induces RhoB and ROCK-1 expression in human and rabbit ductus arteriosus by increasing mitochondria-derived reactive oxygen species: a newly recognized mechanism for sustaining ductal constriction. Circulation 115:1777-1788

26.Clyman RI, Waleh N, Kajino H, Roman C, Mauray F 2007 Calcium-dependent and 
calcium-sensitizing pathways in the mature and immature ductus arteriosus. Am $\mathrm{J}$ Physiol Regul Integr Comp Physiol 293:R1650 -R1656

27.Bailly K, Ridley AJ, Hall SM, Haworth SG 2004 RhoA activation by hypoxia in pulmonary arterial smooth muscle cells is age and site specific. Circ Res 94:13831391

28.Bergwerff M, Verberne ME, DeRuiter MC, Poelmann RE, Gittenberger-de Groot AC 1998 Neural crest cell contribution to the developing circulatory system: implications for vascular morphology? Circ Res 82:221-231

29.Wang ZY, Bisgard GE 2005 Postnatal growth of the carotid body. Respir Physiol Neurobiol 149:181-190 
Chapter 4

\title{
Prenatal exposure to hyperoxia modifies the thromboxane prostanoid receptor- mediated response to $\mathrm{H}_{2} \mathrm{O}_{2}$ in the ductus arteriosus of the chicken embryo
}

\author{
S. van der Sterren, L. Kessels, \\ F. Perez-Vizcaino, A.L. Cogolludo, E. Villamor \\ Journal of physiology and pharmacology 2014, 65, 2, 283-293
}




\begin{abstract}
$\mathrm{O}_{2}$ tension plays a critical role in the control of prenatal patency and postnatal closure of the ductus arteriosus (DA). We hypothesized that exposure of chicken embryos to hyperoxia alters the morphology and function of DA. Hyperoxia was induced by incubating fertilized eggs at $60 \% \mathrm{O}_{2}$ from day 15 to 19 of the 21-d incubation period. DA reactivity (assessed by wire myography), morphometry and mRNA expression of antioxidant enzymes were studied on day 19. Hyperoxic incubation neither affected embryonic growth nor induced signs of DA constriction or changed the mRNA expression of superoxide dismutase and catalase. The contractions induced by $\mathrm{O}_{2}(21 \%), \mathrm{KCl}, 4-$ aminopyridine, phenylephrine, and endothelin-1 and the relaxations induced by acetylcholine (ACh), sodium nitroprusside, isoproterenol, and hydroxyfasudil were similar in DA from embryos incubated under normoxic or hyperoxic conditions. In contrast, hyperoxic incubation impaired the thromboxane prostanoid (TP) receptor-mediated contractions evoked by U46619, 15- $\mathrm{E}_{2 t^{-}}$ Isoprostane and high concentrations $(\geq 3 \mu \mathrm{M})$ of $\mathrm{ACh}$. Exogenous hydrogen peroxide $\left(\mathrm{H}_{2} \mathrm{O}_{2}\right)$ evoked endothelium- dependent contraction in the normoxic DA and endothelium-dependent relaxation in the hyperoxic group. The presence of the TP receptor antagonist SQ 29548 unmasked a relaxant response to $\mathrm{H}_{2} \mathrm{O}_{2}$ in the normoxic DA and the cyclooxygenase (COX) inhibitor indomethacin blocked $\mathrm{H}_{2} \mathrm{O}_{2}$-induced contraction (in the normoxic group) and relaxation (in the hyperoxic group). Altogether our functional data suggest that, in the chicken DA, exogenous $\mathrm{H}_{2} \mathrm{O}_{2}$ induces the release of endothelium-derived COX metabolite(s) with contractile and relaxant properties. Under normal conditions $\mathrm{H}_{2} \mathrm{O}_{2}$-induced contraction prevails and relaxation is unmasked after pharmacological or functional (i.e. hyperoxia) TP receptor impairment.
\end{abstract}




\subsection{Introduction}

The ductus arteriosus (DA) is a large fetal vascular shunt connecting the pulmonary artery to the aorta, allowing most right ventricular blood to bypass the unexpanded lungs (1). $\mathrm{O}_{2}$ tension is a critical factor for the control of DA tone. In the fetus, the DA is tonically relaxed by the relatively hypoxic environment. In term infants, the increase in $\mathrm{O}_{2}$ tension at birth is a key factor stimulating DA constriction which precedes the anatomical and permanent closure of the vessel (2). In contrast, the DA from preterm infants is frequently unresponsive to $\mathrm{O}_{2}$ and less likely to constrict after birth. Failure of DA closure in very preterm infants is a common complication that can have significant clinical consequences and is still presenting challenges in terms of diagnosis, assessment and treatment options (2). Therefore, a better understanding of the biology and the pathobiology of the DA, and particularly of the DA responsiveness to $\mathrm{O}_{2}$, may have clinical implications.

In the last years the chicken embryo has emerged as a suitable model for the study of DA vascular biology $(1,3)$. Our group and another laboratory have characterized the developmental changes in the responsiveness of the chicken DA to $\mathrm{O}_{2}$ as well as other vasoactive agents including nitric oxide (NO), prostaglandins (PGs), and catecholamines (3-15). Some of these studies provide supportive evidence for a conserved mechanism for $\mathrm{O}_{2}$ sensing/ signaling in mammalian and non- mammalian DA $(1,3,6,15)$. Moreover, similarly to the mammalian DA $(2,16)$, the responsiveness of chicken DA to $\mathrm{O}_{2}$ is developmentally regulated, being very weak or absent at day 15 (E15) but present at day 19 (E19) of the 21 days of incubation $(3,8,13)$.

Critical time windows exist during development, and if environmental changes are experienced in the window of vulnerability, the developmental trajectory of the responding organ may be changed in a transient or a permanent way (17). DA smooth muscle cells belong to those specialized cell types that sense local $\mathrm{O}_{2}$ tension, which include glomus cells of the carotid body, neuroepithelial cells in the lungs and smooth muscle cells of the resistance pulmonary arteries and of the fetoplacental arteries $(18,19)$. A common feature of these specialized $\mathrm{O}_{2}$ sensing cells is that fetal and perinatal $\mathrm{O}_{2}$ levels may modulate their developmental programs. For example, either hypoxia or hyperoxia around the time of birth and the first few weeks thereafter can disturb the normal development of the mammalian carotid body leading to an altered ventilatory response to hypoxia that may persist until adulthood (20-23). Perinatal hypoxia/ hyperoxia can also disturb the normal development of the pulmonary vasculature and may predispose to altered pulmonary vasoconstrictor 
responses to hypoxia in later life (24-27). In the chicken embryo, exposure to hypoxia (28) or hyperoxia (29) during the last third of incubation blunted the hypoxic ventilatory chemosensitivity of the 1-day old hatchling.

In the present study we hypothesized that exposure to hyperoxia $\left(60 \% \mathrm{O}_{2}\right)$ alters the developmental trajectory of the chicken embryo DA resulting in structural and/or functional changes in the vessel. Therefore, we aimed to analyze the effects of hyperoxic incubation on DA morphometry, mRNA expression of antioxidant enzymes and ex vivo response to $\mathrm{O}_{2}$ and other vasoactive agents. In fact, the above hypothesis has been already investigated by Copeland and Dzialowski (15). However, in their study they exposed the embryos to $30 \% \mathrm{O}_{2}$ during the whole incubation period. In our experimental design, the embryos were exposed to a higher level of $\mathrm{O}_{2}$ and only during the critical period in which $\mathrm{O}_{2}$ responsiveness appears to undergo its development (i.e., between $\mathrm{E} 15$ and $\mathrm{E} 19)(3,6,13)$.

\subsection{Methods}

\subsubsection{Egg incubation and hyperoxia protocol}

All experimental procedures were carried out according to the regulations of the Dutch Law on Animal Experimentation and the European Directive for the Protection of Vertebrate Animals Used for Experimental and Other Scientific Purposes (86/609/EU).

Fertilized eggs of White Leghorn chickens (Gallus gallus) (Het Anker BV, Ochten, The Netherlands) were incubated at a temperature of $37.8^{\circ} \mathrm{C}, 45 \%$ air humidity and automatically rotated once every hour over an angle of $90^{\circ}$ (incubator model 25HS, Masalles Comercial, Spain). The $\mathrm{O}_{2}$ and $\mathrm{CO}_{2}$ concentrations in the incubators were monitored with a $\operatorname{DrDAQ} \mathrm{O}_{2}$ sensor (Pico Technology, United Kingdom) and an infrared $\mathrm{CO}_{2}$ analyzer (Beckman Instruments, Inc., Fullerton, CA, USA). Control eggs were incubated under normoxic conditions $\left(21 \% \mathrm{O}_{2}, 0.03 \% \mathrm{CO}_{2}\right)$. In the hyperoxia protocol, eggs were incubated under normoxia until day 15 and then transferred to a second incubator where hyperoxic conditions $\left(60 \pm 1 \% \mathrm{O}_{2}, 0.03 \% \mathrm{CO}_{2}\right)$ were maintained by providing a constant flow of $\mathrm{O}_{2}$, as previously described (30). Exposure of the egg to $60 \% \mathrm{O}_{2}$ leads to a significant change in arterial blood $\mathrm{Po}_{2}$ of chicken embryos (to $\sim 7 \mathrm{kPa}, \Delta \sim 3.8 \mathrm{kPa}$ ) similar to the transition of $\mathrm{Po}_{2}$ levels at birth (31). 


\subsubsection{Morphometric analysis of the ductus arteriosus}

The chicken DA is the result of the fusion of two vessels with different embryological origin, morphology and functionality. The pulmonary side consists almost exclusively of neural crest derived cells, shows the structure of a muscular artery and responds to $\mathrm{O}_{2}$ with contraction, whereas the aortic part is of mesodermal origin, shows the morphology of an elastic artery and relaxes in response to $\mathrm{O}_{2}(1,3,6,32)$. The studies of the present work were exclusively focused on the pulmonary side of the DA that is the 'real' DA in the chicken embryo, whereas the aortic side is the result of the incorporation of dorsal aorta tissue (32). Morphometric analysis was assessed in E19 DAs fixed in situ with the whole-body freezing technique. Embryos were frozen in $1 \%$ carboxymethylcellulose in liquid nitrogen. Serial, transversal sections (8 $\mu \mathrm{m}$ thick) through the whole length of the DAs, were obtained on a Leica freezing microtome (CM 3050S) and double staining was performed for elastin (Lawson solution; Klinipath, Duiven, The Netherlands) and a monoclonal antibody against smooth muscle a-actin (Dako, Carpinteria, CA, USA; diluted 1:300 and using diaminobenzidine as the chromogen). Microscopic images were analyzed with a computerized morphometric system (Quantimet 570C; Leica, Cambridge, UK) to determine media layer cross- sectional area (CSA), media wall thickness, and intraluminal CSA. This analysis was only performed in the right DA because its orientation allowed the observation of transversal sections of the vessel. From each specimen, 3-5 sections with the morphology of the pulmonary side of the DA were analyzed. The mean of the 3-5 measurements was used for computation. The morphometric analysis was performed by a single observer (L.K.), who was unaware of the experimental conditions.

\subsubsection{RT-PCR analysis}

Total RNA was isolated and purified from DA homogenates using RNeasy Fibrous Tissue Mini Kit (Qiagen). Total RNA was reverse transcribed into cDNA using iScript ${ }^{\mathrm{TM}}$ cDNA Synthesis Kit (BioRad) following manufacturer's instructions. Real-time PCR was performed using a Taqman system (RocheApplied Biosystems, Mannheim, Germany) in the Unidad de Genomica (Universidad Complutense de Madrid). Specific primers were designed for chicken superoxide dismutase 1 (SOD1, right 5'- GGTCCGGTAAGAGAAATGACAG-3' and left 5'-GACCTCGGCAATGTGACTG-3'), superoxide dismutase 2 (SOD2, right 5'-ATATGACCCCCACCATTGAA-3' and left 5'-GCTGGCAAAAGGTGATGTTAC-3'), catalase (right 5'-TGGATCCTTCAAATGAGTCTGA-3' and left 5'-GATGCAATGTTGTTTCCATCC-3') and GADPH (right 5'-GTCCTCTCTGGCAAAGTCCA-3' and left 5'-ACCATGTAGTTCAGATCGATGAAG-3') (6). The gene 
expression was calculated relative to the expression of the housekeeping gene GADPH, normalized by the average data under normoxic conditions for each gene using the comparative threshold cycle $\left(C_{\mathrm{T}}\right)$ method (33) and expressed as relative quantitation $(R Q)$ fold change.

\subsubsection{Recording of vascular reactivity}

E19 embryos were harvested, decapitated and fixed on a dissection plate. A midline laparotomy and sternotomy were performed and, with the aid of a dissecting microscope, the right and the left DAs were carefully dissected and divided pulmonary and aortic segments. Reactivity was analyzed only in the pulmonary side of the DA. Rings from either right or left DA were studied. Two stainless steel wires (diameter $40 \mu \mathrm{m}$ ) were inserted into the lumen of the vessels, which were mounted as ring segments between an isometric force transducer and a displacement device in a myograph (A/S model 610M; Danish Myo Technology, Aarhus, Denmark). Isometric tension signals were collected in real time (4 samples/s) and analyzed offline using a data acquisition system (PowerLab/4S and Chart 5 software; AD Instruments, Colorado Springs, CO, USA). The myograph organ bath (5-mL vol.) was filled with Krebs-Ringer bicarbonate (KRB) buffer, maintained at $39^{\circ} \mathrm{C}$. After a 30-minute equilibration period, the DA rings were distended to a resting tension corresponding to a transmural pressure of $20 \mathrm{mmHg}$. This pressure corresponds to the mean arterial blood pressure reported in chicken embryos at the corresponding age and elicits the highest contractile response to $\mathrm{KCl}$, as determined in previous experiments $(10-12,14)$. The vessels were allowed a 30 minutes equilibration period under basal tone, before eliciting a control contraction by raising the $\mathrm{K}^{+}$ concentration of the buffer to $62.5 \mathrm{mM}$ in exchange for $\mathrm{Na}+$. During this first phase of mounting and stabilization, rings were maintained in KRB buffer aerated with $95 \% \mathrm{~N}_{2} / 5 \% \mathrm{CO}_{2}\left(\mathrm{Po}_{2}=2.6-3.3 \mathrm{kPa}\right)(4,11,13)$.

The response of the DA rings to normoxia was assessed by bubbling the organ chamber with $21 \% \mathrm{O}_{2} / 74 \% \mathrm{~N}_{2} / 5 \% \mathrm{CO}_{2}\left(\mathrm{Po}_{2}=18.9-20.1 \mathrm{kPa}\right)$, as previously described $(3,11,13)$. The other contractile and relaxant agents were studied under $5 \% \mathrm{O}_{2} / 90 \% \mathrm{~N}_{2} / 5 \% \mathrm{CO}_{2}\left(\mathrm{Po}_{2}=6.8-7.2 \mathrm{kPa}\right)(4,11,13)$. The vasoactive stimuli were chosen based on their previously demonstrated activity in the chicken DA (3-15). Concentration-response curves to $\mathrm{KCl}(4.75 \mathrm{mM}-125 \mathrm{mM})$, the voltage-gated $\mathrm{K}^{+}$channel $\left(\mathrm{K}_{\mathrm{v}}\right)$ inhibitor 4-aminopyridine (4-AP, $1 \mathrm{mM}-10$ $\mathrm{mM})$, the $\mathrm{a}_{1}$-adrenoceptor agonist phenylephrine (10 nM-0.3 mM), endothelin-1 $(\mathrm{ET}-1,0.01 \mathrm{nM}-0.1 \mu \mathrm{M})$, the muscarinic receptor agonist acetylcholine (ACh, 3 $\mu \mathrm{M}-0.1 \mathrm{mM}$ ) the thromboxane prostanoid (TP) receptor agonist 9,11-dideoxy11a,9a-epoxymethano-prostaglandin $\mathrm{F}_{2 \mathrm{a}}(\mathrm{U} 46619,10 \mathrm{nM}-10 \mu \mathrm{M})$ and the 
isoprostane (IsoP) 15- $\mathrm{E}_{2 \mathrm{t}}$ IsoP $(10 \mathrm{nM}-10 \mu \mathrm{M})$ were constructed. Relaxant agents were evaluated during contraction induced by $125 \mathrm{mM} \mathrm{K}$. Concentration-response curves for ACh $(10 \mathrm{nM}-3 \mu \mathrm{M})$, the nitric oxide (NO) donor sodium nitroprusside (SNP, $10 \mathrm{nM}-0.1 \mathrm{mM}$ ), the $\beta$-adrenoceptor agonist isoproterenol $(0.1 \mathrm{nM}-3 \mu \mathrm{M})$, and the Rho-kinase inhibitor hydroxyfasudil (1 $\mathrm{nM}-30 \mu \mathrm{M}$ ) were constructed. Each DA ring was exposed to a maximum of three concentration- response curves. Between two curves the tissues were washed three times with fresh KRB buffer and allowed to equilibrate for $30 \mathrm{~min}$. The order of the concentration-response curves was similar in the normoxic and the hyperoxic group.

Some experiments (see results section) were performed in endotheliumdenuded vessels. The endothelium was removed by gentle rubbing of the vessel lumen with a horse tail hair and its effective removal was confirmed by the absence of relaxation when $1 \mu \mathrm{M}$ ACh was applied to DA rings contracted with $\mathrm{KCl} 62.5 \mathrm{mM}$. Other experiments were performed in the presence of the dual cyclooxygenase (COX) inhibitor indomethacin $(10 \mu \mathrm{M})$, the soluble guanylate cyclase inhibitor $1 \mathrm{H}[1,2,4]$ oxadiazolo[4,3-a]quinoxalin-1-one (ODQ, $10 \mu \mathrm{M})$ or the TP receptor antagonist SQ $29548(10 \mu \mathrm{M})$.

\subsubsection{Drugs and solutions}

KRB contained (in mmol L-1): $\mathrm{NaCl}$ (118.5), $\mathrm{KCl}$ (4.75), $\mathrm{MgSO}_{4}$ (1.2), $\mathrm{KH}_{2} \mathrm{PO}_{4}$ (1.2), $\mathrm{NaHCO}_{3}$ (25.0), $\mathrm{CaCl}_{2}$ (2.5) and glucose (5.5). Solutions containing different concentrations of $\mathrm{K}^{+}$were prepared by replacing $\mathrm{NaCl}$ by an equimolar amount of KCl. 15- $\mathrm{E}_{2 t}-\mathrm{IsoP}, \mathrm{U} 46619$ and SQ 29548 were obtained from Cayman Chemical (Ann Arbor, MI, USA). All other drugs were obtained from Sigma Chemical (St. Louis, MO, USA). Drugs were initially dissolved in distilled deionized water (except indomethacin that was dissolved in ethanol and $15-E_{2 t}-$ IsoP, U46619, SQ 29548 and ODQ that were dissolved in DMSO) to prepare stock solutions and further dilutions were made in $\mathrm{KRB} . \mathrm{H}_{2} \mathrm{O}_{2}$ solution was prepared by serial dilution of $30 \%$ stock solution with distilled deionized water. Total DMSO added to the organ bath for preincubations or concentrationresponse curves was, at the most, $0.1 \% \mathrm{v} / \mathrm{v}$ and did not affect arterial tone. Total ethanol was, at the most, $0.1 \% \mathrm{v} / \mathrm{v}$ and produced a slight contraction of the DA, as previously described (3).

\subsubsection{Data analysis}

Results are presented as mean \pm S.E.M. of measurements in $n$ embryos. Measurements of vascular reactivity were obtained from the mean of 40 samples (10 s) during the plateau phase of the response. Contractions are 
expressed in terms of active wall tension $(\mathrm{mN} / \mathrm{mm})$, calculated as the force divided by twice the length of the arterial segment. Relaxant responses are expressed as the percentage of reduction of the $\mathrm{KCl}$-induced contraction. A value $>100 \%$ means that the vessel was relaxed below the basal tone that existed before the addition of $\mathrm{KCl}$. Sensitivity/potency (expressed as $\mathrm{pEC}_{50}=-$ $\left.\log \mathrm{EC}_{50}\right)$ and efficacy $\left(E_{\max }\right)$ were calculated by nonlinear regression analysis of concentration- response curves. When the response to a vasoactive compound did not conform to sigmoidal curve fitting, responses at individual concentrations were compared. Differences between mean values were assessed by unpaired $t$-tests or one-way ANOVA, followed by Bonferroni post hoc test. Differences were considered significant at $P<0.05$. All analyses were performed using a commercially available statistics package (Graphpad Prism version 5, GraphPad InStat version 3.00; GraphPad Software Inc, San Diego, CA, USA).

\subsection{Results}

\subsubsection{Embryonic growth and ductus arteriosus morphometry}

Exposure of chicken embryos to $60 \%$ instead of $21 \% \mathrm{O}_{2}$ from day 15 to day 19 of incubation did not affect embryonic growth (body mass normoxic: 29.6 \pm 0.34 $\mathrm{g}, \mathrm{n}=41$; hyperoxic : $29.9 \pm 0.37 \mathrm{~g}, n=42, P=0.55$ versus normoxic).

As shown in Figs. $4.1 A$ and $4.1 B$, the media and lumen CSAs of the right DA were not significantly different between normoxic and hyperoxic embryos. Therefore, hyperoxic incubation did not induce evident signs of DA constriction.

\subsubsection{Ductus arteriosus reactivity}

Switching the $\mathrm{O}_{2}$ mixture from 0 to $21 \%$ produced a progressive increase of the $\mathrm{O}_{2}$ concentration in the organ chamber that reached a steady state after $\sim 3 \mathrm{~min}$ (3). This increase in $\mathrm{O}_{2}$ concentration resulted in a contractile response of the DA that was not significantly different between normoxic $(0.29 \pm 0.04 \mathrm{mN} / \mathrm{mm})$ and hyperoxic $(0.22 \pm 0.03 \mathrm{mN} / \mathrm{mm})$ embryos (Fig. $4.2 A)$. Also the contractions induced by 4-AP (Fig. 4.2B; maximal contraction normoxia: $0.56 \pm 0.05 \mathrm{mN} / \mathrm{mm}$; maximal contraction hyperoxia: $0.72 \pm 0.12 \mathrm{mN} / \mathrm{mm}$ ), $\mathrm{KCl}$ (Fig. $4.2 C ; E_{\max }$ normoxia: $0.52 \pm 0.05 \mathrm{mN} / \mathrm{mm}$; $E_{\max }$ hyperoxia: $0.72 \pm 0.10 \mathrm{mN} / \mathrm{mm}$ ), phenylephrine (Fig. 4.2D; $E_{\max }$ normoxia: $0.68 \pm 0.07 \mathrm{mN} / \mathrm{mm}$; $E_{\max }$ hyperoxia: $0.73 \pm 0.10 \mathrm{mN} / \mathrm{mm}$ ) and ET-1 (Fig. $2 E, E_{\max }$ normoxia: $0.42 \pm 0.10 \mathrm{mN} / \mathrm{mm}$; $E_{\max }$ hyperoxia: $0.49 \pm 0.07 \mathrm{mN} / \mathrm{mm}$ ) did not vary between the two groups. In the chicken DA, the response to the muscarinic agonist ACh is biphasic, causing 
A

Normoxia
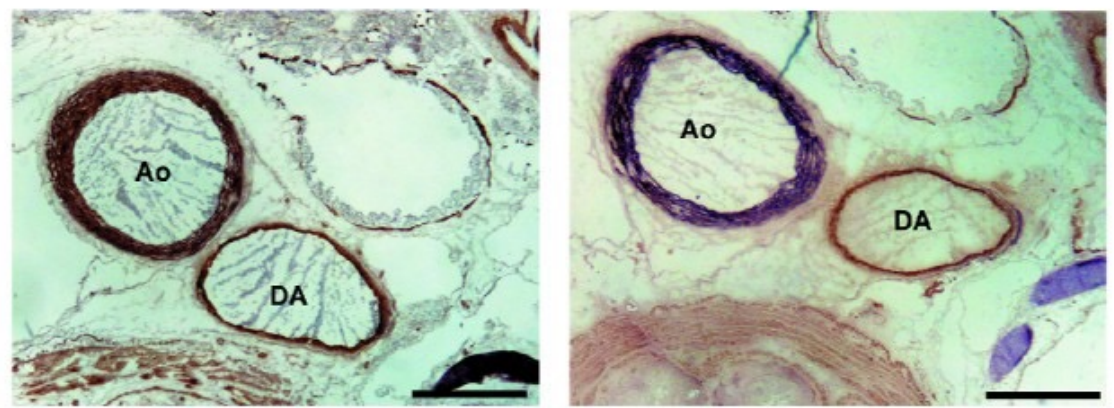

B

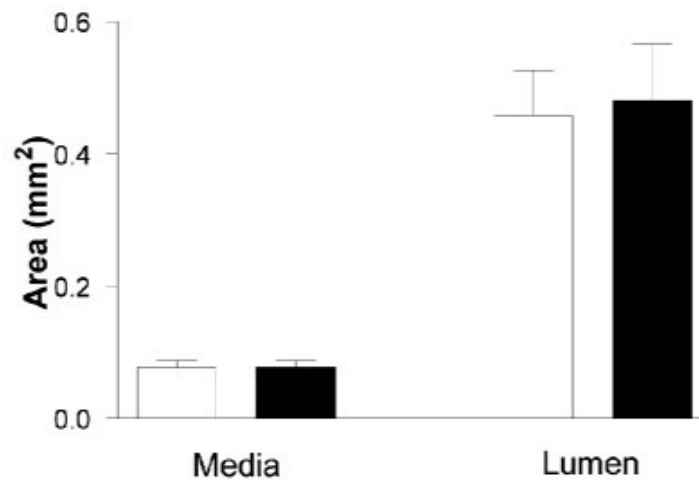

Fig. 4.1. $(A)$ : Light micrographs showing double staining for elastin and a-actin of cross sections of snap- frozen 19-day chicken embryos incubated under normoxia $\left(21 \% \mathrm{O}_{2}\right)$ or hyperoxia $\left(60 \% \mathrm{O}_{2}\right)$ from day 15 until day 19 of the 21-day chicken incubation period. Ao: aorta; DA: ductus arteriosus. Bars $500 \mu \mathrm{M}$. (B): The media and lumen cross sectional areas of the right DA were not significantly different between normoxic and hyperoxic 19-day embryos. From each specimen, 3-5 sections with the morphology of the pulmonary side of the ductus arteriosus were analyzed and the mean value of the 3-5 sections was taken as the value for each embryo. Each bar represents the mean \pm S.E.M. of 5 embryos.

relaxation (see next paragraph) and contraction respectively at low and high concentrations (12). The contraction evoked by high concentrations ( $>3 \mu \mathrm{M}$ ) of ACh contraction was significantly impaired in the hyperoxic embryos (Fig. 4.2F; $E_{\max }$ normoxia: $0.33 \pm 0.06 \mathrm{mN} / \mathrm{mm}$; $E_{\max }$ hyperoxia: $0.12 \pm 0.02 \mathrm{mN} / \mathrm{mm}, P<0.01$ versus normoxia). In addition, the contractile potencies ( $\mathrm{EEC}_{50}$ ) of U46619 (Fig. 4.3A) and $15-\mathrm{E}_{2 \mathrm{t}}$ - IsoP (Fig. 4.3B) were significantly reduced in the DA from the hyperoxia group ( $\mathrm{pEC}_{50} \mathrm{U} 46619$ normoxia: $6.42 \pm 0.08 \mathrm{n}=8$; hyperoxia: $5.74 \pm 0.08, n=8, P<0.001$ versus normoxia), $\left(p E_{50} 15-E_{2 t}-I s o P\right.$ normoxia: 
$7.03 \pm 0.15, n=6$; hyperoxia: $5.87 \pm 0.12, n=6, P<0.01$ versus normoxia). The contractile efficacies of U46619 ( $E_{\max }$ normoxia: $1.14 \pm 0.12 \mathrm{mN} / \mathrm{mm}$; $E_{\max }$ hyperoxia: $0.92 \pm 0.16 \mathrm{mN} / \mathrm{mm}$ ) and $15-E_{2 t}-$ lsoP $\left(E_{\max }\right.$ normoxia: $1.08 \pm 0.10 \mathrm{mN} /$ $\mathrm{mm}$; $E_{\max }$ hyperoxia: $1.02 \pm 0.16 \mathrm{mN} / \mathrm{mm}$ ) were not significantly different between the two groups (Fig. $3 A-3 B$ ).

A

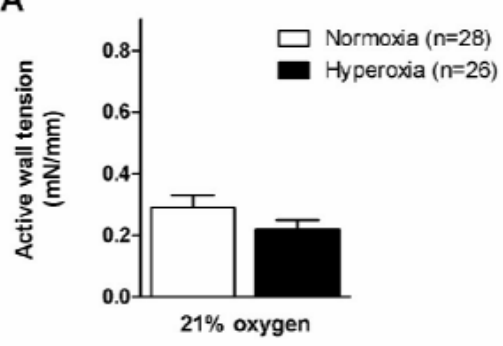

C

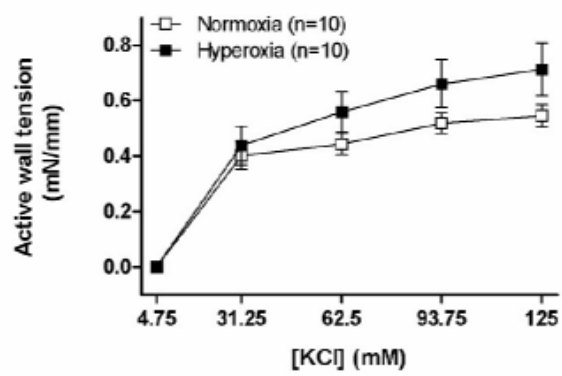

E

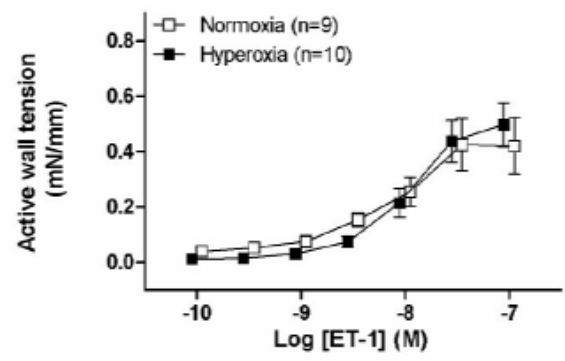

B

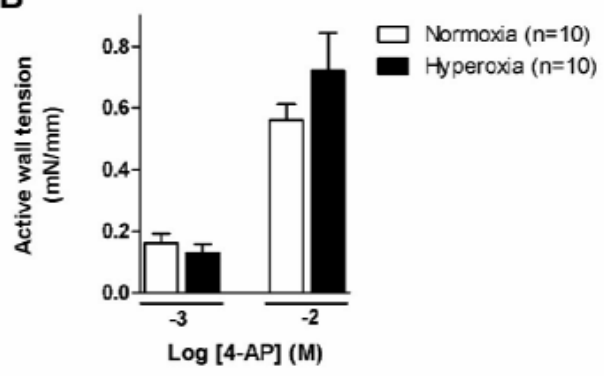

D

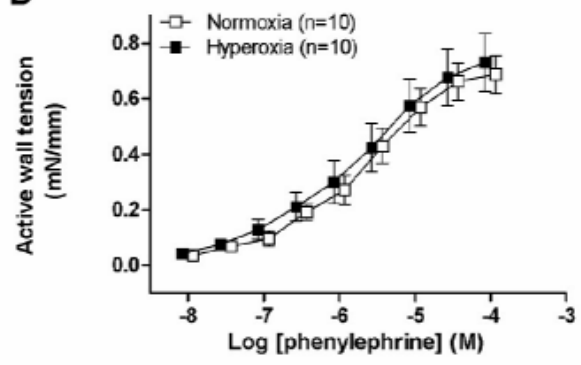

$\mathbf{F}$

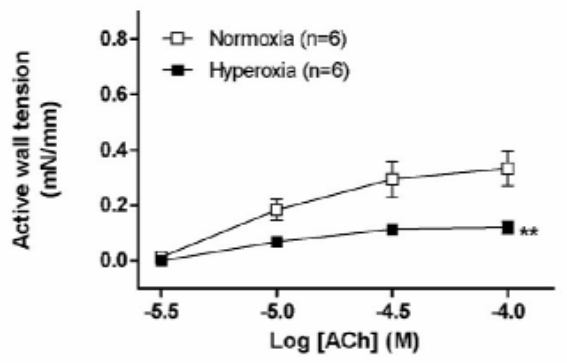

Fig. 4.2. Contractile effects of $\mathrm{O}_{2}(A)$, 4-aminopyridine (4-AP, $\left.B\right), \mathrm{KCl}(C)$, phenylephrine $(D)$, endothelin-1 (ET- 1, E) and acetylcholine (ACh, $F$ ) in ductus arteriosus rings (pulmonary side) of 19day chicken embryos incubated under normoxia $\left(21 \% \mathrm{O}_{2}\right)$ or hyperoxia $\left(60 \% \mathrm{O}_{2}\right)$ from day 15 until day 19 of the 21-day chicken incubation period. Ductus arteriosus rings were mounted in a myograph under isometric conditions. Each point (or bar) represents the mean \pm S.E.M. of $n$ embryos. Overlapped symbols were slightly shifted horizontally to make them visible.

${ }^{* *} P<0.01$ for difference in $E_{\max }$ from normoxia. 
A

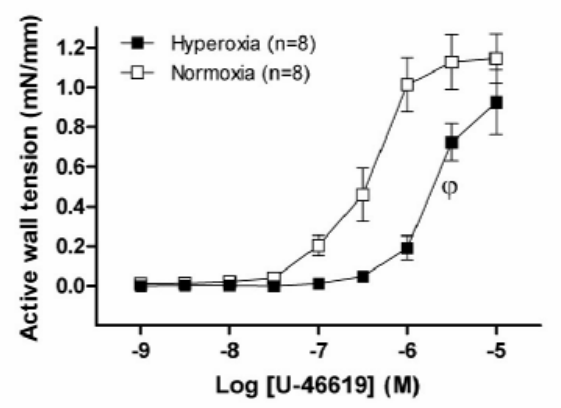

B

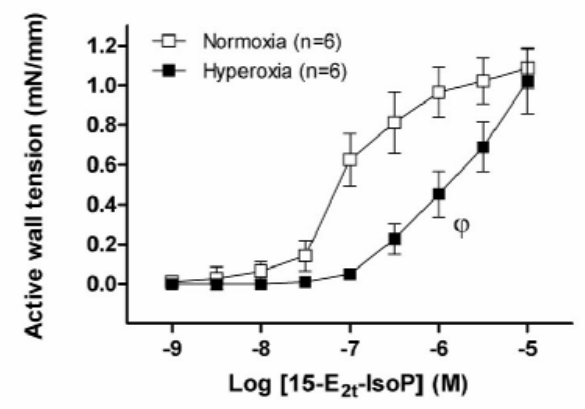

Fig. 4.3. Concentration-dependent contractile effects of the TP receptor agonists U46619 $(A)$ and 15-E2t-Isoprostane $(B)$ in ductus arteriosus rings (pulmonary side) of 19-day chicken incubated under normoxia $\left(21 \% \mathrm{O}_{2}\right)$ or hyperoxia $\left(60 \% \mathrm{O}_{2}\right)$ from day 15 until day 19 of the 21-day chicken incubation period. Ductus arteriosus rings were mounted in a myograph under isometric conditions. Each point represents the mean \pm S.E.M. of $n$ embryos. Overlapped symbols were slightly shifted horizontally to make them visible. $\varphi P<0.01$ for difference in $\mathrm{pEC}_{50}$ from normoxia. Significance symbol is placed approximately adjacent to the $\mathrm{pEC}_{50}$ value.
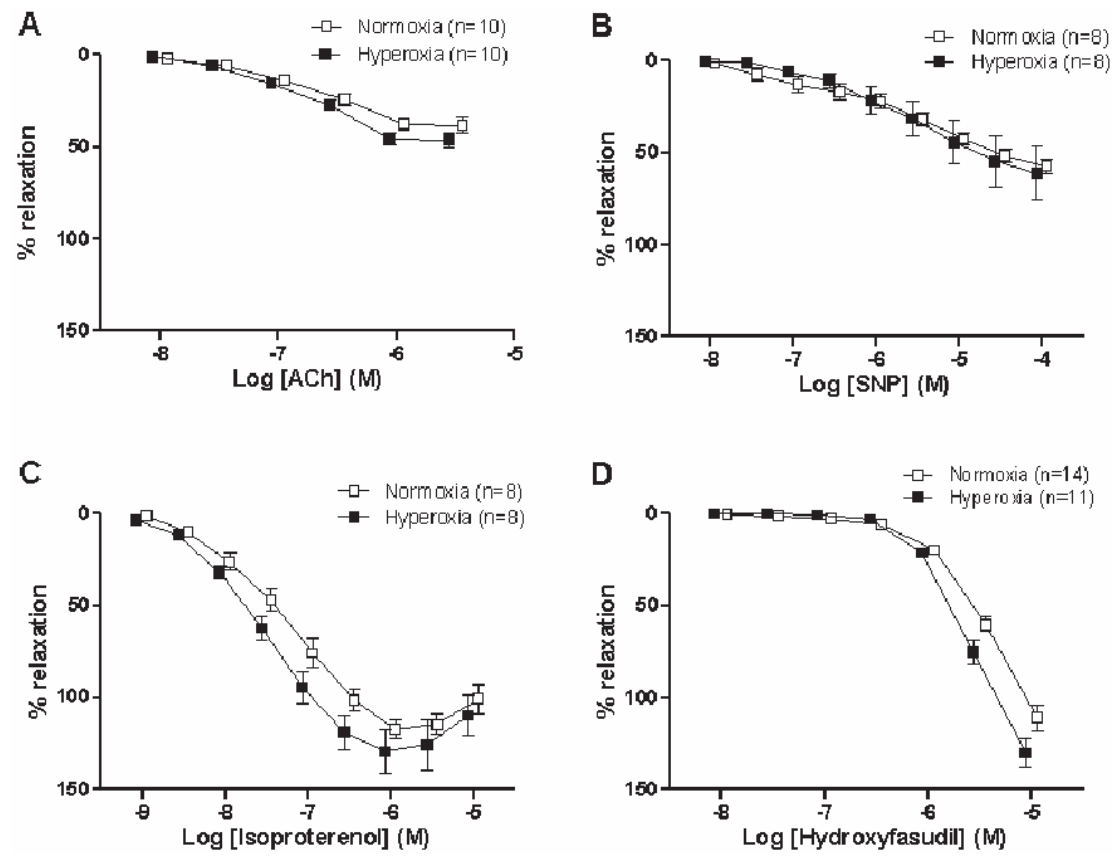

Fig. 4.4. Concentration-dependent relaxant effects of acetylcholine (ACh, $A$ ), sodium nitroprusside (SNP, $B$ ), isoproterenol $(C)$, and hydroxyfasudil $(D)$ in ductus arteriosus rings (pulmonary side) of 19day chicken embryos incubated under normoxia $\left(21 \% \mathrm{O}_{2}\right)$ or hyperoxia $\left(60 \% \mathrm{O}_{2}\right)$ from day 15 until day 19 of the 21-day chicken incubation period. Ductus arteriosus rings were mounted in a myograph under isometric conditions and contracted with $\mathrm{KCl}(125 \mathrm{mM})$ before the cumulative concentration-response curves were obtained. Each point represents the mean \pm S.E.M. of $n$ embryos. Overlapped symbols were slightly shifted horizontally to make them visible. 
ACh (10 nM-3 $\mu \mathrm{M}$, Fig. 4A), SNP (Fig. 4.4B), isoproterenol (Fig. 4.4C), and hydroxyfasudil (Fig. 4.4D) relaxed DA rings (precontracted with $125 \mathrm{mM} \mathrm{KCl}$ ) in a concentration-dependent manner. These relaxations were not significantly different when normoxic and hyperoxic DAs were compared. High concentrations of isoproterenol did not induce further relaxation but contraction (Fig. $4.4 C$ ) as previously described (4). Isoproterenol ( $E_{\max }$ normoxia: 117.30 $\pm 5.28 \%$; $E_{\max }$ hyperoxia: $129.48 \pm 11.90 \%$ ) and hydroxyfasudil ( $E_{\max }$ normoxia: $110.98 \pm 5.28 \%$; $E_{\max }$ hyperoxia: $130.21 \pm 7.66 \%$ ) relaxed the DA rings below the basal tone that existed before the addition of $\mathrm{KCl}$. This presumably reflects relaxation of myogenic tone. In contrast, ACh ( $E_{\max }$ normoxia: $38.21 \pm 4.04 \%$; $E_{\max }$ hyperoxia: $46.39 \pm 3.73 \%$ ) and $\operatorname{SNP}\left(E_{\max }\right.$ normoxia: $57.37 \pm 4.03 \%$; $E_{\max }$ hyperoxia: $61.3 \pm 14.61 \%$ ) showed a markedly lower relaxant efficacy.

Exogenous $\mathrm{H}_{2} \mathrm{O}_{2}$ contracted $D A$ rings from the normoxic group in a concentration-dependent manner (Figs. $4.5 \mathrm{~A}$ and $4.5 \mathrm{C}$ ). In contrast, $\mathrm{H}_{2} \mathrm{O}_{2}$ evoked a concentration-dependent relaxation in the DA from the hyperoxiaincubated embryos (Figs. $4.5 B$ and $4.5 C$ ). In order to understand these intriguing findings, we performed additional experiments to analyze 1) the putative changes induced by hyperoxia in the gene expression of antioxidant enzymes and 2) the mechanisms involved in the ductal effects of exogenous $\mathrm{H}_{2} \mathrm{O}_{2}$. As shown in Fig. 4.5D, the DA mRNA expression of SOD1, SOD2 and catalase were not significantly different between the normoxic and the hyperoxic group.

As shown in Fig. 4.6, either mechanical removal of the endothelium (Figs. 4.6A and $4.6 D$ ) or the presence of the dual COX-1 and COX-2 inhibitor indomethacin blunted $\mathrm{H}_{2} \mathrm{O}_{2}$-induced contraction in the normoxic group (Figs. 4.6B and 4.6D). Moreover, in the presence of the TP receptor antagonist SQ 29548, $\mathrm{H}_{2} \mathrm{O}_{2}$ evoked a concentration-dependent relaxation in the DA of normoxic chicken embryos (Figs. $4.6 \mathrm{C}$ and $4.6 \mathrm{D}$ ). The relaxation evoked by $\mathrm{H}_{2} \mathrm{O}_{2}$ in the hyperoxic DA was blunted after endothelium removal or incubation with indomethacin (Fig. 4.7). In contrast the presence of SQ 29548 did not affect the relaxant effect of $\mathrm{H}_{2} \mathrm{O}_{2}$ in the hyperoxic DA (Fig. 4.7). Finally, the presence of the soluble guanylate cyclase inhibitor ODQ did not induce significant changes in the ductal response to $\mathrm{H}_{2} \mathrm{O}_{2}$ of normoxic and hyperoxic embryos (data not shown). 
A

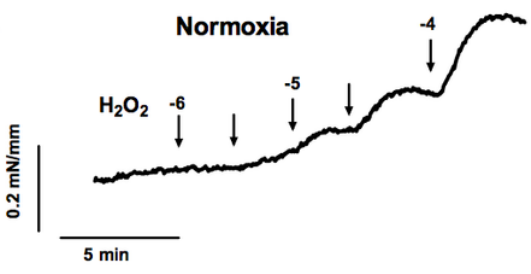

C

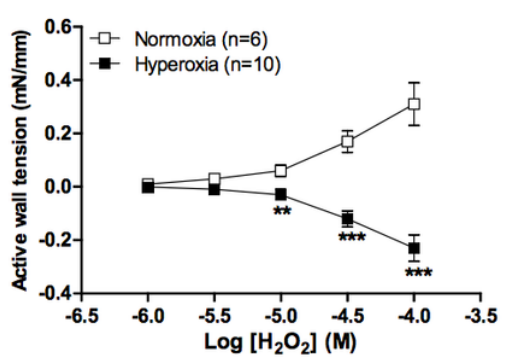

B
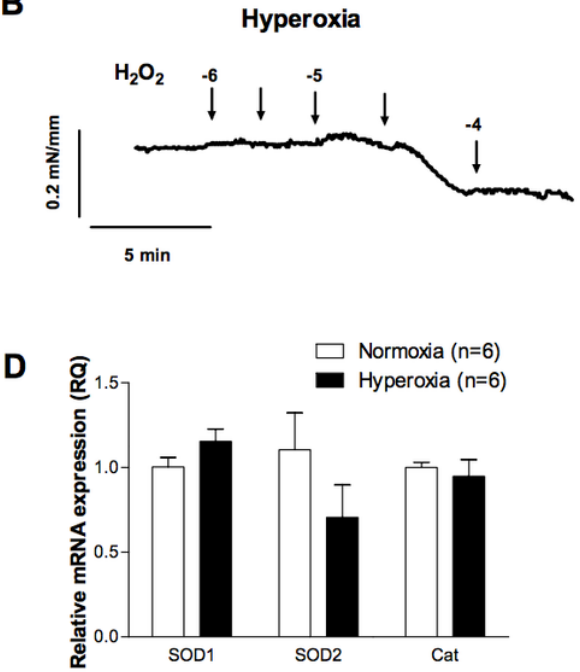

Fig. 4.5. Exogenous $\mathrm{H}_{2} \mathrm{O}_{2}$ evoked contraction in the $\mathrm{DA}$ of chicken embryos incubated under normoxia but relaxation in the DA from embryos incubated under hyperoxia. (A-B): Representative tracing of active wall tension versus time showing the response to $\mathrm{H} 2 \mathrm{O} 2$ of DA rings (pulmonary side) from 19-day chicken embryos in ductus arteriosus rings (pulmonary side) of 19-day chicken incubated under $(\mathrm{A})$ : normoxia $(21 \% \mathrm{O})$ or $(\mathrm{B})$ hyperoxia $(60 \% \mathrm{O})$ from day 15 until day 19 of the 21-day chicken incubation period. Ductus arteriosus rings were mounted in a myograph under isometric conditions. Values indicate log $\mathrm{M}$ [H2O2]. Unlabeled arrows represent half-log concentrations. (C): Mean ( \pm S.E.M.) cumulative dose-response curves for $\mathrm{H} 2 \mathrm{O} 2$ in DA rings from normoxic-incubated and hyperoxic-incubated chicken embryos. ${ }^{* *},{ }^{* * *} \mathrm{P}<0.01,0.001$ versus normoxia. Responses at individual concentrations were compared because the response to $\mathrm{H} 2 \mathrm{O} 2$ did not conform to sigmoidal curve fitting. (D): The mRNA expression of the antioxidant enzymes superoxide dismutase (SOD)1, SOD2 and catalase was not significantly affected by hyperoxic incubation. The gene expression was calculated relative to the expression of the housekeeping gene GADPH, normalized by the average data under normoxic conditions for each gene using the comparative $\mathrm{CT}$ method and expressed as relative quantitation $(\mathrm{RQ})$ fold change. 
A

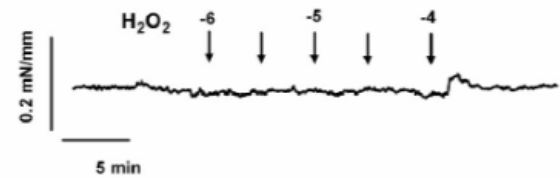

B

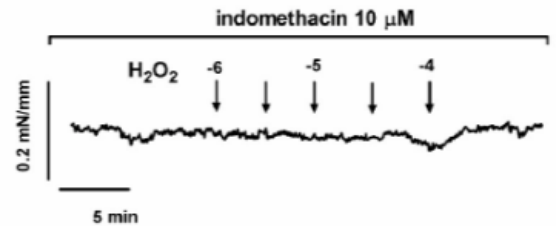

C

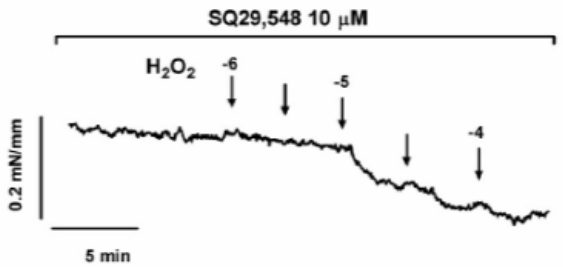

D

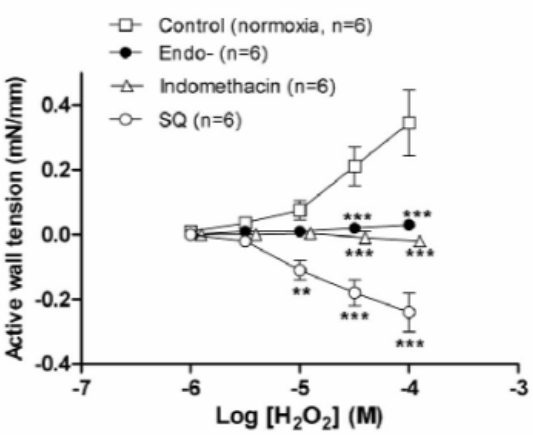

Fig. 4.6. $(A-C)$ : Representative tracing of active wall tension vs. time showing the response to exogenous $\mathrm{H}_{2} \mathrm{O}_{2}$ of DA rings (pulmonary side) from 19-day normoxic-incubated chicken embryos after endothelial denudation $(A)$ or in the presence of the isoform-nonspecific cyclooxygenase inhibitor indomethacin $(10 \mu \mathrm{M}, B)$ or the TP receptor antagonist SQ $29548(10 \mu \mathrm{M}, C)$. Ductus arteriosus rings were mounted in a myograph under isometric conditions. Values indicate log $M$ $\left[\mathrm{H}_{2} \mathrm{O}_{2}\right]$. Unlabeled arrows represent half-log concentrations. $(D)$ : Mean ( \pm S.E.M.) cumulative doseresponse curves for $\mathrm{H}_{2} \mathrm{O}_{2}$ in DA rings from 19-day normoxic-incubated chicken embryos after endothelial denudation or in the presence of SQ $29548(\mathrm{SQ})$, indomethacin or the soluble guanylate cyclase inhibitor ODQ $(10 \mu \mathrm{M})$. Overlapped symbols were slightly shifted horizontally to make them visible. ${ }^{* *},{ }^{* *} P<0.01,0.001$ versus control. Responses at individual concentrations were compared because the response to $\mathrm{H}_{2} \mathrm{O}_{2}$ did not conform to sigmoidal curve fitting. 


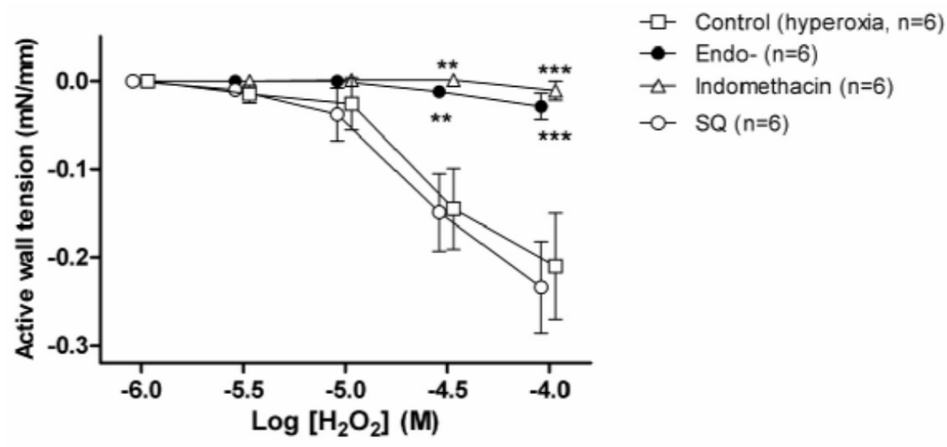

Fig. 4.7. Mean ( \pm S.E.M.) cumulative dose-response curves for $\mathrm{H}_{2} \mathrm{O}_{2}$ in ductus arteriosus rings (pulmonary side) of 19-day chicken incubated under hyperoxia $\left(60 \% \mathrm{O}_{2}\right)$ from day 15 until day 19 of the 21-day chicken incubation period. Ductus arteriosus rings were mounted in a myograph under isometric conditions. The effects of endothelial denudation (Endo-), the presence of the TP receptor antagonist SQ $29548(10 \mu \mathrm{M}, \mathrm{SQ})$ or the isoform-nonspecific cyclooxygenase inhibitor indomethacin $(10 \mu \mathrm{M})$ are also shown. Overlapped symbols were slightly shifted horizontally to make them visible. ${ }^{* *},{ }^{\star \star *} \mathrm{P}<0.01,0.001$ versus hyperoxia-incubated control. Responses at individual concentrations were compared because the response to $\mathrm{H}_{2} \mathrm{O}_{2}$ did not conform to sigmoidal curve fitting.

\subsection{Discussion}

We investigated the effects of hyperoxic incubation $\left(60 \% \mathrm{O}_{2}\right.$ between $\mathrm{E} 15$ and E19) of chicken embryos on the biology of the DA. We observed that hyperoxic incubation neither affected embryonic growth nor induced signs of DA constriction or changed the mRNA expression of antioxidant enzymes. The DA contractions evoked by $\mathrm{O}_{2}, \mathrm{KCl}, 4-\mathrm{AP}$, and $\mathrm{ET}-1$ and the relaxations induced by $\mathrm{ACh}, \mathrm{SNP}$, isoproterenol and hydroxyfasudil were similar in the normoxic and hyperoxic group. In contrast, hyperoxic incubation impaired the TP receptormediated contraction evoked by U46619, 15- $\mathrm{E}_{2 \mathrm{t}} \mathrm{IsOP}$ and ACh. Exogenous $\mathrm{H}_{2} \mathrm{O}_{2}$ evoked endothelium-dependent contraction in the normoxic DA and endothelium-dependent relaxation in the hyperoxic group. The presence of a TP receptor antagonist unmasked a relaxant response to $\mathrm{H}_{2} \mathrm{O}_{2}$ in the normoxic DA and the $\mathrm{COX}$ inhibitor indomethacin blocked $\mathrm{H}_{2} \mathrm{O}_{2}$-induced contraction (in the normoxic group) and relaxation (in the hyperoxic group). Altogether our functional data suggest that exogenous $\mathrm{H}_{2} \mathrm{O}_{2}$ induces the release of endothelium-based COX metabolites with contractile and relaxant properties in the chicken DA. Under normal conditions $\mathrm{H}_{2} \mathrm{O}_{2}$-induced contraction prevails but relaxation is unmasked after pharmacological or functional (i.e. hyperoxia) TP receptor impairment. 
4.4.1 Hyperoxic incubation of chicken embryos did not affect ductus arteriosus $\mathrm{O}_{2}$ responsiveness

Although incubation of eggs under $60 \% \mathrm{O}_{2}$ increases the blood $\mathrm{PO}_{2}$ of chicken embryos to post-hatch levels (31), we did not observe signs of DA constriction or changes in the DA responsiveness to $\mathrm{O}_{2}$ in E19 embryos. Transition to exovo life in chickens is initiated on around incubation day 20 when the embryo internally pips the air cell of the egg with its beak and begins breathing relatively hypoxic air cell gas $\left(\mathrm{Po}_{2} \sim 14.5 \mathrm{kPa}\right)(1,15)$. However, it is not until the embryo breaks the eggshell and begins to breath normoxic air during external pipping that the blood $\mathrm{Po}_{2}$ increases and the DA is stimulated to close $(1,9,15)$. We observed that $\mathrm{O}_{2}$ sensitivity was not altered by in ovo exposure to $60 \% \mathrm{O}_{2}$. Copeland and Dzialowski analyzed the responsiveness to $\mathrm{O}_{2}, \mathrm{KCl}$ and catecholamines (epinephrine and norepinephrine) in the DA of E16, E18, internally pipped E20 and externally pipped chicken embryos exposed to $30 \%$ $\mathrm{O}_{2}$ from the first day of incubation (15). In agreement with our results, they found that in the non-internally pipped $\mathrm{E} 18$ embryo $\mathrm{O}_{2^{-}}, \mathrm{KCl}-$, and catecholamine-induced contractions were not affected by hyperoxic incubation (15). In contrast, the DA from the hyperoxia-incubated E20 internally pipped embryos showed a higher $\mathrm{O}_{2}$-induced contraction than the normoxia-incubated age- matched controls. In addition, the DA from externally-pipped hyperoxic embryos was completely closed, whereas the DA from normoxic embryos was still open (15). In the study of Copeland and Dzialowski, hyperoxia was maintained during internal and external pipping and therefore the chicks were relying on pulmonary gas-exchange. It is only under these conditions that the response to $\mathrm{O}_{2}$ was increased and ductal closure accelerated (15). Taken together, these and our results indicate that high $\mathrm{O}_{2}$ tension in chicken embryos relying on choriallantoic respiration does not stimulate constriction of the relatively immature E19 DA. In addition, the developmental trajectory of the $\mathrm{O}_{2}$ sensitive machinery of the DA is not affected by early and prolonged exposure to $\mathrm{O}_{2}$. In contrast, using a very different experimental setting, Michelakis et al. found that 72 hours of exposure to $\mathrm{O}_{2}$ induced depolarization and reduced expression of $\mathrm{O}_{2}$-sensitive $\mathrm{K}^{+}$channels in human $\mathrm{DA}$ smooth muscle cells, resulting in selective failure to constrict in response to $\mathrm{O}_{2}(34)$.

4.4.2 Hyperoxic incubation reduced ductus arteriosus responsiveness to thromboxane prostanoid receptor stimulation

Although a chicken ortholog for the human TP receptor gene has not yet been found (35), chicken platelets (36), leukocytes (37) and blood vessels (12) produce thromboxane. Moreover, the TP receptor agonist U46619 is an 
efficacious contractile agent in chicken embryo pulmonary (11, 38), mesenteric $(39,40)$, femoral $(11)$ and chorioallantoic $(19,41)$ arteries as well as in the DA $(3,11,12)$. Other prostanoids such as $P_{G} E_{2}$ or the IsoPs $15-E_{2 t}$-IsoP and 15$F_{2 t}$ IsoP also evoke TP receptor-mediated contraction in the chicken DA $(4,11)$. Finally, high concentrations of ACh induced in the chicken DA an endotheliumdependent contraction, which involved COX-1 activation and TP receptor stimulation (12). Therefore, TP receptor(s) are functionally present in the chicken DA and may play a pivotal role in the regulation of its vascular tone.

We found that the TP receptor-mediated contraction evoked by U46619, 15- $\mathrm{E}_{2 t^{-}}$ IsoP, and ACh was impaired in the DA from chicken embryos incubated under hyperoxia. Furthermore, $\mathrm{H}_{2} \mathrm{O}_{2}$ evoked a TP receptor-mediated contraction in the DA from normoxic embryos but relaxation in the hyperoxia group. This last point is discussed in more detail below. The relation between oxidative stress and vascular responsiveness to TP receptor stimulation is complex and with our present functional results we can only speculate on the mechanisms responsible for the hyporesponsiveness that we observed in the hyperoxic group. In addition, the chicken TP receptor has not been cloned, making not possible to analyze the changes in gene expression evoked by hyperoxia. Chronic alveolar hyperoxia impaired the pulmonary vasoconstriction elicited by TP receptor agonism in the adult sheep but the mechanisms responsible have not been clearly established (42). On the other hand, several studies demonstrate that $\mathrm{H}_{2} \mathrm{O}_{2}$-induced oxidative stress increases vascular TP sensitivity and predisposes blood vessels to constrictor prostanoids $(43,44)$. Interestingly, Belik et al. found that hyperoxia-induced pulmonary artery hyporesponsiveness to TP receptor stimulation was only present in the adult rat, whereas neonatal rats chronically exposed to $\mathrm{O} 2$ showed enhanced vascular and airway smooth muscle contraction to U46619 (45). However, it should be noted that the neonatal pulmonary artery hyperresponsiveness to U46619 was accompanied by increased response to $\mathrm{KCl}$ and reduced vascular endotheliumindependent relaxation (45), whereas we found that hyperoxia in the chicken DA selectively affected TP receptor- mediated contraction.

4.4.3 Normoxic ductus arteriosus contracted and hyperoxic ductus arteriosus relaxed in response to exogenous $\mathrm{H}_{2} \mathrm{O}_{2}$

$\mathrm{H}_{2} \mathrm{O}_{2}$, a membrane permeable and relatively stable reactive $\mathrm{O}_{2}$ species (ROS), has emerged as an important signaling molecule in the regulation of physiological and pathophysiological processes in vascular cells $(46,47)$. Extensive evidence points to a role for $\mathrm{H}_{2} \mathrm{O}_{2}$ in the normoxic contraction of mammalian and chicken DA. In the DA, $\mathrm{O}_{2}$ - induced constriction is thought to be 
mediated, at least in part, by the inhibition of $\mathrm{K}_{\mathrm{v}}$ channels, resulting in membrane depolarization, and $\mathrm{Ca}^{2+}$ entry through voltage operated $\mathrm{Ca}^{2+}$ channels $(48,49)$. Michelakis et al. $(34,49,50)$ proposed a model in which a rise in $\mathrm{O}_{2}$ modulates the function of the mitochondrial electron transport chain (ETC, the sensor), leading to an increased production of $\mathrm{H}_{2} \mathrm{O}_{2}$ (the mediator) which causes the inhibition of $\mathrm{K}_{v}$ channels (the effector). $\mathrm{H}_{2} \mathrm{O}_{2}$-mediated activation and induction of Rho kinase has been identified as another downstream effector of the $\mathrm{O}_{2}$-sensing system in mammalian DA (51-53). In the chicken DA, a similar mechanism implicating $\mathrm{H}_{2} \mathrm{O}_{2}$ as mediator of $\mathrm{O}_{2}$-induced contraction has been characterized $(6,8)$. Thus, $\mathrm{O}_{2}$ induced an increase in $\mathrm{H}_{2} \mathrm{O}_{2}$ production in the pulmonary part of chicken DA but not in the aortic side of the vessel (6). Moreover, $\mathrm{O}_{2}$-induced contraction of chicken DA was impaired by $\mathrm{H}_{2} \mathrm{O}_{2}$ scavengers $(6,8)$ and, in a striking similarity with $\mathrm{O}_{2}$, exogenous $\mathrm{H}_{2} \mathrm{O}_{2}$ contracted the pulmonary part of chicken DA and relaxed the aortic side of the vessel (6). Finally, the responses to $\mathrm{O}_{2}$ and exogenous $\mathrm{H}_{2} \mathrm{O}_{2}$ undergo a parallel maturation process between E15 and E19-20 (6).

Extracellular application of $\mathrm{H}_{2} \mathrm{O}_{2}$ appears to be directly relevant to biology and may mimic signaling by endogenously produced $\mathrm{H}_{2} \mathrm{O}_{2}(47,54,55)$. We observed that exogenous $\mathrm{H}_{2} \mathrm{O}_{2}$ evoked a contraction in the pulmonary side of the DA from normoxic embryos, as previously described $(6,7)$. In contrast, $\mathrm{H}_{2} \mathrm{O}_{2}$ relaxed the DA from the hyperoxia-incubated embryos. In order to understand this intriguing finding, we investigated the effects of hyperoxia on the mRNA expression of antioxidant enzymes and we further analyzed the mechanisms involved in the chicken DA response to exogenous $\mathrm{H}_{2} \mathrm{O}_{2}$. We discuss the former here and the latter in the next paragraph. Van Golde et al. demonstrated that incubation of chicken embryos under $60 \% \mathrm{O}_{2}$ produce an increase in the activity of SOD, catalase and glutathione peroxidase, which was dependent on the enzyme, organ (brain, heart, liver, intestine and lung), incubation time and time point of exposure (31). Intracellular $\mathrm{H}_{2} \mathrm{O}_{2}$ levels are lowered by catalase, by the reverse activity of SOD, by a range of peroxidases, and by passive and active diffusion into the extracellular space (54). Thus, hyperoxia-induced alterations in the antioxidant system of the DA might have led to accelerated or delayed $\mathrm{H}_{2} \mathrm{O}_{2}$ kinetics resulting in an alteration of the response to exogenous $\mathrm{H}_{2} \mathrm{O}_{2}$. However, this explanation is unlikely because we did not observe, at least at the level of mRNA expression, a significant induction of catalase or SOD in the chicken DA following hyperoxic incubation.

A large body of experimental evidence has accumulated demonstrating that exogenous $\mathrm{H}_{2} \mathrm{O}_{2}$ can cause either vasoconstriction or relaxation depending on species, vascular bed, contractile state of the vessel and presence of certain 
pathophysiological conditions $(6,46,56-60) . \mathrm{H}_{2} \mathrm{O}_{2}$-induced vasoconstriction has been related to stimulation of TP receptors by products of arachidonic acid metabolism via $\operatorname{COX}(47,57,59,61)$. It has also been linked to increased L-type $\mathrm{Ca}^{2+}$ channel activity (62) and to activation of protein kinases $(61,63)$. Conversely, the relaxation involves endothelium-dependent and -independent mechanisms, which could be mediated by nitric oxide (64), COX-derived relaxant prostaglandins $(47,65)$, or carbon monoxide (44). The signal transduction mechanisms involved in $\mathrm{H}_{2} \mathrm{O}_{2}$-induced relaxation include activation of guanylate cyclase with subsequent increase in cGMP and PKG activation (66), cGMP-independent mechanisms of PKG activation (66), increase in cAMP (60), or membrane hyperpolarization (67). We observed that the contraction in the normoxic DA and the relaxation in the hyperoxic DA were blunted by endothelium removal or COX inhibition. Interestingly, the presence of a TP receptor antagonist unmasked a relaxant response to $\mathrm{H}_{2} \mathrm{O}_{2}$, which was very similar to the one observed in the hyperoxic DA. The relaxation evoked by $\mathrm{H}_{2} \mathrm{O}_{2}$ was not affected by inhibition of guanylate cyclase. Altogether these data suggest that $\mathrm{H}_{2} \mathrm{O}_{2}$ induces the release of endothelium-derived $\mathrm{COX}$ metabolite(s) with contractile and relaxant properties in the chicken DA. The predominant action of $\mathrm{H}_{2} \mathrm{O}_{2}$ would be the TP receptor-mediated contraction but when this effect is impaired, such as is the case of hyperoxia or TP receptor antagonism, the relaxation is unmasked.

The identification of the COX product(s) responsible for the effects of $\mathrm{H}_{2} \mathrm{O}_{2}$ in the chicken DA warrants further investigation. As mentioned above, the vascular actions of $\mathrm{H}_{2} \mathrm{O}_{2}$ have been linked to the production of contractile and relaxant prostanoids. In the chicken embryo DA, $\mathrm{PGE}_{2}$ has been shown to induce TP receptor-mediated contraction and EP receptor-mediated relaxation (4). This makes this prostanoid a good candidate for explaining the divergent actions of exogenous $\mathrm{H}_{2} \mathrm{O}_{2}$ in the chicken DA. Work in progress in our laboratory is currently testing this hypothesis.

Another intriguing finding of our study is that despite the altered response to exogenous $\mathrm{H}_{2} \mathrm{O}_{2}$ observed in the hyperoxic DA, the contraction induced by $\mathrm{O}_{2}$ was not affected. These observations challenge the role of $\mathrm{H}_{2} \mathrm{O}_{2}$ as intermediate in $\mathrm{O}_{2}$-induced contraction of the chicken DA. However, it should be noted that although extracellular application of $\mathrm{H}_{2} \mathrm{O}_{2}$ appears to mimic the endogenous release of $\mathrm{H}_{2} \mathrm{O}_{2}$, endogenous $\mathrm{H}_{2} \mathrm{O}_{2}$ is frequently present at much lower concentrations and for less time than extracellular $\mathrm{H}_{2} \mathrm{O}_{2}(54,55)$. Moreover, during $\mathrm{O}_{2}$-induced contraction, $\mathrm{H}_{2} \mathrm{O}_{2}$ is produced in the DA smooth muscle cells where it directly exerts its action as mediator $(18,34)$. Accordingly, $\mathrm{O}_{2}$-induced contraction in the chicken DA is impaired by the presence of the $\mathrm{H}_{2} \mathrm{O}_{2}$ 
scavenger catalase but is not significantly affected by endothelium removal (6). In contrast, we observed that the contractile effects of exogenous $\mathrm{H}_{2} \mathrm{O}_{2}$ are endothelium-dependent. Therefore, despite producing the same effect as $\mathrm{O}_{2}$ in the chicken DA (i.e., contraction), exogenous $\mathrm{H}_{2} \mathrm{O}_{2}$ and $\mathrm{O}_{2}$ do not share a common transduction mechanism. As reviewed by Forman, exogenous $\mathrm{H}_{2} \mathrm{O}_{2}$ may mimic signaling by endogenously produced $\mathrm{H}_{2} \mathrm{O}_{2}$ and has the same advantage as using any other membrane-permeable second messenger (55). The primary disadvantage of its use is that the results can be misleading because $\mathrm{H}_{2} \mathrm{O}_{2}$ may produce other effects instead of or in addition to what the endogenously produced $\mathrm{H}_{2} \mathrm{O}_{2}$ can do (55). In order to assess the real role of $\mathrm{H}_{2} \mathrm{O}_{2}$, a combination of experimental approaches needs to be used and particular attention paid to the kinetics and concentration dependence of the reactions in which $\mathrm{H}_{2} \mathrm{O}_{2}$ is proposed to participate.

\subsubsection{Perspectives and significance}

Mammalian fetuses and avian embryos develop in a relatively hypoxic environment and it is under these conditions that the DA, a specialized $\mathrm{O}_{2-}$ sensitive tissue, undergoes its specific developmental program in preparation for postnatal closure. Infants born very prematurely are exposed to normoxia or even to hyperoxia when this developmental program is far from being completed. The new situation includes not only a higher $\mathrm{O}_{2}$ tension but also changes in the hemodynamic forces to which the vessel is subjected (i.e., pressure, velocity and direction of flow) as consequence of the circulatory adaptation to the replacement of the placenta by the lung as gas exchange organ (25). Under these new conditions the DA frequently fails to complete its developmental program, resulting in failure of postnatal closure. Hyperoxic incubation of chicken embryos allows isolating the effects of $\mathrm{O}_{2}$ in DA development without the interferences of maternal factors and in the presence of a prenatal circulatory pattern. Our study provides evidence that changes in $\mathrm{O}_{2}$ tension alters the normal development of the DA. However, the alterations induced by hyperoxia did not result in changes in $\mathrm{O}_{2}$ responsiveness of the vessel. Further investigations are warranted to analyze the contractile pathways that are down- or up-regulated by hyperoxia in the chicken DA and to explore whether changes in $\mathrm{O}_{2}$ tension affected the normal development of other specialized $\mathrm{O}_{2}$-sensitive vessels like the pulmonary (68) or the chorioallantoic arteries (19). 


\section{References}

1. Dzialowski EM, Sirsat T, van der Sterren S, Villamor E. Prenatal cardiovascular shunts in amniotic vertebrates. Respir Physiol Neurobiol 2011; 178: 66-74.

2. Clyman Rl. Mechanisms regulating the ductus arteriosus. Biol Neonate 2006; 89: 330-335.

3. Agren P, Cogolludo AL, Kessels CG, et al. Ontogeny of chicken ductus arteriosus response to oxygen and vasoconstrictors. Am J Physiol Regul Integr Comp Physiol 2007; 292: R485-R496.

4. Agren P, van der Sterren S, Cogolludo AL, Blanco CE, Villamor E. Developmental changes in the effects of prostaglandin E2 in the chicken ductus arteriosus. J Comp Biol B 2009; 179: 133-143.

5. Agren $\mathrm{P}$, van der Sterren $\mathrm{S}$, Cogolludo $\mathrm{AL}$, et al. Developmental changes in endothelium-dependent relaxation of the chicken ductus arteriosus. J Physiol Pharmacol 2008; 59: 55-76.

6. Cogolludo AL, Moral-Sanz J, van der Sterren S, et al. Maturation of O2 sensing and signaling in the chicken ductus arteriosus. Am J Physiol Lung Cell Mol Physiol 2009; 297: L619-L630.

7. Van der Sterren S, Agren P, Zoer B, Kessels L, Blanco CE, Villamor E. Morphological and functional alterations of the ductus arteriosus in a chicken model of hypoxia-induced fetal growth retardation. Pediatr Res 2009; 65: 279-284.

8. Greyner H, Dzialowski EM. Mechanisms mediating the oxygen-induced vasoreactivity of the ductus arteriosus in the chicken embryo. Am J Physiol Regul Integr Comp Physiol 2008; 295: R1647-R1659.

9. Belanger C, Copeland J, Muirhead D, Heinz D, Dzialowski EM. Morphological changes in the chicken ductus arteriosi during closure at hatching. Anat Rec (Hoboken) 2008; 291: 1007-1015.

10. Van der Sterren S, Kleikers P, Zimmermann LJ, Villamor E. Vasoactivity of the gasotransmitters hydrogen sulfide and carbon monoxide in the chicken ductus arteriosus. Am J Physiol Regul Integr Comp Physiol 2011; 301: R1186- R1198.

11. Van der Sterren S, Villamor E. Contractile effects of 15-E2t- isoprostane and 15-F2tisoprostane on chicken embryo ductus arteriosus. Comp Biochem Physiol A Mol Integr Physiol 2011; 159: 436-444.

12. Schuurman MJ, Villamor E. Endothelium-dependent contraction induced by acetylcholine in the chicken ductus arteriosus involves cyclooxygenase-1 activation and TP receptor stimulation. Comp Biochem Physiol A Mol Integr Physiol 2010; 157: 28-34.

13. Flinsenberg TW, van der Sterren $S$, van Cleef AN, Schuurman MJ, Agren P, Villamor

E. Effects of sex and estrogen on chicken ductus arteriosus reactivity. Am J Physiol Regul Integr Comp Physiol 2010; 298: R1217- 1224.

14. Moonen RM, Agren P, Cogolludo AL, Perez Vizcaino F, Villamor E. Response of chicken ductus arteriosus to hypercarbic and normocarbic acidosis. Neonatology 2010; 98: 47-56. 
15. Copeland J, Dzialowski EM. Effects of hypoxic and hyperoxic incubation on the reactivity of the chicken embryo (Gallus gallus) ductus arteriosi in response to catecholamines and oxygen. Exp Physiol 2009; 94: 152-161.

16. Thebaud B, Michelakis ED, Wu XC, et al. Oxygen-sensitive Kv channel gene transfer confers oxygen responsiveness to preterm rabbit and remodeled human ductus arteriosus: implications for infants with patent ductus arteriosus. Circulation 2004; 110: 1372-1379.

17. Nathanielsz PW. Animal models that elucidate basic principles of the developmental origins of adult diseases. Ilar J 2006; 47: 73-82.

18. Weir EK, Lopez-Barneo J, Buckler KJ, Archer SL. Acute oxygen-sensing mechanisms. N Engl J Med 2005; 353: 2042-2055.

19. Lindgren I, Zoer B, Altimiras J, Villamor E. Reactivity of chicken chorioallantoic arteries, avian homologue of human fetoplacental arteries. J Physiol Pharmacol 2010; 61: 619-628.

20. Erickson JT, Mayer C, Jawa A, et al. Chemoafferent degeneration and carotid body hypoplasia following chronic hyperoxia in newborn rats. J Physiol 1998; 509: 519-526.

21. Blanco CE, Chen V, Maertzdorf W, Bamford OS, Hanson M. Effect of hyperoxia (PaO2 50-90 $\mathrm{mmHg}$ ) on fetal breathing movements in the unanaesthetized fetal sheep. $J$ Dev Physiol 1990; 14: 235-241.

22. Ling L, Olson EB, Jr., Vidruk EH, Mitchell GS. Attenuation of the hypoxic ventilatory response in adult rats following one month of perinatal hyperoxia. J Physiol 1996; 495: 561-571.

23. Teppema LJ, Dahan A. The ventilatory response to hypoxia in mammals: mechanisms, measurement, and analysis. Physiol Rev 2010; 90: 675-754.

24. Sartori C, Allemann Y, Trueb L, Delabays A, Nicod P, Scherrer U. Augmented vasoreactivity in adult life associated with perinatal vascular insult. Lancet 1999; 353: 2205-2207.

25. Gao Y, Raj JU. Regulation of the pulmonary circulation in the fetus and newborn. Physiol Rev 2010; 90: 1291-1335.

26. Tang JR, Le Cras TD, Morris KG, Jr., Abman SH. Brief perinatal hypoxia increases severity of pulmonary hypertension after reexposure to hypoxia in infant rats. Am J Physiol Lung Cell Mol Physiol 2000; 278: L356-L364.

27. Rey-Parra GJ, Archer SL, Bland RD, et al. Blunted hypoxic pulmonary vasoconstriction in experimental neonatal chronic lung disease. Am J Respir Crit Care Med 2008; 178: 399-406.

28. Szdzuy K, Mortola JP. Ventilatory chemosensitivity of the 1- day-old chicken hatchling after embryonic hypoxia. Am J Physiol Regul Integr Comp Physiol 2007; 293: R1640R1649.

29. Mortola JP. Prenatal hyperoxia blunts the hypoxic ventilatory chemosensitivity of the 1-day old chicken hatchling. Respir Physiol Neurobiol 2011; 178: 352-356. 
30. Been JV, Zoer B, Kloosterboer N, et al. Pulmonary vascular endothelial growth factor expression and disaturated phospholipid content in a chicken model of hypoxia-induced fetal growth restriction. Neonatology 2010; 97: 183-189.

31. Van Golde JC, Borm PJ, Wolfs MC, Rhijnsburger EH, Blanco CE. Induction of antioxidant enzyme activity by hyperoxia $(60 \%$ O2) in the developing chick embryo.J Physiol 1998; 509: 289-296.

32. Bergwerff M, DeRuiter MC, Gittenberger-de Groot AC. Comparative anatomy and ontogeny of the ductus arteriosus, a vascular outsider. Anat Embryol 1999; 200: 559-571. 33. Schmittgen TD, Livak KJ. Analyzing real-time PCR data by the comparative $C(T)$ method. Nat Protoc 2008; 3: 1101-1108.

34. Suzuki K, Naoki K, Kudo H, et al. Impaired hypoxic vasoconstriction in intraacinar microvasculature in hyperoxia-exposed rat lungs. Am J Respir Crit Care Med 1998; 158 : 602-609.

34. Michelakis ED, Rebeyka I, Wu X, et al. O2 sensing in the human ductus arteriosus: regulation of voltage-gated $\mathrm{K}+$ channels in smooth muscle cells by a mitochondrial redox sensor. Circ Res 2002; 91: 478-486.

35. Lagerstrom MC, Hellstrom AR, Gloriam DE, Larsson TP, Schioth HB, Fredriksson R. The G protein-coupled receptor subset of the chicken genome. PLoS Comput Biol 2006; 2: e54.

36. Claeys M, Wechsung E, Herman AG, Nugteren DH. Lack of prostacyclin biosynthesis by aortic tissue of the chicken. Prostaglandins 1981; 21: 739-749.

37. Cherian G, Bautista-Ortega J, Goeger DE. Maternal dietary n-3 fatty acids alter cardiac ventricle fatty acid composition, prostaglandin and thromboxane production in growing chicks. Prostaglandins Leukot Essent Fatty Acids 2009; 80: 297-303.

38. Villamor E, Ruijtenbeek K, Pulgar V, De Mey JG, Blanco CE. Vascular reactivity in intrapulmonary arteries of chicken embryos during transition to ex ovo life. Am J Physiol Regul Integr Comp Physiol 2002; 282: R917-R927.

39. Moonen RM, Villamor E. Developmental changes in mesenteric artery reactivity in embryonic and newly hatched chicks. J Comp Physiol B 2011; 181: 1063-1073.

40. Moonen RM, Kessels CG, Zimmermann LJ, Villamor E. Mesenteric artery reactivity and small intestine morphology in a chicken model of hypoxia-induced fetal growth restriction. J Physiol Pharmacol 2012; 63: 601-612.

41. Lindgren I, Crossley D, Villamor E, Altimiras J. Hypotension in the chronically hypoxic chicken embryo is related to the beta-adrenergic response of chorioallantoic and femoral arteries and not to bradycardia. Am J Physiol Regul Integr Comp Physiol 2011; 301: R1161-R1168.

42. Newman JH, Loyd JE, English DK, Ogletree ML, Fulkerson WJ, Brigham KL. Effects of $100 \%$ oxygen on lung vascular function in awake sheep. J Appl Physiol 1983; 54: 1379- 1386.

43. Wilson SJ, Cavanagh CC, Lesher AM, Frey AJ, Russell SE, Smyth EM. Activationdependent stabilization of the human thromboxane receptor: role of reactive oxygen species. J Lipid Res 2009; 50: 1047-1056. 
44. Nitin P, Zhang F, Monu SR, et al. Antioxidants condition pleiotropic vascular responses to exogenous $\mathrm{H} 2 \mathrm{O} 2$ : role of modulation of vascular TP receptors and the heme oxygenase system. Antiox Redox Signal 2012; 18: 471-480.

45. Belik J, Jankov RP, Pan J, Tanswell AK. Chronic O2 exposure enhances vascular and airway smooth muscle contraction in the newborn but not adult rat. J Appl Physiol 2003; 94: 2303-2312.

46. Perez-Vizcaino F, Cogolludo A, Moreno L. Reactive oxygen species signaling in pulmonary vascular smooth muscle. Respir Physiol Neurobiol 2010; 174: 212-220.

47. Korbecki J, Baranowska-Bosiacka I, Gutowska I, Chlubek D. The effect of reactive oxygen species on the synthesis of prostanoids from arachidonic acid. J Physiol Pharmacol 2013; 64: 409-421.

48. Tristani-Firouzi M, Reeve HL, Tolarova S, Weir EK, Archer SL. Oxygen-induced constriction of rabbit ductus arteriosus occurs via inhibition of a 4-aminopyridine-, voltage-sensitive potassium channel. J Clin Invest 1996; 98: 1959-1965.

49. Michelakis E, Rebeyka I, Bateson J, Olley P, Puttagunta L, Archer S. Voltage-gated potassium channels in human ductus arteriosus. Lancet 2000; 356: 134-137.

50. Sutendra G, Michelakis ED. The chicken embryo as a model for ductus arteriosus developmental biology: cracking into new territory. Am J Physiol Regul Integr Comp Physiol 2007; 292: R481-R484.

51. Clyman Rl, Waleh N, Kajino H, Roman C, Mauray F. Calcium-dependent and calcium-sensitizing pathways in the mature and immature ductus arteriosus. Am $\mathrm{J}$ Physiol Regul Integr Comp Physiol 2007; 293: R1650-R1656.

52. Hong Z, Hong F, Olschewski A, et al. Role of store-operated calcium channels and calcium sensitization in normoxic contraction of the ductus arteriosus. Circulation 2006; 114: 1372-1379.

53. Kajimoto $\mathrm{H}$, Hashimoto $\mathrm{K}$, Bonnet $\mathrm{SN}$, et al. Oxygen activates the Rho/Rho-kinase pathway and induces RhoB and ROCK-1 expression in human and rabbit ductus arteriosus by increasing mitochondria-derived reactive oxygen species: a newly recognized mechanism for sustaining ductal constriction. Circulation 2007; 115: 17771788.

54. Schroder E, Eaton P. Hydrogen peroxide as an endogenous mediator and exogenous tool in cardiovascular research: issues and considerations. Curr Opin Pharmacol 2008; 8: 153-159.

55. Forman HJ. Use and abuse of exogenous $\mathrm{H} 2 \mathrm{O} 2$ in studies of signal transduction. Free Radic Biol Med 2007; 42: 926-932.

56. Shimokawa $\mathrm{H}$. Hydrogen peroxide as an endothelium- derived hyperpolarizing factor. Pflugers Archiv 2010; 459: 915-922.

57. Gao YJ, Lee RM. Hydrogen peroxide induces a greater contraction in mesenteric arteries of spontaneously hypertensive rats through thromboxane $\mathrm{A}(2)$ production. $\mathrm{Br} \mathrm{J}$ Pharmacol 2001; 134: 1639-1646. 
58. Cogolludo A, Frazziano G, Cobeno L, et al. Role of reactive oxygen species in Kv channel inhibition and vasoconstriction induced by TP receptor activation in rat pulmonary arteries. Ann NY Acad Sci 2006; 1091: 41-51.

59. Garcia-Redondo AB, Briones AM, Beltran AE, Alonso MJ, Simonsen U, Salaices M. Hypertension increases contractile responses to hydrogen peroxide in resistance arteries through increased thromboxane $\mathrm{A} 2, \mathrm{Ca} 2+$, and superoxide anion levels. J Pharmacol Exp Ther 2009; 328: 19-27.

60. lida $Y$, Katusic ZS. Mechanisms of cerebral arterial relaxations to hydrogen peroxide. Stroke 2000; 31: 2224-2230.

61. Thakali K, Davenport L, Fink GD, Watts SW. Cyclooxygenase, p38 mitogen-activated protein kinase (MAPK), extracellular signal-regulated kinase MAPK, Rho kinase, and Src mediate hydrogen peroxide-induced contraction of rat thoracic aorta and vena cava. $\mathrm{J}$ Pharmacol Exp Ther 2007; 320: 236-243.

62. Chaplin NL, Amberg GC. Hydrogen peroxide mediates oxidant-dependent stimulation of arterial smooth muscle L- type calcium channels. Am J Physiol Cell Physiol 2012; 302: C1382-C1393.

63. Jin N, Rhoades RA. Activation of tyrosine kinases in $\mathrm{H} 2 \mathrm{O} 2-$ induced contraction in pulmonary artery. Am J Physiol 1997; 272: H2686-H2692.

64. Zembowicz A, Hatchett RJ, Jakubowski AM, Gryglewski RJ. Involvement of nitric oxide in the endothelium- dependent relaxation induced by hydrogen peroxide in the rabbit aorta. Br J Pharmacol 1993; 110: 151-158.

65. Leffler CW, Busija DW, Armstead WM, Mirro R. H2O2 effects on cerebral prostanoids and pial arteriolar diameter in piglets. Am J Physiol 1990; 258: H1382-H1387.

66. Neo BH, Kandhi S, Wolin MS. Roles for soluble guanylate cyclase and a thiol oxidation-elicited subunit dimerization of protein kinase $\mathrm{G}$ in pulmonary artery relaxation to hydrogen peroxide. Am J Physiol Heart Circul Physiol 2010; 299: H1235-H1241.

67. Miura H, Bosnjak JJ, Ning G, Saito T, Miura M, Gutterman DD. Role for hydrogen peroxide in flow-induced dilation of human coronary arterioles. Circ Res 2003; 92: e31e40.

68. Zoer B, Kessels L, Vereijken A, et al. Effects of prenatal hypoxia on pulmonary vascular reactivity in chickens prone to pulmonary hypertension. J Physiol Pharmacol 2009; 60: 119-130. 


\title{
Chapter 5
}

\section{Vasoactivity of the gasotransmitters hydrogen sulfide and carbon monoxide in the chicken ductus arteriosus}

\author{
Saskia van der Sterren, Pamela Kleikers, \\ Luc J.I. Zimmermann, Eduardo Villamor \\ Am J Physiol Regul Integr Comp Physiol 301: R1186-1198, 2011
}




\section{Abstract}

Besides nitric oxide (NO) and carbon monoxide $(\mathrm{CO})$, hydrogen sulfide $\left(\mathrm{H}_{2} \mathrm{~S}\right)$ is a third gaseous messenger that may play a role in controlling vascular tone and has been proposed to serve as an $\mathrm{O}_{2}$ sensor. However, whether $\mathrm{H}_{2} \mathrm{~S}$ is vasoactive in the ductus arteriosus (DA) has not yet been studied. We investigated, using wire myography, the mechanical responses in- duced by $\mathrm{Na}_{2} \mathrm{~S}(1 \mu \mathrm{M}-1 \mathrm{mM})$, which forms $\mathrm{H}_{2} \mathrm{~S}$ and $\mathrm{HS}-$ in solution, and by authentic $\mathrm{CO}$ $(0.1 \mu \mathrm{M}-0.1 \mathrm{mM})$ in DA rings from 19-day chicken embryos. $\mathrm{Na}_{2} \mathrm{~S}$ elicited a $100 \%$ relaxation $\left(\mathrm{pD}_{2} 4.02\right)$ of $21 \% \mathrm{O}_{2}$-contracted and a $50.3 \%$ relaxation of $62.5 \mathrm{mM} \mathrm{KCl}$-contracted $\mathrm{DA}$ rings. $\mathrm{Na}_{2} \mathrm{~S}$-induced relaxation was not affected by presence of the NO synthase inhibitor L-NAME, the soluble guanylate cyclase (sGC) inhibitor ODQ, or the $\mathrm{K}^{+}$channel inhibitors tetraethylammonium (TEA; nonselective), 4-aminopyridine (4-AP, Kv), glibenclamide (K $\mathrm{K}_{\mathrm{ATP}}$ ), iberiotoxin (BK $\mathrm{Ca}_{\mathrm{Ca}}$ ), TRAM-34 ( $\left.\mathrm{IK}_{\mathrm{Ca}}\right)$, and apamin $\left(\mathrm{SK}_{\mathrm{Ca}}\right)$. CO also relaxed $\mathrm{O}_{2}$-contracted (60.8\% relaxation) and $\mathrm{KCl}$-contracted $(18.6 \%$ relaxation) $\mathrm{DA}$ rings. $\mathrm{CO}$-induced relaxation was impaired by ODQ, TEA, and 4-AP (but not by L-NAME, glibenclamide, iberiotoxin, TRAM-34 or apamin), suggesting the involvement of $\mathrm{sGC}$ and $\mathrm{K}_{v}$ channel stimulation. The presence of inhibitors of $\mathrm{H}_{2} \mathrm{~S}$ or $\mathrm{CO}$ synthesis as well as the $\mathrm{H}_{2} \mathrm{~S}$ precursor L-cysteine or the $\mathrm{CO}$ precursor hemin did not significantly affect the response of the DA to changes in $\mathrm{O}_{2}$ tension. Endothelium-dependent and -independent relaxations were also unaffected. In conclusion, our results indicate that the gasotransmitters $\mathrm{H}_{2} \mathrm{~S}$ and $\mathrm{CO}$ are vasoactive in the chicken DA but they do not suggest an important role for endogenous $\mathrm{H}_{2} \mathrm{~S}$ or $\mathrm{CO}$ in the control of chicken ductal reactivity. 


\subsection{Introduction}

Nitric oxide synthesized from L-arginine by nitric oxide synthase (NOS) and carbon monoxide (CO) synthesized from heme by heme oxygenase $(\mathrm{HO})$ are well-known gaseous messengers that, among several other functions, play a pivotal role in the regulation of vascular tone (39). Recent studies indicate that another gas, hydrogen sulfide $\left(\mathrm{H}_{2} \mathrm{~S}\right)$, is also produced in substantial amounts in a variety of cells and exerts many physiological effects, suggesting its potential role as a regulatory mediator $(39,45,51)$. Two key enzymes in the transsulfuration pathway, cystathionine $\beta$-synthase (CBS) and cystathionine $\gamma$ lyase(CSE), have been consistently shown to produce $\mathrm{H}_{2} \mathrm{~S}$, using L-cysteine as substrate $(39,45,51,57)$. The expression of CBS is more abundant in liver and neuronal tissues, while CSE is the dominant $\mathrm{H}_{2} \mathrm{~S}$-generating enzyme in the cardiovascular system. A growing amount of research supports that by modulating vascular tone, promoting apoptosis of vascular smooth muscle cells, and inhibiting proliferation-associated vascular remodeling, $\mathrm{H}_{2} \mathrm{~S}$ participates in the regulation of both function and structure of the circulatory system $(39,41,45,49,51,57,69,70)$.

The ductus arteriosus (DA) is a large fetal shunt connecting the pulmonary artery to the aorta, allowing most of the right ventricular output to bypass the unexpanded lungs $(10,56)$. Although the cyclooxygenase pathway, with $\mathrm{PGE}_{2}$ as its major effector, is assigned a prime role in the active maintenance of fetal DA patency, the gasotransmitters $\mathrm{NO}$ and $\mathrm{CO}$ are viewed as additional effectors acquiring prominence under certain conditions (7). However, whether the third gaseous mediator, i.e., $\mathrm{H}_{2} \mathrm{~S}$, is also a modulator of DA tone has not been investigated so far.

The failure of the DA to close after birth is a neonatal complication, often associated with premature birth, which can negatively impact the outcome of preterm infants (56). Therefore, advancing our knowledge on the mechanisms that regulate vascular tone in the DA and its developmental biology may have direct clinical significance. Recently, the chicken embryo has emerged as a suitable model for the study of DA developmental biology (56). Our group and another laboratory have characterized the responsiveness of the chicken DA to several mediators that participate in the control of ductal tone in mammals, including $\mathrm{O} 2, \mathrm{NO}, \mathrm{CO}_{2}, \mathrm{PGs}$, sex hormones, and catecholamines (2- 4, 9, 20, 33, 34, 46, 61). Since the gasotransmitters $\mathrm{H}_{2} \mathrm{~S}$ and $\mathrm{CO}$ are important endogenous signaling molecules in numerous vascular beds, we hypothesized that they would be vasoactive also in the chicken DA. Moreover, since $\mathrm{H}_{2} \mathrm{~S}$ has been proposed as vascular $\mathrm{O}_{2}$ sensor, we hypothesized its role in the process 
of $\mathrm{O}_{2}$ sensing/signaling in the DA. To test these hypotheses, we analyzed, using wire myography, the mechanical responses induced by $\mathrm{Na}_{2}$, which forms $\mathrm{H}_{2} \mathrm{~S}$ and HS- in solution (26), and by authentic CO (62) in chicken DA rings. We also evaluated the effects of the precursors and inhibitors of the endogenous synthesis of either $\mathrm{H}_{2} \mathrm{~S}$ or $\mathrm{CO}$ on ductal reactivity and responsiveness to $\mathrm{O}_{2}$. The DA was compared with femoral and pulmonary arteries, which respond in disparate ways to changes in $\mathrm{O}_{2}$ tension $(2,20,53,72)$.

\subsection{Methods}

\subsubsection{Embryo incubation and vessel isolation}

All experimental procedures were carried out according to the regulations of the Dutch Law on Animal Experimentation and the European Directive for the Protection of Vertebrate Animals Used for Experimental and Other Scientific Purposes (86/609/EU) and were approved by the Committee on Animal Experimentation of the University of Maastricht. Fertilized eggs of White Leghorn chickens (Het Anker, Ochten, The Netherlands) were incubated at a temperature of $37.8^{\circ} \mathrm{C} / 45 \%$ air humidity and automatically rotated once every hour over an angle of 90 degrees (incubator model 25HS; Masalles Comercial, Spain). Embryos incubated for 15 and 19 days of the 21-day incubation period were studied. The majority of the experiments were performed in 19-day (noninternally pipped) embryos, while for the study of developmental changes, the 15-day and the 19-day vessels were compared. The experiments involving pulmonary arteries were performed in externally pipped 21-day embryos, because pulmonary arteries from 19-day embryos show, in general, weak vasomotor responses (63). On the experimental day, the embryos were taken out, immediately killed by decapitation, and a midline laparotomy and sternotomy were performed. With the aid of a dissecting microscope, the right and the left DA were carefully dissected free from surrounding tissue, severed distal to the origin in the right or left pulmonary artery and proximal to the insertion into the aorta, and divided in two segments referred to as pulmonary side and aortic side (PulmDA and AoDA, respectively). The boundary between pulmonary and aortic side was determined based on the marked differences in diameter observed along the chicken DA $(2,4)$. In some embryos, rings of the femoral and the caudomedial intrapulmonary arteries were also obtained, as previously described $(63,72)$. 


\subsubsection{Recording of arterial reactivity}

Two stainless steel wires (diameter $40 \mu \mathrm{m}$ ) were inserted into the lumen of the vessels, which were mounted as ring segments (mean length, $1.72 \mathrm{~mm}, \mathrm{SD}$ 0.31 ) between an isometric force transducer and a displacement device in a myograph (model 610M; Danish Myo Technology, Aarhus, Denmark). The myograph organ bath ( $5 \mathrm{ml}$ volume) was filled with Krebs-Ringer bicarbonate (KRB) buffer maintained at $39^{\circ} \mathrm{C}$. After an equilibration period of $30 \mathrm{~min}$, the vessels were distended to a resting tension corresponding to a transmural pressure of $10 \mathrm{mmHg}$ (15-day embryos) or $20 \mathrm{mmHg}$ (19-day and 21-day embryos). These pressures correspond to the mean arterial blood pressure reported in chicken embryos at the corresponding age (6) and elicit the highest contractile response to $\mathrm{KCl}$, as determined in previous experiments $(33,46)$. After $30 \mathrm{~min}$ of incubation at basal tone, a control contraction was elicited by raising the $\mathrm{K}^{+}$concentration of the buffer to $62.5 \mathrm{mM}$ (in exchange for $\mathrm{Na}^{+}$). During the first phase of mounting and stabilization, DA rings were maintained in $\mathrm{KRB}$ buffer aerated with $95 \% \mathrm{~N}_{2} / 5 \% \mathrm{CO}_{2}\left(\mathrm{Po}_{2} 2.48 \mathrm{kPa}\right.$, SD 0.34, $n=12$, measured with an $\mathrm{ABL} 510$ blood gas analyzer; Radiometer Copenhagen, Denmark). Afterward, the gas mixture was switched to $21 \% \mathrm{O}_{2} / 74 \% \mathrm{~N}_{2} / 5 \% \mathrm{CO}_{2}$ $\left(\mathrm{Po}_{2} 19.16 \mathrm{kPa}, \mathrm{SD} 1.15, n=12\right)$ to induce a normoxic contraction of the PulmDA $(2,3)$. The relaxations evoked by $\mathrm{H}_{2} \mathrm{~S}$ and $\mathrm{CO}$ were mainly studied during this normoxic contraction, but some experiments (see results, paragraph 5.3) were performed in vascular rings (DA, femoral, and pulmonary arteries) precontracted with $\mathrm{KCl}(62.5 \mathrm{mM})$ or phenylephrine $(10 \mu \mathrm{M})$ and aerated with $5 \% \mathrm{O}_{2} / 90 \% \mathrm{~N}_{2} / 5 \% \mathrm{CO}_{2}\left(\mathrm{Po}_{2} 6.96 \mathrm{kPa}, \mathrm{SD} 0.52, n=12\right)$ or with $95 \% \mathrm{~N}_{2} / 5 \%$ $\mathrm{CO}_{2}$. The latter gas mixture was also used to induce a direct hypoxic contraction in AoDA and pulmonary artery rings.

\subsubsection{Response of chicken DA to exogenous $\mathrm{H}_{2} \mathrm{~S}$ and $\mathrm{CO}$}

$\mathrm{Na}_{2} \mathrm{~S}(1 \mu \mathrm{M}-1 \mathrm{mM})$, which forms $\mathrm{H}_{2} \mathrm{~S}$ and $\mathrm{HS}-$ in solution, was used to analyze the response to $\mathrm{H}_{2} \mathrm{~S}$ because of its availability with a reduced amount of elemental sulfur impurities $(26,27) . \mathrm{Na}_{2} \mathrm{~S}$ was dissolved in deoxygenated HEPES buffer under $100 \% \mathrm{~N}_{2}$ and titration to $\mathrm{pH} 7.4$ with $\mathrm{HCl}$.

Concentration-response curves to $\mathrm{CO}(0.1 \mu \mathrm{M}-0.1 \mathrm{mM})$ were conducted by addition of increasing volumes of a HEPES buffer solution $(\mathrm{pH} 7.4)$ saturated with $\mathrm{CO}$, as previously described (62). The concentration of $\mathrm{CO}$ in the saturated solution was estimated from the solubility of $\mathrm{CO}$ in water at $25^{\circ} \mathrm{C}$ and $1 \mathrm{~atm}$ of 
pressure (62). We assumed that the loss of added CO from the HEPES solution at the time of measuring relaxation was negligible. Because this assumption was not strictly correct, actual concentrations of $\mathrm{CO}$ in the organ chamber might be somewhat lower than estimated (62).

To assess the mechanisms involved in the response of the chicken DA to the different vasoactive mediators, some experiments were performed in the presence of the following pharmacological tools: the NOS inhibitor NG-nitro-Larginine methyl ester (L-NAME; $0.1 \mathrm{mM}$ ), the soluble guanylate cyclase (sGC) inhibitor $1 \mathrm{H}$-[1,2,4]oxadiazolo[4,3-a]quinoxalin-1-one (ODQ, $10 \mu \mathrm{M})$, the nonselective $\mathrm{K}^{+}$channel inhibitor tetraethylammonium (TEA, $5 \mathrm{mM}$ ), the voltagegated $\mathrm{K}^{+}$channel $\left(\mathrm{K}_{\mathrm{V}}\right)$ inhibitor 4-aminopyridine (4-AP; $10 \mathrm{mM}$ ), the ATPsensitive $\mathrm{K}^{+}$channel (KATP) inhibitor glibenclamide $(10 \mu \mathrm{M})$, the largeconductance $\mathrm{Ca}^{2+}$-activated $\mathrm{K}^{+}$channel $\left(\mathrm{BK}_{\mathrm{Ca}}\right)$ inhibitor iberiotoxin $(100 \mathrm{nM})$, the intermediate-conductance $\mathrm{K}_{\mathrm{Ca}}\left(\mathrm{IK}_{\mathrm{Ca}}\right)$ channel inhibitor TRAM-34 $(1 \mu \mathrm{M})$, the small-conductance $\mathrm{K}_{\mathrm{Ca}}\left(\mathrm{SK}_{\mathrm{Ca}}\right)$ channel inhibitor apamin $(50 \mathrm{nM})$, the TP receptor antagonist SQ29548 $(10 \mu \mathrm{M})$, the dual endothelin (ET) receptor antagonist bosentan $(10 \mu \mathrm{M})$, the L-type $\mathrm{Ca}^{2+}$ channel blocker nifedipine (10 $\mu \mathrm{M}$ ), and the sarcoendoplasmic reticulum $\mathrm{Ca}^{2+-A T P a s e}$ inhibitor thapsigargin (2 $\mu \mathrm{M})$. Parallel control experiments were always carried out to correct for the possible effects of the different vehicles used to dissolve the drugs. In another group of experiments, the endothelium was removed by gentle rubbing of the vessel lumen with a horse tail, as previously described (4). The absence of a functional endothelium was verified by the failure of acetylcholine $(1 \mu \mathrm{M})$ to induce relaxation of the vascular tissues precontracted with phenylephrine (10 $\mu \mathrm{M})(4)$.

\subsubsection{Effects of endogenous $\mathrm{H}_{2} \mathrm{~S}$ and $\mathrm{CO}$ on $D A$ reactivity}

In this group of experiments, the effects of inhibiting or stimulating $\mathrm{H}_{2} \mathrm{~S}$ and $\mathrm{CO}$ synthesis on the response of the PulmDA to normoxia and hypoxia were examined. $\mathrm{O}_{2}$-induced contraction in the chicken $\mathrm{DA}$ is easily reversible when returning to hypoxia and highly reproducible in two consecutive challenges (20). Thus, each vessel was exposed twice to a cycle of hypoxia-normoxia-hypoxia and the second exposure was elicited in the presence of vehicle (control), the CSE-inhibitor D,L- propargylglycine (PPG; $1 \mathrm{mM}$ ), the CBS-inhibitor aminooxyacetate $\left(A O A ; 1 \mathrm{mM}\right.$ ), the substrate for endogenous $\mathrm{H}_{2} \mathrm{~S}$ production ( $\mathrm{L}-$ cysteine; $1 \mathrm{mM}$ ), the HO-inhibitor zinc protoporphyrin IX (ZnPP IX; $10 \mu \mathrm{M})$, or the substrate for endogenous $\mathrm{CO}$ production hemin $(10 \mu \mathrm{M})$. Experiments involving ZnPP or hemin were carried out in the dark (19). Due to the scarce information on the effects of the above mentioned compounds in chicken 
tissues, doses were selected based on their effects in mammalian tissues ( 7 , $19,52,65)$.

In previous studies, we demonstrated that acetylcholine (ACh) evoked an endothelium-dependent (NO-mediated) relaxation, whereas the NO donor sodium nitroprusside (SNP) evoked an endothelium- independent relaxation in the chicken DA (4). To analyze the possible role of $\mathrm{H}_{2} \mathrm{~S}$ and $\mathrm{CO}$ in these relaxations, concentration response curves to ACh (10 nM-10 $\mu \mathrm{M})$ and SNP (10 $\mathrm{nM}-0.1 \mathrm{mM}$ ) were constructed in $\mathrm{O}_{2}$-contracted $\mathrm{DA}$ rings in the absence or presence of PPG (1 mM), AOA (1 mM), L-cysteine (1 mM), or ZnPP IX (10 $\mu \mathrm{M})$.

\subsubsection{Drugs and solutions}

KRB buffer contained (in mmol/l): $118.5 \mathrm{NaCl}, 4.75 \mathrm{KCl}, 1.2 \mathrm{MgSO}_{4} \cdot 7 \mathrm{H}_{2} \mathrm{O}, 1.2$ $\mathrm{KH}_{2} \mathrm{PO}_{4}, 25.0 \mathrm{NaHCO}_{3}, 2.5 \mathrm{CaCl}_{2}, 5.5$ glucose. HEPES buffer contained (in $\mathrm{mmol} / \mathrm{l}): 142.9 \mathrm{NaCl}, 4.75 \mathrm{KCl}, 1.2 \mathrm{MgSO}_{4} \cdot 7 \mathrm{H}_{2} \mathrm{O}, 1.2 \mathrm{KH}_{2} \mathrm{PO}_{4}, 2.5 \mathrm{CaCl}_{2}, 5.5$ glucose, 15.0 HEPES. Solutions containing different concentrations of $\mathrm{K}^{+}$were prepared by replacing $\mathrm{NaCl}$ by an equimolar amount of $\mathrm{KCl}$. $\mathrm{CO}$ was obtained from Lindegas Benelux. Tetraethylammonium chloride, glibenclamide, and Lcysteine were obtained from Alexis Biochemicals (Lausen, Switzerland). SQ29548 was obtained from Cayman Chemical (Ann Arbor, MI). All other drugs were obtained from Sigma (St. Louis, MO). All drugs were initially dissolved in distilled deionized water, except ODQ, TRAM-34, ZnPP IX, hemin, and SQ29548, which were dissolved in DMSO, nifedipine, which was dissolved in ethanol and 4-AP, which was directly dissolved in KRB buffer.

\subsubsection{Data analysis}

Results are presented as the mean (SD) of measurements in the number of $(n)$ embryos. For clarity of figures, results are shown as means $\pm \mathrm{SE}$. Contractions are expressed in terms of active wall tension, calculated as the force divided by twice the length of the arterial segment $(\mathrm{mN} / \mathrm{mm})$. Relaxations are expressed as the percentage of reduction of the contraction induced by $21 \% \mathrm{O} 2, \mathrm{KCl}$ or phenylephrine. Sensitivity/potency (expressed as pD2 $=-\log \mathrm{EC}_{50}$ ) and efficacy (expressed as $E_{\max }$ ) were calculated by nonlinear regression analysis of the concentration-response curves. Differences between mean values were assessed by unpaired $t$-tests or one-way ANOVA, followed by post hoc Bonferroni $t$-test. Differences were considered statistically significant at $P<$ 0.05. All analyses were performed using a commercially available statistics package (Graph-Pad Prism version 5, GraphPad InStat version 3.00; GraphPad Soft- ware, San Diego, CA). 


\subsection{Results}

5.3.1 Response of chicken embryo vessels to contractile stimuli

In endothelium-intact PulmDA rings from 19-day chicken embryos, exposure to $21 \% \mathrm{O}_{2}$ evoked a mean contraction of $0.342 \mathrm{mN} / \mathrm{mm}$ (SD 0.07, $n=48$ ). $\mathrm{KCl}$ $\left(62.5 \mathrm{mM}\right.$, in the presence of $\left.5 \% \mathrm{O}_{2}\right)$ evoked a mean contraction of $0.121 \mathrm{mN} /$ $\mathrm{mm}$ (SD 0.04, $n=14$ ) in PulmDA rings from 15-day embryos, $0.462 \mathrm{mN} / \mathrm{mm}$ (SD 0.04, $n=18$ ) in PulmDA rings from 19-day embryos, $0.512 \mathrm{mN} / \mathrm{mm}$ (SD $0.16, n=12$ ) in AoDA rings from 19-day embryos, $1.423 \mathrm{mN} / \mathrm{mm}$ (SD 0.22, $n=$ 16) in femoral artery rings from 19-day embryos, and $0.543 \mathrm{mN} / \mathrm{mm}$ (SD 0.12, $n$ = 9) in pulmonary artery rings from 21-day embryos. $\mathrm{KCl}(62.5 \mathrm{mM}$, in the presence of $0 \% \mathrm{O}_{2}$ ) evoked a mean contraction of $0.503 \mathrm{mN} / \mathrm{mm}$ (SD $0.12, n=$ 6) in PulmDA rings from 19-day embryos. Finally, phenylephrine $(10 \mu \mathrm{M})$ evoked, in PulmDA rings from 19-day embryos, mean contractions of 0.587 $\mathrm{mN} / \mathrm{mm}(\mathrm{SD} 0.18, n=6)$ and $0.596 \mathrm{mN} / \mathrm{mm}(\mathrm{SD} 0.19, n=6)$ under $0 \%$ and $5 \%$ $\mathrm{O}_{2}$, respectively.
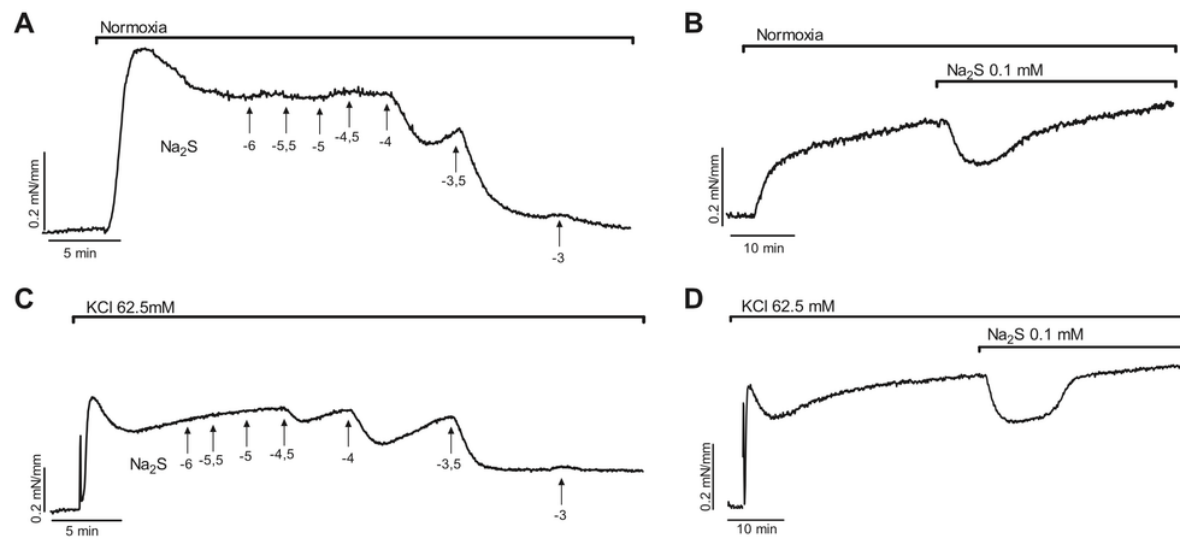

D

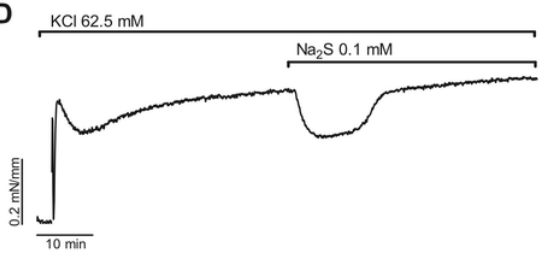

Fig. 5.1. Relaxant effects of $\mathrm{Na}_{2} \mathrm{~S}$ in chicken embryo ductus arteriosus (DA). Representative tracing of active wall tension vs. time showing the response of $2 \mathrm{O}_{2}$-contracted DA rings (19-day, pulmonary side; $A$ and $B$ ) and two $62.5 \mathrm{mM} \mathrm{KCl}$-contracted DA rings (19-day, pulmonary side; $C$ and $D$ ) to cumulative $\left(A\right.$ and $C$ ) or single $\left(B\right.$ and $D$ ) concentrations of $\mathrm{Na}_{2} \mathrm{~S}$. Values indicate log $\mathrm{M}$ $\left[\mathrm{Na}_{2} \mathrm{~S}\right]$. KCl-induced contractions were performed under $5 \% \mathrm{O}_{2}$. The deflections observed in the traces $C$ and $D$ (at the beginning of $\mathrm{KCl}$-induced contractions) correspond to the replacement of the buffer. 


\subsubsection{Response to $\mathrm{Na}_{2} \mathrm{~S}$}

$\mathrm{Na}_{2} \mathrm{~S}$ relaxed $\mathrm{O}_{2}$-contracted PulmDA rings (19-day) in a dose-dependent manner (Figures $5.1 A$ and $5.2 A ; \mathrm{pD}_{2} 4.02$, SD $0.12, n=13$ ). When the PulmDA rings (19-day) were precontracted with $\mathrm{KCl}(62.5 \mathrm{mM}$ in the presence of $0 \%$ or $5 \% \mathrm{O}_{2}$ ), $\mathrm{Na}_{2} \mathrm{~S}$ also evoked a concentration-dependent relaxation (Figs. $5.1 \mathrm{C}$ and $5.2 A)$ with similar potency $\left(0 \% \mathrm{O}_{2}: \mathrm{pD} 23.97, \mathrm{SD} 0.38, n=6 ; 5 \% \mathrm{O}_{2}: \mathrm{pD}_{2}\right.$ 4.16, SD $0.42, n=9$ ) but lower efficacy than the observed in $\mathrm{O}_{2}$-contracted rings $\left(\mathrm{E}_{\max } \mathrm{KCl}\right.$-contracted $0 \% \mathrm{O}_{2}: 40.96 \%$, SD 27.2; $\mathrm{E}_{\max } \mathrm{KCl}$-contracted $5 \%$ $\mathrm{O}_{2}: 50.34 \%$, SD 27.2; $\mathrm{E}_{\max } \mathrm{O}_{2}$-contracted: $100.71 \%$, SD 18.4; $P<0.01$ vs. $\mathrm{KCl}-$ contracted in the presence of $0 \%$ or $5 \% \mathrm{O}_{2}$ ). When PulmDA rings (19-day) were precontracted with phenylephrine $\left(10 \mu \mathrm{M}\right.$, in the presence of $0 \%$ or $\left.5 \% \mathrm{O}_{2}\right)$, $\mathrm{Na}_{2} \mathrm{~S}$ also evoked a concentration- dependent relaxation (Fig. 5.2A) with similar potency $\left(0 \% \mathrm{O}_{2}: \mathrm{pD}_{2} 4.48\right.$, SD $0.44, n=6 ; 5 \% \mathrm{O}_{2}: \mathrm{pD}_{2} 4.51$, SD $\left.0.41, n=6\right)$ and efficacy as the ones observed in $\mathrm{O}_{2}$-contracted rings. As can be observed in Fig. 5.1, $A$ and $C$, the relaxations evoked by the lowest effective concentrations $(0.03-0.1 \mathrm{mM})$ of $\mathrm{Na}_{2} \mathrm{~S}$ were transient and followed by a progressive return of the tone toward the precontraction level. To assess whether this phenomenon was due to a biphasic effect (i.e., relaxation followed by contraction), we analyzed the time pattern of a more prolonged (>30 $\mathrm{min}$ ) exposure to $\mathrm{Na}_{2} \mathrm{~S}(0.1 \mathrm{mM}$, Figs. 5.1, $B$ and $D)$. The level of tension reached after $>30 \mathrm{~min}$ of exposure to $\mathrm{Na}_{2} \mathrm{~S}$ was $0.373 \mathrm{mN} / \mathrm{mm}(\mathrm{SD} 0.14, n=6)$ in the $\mathrm{O}_{2}$-contracted PulmDA rings and $0.493 \mathrm{mN} / \mathrm{mm}(\mathrm{SD} \mathrm{0.16,n=6)}$ in the $\mathrm{KCl}$ contracted vessels. This level of tension was not significantly different from the one observed in time-control experiments $\left(\mathrm{O}_{2}\right.$-contracted: $0.358 \mathrm{mN} / \mathrm{mm}, \mathrm{SD}$ $0.18, n=4 ; \mathrm{KCl}$-contracted: $0.511, \mathrm{SD} 0.21, n=4)$. This suggests that $\mathrm{Na}_{2} \mathrm{~S}$ did not evoke an actual contraction and that the concentration of $\mathrm{H}_{2} \mathrm{~S}$ might have been reduced due to constant bubbling of $\mathrm{O}_{2}, \mathrm{CO}_{2}$, and $\mathrm{N}_{2}$ in the organ bath, inducing the progressive return of active wall tension towards the precontraction line. $\mathrm{KCl}$-contracted PulmDA rings from 15-day embryos were relaxed by $\mathrm{Na}_{2} \mathrm{~S}$ (Fig. $5.2 B)$ with similar potency $\left(\mathrm{pD}_{2} 4.24, \mathrm{SD} 0.14, n=6\right)$ and efficacy $\left(E_{\max }\right.$ $34.25 \%$, SD 13.6) as observed for 19-day PulmDA rings. $\mathrm{Na}_{2} \mathrm{~S}$ also relaxed $\mathrm{KCl}$-contracted 19-day AoDA rings $\left(\mathrm{pD}_{2} 3.98, \mathrm{SD} 0.18, n=9\right)$, 19-day femoral artery rings $\left(\mathrm{pD}_{2} 4.23, \mathrm{SD} 0.34, n=8\right)$, and 21-day pulmonary artery rings $\left(\mathrm{pD}_{2}\right.$ 4.29, SD $0.28, n=9$ ) with similar potency and efficacy as observed for 19-day PulmDA rings (Fig. 5.2B).

The efficacy (Fig. $5.2 \mathrm{C}$ ) and the potency of $\mathrm{Na}_{2} \mathrm{~S}$ to relax $\mathrm{O}_{2}$-contracted PulmDA rings (19-day) were not affected by endothelium removal (pD2 3.89, SD $0.22, n=6$ ), or by the presence of the NOS inhibitor L-NAME (pD2 4.11, SD $0.13, \mathrm{n}=10)$, the $\mathrm{sGC}$ inhibitor $\mathrm{ODQ}\left(\mathrm{pD}_{2} 3.92, \mathrm{SD} 0.17, n=8\right)$, the 

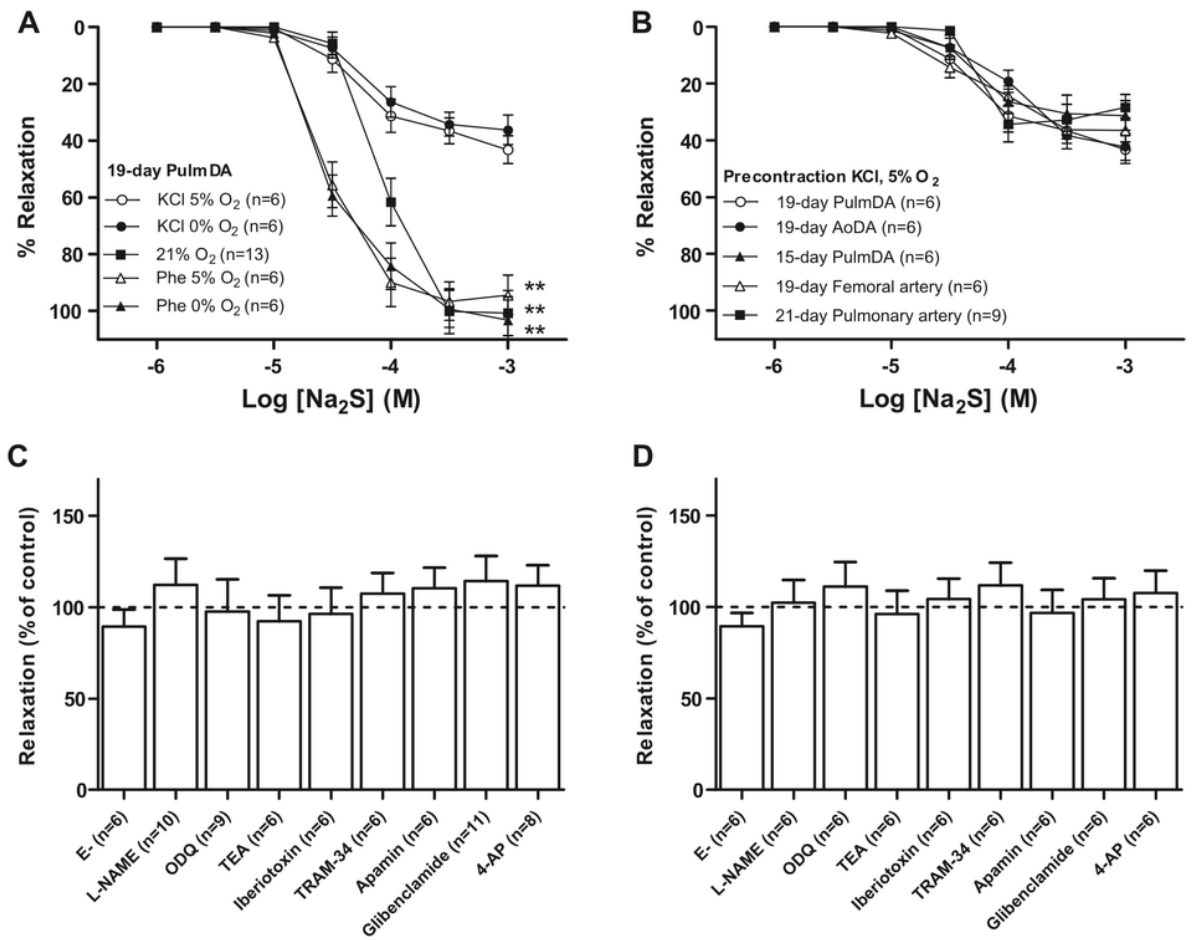

Fig. 5.2. A: mean $\pm \mathrm{SE}$ cumulative dose-response curves for $\mathrm{Na}_{2} \mathrm{~S}$ in $21 \% \mathrm{O}_{2^{-}}, 62.5 \mathrm{mM} \mathrm{KCl}$, or 1 $\mu \mathrm{M}$ phenylephrine (Phe)-contracted 19-day DA (pulmonary side) rings. $\mathrm{KCl}$ and Phe contractions were performed under $0 \%$ and $5 \% \mathrm{O}_{2} .{ }^{* *} \mathrm{P}<0.01$ for difference in $\mathrm{E}_{\max }$ compared with $\mathrm{KCl}$ contracted. $B$ : mean $\pm \mathrm{SE}$ cumulative dose-response curves for $\mathrm{Na}_{2} \mathrm{~S}$ in $\mathrm{KCl}$-contracted DA (PulmDA, pulmonary side; AoDA, aortic side), femoral and pulmonary artery rings from 15-day, 19day, and 21-day chicken embryos. Contractions were performed under $5 \% \mathrm{O}_{2} . \mathrm{C}$ and $\mathrm{D}$ : maximal relaxation (mean $\pm \mathrm{SE}$ ) evoked by $\mathrm{Na}_{2} \mathrm{~S}(1 \mu \mathrm{M}-1 \mathrm{mM})$ in $\mathrm{O}_{2}$-contracted $(C)$ and $10 \mu \mathrm{M}$ Phecontracted $(D)$ PulmDA rings (19-day) after endothelium removal (E-) or in the presence of the nitric oxide (NO) synthase inhibitor $N G$-nitro-L-arginine methyl ester (L-NAME; $0.1 \mathrm{mM}$ ), the soluble guanylate cyclase inhibitor $\mathrm{ODQ}(10 \mu \mathrm{M})$, the nonselective $\mathrm{K}^{+}$channel inhibitor tetraethylammonium (TEA; $5 \mathrm{mM}$ ), the voltage-gated $\mathrm{K}^{+}$channel $\left(\mathrm{K}_{\mathrm{V}}\right)$ inhibitor 4-aminopyridine (4-AP; $10 \mathrm{mM}$ ), the ATPsensitive $\mathrm{K}^{+}$channel (KATP) inhibitor glibenclamide $(10 \mu \mathrm{M})$, the large-conductance $\mathrm{Ca}^{2+}$-activated $\mathrm{K}$ + channel (BKCa) inhibitor iberiotoxin (100 nM), the intermediate-conductance $\mathrm{KCa}\left(\mathrm{IK}_{\mathrm{Ca}}\right)$ channel inhibitor TRAM-34 $(1 \mu \mathrm{M})$, or the small-conductance $\mathrm{KCa}\left(\mathrm{SK}_{\mathrm{Ca}}\right)$ channel inhibitor apamin $(50 \mathrm{nM})$. The experiments involving Phe $(D)$ were performed under $0 \% \mathrm{O}_{2}\left(\mathrm{Po}_{2}, \sim 2.5 \mathrm{kPa}\right)$. The data are expressed as \% response observed in the control (endothelium intact, vehicle-treated) group.

nonselective $\mathrm{K}^{+}$channel inhibitor TEA $\left(\mathrm{pD}_{2} 4.07\right.$, SD $\left.0.12, n=6\right)$, the $\mathrm{K}_{\mathrm{V}}$ channel inhibitor 4-AP $\left(\mathrm{pD}_{2} 4.01, \mathrm{SD} 0.14, n=8\right)$, the KATP channel inhibitor glibenclamide $\left(\mathrm{pD}_{2} 4.08, \mathrm{SD} 0.23, n=11\right)$, the $\mathrm{BK}_{\mathrm{Ca}}$ channel inhibitor iberiotoxin $\left(\mathrm{pD}_{2} 4.14\right.$, SD 0.16, $\left.n=6\right)$, the $\mathrm{IK}_{\mathrm{Ca}}$ channel inhibitor TRAM-34 $\left(\mathrm{pD}_{2} 4.02\right.$, SD 
0.18, $n=6)$, or the SK $\mathrm{Ca}_{\mathrm{a}}$ channel inhibitor apamin $\left(\mathrm{pD}_{2} 3.96\right.$, $\left.\mathrm{SD} 0.18, n=6\right)$. A significant contraction $(P<0.05$ vs. the respective vehicle) was observed following the 20- to 30-min incubation with L-NAME $(0.101 \mathrm{mN} / \mathrm{mm}, \mathrm{SD} 0.08)$, ODQ (0.132 $\mathrm{mN} / \mathrm{mm}$, SD 0.09), and 4-AP $(0.124 \mathrm{mN} / \mathrm{mm}, \mathrm{SD} 0.05)$, but not following incubation with TEA, iberiotoxin, TRAM-34, or apamin. The subsequent contraction induced by $\mathrm{O}_{2}$ was significantly reduced $(0.246 \mathrm{mN} /$ $\mathrm{mm}$, SD 0.09, $n=8, P<0.05$ vs. control) when the PulmDA rings were incubated with 4-AP. The other experimental conditions did not affect $\mathrm{O}_{2}$ induced contraction in PulmDA rings. Finally, the efficacy (Fig. 5.2D) and the potency (data not shown) of $\mathrm{Na}_{2} \mathrm{~S}$ to relax 19-day PulmDA rings precontracted with phenylephrine $\left(10 \mu \mathrm{M}\right.$, in the presence of $\left.0 \% \mathrm{O}_{2}\right)$ were not significantly affected by any of the above-described experimental conditions.

\subsubsection{Role of $\mathrm{H}_{2} \mathrm{~S}$ in oxygen sensing in chicken embryo vessels}

To investigate whether endogenous $\mathrm{H}_{2} \mathrm{~S}$ affected the response to $\mathrm{O}_{2}$ of chicken DA, PulmDA rings (19-day) were exposed twice to a cycle of hypoxia-normoxiahypoxia, and the second exposure was elicited in the presence of vehicle or the substrate for endogenous $\mathrm{H}_{2} \mathrm{~S}$ production L-cysteine, the CSE inhibitor PPG, or the CBS inhibitor AOA. As shown in Fig. 5.3, A-D, L-cysteine, PPG, and AOA did not affect the subsequent contraction evoked by normoxia or the relaxation evoked by hypoxia. A significant contraction $(P<0.05$ vs. the respective vehicle) was observed following the incubation with PPG $(0.176 \mathrm{mN} / \mathrm{mm}$, SD 0.12 ) and L-cysteine $(0.210 \mathrm{mN} / \mathrm{mm}, \mathrm{SD} 0.07)$ (see Fig. $5.4, A-C$ ), but not following incubation with AOA.

In a separate group of experiments, we examined the mechanisms involved in L-cysteine-induced contraction of 19-day PulmDA rings. As shown in Fig. 5.3E, L-cysteine-induced contraction was not significantly affected by $\mathrm{O}_{2}$ tension, endothelium removal, or by the presence of PPG, AOA, the TP receptor antagonist SQ29548, or the dual ET receptor antagonist bosentan. In contrast, when 19-day PulmDA rings were incubated in a $\mathrm{Ca}^{2+}$-free medium (containing 1 mM EGTA) or with the L-type $\mathrm{Ca}^{2+}$ channel blocker nifedipine, the contraction evoked by L-cysteine was significantly impaired. The remaining component of the L-cysteine-induced tonic contraction in the absence of $\mathrm{Ca}^{2+}\left(0 \mathrm{mM} \mathrm{Ca}^{2+}+1\right.$ mM EGTA) was almost completely inhibited by pretreatment with thapsigargin, which depletes internal $\mathrm{Ca}^{2+}$ stores (44). 
A

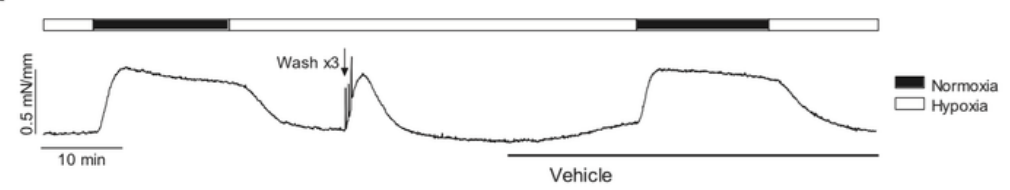

B

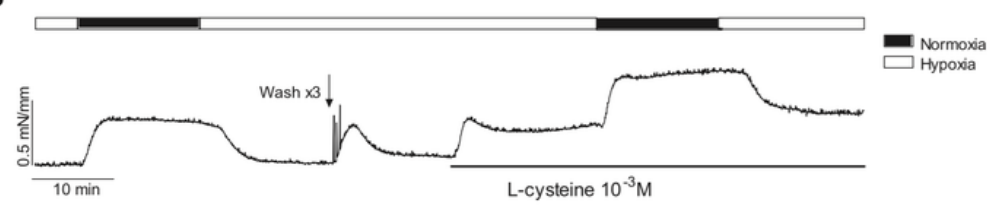

C
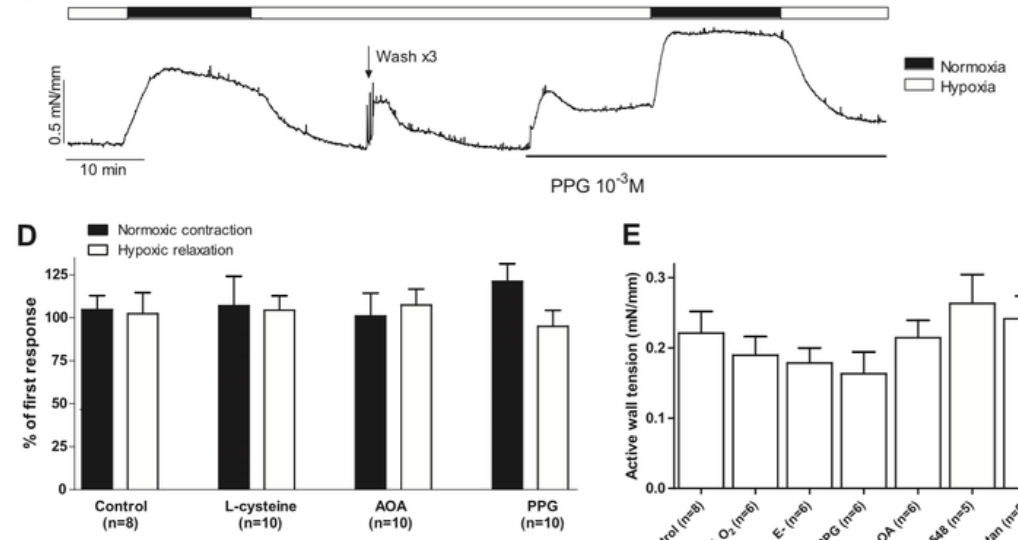

$\mathrm{E}$

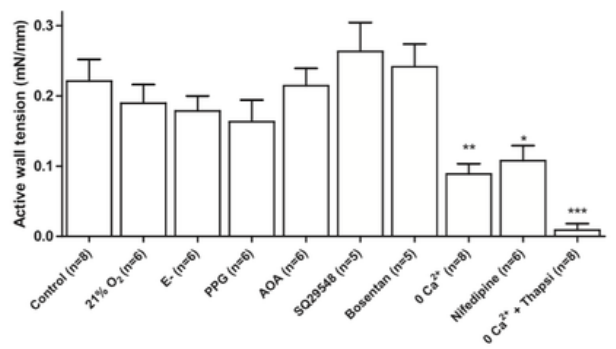

Fig. 5.3. Lack of role for endogenous $\mathrm{H}_{2} \mathrm{~S}$ in the response to normoxia/hypoxia of chicken $D A$. $A-D$ : DA rings (19-day, pulmonary side) were exposed twice to a cycle of hypoxia-normoxia-hypoxia and the second exposure was elicited in the presence of vehicle, the substrate for endogenous $\mathrm{H}_{2} \mathrm{~S}$ production L-cysteine $(1 \mathrm{mM})$, the cystathionine $\gamma$-lyase inhibitor $\mathrm{D}, \mathrm{L}-$-propargylglycine (PPG; $1 \mathrm{mM})$, or the cystathionine $\beta$-synthase inhibitor amino-oxyacetate (AOA; $1 \mathrm{mM})$. Normoxic contraction and hypoxic relaxation in $D$ are expressed as \% first maximal response in the same vessel. $E$ : mean \pm SE active wall tension developed by DA rings (19-day, pulmonary side) in response to L-cysteine (1 $\mathrm{mM}$ ). Except when otherwise stated, all the experiments were performed under $0 \% \mathrm{O}_{2}$. L-Cysteineinduced contraction was not significantly affected by $\mathrm{O}_{2}$ tension, endothelium removal (E-), or by the presence of PPG, AOA, the TP receptor antagonist SQ29548 $(10 \mu \mathrm{M})$, or the dual endothelin receptor antagonist bosentan $(10 \mu \mathrm{M})$. In contrast, when vessels were incubated in a $\mathrm{Ca}^{2+-f r e e}$ medium (containing $1 \mathrm{mM}$ EGTA, $0 \mathrm{Ca}^{2+}$ ) or with the L-type $\mathrm{Ca}^{2+}$ channel blocker nifedipine (10 $\mu \mathrm{M})$, the contraction evoked by L-cysteine was significantly impaired. The remaining component of the L-cysteine-induced tonic contraction in the absence of $\mathrm{Ca}^{2+}$ was almost completely inhibited by pretreatment with the sarcoendoplasmic reticulum $\mathrm{Ca}^{2+}$-ATPase inhibitor thapsigargin $(2 \mu \mathrm{M}) .{ }^{*} P<$ $0.05,{ }^{\star \star} P<0.01,{ }^{\star \star \star} P<0.001$ vs. control. 
A

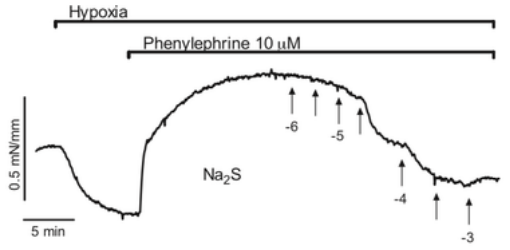

C

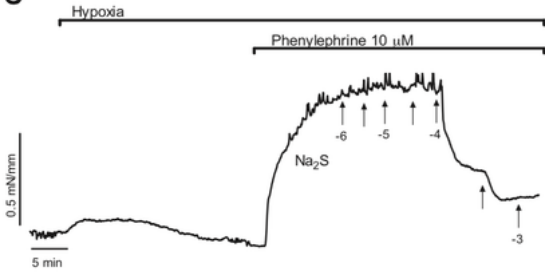

E

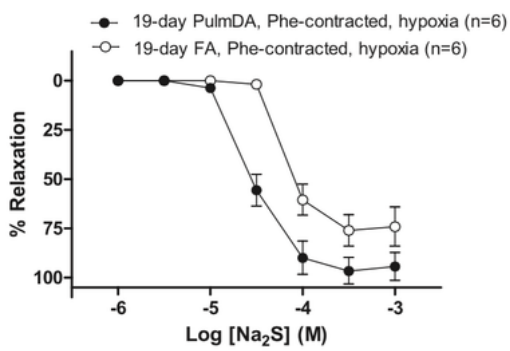

B

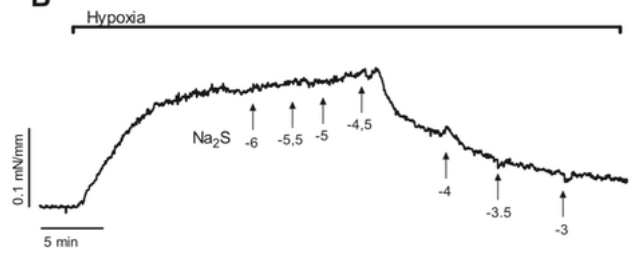

D

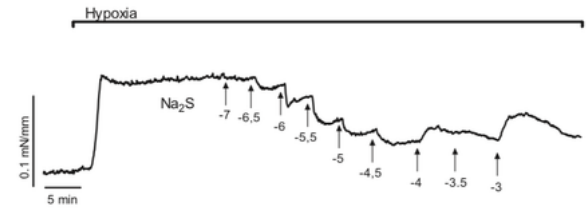

$\mathbf{F}$

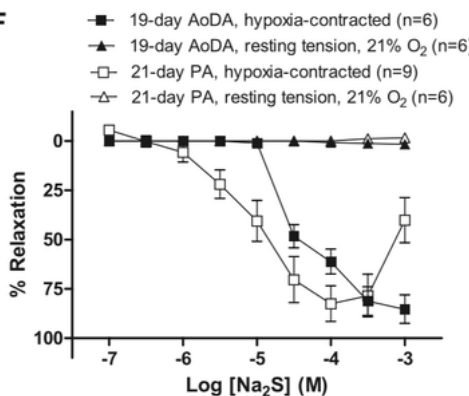

Fig. 5.4. Relaxant effects of $\mathrm{Na}_{2} \mathrm{~S}$ in chicken embryo vessels under hypoxic conditions $\left(\mathrm{Po}_{2}, \sim 2.5\right.$ $\mathrm{kPa})$. Hypoxia evoked a relaxation in the pulmonary side (PulmDA; $A$ ) and a tonic contraction in the aortic side (AoDA; $B)$ of the DA and in the pulmonary artery (PA; $D)$. In the femoral artery (FA;C), hypoxia induced a transient contraction. The PulmDA and the femoral artery rings were subsequently precontracted with phenylephrine $(10 \mu \mathrm{M})$ to evaluate the response to $\mathrm{Na}_{2} \mathrm{~S}$. In the experiments involving AoDA and PA rings, the precontraction was the one evoked by hypoxia itself. $E-F$ : mean $\pm S E$ cumulative dose-response curves for $\mathrm{Na}_{2} \mathrm{~S}$ in chicken embryo vessels exposed to the experimental conditions described above. The responses to $\mathrm{Na}_{2} \mathrm{~S}$ of nonprecontracted (resting tension) AoDA and PA rings (bubbled with $21 \% \mathrm{O}_{2}$ ) are also depicted $(F)$.

Olson et al. (52) reported that $\mathrm{H}_{2} \mathrm{~S}$ and hypoxia produced the same mechanical response in vessels from at least one species in every vertebrate class and that the effects of $\mathrm{H}_{2} \mathrm{~S}$ and hypoxia were competitive. As described above, hypoxia and $\mathrm{H}_{2} \mathrm{~S}$ evoked relaxation in the chicken PulmDA rings. However, this effect did not appear to be competitive since $\mathrm{Na}_{2} \mathrm{~S}$ relaxed $\mathrm{KCl}$ - and phenylephrinecontracted PulmDA rings under hypoxic conditions (Figs. 5.2A and 5.4, $A$ and $E$ ). To assess whether hypoxia and $\mathrm{H}_{2} \mathrm{~S}$ evoked similar responses in other chicken embryo vessels, we studied AoDA, femoral, and pulmonary artery 
rings. Hypoxia evoked a tonic contraction in the AoDA $(0.162 \mathrm{mN} / \mathrm{mm}$, SD 0.11, $n=6)$ and the pulmonary artery rings $(0.109 \mathrm{mN} / \mathrm{mm}, \mathrm{SD} 0.10, n=9)$ (Fig. 5.4), as previously described $(2,20,73)$. This hypoxic contraction was relaxed by $\mathrm{Na}_{2} \mathrm{~S}$ in a concentration-dependent manner $\left(\mathrm{pD}_{2}\right.$ AoDA: 4.41, SD 0.32, $n=8$; $\mathrm{pD}_{2}$ pulmonary artery: $5.1, \mathrm{SD} 0.41, n=6$ ). Interestingly, high concentrations ( $\geq$ $0.1 \mathrm{mM}$ ) of $\mathrm{Na}_{2} \mathrm{~S}$ evoked a transient contraction in the hypoxia-contracted pulmonary artery rings (Figs. 5.4, $D$ and $F$ ). In contrast, in the quiescent (nonprecontracted) pulmonary artery and AoDA rings (exposed to $21 \% \mathrm{O}_{2}$ ), $\mathrm{Na}_{2} \mathrm{~S}$ did not elicit significant mechanical effects (Fig. 5.4F). In the quiescent femoral arteries, hypoxia induced a transient contraction (Fig. 5.4C), as previously described (53). The tone of the vessels was subsequently increased with phenylephrine $(10 \mu \mathrm{M})$ to evaluate the response to $\mathrm{Na}_{2} \mathrm{~S}$ under hypoxic conditions. $\mathrm{Na}_{2} \mathrm{~S}$ relaxed the hypoxic, phenylephrine-contracted femoral artery rings in a concentration-dependent manner $\left(\mathrm{pD}_{2}: 4.11\right.$, SD 0.36, $n=6$, Fig. $\left.4 E\right)$.

A

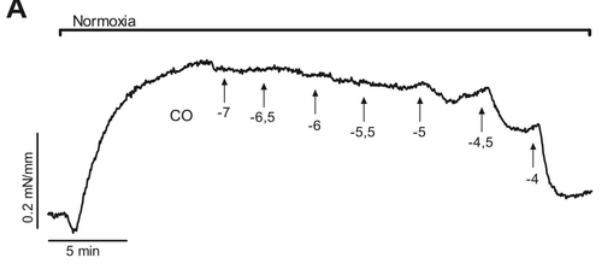

C

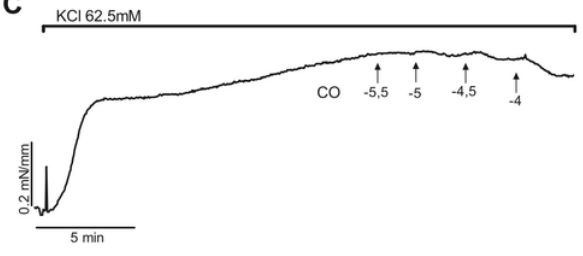

B

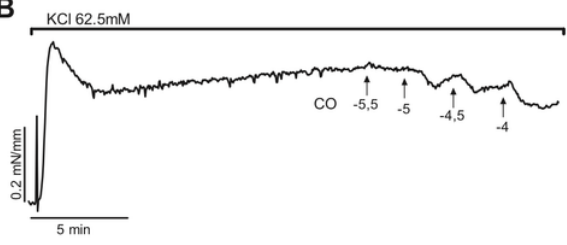

D

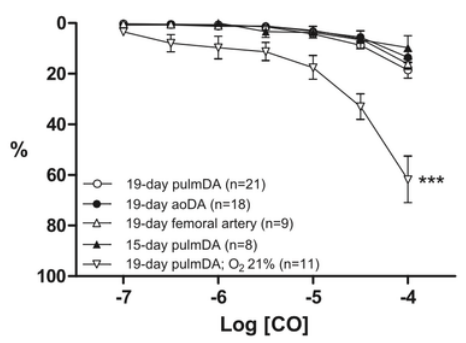

Fig. 5.5. Relaxant effects of authentic $C O$ in chicken embryo vessels. $A-C$ : representative tracing of active wall tension vs. time showing the response of an $\mathrm{O}_{2}$-contracted DA ring (19-day, pulmonary side) $(A)$, a $62.5 \mathrm{mM} \mathrm{KCl}$-contracted DA ring (19-day, pulmonary side) $(B)$, and a $62.5 \mathrm{mM} \mathrm{KCl}$ contracted DA ring (19-day, aortic side) $(C)$ to increasing concentrations of $\mathrm{CO} . \mathrm{KCl}$ contractions were performed under $5 \% \mathrm{O}_{2}$. Values indicate log $\mathrm{M}[\mathrm{CO}]$. $D$ : mean $\pm \mathrm{SE}$ cumulative dose-response curves for $\mathrm{CO}$ in $\mathrm{O}_{2-}$ or $\mathrm{KCl}$-contracted DA (PulmDA, pulmonary side; AoDA, aortic side) and femoral artery rings from 15 - and 19-day chicken embryos. ${ }^{* \star *} P<0.001$ for difference in maximal relaxation when compared with $\mathrm{KCl}$-contracted/19-day/PulmDA. 


\subsubsection{Response to CO}

As shown in Fig. 5.5, $A$ and $D, \mathrm{CO}$ relaxed $\mathrm{O}_{2}$-contracted PulmDA rings (19day) in a dose-dependent manner. However, a maximum relaxation was not achieved with the highest concentration of CO tested $(0.1 \mathrm{mM})$, and a $\mathrm{pD}_{2}$ value could therefore not be calculated. When the PulmDA rings (19-day) were contracted with $\mathrm{KCl}\left(62.5 \mathrm{mM}\right.$ in the presence of $\left.5 \% \mathrm{O}_{2}\right), \mathrm{CO}$ also evoked a concentration-dependent relaxation (Figs. 5.5, $B$ and $D$ ), which was significantly lower than the relaxation observed when the vessels were precontracted with $21 \% \mathrm{O}_{2}$. For example, the relaxation evoked by $0.1 \mathrm{mM} \mathrm{CO}$ was $61.86 \%$ (SD $30.57, n=11$ ) in $\mathrm{O}_{2}$-contracted PulmDA rings and $18.55 \%$ (SD 14.74, $n=21$ ) in $\mathrm{KCl}$-contracted vessels $\left(P<0.001\right.$ vs. $\mathrm{O}_{2}$-contracted). CO also relaxed $\mathrm{KCl}$ contracted AoDA (Figs. 5.5, $C$ and $D$ ) and femoral artery rings from 19-day embryos and PulmDA rings from 15-day embryos with similar efficacy as the one observed in PulmDA rings from 19-day embryos (Fig. 5.5D).

As shown in Fig. 5.6A, the relaxation induced by $\mathrm{CO}(0.1 \mathrm{mM})$ was markedly impaired in the presence of the SGC inhibitor ODQ, the nonselective $\mathrm{K}^{+}$channel inhibitor TEA or the $\mathrm{K}_{v}$ channel inhibitor 4-AP. Endothelium removal or the presence of the $\mathrm{K}_{\text {ATP }}$ channel inhibitor glibenclamide, the $\mathrm{BK}_{\mathrm{Ca}}$ channel inhibitor iberiotoxin, the $\mathrm{IK}_{\mathrm{Ca}}$ channel inhibitor TRAM-34, or the $\mathrm{SK}_{\mathrm{Ca}}$ channel inhibitor apamin did not significantly affect CO-induced relaxation (Fig. 5.6A). To investigate whether the inhibition of $\mathrm{K}_{\mathrm{v}}$ channels also affected other relaxations mediated through the sGC/cGMP pathway, we analyzed the effects of 4-AP on the relaxation induced by the $\mathrm{NO}$ donor $\mathrm{SNP}$ in $\mathrm{O}_{2}$-contracted PulmDA rings. As shown in Fig. 5.6B, the presence of 4-AP significantly impaired the potency $\left(\mathrm{pD}_{2} 5.16\right.$, SD $0.21, n=7$ vs. $\left.5.58, \mathrm{SD} 0.30, n=6 ; P<0.05\right)$ and the efficacy $\left(E_{\max } 29.35 \%\right.$, SD 13.78 vs. $67.13 \%$, SD $\left.21.86, P<0.01\right)$ of SNP.

In another group of experiments, we analyzed the effects of the HO-inhibitor zinc protoporphyrin (ZnPP IX; $10 \mu \mathrm{M})$, and the substrate for endogenous $\mathrm{CO}$ production hemin $(10 \mu \mathrm{M})$ on the response of the PulmDA to normoxia. Incubation for 20-30 min of PulmDA rings (19-day) with ZnPP IX did not affect basal tone or $\mathrm{O}_{2}$-induced contraction (data not shown). A more prolonged incubation $(4 \mathrm{~h})$ was performed with hemin $(10 \mu \mathrm{M})$. During this prolonged incubation a transient increase in the tone of the PulmDA rings was observed, but this transient increase in wall tension was also observed in the vessels incubated for $4 \mathrm{~h}$ in the presence of vehicle (DMSO). In the hemin group the second response to normoxia was $30.61 \%$ (SD 37.6, $n=5$ ) of the first contraction. However, a similar percentage of the first contraction was observed in the control group $(25.22 \%, \mathrm{SD} 22.97, n=4)$. 

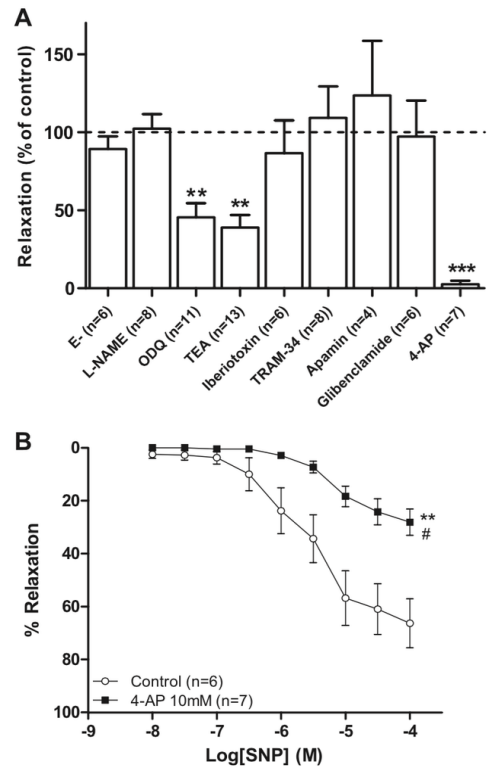

Fig. 5.6. $A$ : maximal relaxation (mean $\pm \mathrm{SE}$ ) evoked by authentic $\mathrm{CO}(0.1 \mu \mathrm{M}-0.1 \mathrm{mM})$ in $\mathrm{O}_{2-}$ contracted DA rings (pulmonary side, 19-day) after endothelium removal (E-) or in the presence of the NO synthase inhibitor L-NAME $(0.1 \mathrm{mM})$, the soluble guanylate cyclase inhibitor ODQ $(10 \mu \mathrm{M})$, the nonselective $\mathrm{K}^{+}$channel inhibitor tetraethylammonium (TEA; $5 \mathrm{mM}$ ), the voltage-gated $\mathrm{K}^{+}$ channel $\left(K_{\mathrm{V}}\right)$ inhibitor 4-aminopyridine (4-AP; $10 \mathrm{mM}$ ), the ATP-sensitive $\mathrm{K}^{+}$channel $\left(\mathrm{K}_{\text {ATP }}\right)$ inhibitor glibenclamide $(10 \mu \mathrm{M})$, the large-conductance $\mathrm{Ca}^{2+-}$ activated $\mathrm{K}^{+}$channel $\left(\mathrm{BK}_{\mathrm{Ca}}\right)$ inhibitor iberiotoxin $(100 \mathrm{nM})$, the intermediate- conductance $\mathrm{K}_{\mathrm{Ca}}\left(\mathrm{IK}_{\mathrm{Ca}}\right)$ channel inhibitor TRAM-34 $(1 \mu \mathrm{M})$, or the smallconductance $\mathrm{K}_{\mathrm{Ca}}\left(\mathrm{SK}_{\mathrm{Ca}}\right)$ channel inhibitor apamin $(50 \mathrm{nM})$. The data are expressed as $\%$ response observed in the control (endothelium intact, vehicle-treated) group. ${ }^{* \star} P<0.01,{ }^{* \star *} P<0.001$ vs. control. $B$ : mean $\pm \mathrm{SE}$ cumulative dose-response curves for the NO donor sodium nitroprusside (SNP) in $\mathrm{O}_{2}$-contracted DA rings (19-day, pulmonary side) in the absence (control) or the presence of 4-AP (10 mM). ${ }^{\star *} P<0.01$, for difference (vs. control) in $E_{\max } ; \# P<0.05$ for difference (vs. control) in $\mathrm{pD}_{2}$.

5.3.5 Role of endogenous $\mathrm{H} 2 \mathrm{~S}$ and $\mathrm{CO}$ in the relaxations evoked by ACh and SNP

In this group of experiments, we analyzed the effects of L-cysteine, PPG, AOA, and ZnPP IX on the relaxations evoked by ACh and SNP in $\mathrm{O}_{2}$-contracted PulmDA rings (19-day). As shown in Fig. 5.7, no significant effects were observed. In contrast, the NOS inhibitor L-NAME $(0.1 \mathrm{mM})$ impaired AChinduced relaxation $\left(\mathrm{pD}_{2}\right.$ control: $6.95, \mathrm{SD} 0.27, n=9 ; \mathrm{pD}_{2} \mathrm{~L}-\mathrm{NAME}: 6.47, \mathrm{SD}$ $0.28, n=8 ; P<0.05$, Fig. $7 C$ ). The presence of ZnPP IX did not produce a further effect on the inhibition elicited by L-NAME $\left(\mathrm{pD}_{2} \mathrm{ZnPP} I \mathrm{IX}+\mathrm{L}-\mathrm{NAME}: 6.39\right.$, SD 0.27, $n=8$ ). 

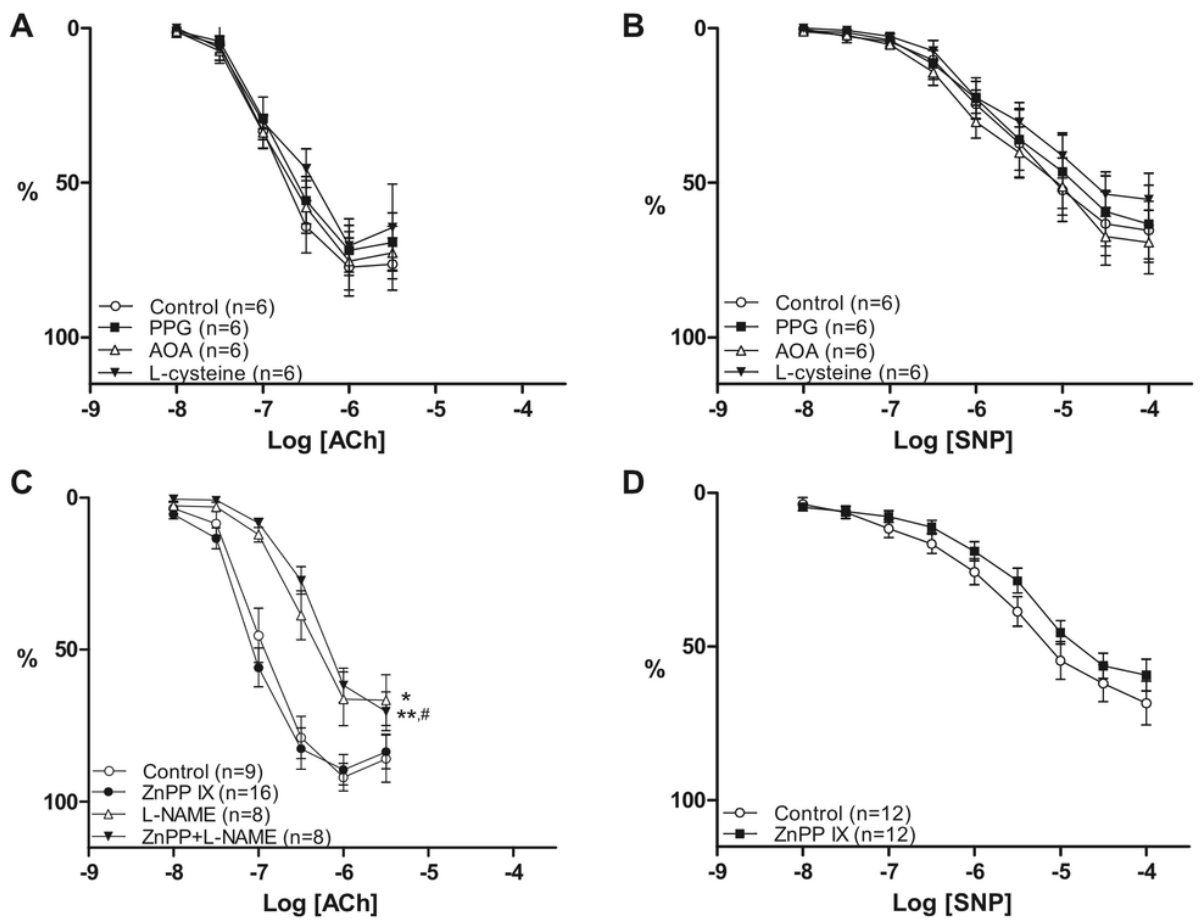

Fig. 5.7. Mean $\pm \mathrm{SE}$ cumulative dose-response curves for acetylcholine (ACh; $A$ and $C$ ) and sodium nitroprusside (SNP; $B$ and $D$ ) in $\mathrm{O}_{2}$-contracted DA rings (19-day, pulmonary side, endothelium intact) in the absence (control) or presence of L-cysteine ( $1 \mathrm{mM}, \mathrm{H}_{2} \mathrm{~S}$ substrate) D,L-propargylglycine (PPG; $1 \mathrm{mM}$, cystathionine $\gamma$-lyase inhibitor), amino-oxyacetate (AOA; $1 \mathrm{mM}$, cystathionine $\beta$ synthase inhibitor), zinc protoporphyrin IX (ZnPP IX; $10 \mu \mathrm{M}$, heme oxygenase inhibitor), and LNAME (0.1 mM, NO synthase inhibitor). ${ }^{*} P<0.05,{ }^{\star \star} P 0.01$ for difference (vs. control) in $\mathrm{pD}_{2}$. $\# P<$ 0.05 for difference (vs. ZnPP IX) in $\mathrm{pD}_{2}$.

\subsection{Discussion}

Besides $\mathrm{NO}$ and $\mathrm{CO}, \mathrm{H}_{2} \mathrm{~S}$ is a third gaseous autocrine/ paracrine messenger, which has recently been shown to play important physiological roles $(39,45$, $50,51,57)$. The effects of $\mathrm{H}_{2} \mathrm{~S}$ have been investigated in numerous mammalian and nonmammalian blood vessels but not in the DA. In the present work, using isolated DA rings from chicken embryos, we show that $\mathrm{Na}_{2} \mathrm{~S}$, which forms $\mathrm{H}_{2} \mathrm{~S}$ and $\mathrm{HS}^{-}$in solution, induces a concentration-dependent relaxation of this vessel. Although we could not elucidate the mechanisms underlying this relaxation, the pathway mainly involved in mammalian vessels (i.e., $\mathrm{K}_{\mathrm{ATP}}$ channel activation) does not appear to play a role in $\mathrm{H}_{2} \mathrm{~S}$-induced relaxation of 
the chicken DA. On the other hand, exogenous CO relaxed the chicken DA by a pathway that involves $S G C$ and $\mathrm{Kv}_{v}$ channel activation. The presence of precursors/inhibitors of $\mathrm{H}_{2} \mathrm{~S}$ and $\mathrm{CO}$ synthesis did not significantly affect the response of the chicken DA to normoxia/ hypoxia and did not affect endothelium-dependent or -inde- pendent relaxation. Therefore, our results indicate that the gasotransmitters $\mathrm{H}_{2} \mathrm{~S}$ and $\mathrm{CO}$ are vasoactive in the chicken DA but they do not suggest an important role for endogenous $\mathrm{H}_{2} \mathrm{~S}$ or $\mathrm{CO}$ in the control of chicken ductal reactivity.

\subsection{1 $\mathrm{H}_{2} \mathrm{~S}$-induced relaxation of chicken $D A$}

$\mathrm{H}_{2} \mathrm{~S}$ relaxed chicken $\mathrm{DA}$ rings with similar potency $\left(\mathrm{pD}_{2} \sim 4\right)$ as the one reported, for example, in rat aorta $\left(\mathrm{pD}_{2}\right.$ 3.86) (69), or rat mesenteric artery $\left(\mathrm{pD}_{2}\right.$ 3.98) (15). These concentrations of $\mathrm{H}_{2} \mathrm{~S}$ have been considered as physiologically relevant by numerous investigators but it should be noted that recent studies employing different analytical techniques have not verified micromolar concentrations of $\mathrm{H}_{2} \mathrm{~S}$ in blood or tissues (see Ref. 50 for review). The exhaustive study of Dombkowski et al. (29) demonstrated that $\mathrm{H}_{2} \mathrm{~S}$ (in the highmicromolar range) has vasoactive properties in at least one species from each class of vertebrates. Interestingly, they show that $\mathrm{H}_{2} \mathrm{~S}$ induces either vascular relaxation or contraction and that these disparate responses may occur in different vascular beds within a single species or even in the same vessel at different levels of $\mathrm{H}_{2} \mathrm{~S}$ exposure (29). When they analyzed the response to $\mathrm{H}_{2} \mathrm{~S}$ in avian blood vessels (Pekin duck aorta and pulmonary arteries), they observed that $\mathrm{H}_{2} \mathrm{~S}$ was mainly contractile in either unstimulated or precontracted aorta, whereas in the pulmonary artery, $\mathrm{H}_{2} \mathrm{~S}$ produced multiphasic responses (29). In contrast, we observed that in chicken embryo vessels $\mathrm{H}_{2} \mathrm{~S}$ is mainly a relaxing agent. The information about the effects of $\mathrm{H}_{2} \mathrm{~S}$ in human vascular smooth muscle is limited, but $\mathrm{H}_{2} \mathrm{~S}$-induced relaxation has been demonstrated in the internal mammary artery (65) and the corpus cavernosum (22). However, low concentrations of $\mathrm{H}_{2} \mathrm{~S}(50 \mu \mathrm{M})$ evoked a contraction in the human mammary artery that appeared to be related to the inactivation of NO (65). As discussed below, our experimental data do not support the presence of an interaction between $\mathrm{NO}$ and $\mathrm{H}_{2} \mathrm{~S}$ in chicken embryo DA.

$\mathrm{Na}_{2} \mathrm{~S}$ was less efficacious in relaxing chicken DA rings contracted with $62.5 \mathrm{mM}$ $\mathrm{K}+$ than it was in vessels contracted with $\mathrm{O}_{2}$ or phenylnephrine, suggesting the involvement of $\mathrm{K}^{+}$channels in the relaxation. The currently available data indicate that $\mathrm{H}_{2} \mathrm{~S}$ relaxes mammalian blood vessels mostly by opening $\mathrm{K}_{\text {ATP }}$ channels in smooth muscle cells (45). The K KTP channel antagonist 
glibenclamide attenuated $\mathrm{H}_{2} \mathrm{~S}$-induced relaxation in several mammalian and nonmammalian vessels, such as rat thoracic aorta $(41,59,70)$, rat mesenteric arteries (15), human internal mammary artery (65), and trout efferent branchial artery (28). In addition, patch-clamp studies have demonstrated that $\mathrm{H}_{2} \mathrm{~S}$ increases $\mathrm{K}_{\text {ATP-dependent current }}$ and induces hyperpolarization in mammalian vascular smooth muscle cells $(59,70)$. However, glibenclamide failed to affect the relaxing effect of $\mathrm{H}_{2} \mathrm{~S}$ in mouse aorta (37) and rat coronary artery (14). In the latter vessel, $\mathrm{H}_{2} \mathrm{~S}$-induced relaxation was impaired by the presence of the Kv channel blocker 4-AP (14). In the chicken DA, neither glibenclamide, nor 4AP or inhibitors of other major known $\mathrm{K}^{+}$channels altered $\mathrm{H}_{2} \mathrm{~S}$-induced relaxation. This result is in correspondence with the lack of effect of $\mathrm{K}^{+}$channel blockers on $\mathrm{H}_{2} \mathrm{~S}$-induced relaxation in mammalian bronchial (36) and gastrointestinal smooth muscles $(24,60)$ and suggests that the mechanisms underlying $\mathrm{H}_{2} \mathrm{~S}$-mediated relaxation are strongly tissue- and speciesdependent.

It has been suggested that the reduced efficacy of $\mathrm{H}_{2} \mathrm{~S}$ to relax smooth muscles precontracted with $\mathrm{KCl}$ could be the result of the interference between $\mathrm{Cl}^{-}$and $\mathrm{HS}^{-}$(27). $\mathrm{HS}^{-}$, which at physiological pH accounts for $\sim 80 \%$ of the total $\mathrm{H}_{2} \mathrm{~S}+$ $\mathrm{HS}^{-}$, may interfere with a $\mathrm{Cl}^{-}$channel or transporter and this interference would be diminished when transmembrane $\mathrm{Cl}-$ gradients are changed by using high concentrations of $\mathrm{KCl}$ (27). However, this could not explain the difference in efficacy we found since, in our experiments, solutions containing different concentrations of $\mathrm{K}^{+}$were prepared by replacing $\mathrm{NaCl}$ by an equimolar amount of $\mathrm{KCl}$. Therefore, the transmembrane $\mathrm{Cl}^{-}$gradient was not changed and could not play a role in $\mathrm{H}_{2} \mathrm{~S}$ induced relaxation.

A crosstalk between $\mathrm{H}_{2} \mathrm{~S}$ and $\mathrm{NO}$ has been suggested in some vascular tissues, but the mechanisms are still not clear (64). $\mathrm{H}_{2} \mathrm{~S}$ has been shown to either enhance (69) or attenuate (35) the relaxant effect of $\mathrm{NO}$ in the rat aorta. It has been reported that $\mathrm{H}_{2} \mathrm{~S}$ may impair NO-induced relaxation through the inhibition of endothelial NOS (eNOS) (37), the scavenging of NO with the formation of a nitrosothiol compound (66), the decrease in the sensitivity of the cGMP pathway to NO (64), or the modification of $\mathrm{K}_{\mathrm{Ca}}$ channels to decrease their sensitivity to NO (64). On the other hand, NO may increase the expression of CSE or the cellular uptake of cysteine (64). Our present results in the chicken embryo DA do not suggest an interaction between $\mathrm{H}_{2} \mathrm{~S}$ and $\mathrm{NO}$ in this vessel, since inhibition of NOS and SGC did not affect $\mathrm{H}_{2} \mathrm{~S}$-induced relaxation. Furthermore, the presence of inhibitors of $\mathrm{H}_{2} \mathrm{~S}$ synthesis or the $\mathrm{H}_{2} \mathrm{~S}$ precursor Lcysteine did not affect the relaxation evoked by ACh or SNP. 
Unlike $\mathrm{NO}$ or $\mathrm{CO}$, specific receptors for $\mathrm{H}_{2} \mathrm{~S}$ have not been identified, and putative molecular targets appear to be both tissue and species dependent (42). Therefore, other possible mechanisms involved in the smooth muscle effects of $\mathrm{H}_{2} \mathrm{~S}$, such as changes in intracellular $\mathrm{pH}$ (38), production of reactive oxygen species (48), downregulation of CAMP (41), activation of adenylate cyclase (48), inhibition of phosphodiesterase activity (12), activation of phospholipase $\mathrm{A}_{2}$ (25), activation of myosin light chain phosphatase (23), or sulfhydration of actin (47) remain to be explored in chicken embryo vessels.

\subsection{2 $\mathrm{H}_{2} \mathrm{~S}$ as $\mathrm{O}_{2}$ sensor in the chicken $D A$}

Olson et al. (49) recently proposed that $\mathrm{H}_{2} \mathrm{~S}$ is an $\mathrm{O}_{2}$ sensor in vascular smooth muscle. This hypothesis is based on their observations that 1) $\mathrm{H}_{2} \mathrm{~S}$ and hypoxia produce the same mechanical response in vessels from at least one species in every vertebrate class; 2) the effects of $\mathrm{H}_{2} \mathrm{~S}$ and hypoxia are competitive; and 3) blood vessels enzymatically generate $\mathrm{H}_{2} \mathrm{~S}$ and inhibitors of $\mathrm{H}_{2} \mathrm{~S}$ synthesis inhibit hypoxic responses, whereas the $\mathrm{H}_{2} \mathrm{~S}$ precursor L-cysteine augments it. The DA is exquisitely sensitive to $\mathrm{O}_{2}(56)$, but the role of $\mathrm{H}_{2} \mathrm{~S}$ in its $\mathrm{O}_{2}$ responsiveness had not yet been investigated. The present study was performed in the chicken DA, a vessel that is the result of the fusion of two vessels with different embryological origin, morphology, and functionality. The PulmDA consists almost exclusively of neural crest-derived cells, shows the structure of a muscular artery, and responds to $\mathrm{O}_{2}$ with contraction (and to hypoxia with relaxation), whereas the AoDA is of mesodermal origin, shows the morphology of an elastic artery, relaxes in response to normoxia, and contracts in response to hypoxia $(2,9,10,20,34,61)$. Previous studies have shown the involvement of the mitochondrial electron transport chain as sensor, $\mathrm{H}_{2} \mathrm{O}_{2}$ as mediator, and $\mathrm{K}_{V}$ channels and Rho kinase as effectors of $\mathrm{O}_{2}$-induced contraction in the chicken PulmDA $(20,34)$. This indicates the presence of a common mechanism for $\mathrm{O}_{2}$ sensing/ signaling in mammalian and nonmammalian DA (56). The $\mathrm{O}_{2}$-induced relaxation of the AoDA, on the contrary, appears to be dependent on the NO/cGMP pathway mediated by endothelial cells (34).

In agreement with the results of Olson et al. $(49,52)$, we observed that $\mathrm{H}_{2} \mathrm{~S}$ and hypoxia produce the same mechanical response (i.e., relaxation) in the PulmDA. However, the response to $\mathrm{H}_{2} \mathrm{~S}$ and hypoxia was not competitive because PulmDA rings, when precontracted with $\mathrm{KCl}$ or phenylephrine under hypoxic conditions, still show $\mathrm{Na}_{2} \mathrm{~S}$-evoked relaxation. Moreover, $\mathrm{Na}_{2} \mathrm{~S}$ was 
also a relaxing agent in the immature 15-day PulmDA, which is not sensitive to the effects of normoxia/hypoxia $(2,20,33)$. In addition, neither hypoxic relaxation nor normoxic contraction of the 19-day PulmDA was affected by the presence of precursors/inhibitors of $\mathrm{H}_{2} \mathrm{~S}$ synthesis. It should be noted that the enzyme inhibitors used (AOA and PPG) lack specificity and are often poorly absorbed by intact tissues (57). What is more, to the best of our knowledge, there are no data available on the inhibitory effects of AOA and PPG in chicken tissues, and the doses that we used were based on studies in mammalian tissues. Despite these limitations, the present study suggests that $\mathrm{H}_{2} \mathrm{~S}$ is not involved in $\mathrm{O}_{2}$ sensing/signal transduction in the PulmDA.

In an effort to clarify the possible role of $\mathrm{H}_{2} \mathrm{~S}$ in $\mathrm{O}_{2}$ sensing in chicken embryo vasculature, we decided to further investigate the effects of $\mathrm{Na}_{2} \mathrm{~S}$ in two vessels, the AoDA and the pulmonary artery, which respond to hypoxia with contraction, and one vessel, the femoral artery, which (when precontracted) responds to hypoxia with relaxation $(2,20,53,72)$. We observed that in these vessels, under hypoxic conditions, $\mathrm{Na}_{2} \mathrm{~S}$ evoked a concentration-dependent relaxation (Fig. 4). Moreover, while hypoxia induced a tonic contraction in quiescent (nonprecontracted) AoDA and pulmonary artery rings, $\mathrm{Na}_{2} \mathrm{~S}$ did not elicit significant mechanical effects. Therefore, the ability of exogenous $\mathrm{H}_{2} \mathrm{~S}$ to mimic hypoxic responses was not found in the chicken embryo vessels that respond to hypoxia with contraction. The only partial exception to this pattern was the hypoxia-contracted pulmonary artery, which, although relaxed in response to low concentrations of $\mathrm{Na}_{2} \mathrm{~S}$, was contracted by higher $(>0.1 \mathrm{mM})$ concentrations. This observation is in agreement with that by Olson et al. (52) who demonstrated that $\mathrm{H}_{2} \mathrm{~S}$ evoked two dose-dependent effects in bovine pulmonary arteries. In these vessels, $\mathrm{H}_{2} \mathrm{~S}$ produced a dose-dependent relaxation between $10 \mathrm{nM}$ and $10 \mu \mathrm{M}$, whereas concentrations $>10 \mu \mathrm{M}$ produced a dose-dependent contraction. They hypothesize that much of the $\mathrm{H}_{2} \mathrm{~S}$ produced by the pulmonary vascular smooth muscle cells during normoxia will be oxidized and the resultant low $\mathrm{H}_{2} \mathrm{~S}$ concentration dilates the vessels and reduces vascular resistance. In contrast, during hypoxia, the concentration of $\mathrm{H}_{2} \mathrm{~S}$ will increase and result in vasoconstriction. However, this leads back to the question of whether the pulmonary vascular smooth muscle cells are able to produce the large amount of $\mathrm{H}_{2} \mathrm{~S}$ that may be required to induce vasoconstriction. Nevertheless, as Olson (51) has recently stressed, to date no study has identified any stimulus for $\mathrm{H}_{2} \mathrm{~S}$ production in cells in real time and under physiological conditions, other than showing an inverse relationship between $\mathrm{H}_{2} \mathrm{~S}$ production and $\mathrm{Po}_{2}$. The demonstration of this relationship in chicken embryo vessels warrants further investigation. 
L-Cysteine, the precursor of $\mathrm{H}_{2} \mathrm{~S}$, evoked a tonic contraction in the 19-day PulmDA rings. This contraction was endothelium- independent and was significantly inhibited either by omission of extracellular $\mathrm{Ca}^{2+}$ or by inclusion of the L-type $\mathrm{Ca}^{2+}$ channel blocker nifedipine. The remaining component of the Lcysteine-induced tonic contraction in the absence of $\mathrm{Ca}^{2+}$ was almost completely inhibited by pretreatment with thapsigargin, which depletes internal $\mathrm{Ca}^{2+}$ stores (44). Taken together, these findings suggest that L-cysteine elicits contraction of PulmDA rings by promoting $\mathrm{Ca}^{2+}$ influx via nifedipine-sensitive $\mathrm{Ca}^{2+}$ channels and intracellular $\mathrm{Ca}^{2+}$ release from the sarcoendoplasmic reticulum. Our results are consistent with the data obtained in both smooth and striated muscle, which indicate that $\mathrm{L}$-cysteine is an important modulator of $\mathrm{Ca}^{2+}$ homeostasis $(13,21,54)$. We also observed that neither the CSE inhibitor PPG nor the CBS inhibitor AOA significantly affected L-cysteine-induced contraction of PulmDA rings. This, together with the opposite effects of $\mathrm{H}_{2} \mathrm{~S}$ (relaxation) and L-cysteine (contraction) in this preparation, suggests that the contractile effect of L-cysteine was not related to $\mathrm{H}_{2} \mathrm{~S}$ production. In contrast to our findings, several studies in mammalian vessels demonstrated $\mathrm{H}_{2} \mathrm{~S}$-mediated relaxation to be elicited by L-cysteine $(5,15)$.

\subsubsection{CO-induced relaxation of chicken DA}

The endogenous production of $\mathrm{CO}$ occurs through the activity of HOs, enzymes that catalyze the degradation of heme to $\mathrm{CO}$, iron, and biliverdin. Three isoforms of $\mathrm{HO}$ have been identified: the highly inducible $\mathrm{HO}-1$ and the constitutively present $\mathrm{HO}-2$ and $\mathrm{HO}-3$ (11). $\mathrm{HO}-3$ is not enzymatically active in heme degradation, although it may function as a heme-sensing or -binding protein (11). CO participates in the regulation of vascular tone, smooth muscle proliferation and platelet aggregation. These effects of $\mathrm{CO}$ are mediated via multiple pathways, including activation of $\mathrm{SGC}$, activation of $\mathrm{K}^{+}$channels, and interference with the cytochrome P450 (CYP450)-based mono-oxygenase reaction, limiting the synthesis of ET-1 $(11,18,67)$. CO-induced relaxation has been described in pulmonary and systemic vessels as well as in the DA from several mammalian species $(1,7,16-19,32,62)$. Dombkowski et al. (30) demonstrated that the CO-releasing molecule CORM-3 relaxed sea lamprey and rainbow trout systemic arteries, suggesting that $\mathrm{CO}$ vasoactivity has been well conserved throughout vertebrate evolution. To our knowledge, the effects of exogenous $\mathrm{CO}$ in avian vessels had not been analyzed before. In the present work we show that the chicken embryo DA and femoral arteries are relaxed by exogenous $\mathrm{CO}$. The relaxant potency and efficacy of $\mathrm{CO}$ in these chicken vessels were comparable to the ones reported in mammalian vessels 
$(1,62)$.

The response of the mammalian DA to exogenous CO has been studied in the guinea pig and in the lamb $(16-19,32)$. In 1971, Fay (32) reported CO-induced relaxation of $\mathrm{O}_{2}$-contracted guinea pig DA rings. This finding was not confirmed by Coceani et al. (17) but they demonstrated CO-induced relaxation as well as $\mathrm{HO}-1$ and $\mathrm{HO}-2$ expression in endothelial and smooth muscle cells of the lamb DA $(17,18)$. Interestingly, they showed that CO-induced relaxation in the lamb DA is not mediated through a significant stimulation of $\mathrm{SGC}$ but through inhibition of the functional complex CYP450/ET-1 (18). In contrast, we observed that in the chicken DA CO-induced relaxation involves SGC and $\mathrm{K}_{v}$ channel stimulation.

The sGC/cGMP system is commonly regarded as main messenger for $\mathrm{CO}$ in smooth muscle cells (16). Accordingly, we observed that CO-induced relaxation in the chicken DA was markedly impaired in the presence of the SGC inhibitor ODQ. However, as mentioned above, CO can induce relaxation by acting on different targets including $\mathrm{K}^{+}$channels $(8,68)$. The dependence of $\mathrm{CO}$-induced relaxation of chicken $\mathrm{DA}$ on transmembrane $\mathrm{K}^{+}$gradient suggests that $\mathrm{K}^{+}$efflux through $\mathrm{K}^{+}$channels is involved in the response. Furthermore, 4-AP, which blocks $\mathrm{Kv}_{v}$ channels, significantly inhibited CO-induced relaxation. Electrophysiological and pharmacomechanical studies in a variety of mammalian vascular tissues have pointed to a pivotal role of $\mathrm{K}_{v}$ channels in sGC/cGMPmediated relaxation $(58,71)$. To analyze whether in the chicken DA the involvement of $\mathrm{K}_{v}$ channels was a particularity of $\mathrm{CO}$-induced relaxation, we tested the effects of the NO donor SNP in the presence of 4-AP. Previously, we demonstrated that SNP induced a SGC-dependent relaxation of chicken DA (4). Herein, we observed that 4-AP inhibited the relaxation evoked by SNP. This suggests that activation of $\mathrm{K}_{\mathrm{v}}$ channels occurs downstream from sGC activation. Therefore, a plausible mechanism for $\mathrm{CO}$-induced relaxation in the chicken DA would be sGC/cGMP-mediated activation of $\mathrm{K}_{v}$ channels rather than direct $\mathrm{CO}$-mediated channel regulation.

In the mouse DA, inhibition of $\mathrm{HO}$ with $\mathrm{ZnPP}$ induces a contraction that is curtailed in eNOS-deleted fetuses, indicating a crucial role for $\mathrm{NO}$ in $\mathrm{CO}$ mediated ductal tone (7). Moreover, the endothelium-dependent relaxation evoked by bradykinin in the mouse DA was suppressed by either L-NAME or ZnPP. This has also been found in the lamb DA (19) and suggests that, in these vessels, $\mathrm{NO}$ and $\mathrm{CO}$ act sequentially in mediating bradykinin-induced relaxation. In the chicken DA, bradykinin is not vasoactive (4) but ACh evokes an endothelium-dependent relaxation that is partially impaired by L- NAME (4). We observed that incubation with ZnPP did not induce a significant contraction 
of the chicken DA and did not affect ACh-induced relaxation either in the absence or presence of L-NAME. Accordingly, we previously demonstrated the lack of effect of $\mathrm{ZnPP}$ in ACh-induced relaxation of chicken embryo pulmonary arteries, suggesting an absent role for endogenous production of $\mathrm{CO}$ in chicken embryo vessels (63). However, $\mathrm{HO}$ is expressed in the chicken $(31,55)$ and Leo et al. (40) demonstrated a partial role for endogenous $\mathrm{CO}$ in the endothelium-derived relaxation evoked by ACh in carotid arteries from 6- to 8week-old chicken. This indicates the possibility of developmental and/or tissuespecific differences in the role of $\mathrm{CO}$ in the chicken vasculature.

\subsubsection{Perspectives and significance}

Of the many gases with biological roles, $\mathrm{O}_{2}$ is the most relevant with regard to the physiological regulation of the DA, but others, such as reactive $\mathrm{O}_{2}$ species, $\mathrm{CO}_{2}$, NO, and $\mathrm{CO}$ are also signaling molecules in this vessel. The present study shows, for the first time, the vasoactive properties of $\mathrm{H}_{2} \mathrm{~S}$ in the chicken DA. There are still numerous questions remaining before $\mathrm{H}_{2} \mathrm{~S}$ can be accepted as a biologically relevant signaling molecule $(42,51)$ and, therefore, our findings warrant further confirmation in the DA from other species. Blood vessels belong to a specialized homeostatic $\mathrm{O}_{2}$-sensing system, which responds rapidly to moderate changes in $\mathrm{O}_{2}$ tension (49). This has been extensively studied in mammals where hypoxia contracts pulmonary vessels, relaxes systemic vessels, and relaxes the DA, whereas normoxia evokes the opposite effect. The search for an evolutionary-conserved, ubiquitous $\mathrm{O}_{2}$ sensing mechanism represents an important and exciting area of research but the identity of the $\mathrm{O}_{2}$ sensor(s) is still elusive. Hypoxic constriction of pulmonary (73) and chorioallantoic arteries and the aortic side of the DA $(2,9,20,34,61)$, as well as hypoxic relaxation of femoral arteries $(53,72)$ and the pulmonary side of the DA $(2,9,20,34,61)$ are present in the chicken embryo vasculature that represents an interesting model to challenge the putative ubiquity of such vascular $\mathrm{O}_{2}$ sensing/signaling mediators like $\mathrm{H}_{2} \mathrm{~S}$. Finally, as the response to $\mathrm{H}_{2} \mathrm{~S}$ is strongly tissue- and species-dependent (29); future studies, comparing vessels from embryonic/fetal and adult animals, will elucidate whether the response to the gasotransmitter is also age-dependent. 


\section{References}

1. Achouh PE, Simonet S, Fabiani JN, Verbeuren TJ. Carbon monoxide induces relaxation of human internal thoracic and radial arterial grafts. Interact Cardiovasc Thorac Surg 7: 959 -962, 2008.

2. Agren P, Cogolludo AL, Kessels CG, Perez-Vizcaino F, De Mey JG, Blanco CE, Villamor E. Ontogeny of chicken ductus arteriosus response to oxygen and vasoconstrictors. Am J Physiol Regul Integr Comp Physiol 292: R485-R496, 2007.

3. Agren P, van der Sterren S, Cogolludo AL, Blanco CE, Villamor E. Developmental changes in the effects of prostaglandin E2 in the chicken ductus arteriosus. J Comp Physiol B 179: 133-143, 2009.

4. Agren P, van der Sterren S, Cogolludo AL, Frazziano G, de Mey JG, Blanco CE, Villamor E. Developmental changes in endothelium-depen- dent relaxation of the chicken ductus arteriosus. J Physiol Pharmacol 59: 55-76, 2008.

5. Al-Magableh MR, Hart JL. Mechanism of vasorelaxation and role of endogenous hydrogen sulfide production in mouse aorta. Naunyn Schmie- debergs Arch Pharmacol 383: 403-413, 2011.

6. Altimiras J, Crossley DA 2nd. Control of blood pressure mediated by baroreflex changes of heart rate in the chicken embryo (Gallus gallus). Am J Physiol Regul Integr Comp Physiol 278: R980 -R986, 2000.

7 . Baragatti B, Brizzi F, Barogi S, Laubach VE, Sodini D, Shesely EG, Regan RF, Coceani F. Interactions between NO, $\mathrm{CO}$ and an endothelium-derived hyperpolarizing factor (EDHF) in maintaining patency of the ductus arteriosus in the mouse. $\mathrm{Br} J$ Pharmacol 151: $54-62,2007$.

8. Barbe C, Dubuis E, Rochetaing A, Kreher P, Bonnet P, Vandier C. A 4-AP-sensitive current is enhanced by chronic carbon monoxide exposure in coronary artery myocytes. Am J Physiol Heart Circ Physiol 282: H2031-H2038, 2002.

9. Belanger C, Copeland J, Muirhead D, Heinz D, Dzialowski EM. Morphological changes in the chicken ductus arteriosi during closure at hatching. Anat Rec (Hoboken) 291: 1007-1015, 2008.

10. Bergwerff M, DeRuiter MC, Gittenberger-de Groot AC. Comparative anatomy and ontogeny of the ductus arteriosus, a vascular outsider. Anat Embryol (Berl) 200: 559 $571,1999$.

11. Bilban M, Haschemi A, Wegiel B, Chin BY, Wagner O, Otterbein LE. Heme oxygenase and carbon monoxide initiate homeostatic signaling. J Mol Med 86: 267-279, 2008.

12. Bucci M, Papapetropoulos A, Vellecco V, Zhou Z, Pyriochou A, Roussos C, Roviezzo F, Brancaleone V, Cirino G. Hydrogen sulfide is an endogenous inhibitor of phosphodiesterase activity. Arterioscler Thromb Vasc Biol 30: 1998 -2004, 2010.

13. Buyuknacar HS, Gocmen C, de Groat WC, Kumcu EK, Wu HY, Onder S. Differential effect of L-cysteine in isolated whole-bladder preparations from neonatal and adult rats. J Pharmacol Exp Ther 333: 228 -235, 2010. 
14. Cheang WS, Wong WT, Shen B, Lau CW, Tian XY, Tsang SY, Yao X, Chen ZY, Huang $Y$. 4-Aminopyridine-sensitive $\mathrm{K}+$ channels contributes to NaHS-induced membrane hyperpolarization and relaxation in the rat coronary artery. Vascul Pharmacol 53: $94-982010$.

15. Cheng Y, Ndisang JF, Tang G, Cao K, Wang R. Hydrogen sulfide- induced relaxation of resistance mesenteric artery beds of rats. Am J Physiol Heart Circ Physiol 287: H2316 $-\mathrm{H} 2323,2004$.

16. Coceani F. Carbon monoxide in vasoregulation: the promise and the challenge. Circ Res 86: $1184-1186,2000$.

17. Coceani F, Hamilton NC, Labuc J, Olley PM. Cytochrome P450-linked monooxygenase: involvement in the lamb ductus arteriosus. Am J Physiol Heart Circ Physiol 246: H640-H643, 1984.

18. Coceani F, Kelsey L, Seidlitz E. Carbon monoxide-induced relaxation of the ductus arteriosus in the lamb: evidence against the prime role of guanylyl cyclase. $\mathrm{Br} J$ Pharmacol 118: $1689-1696,1996$.

19. Coceani F, Kelsey L, Seidlitz E, Marks GS, McLaughlin BE, Vreman HJ, Stevenson DK, Rabinovitch M, Ackerley C. Carbon monoxide formation in the ductus arteriosus in the lamb: implications for the regulation of muscle tone. Br J Pharmacol 120: $599-608$, 1997.

20. Cogolludo AL, Moral-Sanz J, van der Sterren S, Frazziano G, van Cleef AN, Menendez C, Zoer B, Moreno E, Roman A, Perez-Vizcaino F, Villamor E. Maturation of $\mathrm{O} 2$ sensing and signaling in the chicken ductus arteriosus. Am J Physiol Lung Cell Mol Physiol 297: L619 -L630, 2009.

21. Conigrave AD, Hampson DR. Broad-spectrum L-amino acid sensing by class 3 Gprotein-coupled receptors. Trends Endocrinol Metab 17: 398 - 407, 2006.

22. d'Emmanuele di Villa Bianca R, Sorrentino R, Maffia P, Mirone V, Imbimbo C, Fusco F, De Palma R, Ignarro LJ, Cirino G. Hydrogen sulfide as a mediator of human corpus cavernosum smooth-muscle relaxation. Proc Natl Acad Sci USA 106: 4513-4518, 2009.

23. Dhaese I, Lefebvre RA. Myosin light chain phosphatase activation is involved in the hydrogen sulfide-induced relaxation in mouse gastric fundus. Eur J Pharmacol 606: 180 $-186,2009$.

24. Dhaese I, Van Colen I, Lefebvre RA. Mechanisms of action of hydrogen sulfide in relaxation of mouse distal colonic smooth muscle. Eur J Pharmacol 628: $179-186$, 2010.

25. di Villa Bianca R, Coletta C, Mitidieri E, De Dominicis G, Rossi A, Sautebin L, Cirino G, Bucci M, Sorrentino R. Hydrogen sulphide induces mouse paw oedema through activation of phospholipase A2. Br J Pharmacol 161: 1835-1842, 2010.

26. Doeller JE, Isbell TS, Benavides G, Koenitzer J, Patel H, Patel RP, Lancaster JR Jr, Darley-Usmar VM, Kraus DW. Polarographic measurement of hydrogen sulfide production and consumption by mammalian tissues. Anal Biochem 341: $40-51,2005$.

27. Dombkowski RA, Doellman MM, Head SK, Olson KR. Hydrogen sulfide mediates hypoxia-induced relaxation of trout urinary bladder smooth muscle. J Exp Biol 209: 3234 
$-3240,2006$.

28. Dombkowski RA, Russell MJ, Olson KR. Hydrogen sulfide as an endogenous regulator of vascular smooth muscle tone in trout. Am J Physiol Regul Integr Comp Physiol 286: R678-R685, 2004.

29. Dombkowski RA, Russell MJ, Schulman AA, Doellman MM, Olson KR. Vertebrate phylogeny of hydrogen sulfide vasoactivity. Am J Physiol Regul Integr Comp Physiol 288: R243-R252, 2005.

30. Dombkowski RA, Whitfield NL, Motterlini R, Gao Y, Olson KR. Effects of carbon monoxide on trout and lamprey vessels. Am J Physiol Regul Integr Comp Physiol 296: R141-R149, 2009.

31. Ewing JF, Raju VS, Maines MD. Induction of heart heme oxygenase-1 (HSP32) by hyperthermia: possible role in stress-mediated elevation of cyclic $3=: 5=-$ guanosine monophosphate. J Pharmacol Exp Ther 271: 408 -414, 1994.

32. Fay FS. Guinea pig ductus arteriosus. I. Cellular and metabolic basis for oxygen sensitivity. Am J Physiol 221: 470 -479, 1971.

33. Flinsenberg TW, van der Sterren S, van Cleef AN, Schuurman MJ, Agren P, Villamor E. Effects of sex and estrogen on chicken ductus arteriosus reactivity. Am J Physiol Regul Integr Comp Physiol 298: R1217-R1224, 2010.

34. Greyner H, Dzialowski EM. Mechanisms mediating the oxygen-induced vasoreactivity of the ductus arteriosus in the chicken embryo. Am J Physiol Regul Integr Comp Physiol 295: R1647-R1659, 2008.

35. Hosoki R, Matsuki N, Kimura $\mathrm{H}$. The possible role of hydrogen sulfide as an endogenous smooth muscle relaxant in synergy with nitric oxide. Biochem Biophys Res Commun 237: 527-531, 1997.

36. Kubo S, Doe I, Kurokawa Y, Kawabata A. Hydrogen sulfide causes relaxation in mouse bronchial smooth muscle. J Pharm Sci 104: 392-396, 2007.

37. Kubo S, Doe I, Kurokawa Y, Nishikawa H, Kawabata A. Direct inhibition of endothelial nitric oxide synthase by hydrogen sulfide: con- tribution to dual modulation of vascular tension. Toxicology 232: 138 - 146, 2007.

38. Lee SW, Cheng Y, Moore PK, Bian JS. Hydrogen sulphide regulates intracellular pH in vascular smooth muscle cells. Biochem Biophys Res Commun 358: 1142-1147, 2007. 39. Leffler CW, Parfenova H, Jaggar JH, Wang R. Carbon monoxide and hydrogen sulfide: gaseous messengers in cerebrovascular circulation. J Appl Physiol 100: 10651076, 2006.

40. Leo MD, Siddegowda YK, Kumar D, Tandan SK, Sastry KV, Prakash VR, Mishra SK. Role of nitric oxide and carbon monoxide in Nm-nitro-L- arginine methyl ester-resistant acetylcholine-induced relaxation in chicken carotid artery. Eur J Pharmacol 596: 111117, 2008.

41. Lim JJ, Liu YH, Khin ES, Bian JS. Vasoconstrictive effect of hydrogen sulfide involves downregulation of cAMP in vascular smooth muscle cells. Am J Physiol Cell Physiol 295: C1261-C1270, 2008.

42. Linden DR, Levitt MD, Farrugia G, Szurszewski JH. Endogenous production of H2S 
in the gastrointestinal tract: still in search of a physiologic function. Antioxid Redox Signal 12: 1135-1146, 2010.

43. Lindgren I, Zoer B, Altimiras J, Villamor E. Reactivity of chicken chorioallantoic arteries, avian homologue of human fetoplacental arteries. J Physiol Pharmacol 61: 619 $-628,2010$.

44. Liu X, Ambudkar IS. Characteristics of a store-operated calcium-perme- able channel: sarcoendoplasmic reticulum calcium pump function controls channel gating. $J$ Biol Chem 276: 29891-29898, 2001.

45. Lowicka E, Beltowski J. Hydrogen sulfide (H2S)-the third gas of interest for pharmacologists. Pharmacol Rep 59: 4 -24, 2007.

46. Moonen RM, Agren P, Cogolludo AL, Vizcaino FP, Villamor E. Response of chicken ductus arteriosus to hypercarbic and normocarbic acidosis. Neonatology 98: 47-56, 2010.

47. Mustafa AK, Gadalla MM, Sen N, Kim S, Mu W, Gazi SK, Barrow RK, Yang G, Wang R, Snyder SH. H2S signals through protein S- sulfhydration. Sci Signal 2: ra72, 2009.

48. Muzaffar S, Shukla N, Bond M, Newby AC, Angelini GD, Sparatore A, Del Soldato P, Jeremy JY. Exogenous hydrogen sulfide inhibits superoxide formation, NOX-1 expression and Rac1 activity in human vascular smooth muscle cells. J Vasc Res 45: 521-528, 2008.

49. Olson KR. Hydrogen sulfide and oxygen sensing: implications in cardiorespiratory control. J Exp Biol 211: 2727-2734, 2008.

50. Olson KR. Is hydrogen sulfide a circulating "gasotransmitter" in vertebrate blood? Biochim Biophys Acta 1787: 856 -863, 2009.

51. Olson KR. The therapeutic potential of hydrogen sulfide: separating hype from hope. Am J Physiol Regul Integr Comp Physiol 301: R297-R312, 2011.

52. Olson KR, Dombkowski RA, Russell MJ, Doellman MM, Head SK, Whitfield NL, Madden JA. Hydrogen sulfide as an oxygen sensor/ transducer in vertebrate hypoxic vasoconstriction and hypoxic vasodilation. J Exp Biol 209: 4011-4023, 2006.

53. Ruijtenbeek K, Kessels CG, Villamor E, Blanco CE, De Mey JG. Direct effects of acute hypoxia on the reactivity of peripheral arteries of the chicken embryo. Am $\mathrm{J}$ Physiol Regul Integr Comp Physiol 283: R331-R338, 2002.

54. Salama G, Abramson JJ, Pike GK. Sulphydryl reagents trigger $\mathrm{Ca2}+$ release from the sarcoplasmic reticulum of skinned rabbit psoas fibres. J Physiol 454: $389-420$, 1992.

55. Shan Y, Lambrecht RW, Bonkovsky HL. Identification of key elements that are responsible for heme-mediated induction of the avian heme oxygenase-1 gene. Biochim Biophys Acta 1679: 87-94, 2004.

56. Sutendra G, Michelakis ED. The chicken embryo as a model for ductus arteriosus developmental biology: cracking into new territory. Am J Physiol Regul Integr Comp Physiol 292: R481-R484, 2007.

57. Szabo C. Hydrogen sulphide and its therapeutic potential. Nat Rev Drug Discov 6: 917-935, 2007. 
58. Tanaka Y, Tang G, Takizawa K, Otsuka K, Eghbali M, Song M, Nishimaru K, Shigenobu K, Koike K, Stefani E, Toro L. Kv channels contribute to nitric oxide- and atrial natriuretic peptide-induced relaxation of a rat conduit artery. J Pharmacol Exp Ther 317: 341-354, 2006.

59. Tang G, Wu L, Liang W, Wang R. Direct stimulation of KATP channels by exogenous and endogenous hydrogen sulfide in vascular smooth muscle cells. Mol Pharmacol 68: 1757-1764, 2005.

60. Teague B, Asiedu S, Moore PK. The smooth muscle relaxant effect of hydrogen sulphide in vitro: evidence for a physiological role to control intestinal contractility. $\mathrm{Br} \mathrm{J}$ Pharmacol 137: 139-145, 2002.

61. Van der Sterren S, Agren P, Zoer B, Kessels L, Blanco CE, Villamor E. Morphological and functional alterations of the ductus arteriosus in a chicken model of hypoxia-induced fetal growth retardation. Pediatr Res 65: 279 -284, 2009.

62. Villamor E, Perez-Vizcaino F, Cogolludo AL, Conde-Oviedo J, Zara- goza-Arnaez F, Lopez-Lopez JG, Tamargo J. Relaxant effects of carbon monoxide compared with nitric oxide in pulmonary and systemic vessels of newborn piglets. Pediatr Res 48: 546 -553, 2000.

63. Villamor E, Ruijtenbeek K, Pulgar V, De Mey JG, Blanco CE. Vascular reactivity in intrapulmonary arteries of chicken embryos during transition to ex ovo life. Am J Physiol Regul Integr Comp Physiol 282: R917-R927, 2002.

64. Wang R. Two's company, three's a crowd: can H2S be the third endogenous gaseous transmitter? FASEB J 16: 1792-1798, 2002.

65. Webb GD, Lim LH, Oh VM, Yeo SB, Cheong YP, Ali MY, El Oakley R, Lee CN, Wong PS, Caleb MG, Salto-Tellez M, Bhatia M, Chan ES, Taylor EA, Moore PK. Contractile and vasorelaxant effects of hydrogen sulfide and its biosynthesis in the human internal mammary artery. J Pharmacol Exp Ther 324: 876 -882, 2008.

66. Whiteman M, Li L, Kostetski I, Chu SH, Siau JL, Bhatia M, Moore PK. Evidence for the formation of a novel nitrosothiol from the gaseous mediators nitric oxide and hydrogen sulphide. Biochem Biophys Res Commun 343: 303-310, 2006.

67. Wu L, Wang R. Carbon monoxide: endogenous production, physiological functions, and pharmacological applications. Pharmacol Rev 57: 585-630, 2005.

68. Xi Q, Tcheranova D, Parfenova H, Horowitz B, Leffler CW, Jaggar JH. Carbon monoxide activates $\mathrm{KCa}$ channels in newborn arteriole smooth muscle cells by increasing apparent $\mathrm{Ca} 2+$ sensitivity of alpha- subunits. Am J Physiol Heart Circ Physiol 286: $\mathrm{H} 610-\mathrm{H} 618,2004$.

69. Zhao W, Wang R. H2S-induced vasorelaxation and underlying cellular and molecular mechanisms. Am J Physiol Heart Circ Physiol 283: H474 - H480, 2002.

70. Zhao W, Zhang J, Lu Y, Wang R. The vasorelaxant effect of $\mathrm{H} 2 \mathrm{~S}$ as a novel endogenous gaseous KATP channel opener. EMBO J 20: 6008 -6016, 2001.

71. Zhao YJ, Wang J, Rubin LJ, Yuan XJ. Inhibition of $\mathrm{Kv}$ and $\mathrm{KCa}$ channels antagonizes NO-induced relaxation in pulmonary artery. Am J Physiol Heart Circ Physiol 272: H904-H912, 1997. 
72. Zoer B, Cogolludo AL, Perez-Vizcaino F, De Mey JG, Blanco CE, Villamor E. Hypoxia sensing in the fetal chicken femoral artery is mediated by the mitochondrial electron transport chain. Am J Physiol Regul Integr Comp Physiol 298: R1026 -R1034, 2010.

73. Zoer B, Kessels L, Vereijken A, De Mey JG, Bruggeman V, Decuypere E, Blanco CE, Villamor $\mathrm{E}$. Effects of prenatal hypoxia on pulmonary vascular reactivity in chickens prone to pulmonary hypertension. J Physiol Pharmacol 60: 119 -130, 2009. 
Chapter 6

\section{Contractile effects of 15-E2t-isoprostane and 15-F2t-isoprostane on chicken embryo ductus arteriosus}

Saskia van der Sterren, Eduardo Villamor

Comparative Biochemistry and Physiology,

Part A 159 (2011) 436-444 


\begin{abstract}
Isoprostanes (IsoPs) are prostaglandin (PG)-like compounds produced nonenzymatically by free radical-catalyzed peroxidation of arachidonate. Cyclooxygenase-derived PGs play a major role in ductus arteriosus (DA) homeostasis but the putative role of IsoPs has not been studied so far. We investigated, using wire myography, the vasoactive effects of $15-\mathrm{E}_{2 \mathrm{t}}-\mathrm{IsoP}$ and $15-\mathrm{F}_{2 \mathrm{t}}$-IsoP in the chicken embryo DA, pulmonary artery (PA) and femoral artery (FA). 15- $E_{2 t}$ - IsoP and 15- $F_{2 t}$-IsoP contracted DA, PA, and FA rings in a concentration-dependent manner. $15-E_{2 t}$-IsoP was equally efficacious (mean \pm SE $E_{\max }=1.25 \pm 0.06 \mathrm{mN} / \mathrm{mm}$ ) as and more potent (-log of molar concentration producing $50 \%$ of $E_{\max }=p E_{50}=7.00 \pm 0.04$ ) than the thromboxane-prostanoid (TP) receptor agonist U46619 $\left(E_{\max }=1.49 \pm 0.11 \mathrm{mN} / \mathrm{mm} ; \mathrm{pEC}_{50}=6.48 \pm 0.05\right)$ in contracting chicken DA (pulmonary side). $15-\mathrm{F}_{2 t}$-IsoP was less potent ( $\mathrm{pE} \mathrm{C}_{50}$ $=5.74 \pm 0.11)$ and less efficacious $\left(E_{\max }=0.96 \pm 0.11\right)$ than U46619. Concentration-dependent contractions to $15-E_{2 t}-I s o P$ and $U 46619$ in DA rings were competitively inhibited by the TP receptor antagonist SQ29548 $(0.1 \mu \mathrm{M}$ to $10 \mu \mathrm{M}$ ) with no decrease in the $E_{\max }$ values. SQ29548 also inhibited concentration-dependent contraction to $15-\mathrm{F}_{2 t}$-IsoP but this inhibition was associated with a decrease in $E_{\max }$. Pre-incubation of DA rings with $15-F_{2 t}-I s o P$ inhibited responses to $U 46619$ and, in vessels contracted with $U 46619(1 \mu \mathrm{M})$, $15-\mathrm{F}_{2 \mathrm{t}}$-IsoP $(>1 \mu \mathrm{M})$ evoked a relaxant response. Enzyme immunoassay did not show a measurable release of $15-F_{2 t}$-IsoP by $D A$ rings. In conclusion, $15-E_{2 t}{ }^{-}$ IsoP is a potent and efficacious constrictor of chicken DA, acting through TP receptors. In contrast, $15-\mathrm{F}_{2 t}-\mathrm{IsOP}$ is probably acting as a partial agonist at TP receptors. We speculate that IsoPs play a role in the control of chicken DA tone and could participate in its closure.
\end{abstract}




\subsection{Introduction}

Isoprostanes (IsoPs), like their stereoisomers prostaglandins (PGs), are cyclic metabolites derived in vivo from the oxidation of arachidonate. However, whereas PG formation is controlled by cyclooxygenase (COX) enzymes, the production of IsoPs occurs nonenzymatically by free radical-mediated peroxidation of phospholipid-bound arachidonate [22,31]. Initially, IsoPs were recognized as valuable markers of oxidative stress and numerous pathological conditions have been shown to be associated with increases in urinary, plasma, and tissue levels of IsoPs. Later, it became apparent that IsoPs exhibit significant bioactivity and could contribute to the functional consequences of oxidant injury and mediate many of the features of the disease states for which they are used as indicators [7,23]. The potential (patho)physiological role of IsoPs might be of particular importance during the fetal and neonatal period, given that their contents in body fluids during these stages are many-fold higher than later in life $[7,13,18,39]$.

There is ample evidence that IsoPs exert a wide variety of actions on vascular smooth muscle and endothelial cells. Many have described contractile responses to IsoPs in numerous vascular tissues from several mammalian species, generally via direct or indirect stimulation of thromboxane (TX)prostanoid (TP) receptors $[7,19,23,32,36]$. In addition, and depending on the compound and the vascular bed, vasodilatory actions of IsoPs have been identified $[20,24,49,50]$. These actions were frequently masked by the vasoconstrictive effects of IsoPs on TP receptors and were only observed when these receptors were blocked by antagonists (such as SQ29548 or ICI 192605), or saturated by an agonist (such as U46619) [6,24,49,50].

The ductus arteriosus (DA) is a large fetal shunt connecting the pulmonary artery to the aorta, allowing most of the right ventricular output to bypass the unexpanded lungs [9]. COX- derived prostanoids, and more particularly PGE 2 , play a major role in prenatal patency and postnatal closure of the mammalian DA, namely during fetal life by exerting a potent relaxant effect and after birth by abruptly withdrawing its action [10,40]. Surprisingly, despite the prominent role of COX products in DA homeostasis, the possible ductal actions of COXindependent PG isomers (i.e. IsoPs) have not been investigated so far.

In the last years, the chicken embryo has emerged as a suitable model for the study of DA developmental biology. Our group and another laboratory have characterized the responsiveness of the chicken DA to several mediators which participate in the control of ductal tone in mammals, including $P G s, \mathrm{O}_{2}$, nitric oxide, endothelin-1 and catecholamines $[1,2,3,5,12,17,21,45]$. In the present 
study, we hypothesized that IsoPs are vasoactive in the (chicken) DA. Using wire myography, we analyzed the mechanical responses induced by $15-E_{2 t}-I_{s o P}$ and $15-F_{2 t}$-IsoP in chicken embryo DA rings. We also evaluated the endogenous production of IsoPs by the chicken DA and the response of chicken embryo pulmonary and systemic (femoral) arteries to exogenous IsoPs.

\subsection{Methods}

\subsubsection{Egg incubation and vessel isolation}

All experimental procedures were carried out according to the regulations of the Dutch Law on Animal Experimentation and the European Directive for the Protection of Vertebrate Animals Used for Experimental and Other Scientific Purposes (86/609/EU). Fertilized eggs of White Leghorn chickens (Gallus gallus domesticus) (Het Anker BV, Ochten, The Netherlands) were incubated at a temperature of $37.8{ }^{\circ} \mathrm{C}, 45 \%$ air humidity and automatically rotated once every hour over an angle of $90^{\circ}$ (incubator model 25HS, Masalles Comercial, Spain). Embryos incubated for 15 (15-d) and 19 days (19-d) of the 21-d incubation period were studied. The majority of the experiments were performed in 19-d (non-internally pipped) embryos, while for the study of developmental changes the two ages were compared. On the experimental day, the embryos were taken out, immediately killed by decapitation and a midline laparotomy and sternotomy were performed. With the aid of a dissecting microscope, the right and the left DA were carefully dissected free from surrounding tissue, severed distal to the takeoff of the right or left pulmonary artery and proximal to the insertion into the aorta, and divided in two segments referred to as pulmonary side and aortic side (PulmDA and AoDA, respectively). The boundary between pulmonary and aortic side was determined based on the marked differences in diameter observed along the chicken DA [1]. In some embryos, rings of the femoral and the caudomedial intrapulmonary artery were also obtained, as previously described [46].

\subsubsection{Recording of vascular reactivity}

Two stainless steel wires (diameter $40 \mu \mathrm{m}$ ) were inserted into the lumen of the vessels, which were mounted as ring segments between an isometric force transducer and a displacement device in a myograph (A/S model $610 \mathrm{M}$; Danish Myo Technology, Aarhus, Denmark). The myograph organ bath (5-mL vol.) was filled with Krebs-Ringer bicarbonate (KRB) buffer, maintained at $39{ }^{\circ} \mathrm{C}$. After a 30-min equilibration period, the vessels were distended to a resting tension 
corresponding to a transmural pressure of $10 \mathrm{mmHg}$ (15-d embryos) or 20 $\mathrm{mmHg}$ (19-d embryos). These pressures correspond to the mean arterial blood pressure reported in chicken embryos at the corresponding age (Altimiras and Crossley, 2000) and elicit the highest contractile response to $\mathrm{KCl}$, as determined in previous experiments (Moonen et al. 2010, Zoer et al., 2010). The vessels were allowed a 30 min equilibration period under basal tone, before eliciting a control contraction by raising the $\mathrm{K}^{+}$concentration of the buffer to $62.5 \mathrm{mM}$ in exchange for $\mathrm{Na}^{+}$. During this first phase of mounting and stabilization, rings were maintained in $\mathrm{KRB}$ aerated with $95 \% \mathrm{~N}_{2} / 5 \% \mathrm{CO}_{2}\left(\mathrm{Po}_{2} 2.48 \pm 0.10 \mathrm{kPa}, n=\right.$ 12, measured with an ABL 510 blood gas analyzer, Radiometer Copenhagen, Denmark). Afterwards, the gas mixture was switched to $21 \% \mathrm{O}_{2} / 74 \% \mathrm{~N}_{2} / 5 \%$ $\mathrm{CO}_{2}\left(\mathrm{Po}_{2} 19.16 \pm 0.33 \mathrm{kPa}, n=12\right)$ to reproduce post-hatching oxygenation. Some experiments, however, were performed under $95 \% \quad \mathrm{~N}_{2} / 5 \% \quad \mathrm{CO}_{2}$ (see results). Cumulative concentration $(0.1 \mathrm{nM}-10 \mu \mathrm{M})$-response curves were constructed for $15-E_{2 t}-I s o P$ (also known as 8-iso- $P G_{2}$ ), 15- $F_{2 t}$-IsoP (also known as 8- iso-PGF 2 ) and the TP receptor agonist $U 46619$ in quiescent vessels as well as in vessels contracted with U46619 $(1 \mu \mathrm{M}), \mathrm{KCl}(62.5 \mathrm{mM})$ or norepinephrine $(\mathrm{NE}, 1 \mu \mathrm{M})$ ). In order to assess the contribution of TP receptors to IsoP- and U46619-mediated contractions, some experiments were performed in the presence of the TP receptor antagonist SQ29548 $(0.1 \mu \mathrm{M}-10 \mu \mathrm{M})$.

\subsubsection{Release of IsoPs}

In order to measure the possible release of IsoPs, 19-d PulmDA rings were placed in thermostated mini-chambers containing $0.5 \mathrm{~mL}$ of $\mathrm{KRB}$ buffer $\left(39^{\circ} \mathrm{C}\right)$ aerated with $21 \% \mathrm{O}_{2} / 74 \% \mathrm{~N}_{2} / 5 \% \mathrm{CO}_{2}$, as previously described (Schuurman and Villamor, 2010). The rings were allowed to equilibrate for $60 \mathrm{~min}$ (in the absence or presence of $10 \mu \mathrm{M}$ indomethacin) before exposing them to a single concentration of acetylcholine $(10 \mu \mathrm{M})$ or vehicle for $10 \mathrm{~min}$. The rings were then removed and the mini-chambers were freeze-clamped in liquid nitrogen and stored at $-80{ }^{\circ} \mathrm{C}$ for further analysis. The concentration of $15-\mathrm{F}_{2 \mathrm{t}}$-IsoP (8iso- $\mathrm{PGF}_{2 \mathrm{a}}$ ) was determined in undiluted $50 \mathrm{~mL}$ samples with an enzyme immunoassay kit purchased from Cayman Chemical (Ann Arbor, MI, USA). The assay was performed according to the manufacturer's instructions.

\subsubsection{Drugs and solutions}

KRB contained (in mmol L-1): $\mathrm{NaCl}$ (118.5), $\mathrm{KCl}$ (4.75), $\mathrm{MgSO}_{4}$ (1.2), $\mathrm{KH}_{2} \mathrm{PO}_{4}$ (1.2), $\mathrm{NaHCO}_{3}$ (25.0), $\mathrm{CaCl}_{2}$ (2.5), glucose (5.5). Solutions containing different concentrations of $\mathrm{K}^{+}$were prepared by replacing $\mathrm{NaCl}$ by an equimolar amount of $\mathrm{KCl}$. 15-E2t-IsoP, 15-F2t- IsoP, U46619 (9,11-dideoxy-11a,9a-epoxymethano- 
$P G F_{2 a}$ ) and SQ29548 were obtained from Cayman Chemical. All other drugs were obtained from Sigma-Aldrich Chemical (St. Louis, MO, USA). IsoPs, U46619 and SQ29548 were initially dissolved in dimethylsulphoxide (DMSO). Further dilations were made in distilled deionized water. The final concentration of DMSO in the myograph baths did not exceed $0.1 \%$, which we have found previously to have little or no effect on mechanical activity $[1,45]$.

\subsubsection{Data analysis}

Data are presented as the mean \pm SE for $n$ embryos. Contraction is expressed in terms of active wall tension, calculated as the force divided by twice the length of the arterial segment $(\mathrm{mN} / \mathrm{mm})$. In order to compare DA, FA and PA rings, the contractions depicted in Table 6.1 (fourth column) are also expressed as percentage of the reference contraction to $\mathrm{KCl}(62.5 \mathrm{mM})$, obtained in each individual ring at the beginning of the experiment. The concentration-response curves for all agonists were analysed using non-linear regression to provide estimates of the $\mathrm{EC}_{50}$ (the agonist concentration that produces $50 \%$ of the maximal response). Potency of agonists is expressed as pEC $\mathrm{C}_{50}\left(-\log \mathrm{EC}_{50}\right)$ and efficacy as maximal response $\left(E_{\max }\right)$. For the antagonist assays, agonist concentration-response curves (in the absence and presence of different concentrations of the antagonist) were fitted to the modified Gaddum/Schild model. When criteria for competitive antagonism were satisfied, which means that the antagonist produced a parallel rightward shift of the agonist curve without attenuation in the $E_{\max }$, antagonist $\mathrm{pA}_{2}$ value and slope of Schild's regression were calculated. The $\mathrm{pA}_{2}$ represents the negative logarithm of the concentration of antagonist that induces a 2-fold shift of the concentration response curve to the right. Terms and equations are as recommended by the International Union of Pharmacology (IUPHAR) Committee on Receptor Nomenclature and Drug Classification (Neubig et al., 2003). Differences between mean values were assessed by unpaired $t$-tests or one-way ANOVA, followed by Bonferroni post hoc test. Differences were considered significant at $P<0.05$. All analyses were performed using a commercially available graph and statistics package (GraphPad Prism version 5, GraphPad InStat version 3.00; GraphPad Software Inc, San Diego, CA, USA). 
Table 6.1. Mean \pm SE efficacy $\left(E_{\max }\right)$ and potency $(p E C 50)$ of prostanoids in chicken embryo vessels.

\begin{tabular}{|c|c|c|c|c|c|}
\hline \multirow[t]{2}{*}{ Vessel } & \multirow[t]{2}{*}{ Compound } & \multicolumn{2}{|l|}{$\mathrm{E}_{\max }$} & \multirow[t]{2}{*}{$\mathrm{pEC}_{50}$} & \multirow[t]{2}{*}{$n$} \\
\hline & & $\mathrm{mN} / \mathrm{mm}$ & $\% \mathrm{KCl}$ & & \\
\hline \multirow[t]{3}{*}{ PulmDA 19-d } & $15-\mathrm{E}_{2 \mathrm{t}}$-IsoP & $1.25 \pm 0.06^{\mathrm{a}, \mathrm{b}}$ & $322.70 \pm 34.03$ & $7.00 \pm 0.04^{a}$ & 16 \\
\hline & $15-\mathrm{F}_{2 \mathrm{t}}$-IsoP & $0.96 \pm 0.11^{\mathrm{b}}$ & $207.00 \pm 13.62$ & $5.74 \pm 0.11^{b}$ & 12 \\
\hline & U-46619 & $1.49 \pm 0.11^{\mathrm{a}}$ & $353.15 \pm 24.79$ & $6.48 \pm 0.08^{c}$ & 12 \\
\hline \multirow[t]{3}{*}{ PulmDA 15-d } & $15-\mathrm{E}_{2 \mathrm{t}}$-IsoP & $0.15 \pm 0.02^{\mathrm{a}}$ & $82.33 \pm 7.76^{*}$ & $6.85 \pm 0.10^{\mathrm{a}}$ & 6 \\
\hline & $15-\mathrm{F}_{2 \mathrm{t}}$-IsoP & $0.08 \pm 0.02^{\mathrm{a}}$ & $53.07 \pm 7.40^{*}$ & $6.06 \pm 0.21^{b}$ & 6 \\
\hline & $\mathrm{U}-46619$ & $0.40 \pm 0.08^{b}$ & $173.91 \pm 34.78^{*}$ & $6.21 \pm 0.06^{\mathrm{b}}$ & 6 \\
\hline \multirow[t]{3}{*}{ AoDA 19-d } & $15-\mathrm{E}_{2 \mathrm{t}}$-IsoP & $0.08 \pm 0.03^{\mathrm{a}}$ & $7.86 \pm 3.93^{*}$ & $6.56 \pm 0.03^{a^{*}}$ & 6 \\
\hline & $15-\mathrm{F}_{2 \mathrm{t}}$-IsoP & $0.11 \pm 0.04^{a}$ & $17.24 \pm 7.61^{* \pi}$ & n.c. & 5 \\
\hline & U-46619 & $0.45 \pm 0.07^{b}$ & $90.55 \pm 14.92^{*}$ & $6.18 \pm 0.05^{\mathrm{b}^{*}}$ & 6 \\
\hline \multirow[t]{3}{*}{ FA 19-d } & $15-\mathrm{E}_{2 \mathrm{t}}$-IsoP & $0.99 \pm 0.21^{\mathrm{a}}$ & $57.52 \pm 12.01^{*}$ & $6.79 \pm 0.06^{\mathrm{a}}$ & 6 \\
\hline & $15-F_{2 t}$-IsoP & $2.11 \pm 0.30^{\mathrm{b}}$ & $124.59 \pm 8.07^{* \dagger}$ & $5.88 \pm 0.06^{\mathrm{b}}$ & 6 \\
\hline & U-46619 & $1.84 \pm 0.25^{\mathrm{a}, \mathrm{b}}$ & $108.64 \pm 14.34^{*}$ & $5.94 \pm 0.06^{\mathrm{b}}$ & 6 \\
\hline \multirow[t]{3}{*}{ PA 19-d } & $15-E_{2 t}-$ IsoP & $0.11 \pm 0.04^{\mathrm{a}}$ & $34.26 \pm 5.82^{*}$ & $6.43 \pm 0.16^{\mathrm{a}^{*} \dagger}$ & 6 \\
\hline & $15-\mathrm{F}_{2 \mathrm{t}}$-IsoP & $0.21 \pm 0.06^{\mathrm{b}}$ & $61.25 \pm 9.95 * \pi$ & $5.97 \pm 0.14^{b}$ & 6 \\
\hline & U-46619 & $0.29 \pm 0.09^{b}$ & $84.21 \pm 13.13^{*}$ & $6.23 \pm 0.15^{\mathrm{a}}$ & 6 \\
\hline
\end{tabular}

DA: ductus arteriosus; Pulm: pulmonary side; Ao: aortic side; FA: femoral artery; PA: pulmonary artery. N.c.: not calculated (maximal effect not reached). Experiments were performed at a $\mathrm{Po}_{2}$ of 19 $\mathrm{kPa}$. In order to compare the efficacy of prostanoids among the different vessel types, the contractions depicted in the fourth column $(\% \mathrm{KCl})$ are expressed as percentage of the reference contraction to $\mathrm{KCl}(62.5 \mathrm{mM})$ obtained in each individual ring at the beginning of the experiment. Values without a common letter (within each vessel) are significantly different $(P<0.05$, ANOVA). $P$ $<0.05$ (ANOVA) vs. same compound in 19-d PulmDA; $\dagger P<0.05$ (ANOVA) vs. same compound in PulmDA 15-d; $\mathbb{T} P<0.05$ (ANOVA) vs. same compound in 19-d FA.

\subsection{Results}

PulmDA rings (19-d) incubated under hypoxic $\left(\mathrm{Po}_{2} 2.5 \mathrm{kPa}\right)$ or normoxic $\left(\mathrm{Po}_{2} 19\right.$ $\mathrm{kPa}$ ) conditions were contracted by $15-\mathrm{E}_{2 \mathrm{t}^{-}}$IsoP (Fig. $6.1 \mathrm{~A}$ and $B$ ) and $15-\mathrm{F}_{2 \mathrm{t}^{-}}$ IsoP (Fig. $6.1 C$ and $D$ ) in a concentration-dependent manner (Table 6.1). Normoxic incubation increased the tension of the rings by $0.31 \pm 0.03 \mathrm{mN} / \mathrm{mm}$ $(n=16)$ but did not significantly change the contractile efficacy of the IsoPs (Fig. $6.1 B$ and $D$ ). In contrast, $15-$ F2t-IsoP was more potent under hypoxic $\left(\mathrm{pEC}_{50}\right.$ $=6.26 \pm 0.09)$ than under normoxic conditions $\left(\mathrm{pEC}_{50}=5.89 \pm 0.07 ; P<0.01\right.$ vs. hypoxic, $t$-test). This effect of $\mathrm{O}_{2}$ tension on the contractile potency was not observed for $15-E_{2 t}$ IsoP. Under normoxic conditions, $15-E_{2 t}$ IsoP was more 
potent than and equally efficacious as the TP receptor agonist U46619 in contracting 19-d PulmDA (Table 6.1). 15- Ftt -IsoP was less potent and less efficacious than U46619 (Table 6.1).

A

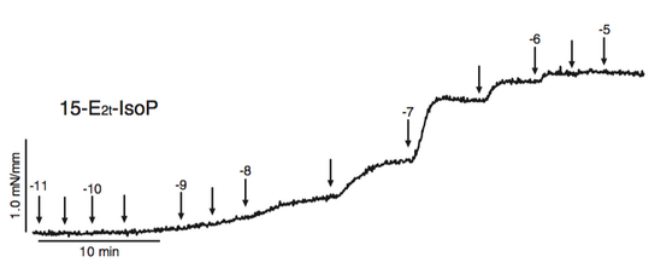

C

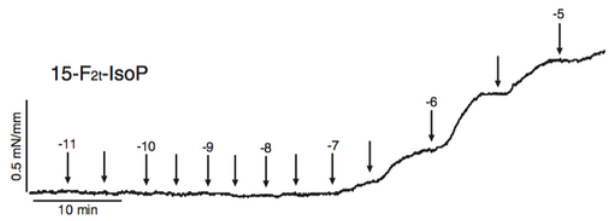

B

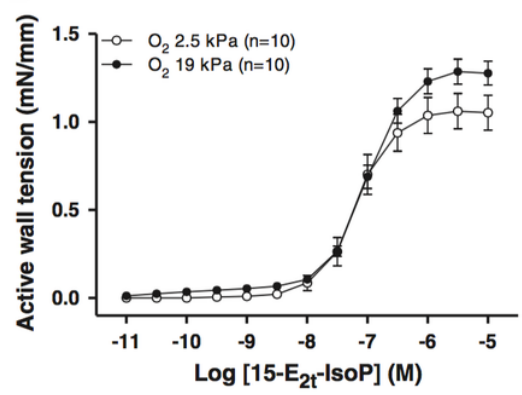

D

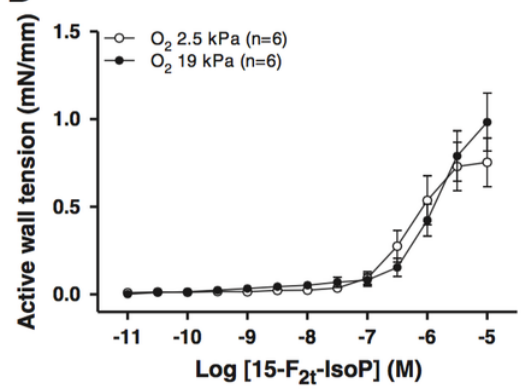

Fig. 6.1. Contractile effects of isoprostanes in chicken embryo DA (19-d, pulmonary side). Representative tracings and mean $( \pm \mathrm{SE})$ data showing the contraction induced by $15-\mathrm{E}_{2 \mathrm{t}}-\mathrm{Iso} \mathrm{P}(A$,

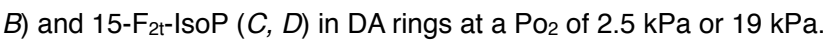

Concentration-response curves to 15-E2t-IsoP (Fig. 6.2A) and U46619 (Fig. $6.2 B$ ) in 19-d PulmDA rings were significantly shifted to the right by increasing concentrations $(0.1 \mu \mathrm{M}-10 \mu \mathrm{M})$ of SQ29548. The $E_{\max }$ was not significantly modified even in the presence of the highest concentration $(10 \mu \mathrm{M})$ of SQ29548, which is indicative of competitive antagonism. The $\mathrm{pA}_{2}$ values for SQ29548 against $15-E_{2 t}$ IsoP and U46619 were $7.29 \pm 0.13$ and $7.13 \pm 0.21$, respectively. The responses to $15-\mathrm{F}_{2 t}$-IsoP in 19 -d PulmDA rings were also inhibited, in a concentration-dependent manner, by SQ29548. However, as can be seen in Fig. 6.2C, the effect of SQ29548 was associated with a decrease in $E_{\max }$ values, suggesting partial agonism of $15-\mathrm{F}_{2 t}-\mathrm{IsoP}$ at TP receptors. In order 
to investigate this, we performed concentration-response curves to U46619 in the absence or presence of $15-\mathrm{F}_{2 t}-\mathrm{IsoP}(10 \mu \mathrm{M})$ and we observed that $15-\mathrm{F}_{2 t^{-}}$ IsoP caused a rightward shift of the U46619 response curve $\left(\mathrm{pEC}_{50}=6.44 \pm\right.$ 0.15 vs. $5.33 \pm 0.13, P<0.01$, $t$-test, Fig. $6.2 D$ ).
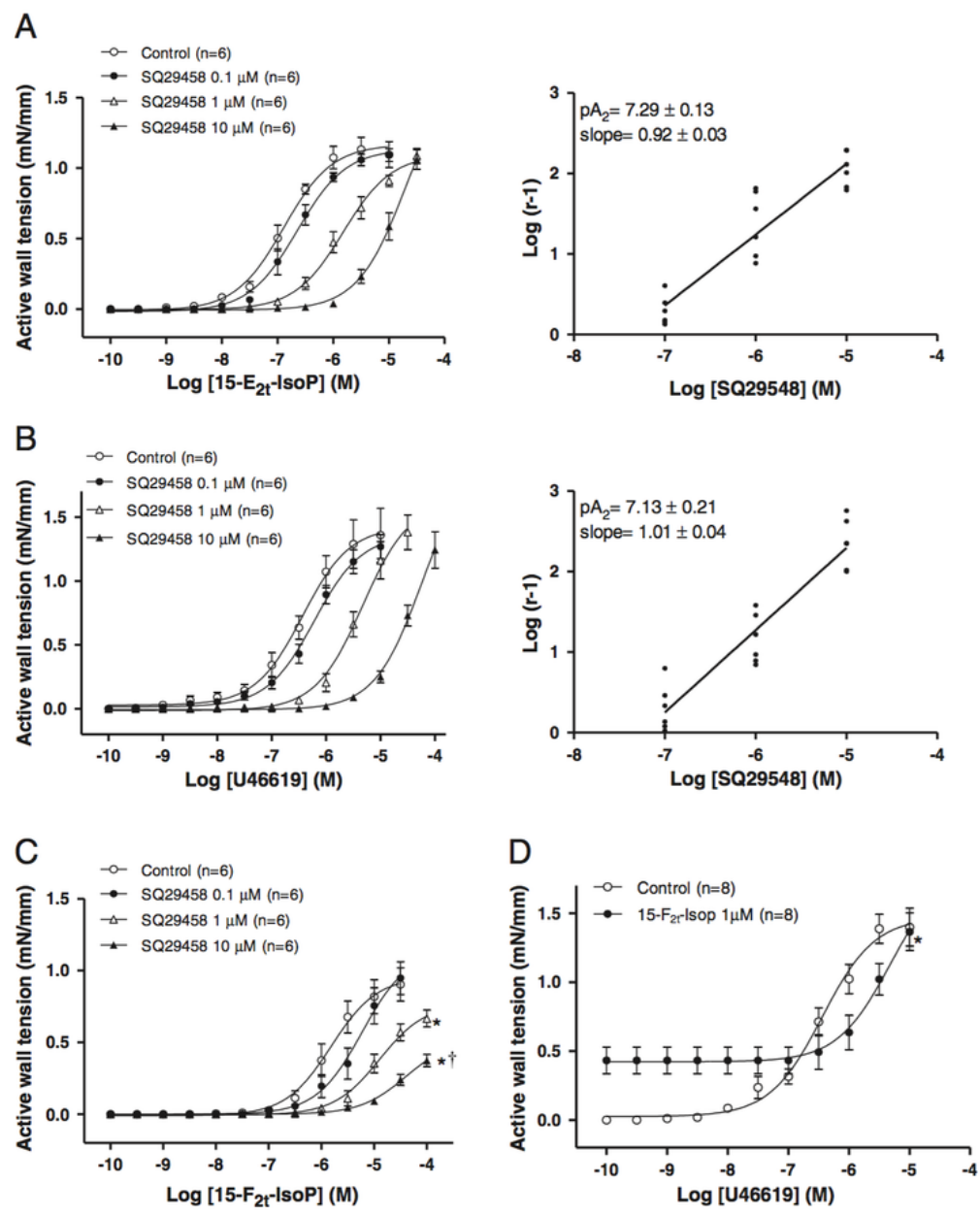

Fig. 6.2. $A-C$ : Mean $( \pm \mathrm{SE})$ concentration-response curves to $15-\mathrm{E}_{2 \mathrm{t}}-\mathrm{IsoP}(A), \mathrm{U} 46619(B)$, and 15$\mathrm{F}_{2 t}$-IsoP $(C)$ in 19-d chicken embryo ductus arteriosus (DA, pulmonary side, $\mathrm{Po}_{2} 19 \mathrm{kPa}$ ) in the absence or the presence of different concentrations of the TP receptor antagonist SQ29548. The right panels in $A$ and $B$ show the Schild plot for SQ29548 vs. 15- $E_{2 t}-I s o P$ and U46619. Schild plot was constructed with concentration-ratios from individual experiments. Due to the high cost of prostanoids, one further concentration was tested only in the $1 \mu \mathrm{M}$ and $10 \mu \mathrm{M}$ SQ29548 groups. $E$ : Mean $( \pm \mathrm{SE})$ concentration-response curves to show the antagonist effect of $15-\mathrm{F}_{2 \mathrm{t}}$-IsoP on U46619-induced contraction in 19-d chicken embryo DA (pulmonary side, $\mathrm{Po}_{2} 19 \mathrm{kPa}$ ). ${ }^{*} P<0.05$ (ANOVA or $t$-test) for difference in $\mathrm{pEC}_{50}$ vs. control. $\dagger P<0.05$ ( $t$-test) for difference in $\mathrm{E}_{\max } \mathrm{vs}$. control. 


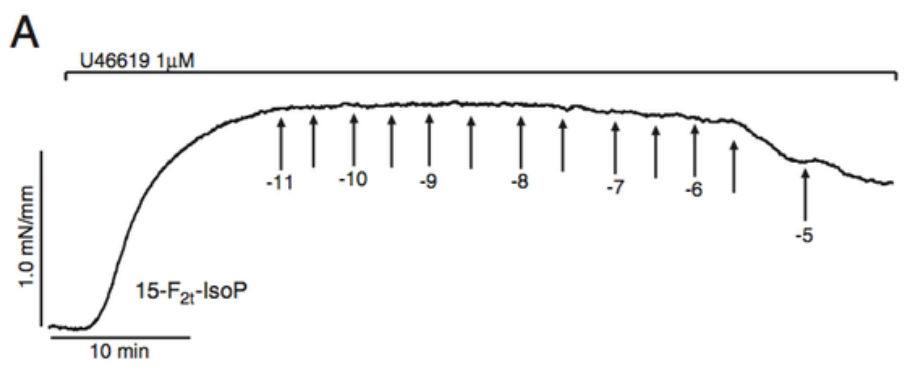

B

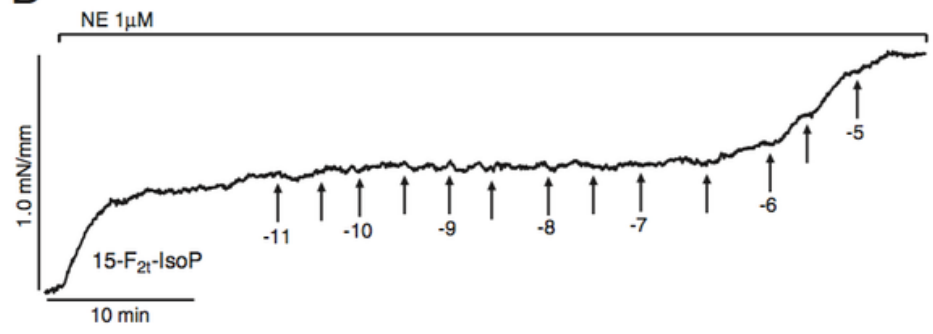

C $\mathrm{KCl} 62.5 \mathrm{mM}+\mathrm{SQ} 2954810 \mu \mathrm{M}$

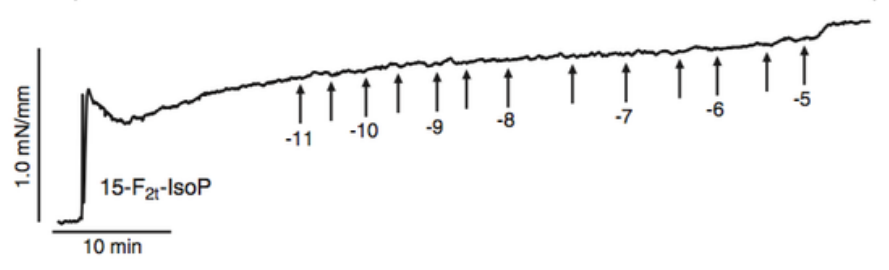

D

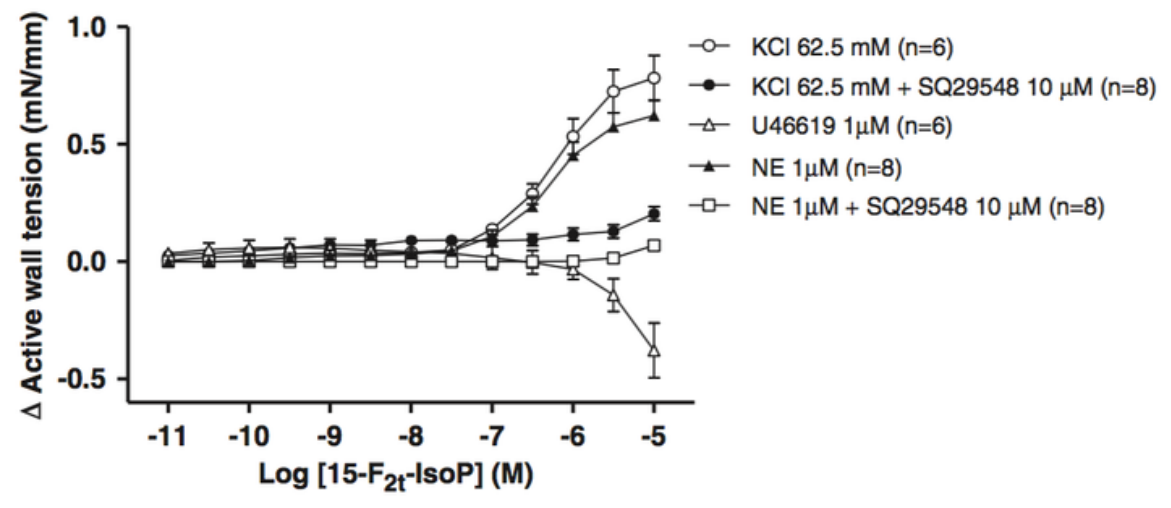

Fig. 6.3. Effects of occupation of TP receptors by the antagonist SQ29548 $(10 \mu \mathrm{M})$ or the agonist U46619 $(1 \mu \mathrm{M})$ on 15- $\mathrm{F}_{2 \mathrm{t}}$-IsoP-mediated contraction of 19-d chicken embryo DA (pulmonary side, $\left.\mathrm{Po}_{2} 19 \mathrm{kPa}\right)$. Representative tracings and mean $( \pm \mathrm{SE})$ data showing the response to $15-\mathrm{F}_{2 t}-\mathrm{IsoP}$ of DA rings contracted with U46619, norepinephrine (NE, in the absence or presence of SQ29548) or $\mathrm{KCl}$ (in the absence or presence of SQ29548). Observe that in the U46619-contracted rings $15-\mathrm{F}_{2 \mathrm{t}}$ IsoP induced a relaxant response. 
In order to investigate the possible relaxant effects of $15-\mathrm{E}_{2 \mathrm{t}}-\mathrm{IsoP}$ and $15-\mathrm{F}_{2 \mathrm{t}^{-}}$ IsoP, PulmDA rings (19-d) were pre-contracted with U46619 $(1 \mu \mathrm{M}), \mathrm{KCl}(62.5$ $\mathrm{mM}$, in the absence or presence of $1 \mu \mathrm{M}$ SQ29548) or NE $(1 \mu \mathrm{M}$, in the absence or presence of $1 \mu \mathrm{M}$ SQ29548). As shown in Fig. 6.3A and $D$, when DA rings were contracted with U46619, 15-F2t-IsoP (at concentrations $>1 \mu \mathrm{M}$ ) induced a relaxation. Also 15-E2t-IsoP (at concentrations $>3 \mu \mathrm{M}$ ) evoked a minimal relaxation (Fig. 6.4A and $C$ ). The relaxant effect of IsoPs was not observed when the vessels were contracted (in the absence or presence of SQ29548) with NE (Fig. 3B and $D$ ) or $\mathrm{KCl}$ (Fig. 6.3C and $D, 6.4 B$ and $C$ ).
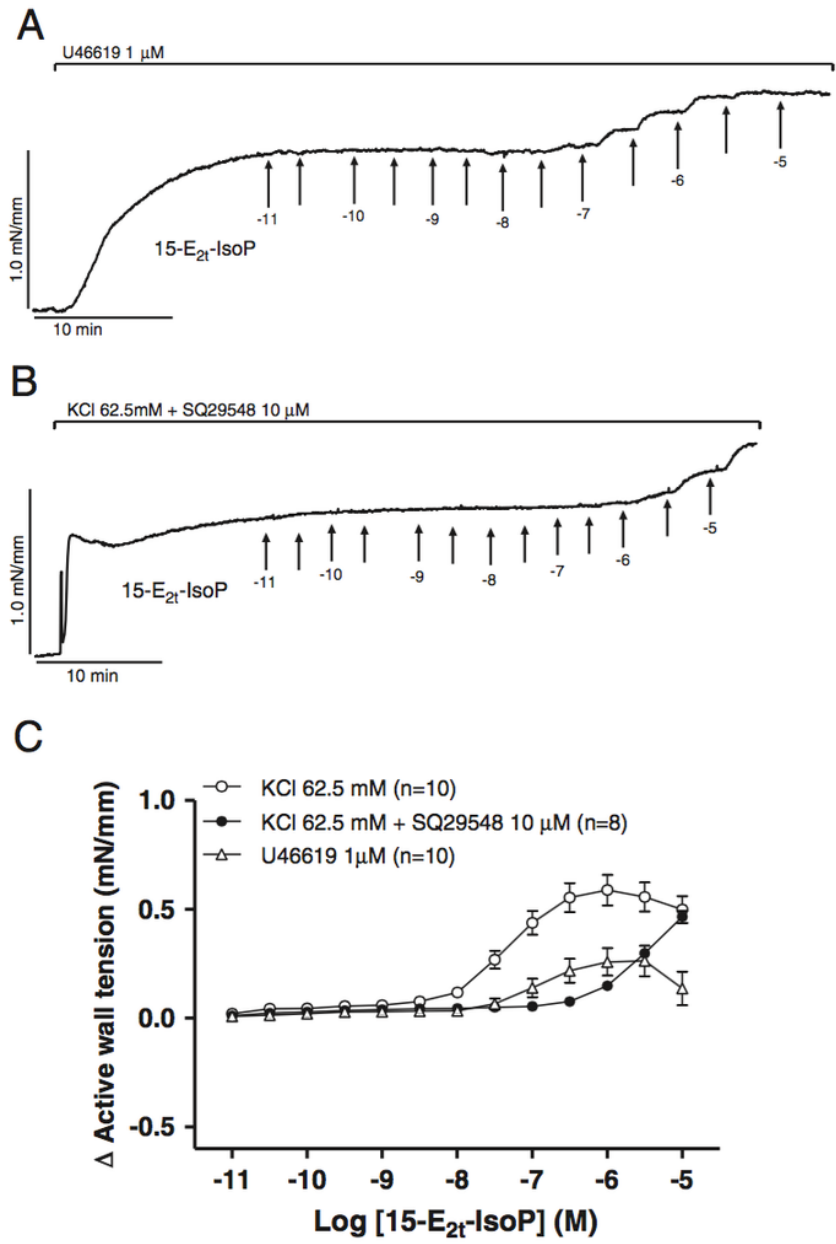

Fig. 6.4. Effects of occupation of TP receptors by the antagonist SQ29548 $(10 \mu \mathrm{M})$ or the agonist U46619 $(1 \mu \mathrm{M})$ on 15- $E_{2 t}$-IsoP-mediated contraction of 19-d chicken embryo DA (pulmonary side, $\left.\mathrm{Po}_{2} 19 \mathrm{kPa}\right)$. Representative tracings and mean $( \pm \mathrm{SE})$ data showing the response to $15-\mathrm{E}_{2 \mathrm{t}}-\mathrm{IsoP}$ of DA rings contracted with $\mathrm{U} 46619$ or $\mathrm{KCl}$ (in the absence or presence of SQ29548). 
Normoxia-incubated 15-d PulmDA rings also showed a concentrationdependent contraction to $15-\mathrm{E}_{2 t} \mathrm{IsoP}, 15-\mathrm{F}_{2 t}$-IsoP and U46619 (Fig. 6.5A-C). These contractions were markedly impaired by the presence of SQ29548 (not shown). $15-E_{2 t}$-IsoP and $15-F_{2 t}$-IsoP were significantly less efficacious than U46619 in contracting 15-d PulmDA rings, but $15-E_{2 t}$ IsoP was more potent than U46619 (Table 6.1). As shown in Fig. 6.5D and 6.7E, when $15 \mathrm{~d}$ PulmDA rings were contracted with U46619, concentrations $>1 \mu \mathrm{M}$ of either $15-E_{2 t}$-IsoP or 15$\mathrm{F}_{2 \mathrm{t}^{-}}$IsoP induced a relaxation. The relaxation induced by $15-\mathrm{E}_{2 \mathrm{t}} \mathrm{IsoP}$ was greater than that induced by $15-\mathrm{F}_{2 t}$-IsoP (Fig. 6.7E).

A

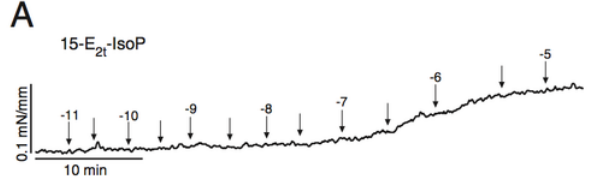

C

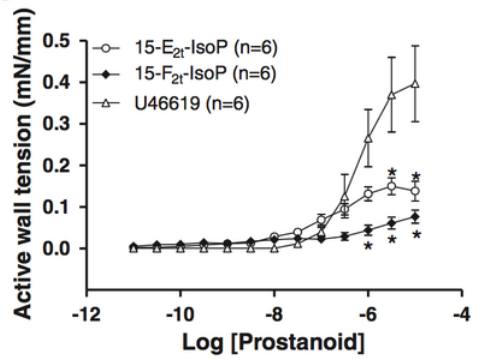

B

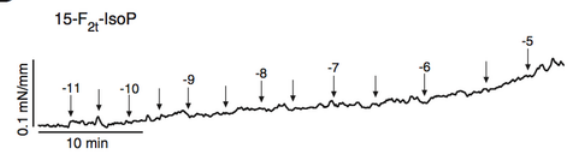

D

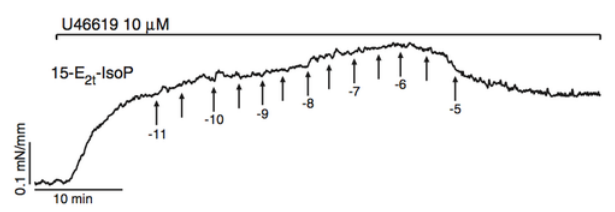

Fig. 6.5. Effects of $15-E_{2 t}-I s o P, 15-F_{2 t}-I s o P$ and $U 46619$ in 15-d chicken embryo ductus arteriosus (DA, pulmonary side; $\left.\mathrm{Po}_{2} 19 \mathrm{kPa}\right)$. Representative tracings and mean $( \pm \mathrm{SE})$ data showing the contraction induced by $15-\mathrm{E}_{2 t}-\mathrm{IsoP}(A, C), 15-\mathrm{F}_{2 t} \mathrm{IsoP}(B, C)$ and $\mathrm{U} 46619(C)$ in quiescent DA rings as well as the relaxation induced by $15-\mathrm{E}_{2 t}-\mathrm{IsoP}(D)$ in rings contracted with U46619 $(1 \mu \mathrm{M})$. ${ }^{*} P<$ 0.05 (ANOVA) vs. U46619.

Normoxia-incubated AoDA rings (19-d) were contracted by $15-\mathrm{E}_{2 t^{-}}$IsoP, $15-\mathrm{F}_{2 t^{-}}$ IsoP, and U46619 (Fig. 6.6) but the three prostanoids showed lower efficacies compared to the ones observed in 19-d PulmDA rings (Table 6.1). 15- $\mathrm{E}_{2 t}-\mathrm{IsoP}$ and $15-F_{2 t}$-IsoP were significantly less efficacious than U46619 in contracting 19-d AoDA rings (Table 6.1). The efficacy of IsoPs to contract AoDA rings was significantly greater when the vessels were incubated under hypoxic conditions (Fig. $6.6 C$ and $D$ ). However, it should be taken into account that normoxic incubation decreased the resting tension of the AoDA rings by $0.28 \pm 0.08 \mathrm{mN} /$ $\mathrm{mm}$. When the AoDA rings were contracted with U46619 (1 $\mu \mathrm{M})$, high 
concentrations $(10 \mu \mathrm{M})$ of $15-\mathrm{E}_{2 \mathrm{t}}$-IsoP and $15-\mathrm{F}_{2 \mathrm{t}}$-IsoP evoked a relaxation (Fig. $6.7 E$ ). This relaxation was not observed when the AoDA rings were contracted with $62.5 \mathrm{mM} \mathrm{KCl}$ (in the absence or presence of SQ29548. Data not shown). Normoxia-incubated 19-d FA (Fig. 6.7A-D and Table 6.1) and PA rings (Table 6.1) showed 15- $E_{2 t}-$ IsoP-, 15- $F_{2 t}$-IsoP- and U46619-induced contractions which were significantly impaired by the presence of SQ29548 (Fig. 6.7C and D. Data not shown for the PA). In the FA and the PA, 15-F2t IsoP and U46619 were more efficacious than 15- $E_{2 t}-$ IsoP (Table 6.1). However, 15- $E_{2 t}$ IsoP was more potent than $15-\mathrm{F}_{2 t}$-IsoP and U46619 in the FA and more potent than $15-\mathrm{F}_{2 t}$-IsoP in the PA (Table 6.1). As shown in Fig. 6.7A and $C$, high concentrations $(\geq 3 \mu \mathrm{M})$ of 15$E_{2 t}$-IsoP relaxed the tone evoked in the FA rings by $1 \mu \mathrm{M} 15-E_{2 t}-$ IsoP. This relaxant effect of $15-E_{2 t}$ IsoP was also observed (Fig. 6.7E) when the FA rings were contracted with $\mathrm{U} 46619(1 \mu \mathrm{M})$ but not when the vessels were contracted with $\mathrm{KCl}(62.5 \mathrm{mM}$ in the absence or presence of SQ29548. Data not shown). $15-\mathrm{F}_{2 t}$ - IsoP did not relax FA rings in any of the experimental conditions (Fig. $6.7 E)$. High concentrations $(10 \mu \mathrm{M})$ of $15-E_{2 t}$ IsoP and $15-F_{2 t}$-IsoP relaxed U46619-contracted PA rings (Fig. 6.7E).

The enzyme immunoassay (limit of detection: $2.7 \mathrm{pg} / \mathrm{mL}$ ) did not show a measurable release of $15-\mathrm{F}_{2 t}$-IsoP (8-iso-PGF $\mathrm{F}_{2 \mathrm{a}}$ ) by $19-\mathrm{d}$ PulmDA rings in any of the above described experimental conditions (see paragraph 6.2 Materials and methods).
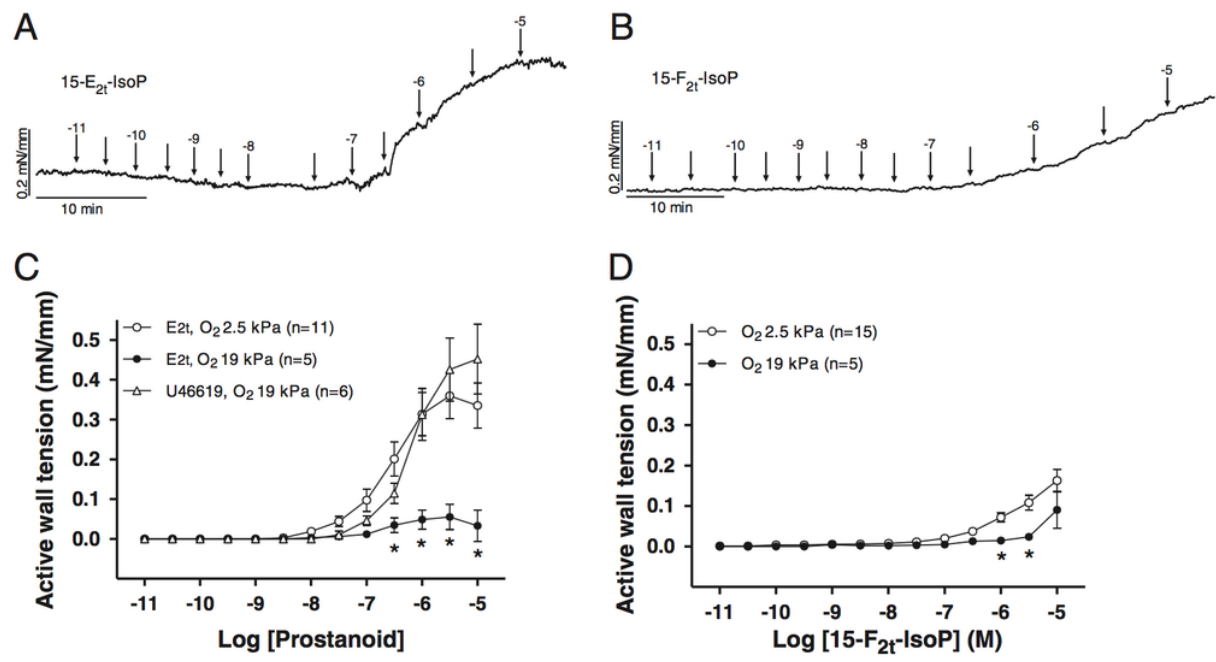

Fig. 6.6. Effects of 15-E2t-IsoP, 15-F2t-IsoP and U46619 in 19-d chicken embryo ductus arteriosus (DA, aortic side). Representative tracings and mean $( \pm S E)$ data showing the contraction induced by 15 -E2t-IsoP (A, C), 15-F2t-IsoP (B, D) and U46619 (C) in quiescent DA rings at a Po2 of $2.5 \mathrm{kPa}$ 
A

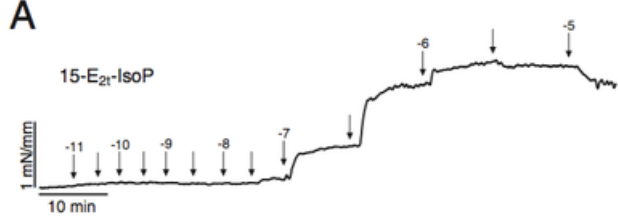

C

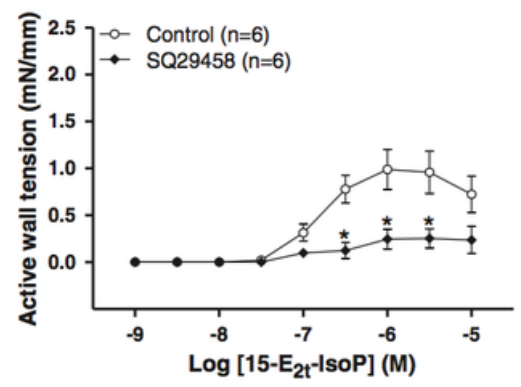

B

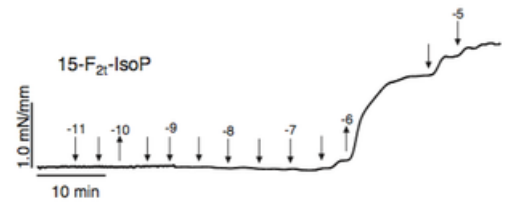

D

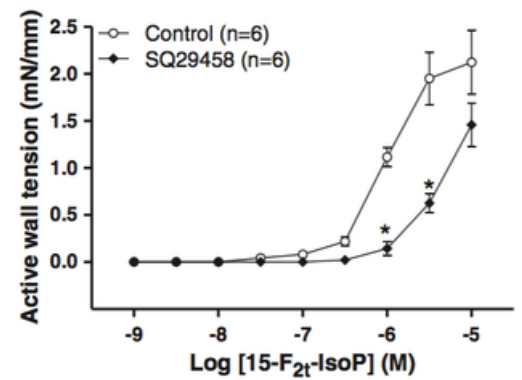

E

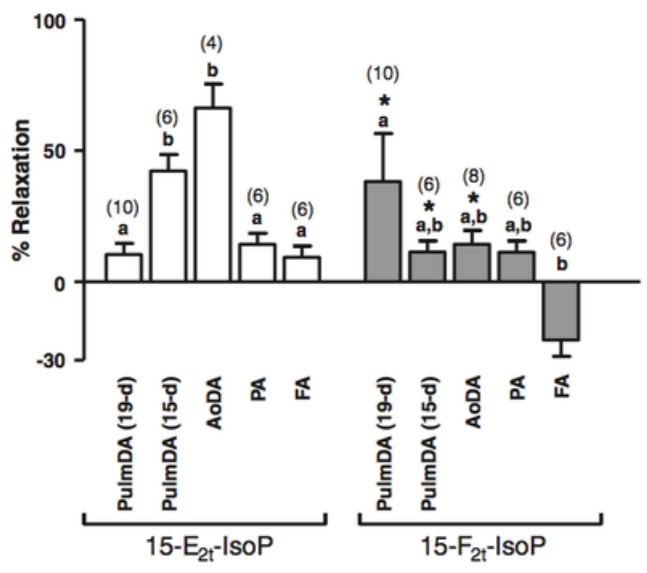

or $19 \mathrm{kPa} .{ }^{*} \mathrm{P}$ b 0.05 (t-test) vs. $2.5 \mathrm{kPa}$.

Fig. 6.7. $A-D$ : Effects of $15-E_{2 t}-I s o P$ and $15-F_{2 t}-I s o P$ in $19-d$ chicken embryo femoral arteries (FA). Representative tracings and mean $( \pm \mathrm{SE})$ data showing the response to $15-\mathrm{E}_{2 t}-\mathrm{IsoP}(A, C)$ and 15$\mathrm{F}_{2 \mathrm{t}}$ IsoP $(B, D)$ in quiescent $\mathrm{FA}$ rings $\left(\mathrm{Po}_{2} 19 \mathrm{kPa}\right)$. The effects of the TP receptor antagonist SQ29548 $(10 \mu \mathrm{M})$ are also shown. ${ }^{*} P<0.05$ (t-test) vs. control. $E$ : Mean $( \pm \mathrm{SE})$ relaxation evoked by $15-E_{2 t}$ IsoP or $15-F_{2 t}-$ IsoP ( $10 \mu \mathrm{M}$ both) in PulmDA (19-d or $\left.15-d\right)$, AoDA, PA, and FA rings contracted with U46619 $(1 \mu \mathrm{M})$. Results are expressed as \% relaxation of U46619-induced tone and negative values indicate contraction. Within each group, columns without a common letter are significantly different $\left(P<0.05\right.$, ANOVA). ${ }^{*} P<0.05$ (t-test) 15 - $E_{2 t}$-IsoP vs. $15-F_{2 t}$-IsoP in the same vessel. Number in parentheses indicates $n$. 


\subsection{Discussion}

In this study, we analyzed for the first time the vasoactive properties of IsoPs in the DA. We observed that $15-E_{2 t}$-IsoP evoked a potent and efficacious, TP receptor-mediated, contraction in the chicken DA. On the other hand, $15-\mathrm{F}_{2 t^{-}}$ IsoP is probably acting as a partial agonist at TP receptors. The contractile response of the PulmDA to IsoPs increased with development and both $15-\mathrm{E}_{2 t^{-}}$ IsoP and $15-\mathrm{F}_{2 t^{-}}$IsoP were particularly efficacious in contracting the PulmDA in comparison with the AoDA and the FA or the PA.

We used the chicken DA as experimental model and therefore its particularities in prostanoid biology need to be considered. In contrast to the DA of the lamb, mouse, rat, rabbit, or pig [40], the chicken DA did not contract in response to COX inhibitors, suggesting that locally derived prostanoids do not exert a tonic vascular effect [1]. However, in a previous study we demonstrated a COXmediated basal production of $\mathrm{PGE}_{2}, \mathrm{PGF}_{2 \mathrm{a}}$ and $\mathrm{TXA}_{2}$ by the chicken $\mathrm{DA}$, which indicates that COX is effectively active in this vessel [38]. The prostanoid receptor gene family lacks a chicken ortholog for the human TP receptor, the Eprostanoid subtype $1\left(E_{1}\right)$ receptor, the I-prostanoid (IP) receptor, and the Dprostanoid (DP) receptor [25]. However, $\mathrm{PGE}_{2}$, the main vasodilator of mammalian DA, elicited in the chicken DA an EP receptor-mediated weak relaxation which was partially or wholly masked by a simultaneous TP receptormediated contraction [3]. Other PGs, such as $\mathrm{PGE}_{1}, \mathrm{PGF}_{2 \mathrm{a}}$, and $\mathrm{PGD}_{2}$, also elicited contraction in the chicken DA $[3,21]$. In addition, high concentrations of the muscarinic agonist acetylcholine induced in the chicken DA an endotheliumdependent contraction, which involved COX-1 activation and TP receptor stimulation. The TP receptor agonist released by acetylcholine was not $\mathrm{PGE}_{2}$, $\mathrm{PGF}_{2 \mathrm{a}}$, or $\mathrm{TXA}_{2}$ [38]. Finally, our present data do not suggest basal or stimulated release of IsoPs by the chicken DA.

Despite the above mentioned lack of avian ortholog of human TP receptor gene, chicken and emu DA as well as chicken embryo pulmonary and systemic arteries are responsive to the TP receptor agonist U46619 $[1,16,26,38,46]$. However, chicken vessels generally show a lower sensitivity to U46619 than mammalian vessels $[11,19,40,42$,$] . On the other hand, the efficacy and the$ potency of U46619 to contract mammalian vessels are generally much higher than the efficacy and potency of IsoPs $[19,24,44]$. Interestingly, this was not the case in chicken embryo vessels and, more particularly, in 19-d PulmDA rings. We observed that $15-E_{2 t}-I s o P$ was more potent than and equally efficacious as U46619 in contracting chicken PulmDA rings. The TP receptor antagonist 
SQ29548 produced a dose-dependent rightward displacement of $15-\mathrm{E}_{2 \mathrm{t}}$-IsoP and U46619 concentration-response curves. This displacement was not accompanied by a decrease in $E_{\max }$ values, fulfilling the criteria of competitive antagonism. Moreover, the $\mathrm{pA}_{2}$ value calculated for SQ29548 vs. $15-\mathrm{E}_{2 \mathrm{t}}$-IsoP was very similar to that calculated for SQ SQ29548 vs.U46619. All of these findings strongly suggest that $15-\mathrm{E}_{2 t}-\mathrm{IsoP}$ mediates contraction through TP receptor stimulation in chicken PulmDA.

The efficacy of $15-\mathrm{F}_{2 t}-\mathrm{IsoP}$ to contract 19-d PulmDA rings was significantly lower than the one observed for U46619. In addition, 15- $\mathrm{F}_{2 t}$-IsoP-induced contraction was inhibited by SQ29548 but the concentration-dependent inhibition was associated with a decrease in the $E_{\max }$ values. This suggests the possibility of a partial agonism of $15-\mathrm{F}_{2 t}-\mathrm{IsoP}$ at TP receptors. The action of a partial agonist requires a higher level of receptor occupancy than that of a full agonist [15]. At high concentrations of SQ29548, the receptor reserve is reduced and $15-\mathrm{F}_{2 t^{-}}$ IsoP is then unable to occupy a sufficient number of receptors to induce a maximal contraction. Added to that, a partial agonist will act as a competitive antagonist of a full agonist acting at the same receptor when present at a concentration that produces a high level of receptor occupancy [35]. Accordingly, we observed an antagonistic effect of $15-\mathrm{F}_{2 t}$-IsoP $(1 \mu \mathrm{M})$ on the contraction induced by U46619 in 19-d PulmDA rings. Moreover, the partial agonism of $15-\mathrm{F}_{2 \mathrm{t}}$-lsoP at the TP receptor may explain the relaxation observed in response to $15-\mathrm{F}_{2 t}$-IsoP on U46619-contracted PulmDA rings. In this case, the level of contraction induced by U46619 $(1 \mu \mathrm{M})$ is close to the maximal contractile response to $15-\mathrm{F}_{2 t}-\mathrm{IsoP}$, leaving no room for an additional contractile effect and therefore unmasking the relaxant effect of the IsoP [15]. Accordingly, the relaxation induced by $15-F_{2 t}-I$ soP was not observed when the DA rings were pre-contracted with an agent different from U46619 (i.e., $\mathrm{KCl}$ or NE) even in the presence of SQ29548. In contrast to the chicken DA, several mammalian vessels when pre-contracted with a non-TP receptor agonist relaxed in response to IsoPs, being generally the E-ring compounds better vasodilators than the F-ring molecules $[7,20,23,24]$.

Interestingly, the high efficacy of IsoPs to contract 19-d PulmDA rings was not observed in the 15-d PulmDA rings. In these vessels, $15-\mathrm{E}_{2 t^{-}}-\mathrm{IsoP}$ and $15-\mathrm{F}_{2 t^{-}}$ IsoP evoked a weak contraction when compared to U46619. Moreover, 15- $E_{2 t}$ IsoP relaxed U46619-contracted 15-d PulmDA rings with higher efficacy than 15- $\mathrm{F}_{2 \mathrm{t}}$-lsoP, suggesting partial TP receptor agonism. 15- $\mathrm{E}_{2 \mathrm{t}}$-IsoP also induced an efficacious relaxation in U46619-contracted AoDA rings. On the basis of our results, we can only speculate on the mechanisms by which $15-E_{2 t}-$ IsoP could act as a partial TP receptor agonist in the 15-d PulmDA and the 19-d AoDA and 
as a full agonist in the 19-d PulmDA. As reviewed by Nakahata [33], TP receptor function is regulated by many factors, such as the glycosylation, the oligomerization, the phosphorylation-dependent desensitization and the internalization of the receptor, as well as the regulation of intracellular trafficking by newly found proteins that interact directly with the receptor. All these factors are susceptible to tissue-dependent and developmental changes.

The chicken DA is the result of the fusion of two vessels with different embryological origin, morphology and functionality. The pulmonary side consists almost exclusively of neural crest derived cells, shows the structure of a muscular artery and responds to $\mathrm{O}_{2}$ with contraction, whereas the aortic part is of mesodermal origin, shows the morphology of an elastic artery and relaxes in response to $\mathrm{O}_{2}[1,5,8,9,12,14,21,45]$. Thus, the PulmDA would be the "real" sixth aortic arch-derived DA, whereas the AoDA is the result of the incorporation of dorsal aorta tissue [9]. The two parts of the DA show marked differences in responsiveness not only to $\mathrm{O}_{2}$ but to several contractile and relaxant agents $[1,2,3,12,30]$. Previously, we reported that the TP receptor-mediated contraction induced by acetylcholine was more marked in the PulmDA [38]. Similarly, IsoPs and the TP receptor agonist U46619 show low efficacy in contracting the AoDA when compared to the PulmDA. This reinforces the idea that TP receptor agonism, either by $\mathrm{TXA}_{2}$ or by IsoPs, might play a key role in chicken DA closure.

As mentioned elsewhere, the putative role of IsoPs in the mammalian DA has not been studied and the majority of the investigations on the effects of prostanoids on DA reactivity focused on the relaxation mediated by COXderived PGs (particularly $\mathrm{PGE}_{2}$ ) through the activation of EP receptors (Smith, 1998; Wright et al., 2001). Nevertheless, the DA of several mammalian species is highly responsive to TP receptor agonists $[37,40,41,42,43]$ and might be also sensitive to IsoPs. Interestingly, Loftin et al. demonstrated that stimulation of TP receptors, but not of EP, IP, or FP receptors, induced DA closure in COXdeficient mice [28]. The deficiency of both COX-1 and COX-2 in mice results in neonatal patent DA and provides a model for identifying the role of prostanoids in ductal closure $[27,28]$. Loftin et al. speculate that the TP receptor-mediated DA contraction in COX-deficient mice may be induced by $\mathrm{PGH}_{2}$, the immediate endoperoxide precursor of PGs and TX [28]. However, IsoPs also appear to be good candidates for this role.

The sharp postnatal transition from the relatively low oxygen intrauterine environment to the significantly higher oxygen extrauterine environment exposes the mammalian newborn to an oxidative challenge. Accordingly, plasma IsoP levels of human newborns are significantly higher than later in life 
and are inversely related to gestational age $[13,18,39]$. The developmental changes observed in the responsiveness to IsoPs in mammalian pulmonary and systemic vessels [7] suggest that these prostanoids may be involved in the control of vascular tone during the perinatal period. Although the increase in arterial oxygenation during avian hatching occurs much slower than during mammalian birth [5], our present data showing IsoP-induced contraction in the chicken DA support the idea of a role for these oxidative stress products on the transitional circulation. However, several questions remain to be elucidated. Some of these questions are: What is the role of IsoPs in the mammalian DA? Is the response to oxidative stress, and consequently the production and role of IsoPs, similar in mammalian and avian species [47]? What is the influence of pre- and postnatal exposure to COX inhibitors on the production and ductal activity of IsoPs [29]? 


\section{References}

1. Agren, P., Cogolludo, A.L., Kessels, C.G., Perez-Vizcaino, F., De Mey, J.G., Blanco, C.E., Villamor, E., 2007. Ontogeny of chicken ductus arteriosus response to oxygen and vasoconstrictors. Am. J. Physiol. Regul. Integr. Comp. Physiol. 292, R485-R496.

2. Agren, P., van der Sterren, S., Cogolludo, A.L., Frazziano, G., de Mey, J.G., Blanco, C.E., Villamor, E., 2008. Developmental changes in endothelium-dependent relaxation of the chicken ductus arteriosus. J. Physiol. Pharmacol. 59, 55-76.

3. Agren, P., van der Sterren, S., Cogolludo, A.L., Blanco, C.E., Villamor, E., 2009. Developmental changes in the effects of prostaglandin E2 in the chicken ductus arteriosus. J Comp Physiol B 179, 133-143.

4. Altimiras, J., Crossley 2nd, D.A., 2000. Control of blood pressure mediated by baroreflex changes of heart rate in the chicken embryo (Gallus gallus). Am. J. Physiol. Regul. Integr. Comp. Physiol. 278, R980-R986.

5. Belanger, C., Copeland, J., Muirhead, D., Heinz, D., Dzialowski, E.M., 2008. Morphological changes in the chicken ductus arteriosi during closure at hatching. Anat Rec Hoboken 291, 1007-1015.

6. Belik, J., Jankov, R.P., Pan, J., Yi, M., Pace-Asciak, C.R., Tanswell, A.K., 2003. Effect of 8-isoprostaglandin F2alpha on the newborn rat pulmonary arterial muscle and endothelium. J. Appl. Physiol. 95, 1979-1985.

7. Belik, J., Gonzalez-Luis, G.E., Perez-Vizcaino, F., Villamor, E., 2010. Isoprostanes in fetal and neonatal health and disease. Free Radic. Biol. Med. 48, 177-188.

8. Bergwerff, M., Verberne, M.E., DeRuiter, M.C., Poelmann, R.E., Gittenberger-de Groot, A.C., 1998. Neural crest cell contribution to the developing circulatory system: implications for vascular morphology? Circ. Res. 82, 221-231.

9. Bergwerff, M., DeRuiter, M.C., Gittenberger-de Groot, A.C., 1999. Comparative anatomy and ontogeny of the ductus arteriosus, a vascular outsider. Anat Embryol Berl 200, 559-571.

10. Clyman, R.I., 2006. Mechanisms regulating the ductus arteriosus. Biol. Neonate 89, 330-335.

11. Cogolludo, A., Frazziano, G., Cobeno, L., Moreno, L., Lodi, F., Villamor, E., Tamargo, J., Perez-Vizcaino, F., 2006. Role of reactive oxygen species in Kv channel inhibition and vasoconstriction induced by TP receptor activation in rat pulmonary arteries. Ann. N.Y. Acad. Sci. 1091, 41-51.

12. Cogolludo, A.L., Moral-Sanz, J., van der Sterren, S., Frazziano, G., van Cleef, A.N., Menendez, C., Zoer, B., Moreno, E., Roman, A., Perez-Vizcaino, F., Villamor, E., 2009. Maturation of $\mathrm{O} 2$ sensing and signaling in the chicken ductus arteriosus. Am. J. Physiol. Lung Cell. Mol. Physiol. 297, L619-L630.

13. Comporti, M., Signorini, C., Leoncini, S., Buonocore, G., Rossi, V., Ciccoli, L., 2004. Plasma F2-isoprostanes are elevated in newborns and inversely correlated to gestational age. Free Radic. Biol. Med. 37, 724-732. 
14. Copeland, J., Dzialowski, E.M., 2009. Effects of hypoxic and hyperoxic incubation on the reactivity of the chicken embryo (Gallus gallus) ductus arteriosi in response to catecholamines and oxygen. Exp. Physiol. 94, 152-161.

15. Cracowski, J.L., Camus, L., Durand, T., Devillier, P., Guy, A., Hardy, G., StankeLabesque, F., Rossi, J.C., Bessard, G., 2002. Response of rat thoracic aorta to F(2)isoprostane metabolites. J. Cardiovasc. Pharmacol. 39, 396-403.

16. Dzialowski, E.M., Greyner, H., 2008. Maturation of the contractile response of the Emu ductus arteriosus. J Comp Physiol B 178, 401-412.

17. Flinsenberg, T.W., van der Sterren, S., van Cleef, A.N., Schuurman, M.J., Agren, P., Villamor, E., 2010. Effects of sex and estrogen on chicken ductus arteriosus reactivity. Am. J. Physiol. Regul. Integr. Comp. Physiol. 298, R1217-R1224.

18. Friel, J.K., Friesen, R.W., Harding, S.V., Roberts, L.J., 2004. Evidence of oxidative stress in full-term healthy infants. Pediatr. Res. 56, 78-882.

19. Gonzalez-Luis, G., Perez-Vizcaino, F., Garcia-Munoz, F., de Mey, J.G., Blanco, C.E., Villamor, E., 2005. Age-related differences in vasoconstrictor responses to isoprostanes in piglet pulmonary and mesenteric vascular smooth muscle. Pediatr. Res. 57, 845-852.

20. Gonzalez-Luis, G., Perez-Vizcaino, F., Blanco, C.E., Villamor, E., 2010. Age-related changes in isoprostane-mediated relaxation of piglet blood vessels. Front Biosci Elite Ed 2, 369-379.

21. Greyner, H., Dzialowski, E.M., 2008. Mechanisms mediating the oxygen-induced vasoreactivity of the ductus arteriosus in the chicken embryo. Am. J. Physiol. Regul. Integr. Comp. Physiol. 295, R1647-R1659.

22. Jahn, U., Galano, J.M., Durand, T., 2008. Beyond prostaglandins-chemistry and biology of cyclic oxygenated metabolites formed by free-radical pathways from polyunsaturated fatty acids. Angew. Chem. Int. Ed. Engl. 47, 5894-5955.

23. Janssen, L.J., Catalli, A., Helli, P., 2005. The pulmonary biology of isoprostanes. Antioxid. Redox Signal. 7, 244-255.

24. Jourdan, K.B., Evans, T.W., Curzen, N.P., Mitchell, J.A., 1997. Evidence for a dilator function of 8-iso prostaglandin F2 alpha in rat pulmonary artery. Br. J. Pharmacol. 120, 1280-1285.

25. Lagerström, M.C., Hellström, A.R., Gloriam, D.E., Larsson, T.P., Schiöth, H.B., Fredriksson, R., 2006. The G protein-coupled receptor subset of the chicken genome. PLoS Comput. Biol. 2, e54.

26. Lindgren, I., Zoer, B., Altimiras, J., Villamor, E., 2010. Reactivity of chicken chorioallantoic arteries, avian homologue of human fetoplacental arteries. J. Physiol. Pharmacol. 61, 619-628.

27. Loftin, C.D., Trivedi, D.B., Tiano, H.F., Clark, J.A., Lee, C.A., Epstein, J.A., Morham, S.G., Breyer, M.D., Nguyen, M., Hawkins, B.M., Goulet, J.L., Smithies, O., Koller, B.H., Langenbach, R., 2001. Failure of ductus arteriosus closure and remodeling in neonatal mice deficient in cyclooxygenase-1 and cyclooxygenase-2. Proc Natl Acad Sci USA 98, 1059-1064.

28. Loftin, C.D., Trivedi, D.B., Langenbach, R., 2002. Cyclooxygenase-1-selective 
inhibition prolongs gestation in mice without adverse effects on the ductus arteriosus. J. Clin. Invest. 110, 549-557.

29. Longini, M., Perrone, S., Vezzosi, P., Proietti, F., Marzocchi, B., Buonocore, G., Fanos, V., Antonucci, R., Brunoldi, E., 2011. Isoprostane levels in urine of preterm newborns treated with ibuprofen for patent ductus arteriosus closure. Pediatr Nephrol 26, 105-109.

30. Moonen, R.M., Agren, P., Cogolludo, A.L., Perez Vizcaino, F., Villamor, E., 2010. Response of chicken ductus arteriosus to hypercarbic and normocarbic acidosis. Neonatology 98, 47-56.

31. Morrow, J.D., Awad, J.A., Boss, H.J., Blair, I.A., Roberts II, L.J., 1992. Noncyclooxygenase- derived prostanoids (F2-isoprostanes) are formed in situ on phospholipids. Proc Natl Acad Sci USA 89, 10721-10725.

32. Mueed, I., Tazzeo, T., Liu, C., Pertens, E., Zhang, Y., Cybulski, I., Semelhago, L., Noora, J., Lamy, A., Teoh, K., Chu, V., Janssen, L.J., 2008. Isoprostanes constrict human radial artery by stimulation of thromboxane receptors, Ca2+ release, and RhoA activation. J Thor Cardiovasc Surg 135, 131-138.

33. Nakahata, N., 2008. Thromboxane A2: physiology/pathophysiology, cellular signal transduction and pharmacology. Pharmacol. Ther. 118, 18-35.

34. Neubig, R.R., Spedding, M., Kenakin, T., Christopoulos, A., Receptor Nomenclature, International Union of Pharmacology Committee on, Drug, Classification, 2003. International Union of Pharmacology Committee on Receptor Nomenclature and Drug Classification. XXXVIII. Update on terms and symbols in quantitative pharmacology. Pharmacol. Rev. 55, 597-606.

35. O'Connor, S.E., Dainty, I.A., Leff, P., 1991. Further subclassification of ATP receptors based on agonist studies. Trends Pharmacol. Sci. 12, 137-141.

36. Oliveira, L., Stallwood, N.A., Crankshaw, D.J., 2000. Effects of some isoprostanes on the human umbilical artery in vitro. Br. J. Pharmacol. 129, 509-514.

37. Reese, J., O'Mara, P.W., Poole, S.D., Brown, N., Tolentino, C., Eckman, D.M., Aschner, J.L., 2009. Regulation of the fetal mouse ductus arteriosus is dependent on interaction of nitric oxide and COX enzymes in the ductal wall. Prostaglandins Other Lipid Mediator 88, 89-96.

38. Schuurman, M.J., Villamor, E., 2010. Endothelium-dependent contraction induced by acetylcholine in the chicken ductus arteriosus involves cyclooxygenase-1 activa- tion and TP receptor stimulation. Comp. Biochem. Physiol. A Mol. Integr. Physiol. 157, 28-34.

39. Signorini, C., Perrone, S., Sgherri, C., Ciccoli, L., Buonocore, G., Leoncini, S., Rossi, V., Vecchio, D., Comporti, M., 2008. Plasma esterified F2-isoprostanes and oxidative stress in newborns: role of nonprotein-bound iron. Pediatr. Res. 63, 287-291.

40. Smith, G.C., 1998. The pharmacology of the ductus arteriosus. Pharmacol. Rev. 50, 35-58.

41. Smith, G.C., McGrath, J.C., 1993. Characterisation of the effect of oxygen tension on response of fetal rabbit ductus arteriosus to vasodilators. Cardiovasc. Res. 27, 22052211. 
42. Smith, G.C., McGrath, J.C., 1995. Contractile effects of prostanoids on fetal rabbit ductus arteriosus. J. Cardiovasc. Pharmacol. 25, 113-118.

43. Trivedi, D.B., Sugimoto, Y., Loftin, C.D., 2006. Attenuated cyclooxygenase-2 expression contributes to patent ductus arteriosus in preterm mice. Pediatr. Res. 60, 669-674.

44. Truog, W.E., Norberg, M., Thibeault, D.W., 1997. Effects of 8-epi-prostaglandin F2 alpha and U46,619 on pulmonary hemodynamics in piglets. Biol. Neonate 71, 306-316.

45. Van der Sterren, S., Agren, P., Zoer, B., Kessels, L., Blanco, C.E., Villamor, E., 2009. Morphological and functional alterations of the ductus arteriosus in a chicken model of hypoxia-induced fetal growth retardation. Pediatr. Res. 65, 279-284.

46. Villamor, E., Ruijtenbeek, K., Pulgar, V., De Mey, J.G., Blanco, C.E., 2002. Vascular reactivity in intrapulmonary arteries of chicken embryos during transition to ex ovo life. Am. J. Physiol. Regul. Integr. Comp. Physiol. 282, R917-R927.

47. West, J.B., 2010. Did differences in mitochondrial properties influence the evolution of avian and mammalian lungs? Am. J. Physiol. Lung Cell. Mol. Physiol. 299, L595-596.

48. Wright, D.H., Abran, D., Bhattacharya, M., Hou, X., Bernier, S.G., Bouayad, A., Fouron, J.C., Vazquez-Tello, A., Beauchamp, M.H., Clyman, R.I., Peri, K., Varma, D.R., Chemtob, S., 2001. Prostanoid receptors: ontogeny and implications in vascular physiology. Am.J. Physiol. Regul. Integr. Comp. Physiol. 281, R1343-R1360.

49. Zhang, Y., Tazzeo, T., Hirota, S., Janssen, L.J., 2003. Vasodilatory and electrophysiological actions of 8-iso-prostaglandin E2 in porcine coronary artery. J. Pharmacol. Exp. Ther. 305, 1054-1060.

50. Zhang, Y., Pertens, E., Janssen, L.J., 2005. 8-isoprostaglandin E(2) activates $\mathrm{Ca}(2+)$ dependent $\mathrm{K}(+)$ current via cyclic AMP signaling pathway in murine renal artery. Eur. $\mathrm{J}$. Pharmacol. 520, 22-28.

51. Zoer, B., Cogolludo, A.L., Perez-Vizcaino, F., De Mey, J.G., Blanco, C.E., Villamor, E., 2010. Hypoxia sensing in the fetal chicken femoral artery is mediated by the mitochondrial electron transport chain. Am. J. Physiol. Regul. Integr. Comp. Physiol. 298, R1026-R1034. 


\section{Chapter 7}

\section{Effects of sex and estrogen on chicken ductus arteriosus reactivity}

Thijs W. H. Flinsenberg, Saskia van der Sterren, Anne N. H. van

Cleef, Marijn J. Schuurman, Pia Ågren, Eduardo Villamor Am J Physiol Regul Integr Comp Physiol 298: R1217-1224, 2010 


\begin{abstract}
Sex hormones have an important influence on cardiovascular physiology and pathophysiology and sex differences in vascular reactivity have been widely demonstrated. In the present study we hypothesized 1) the presence of sexual dimorphism in chicken ductus arteriosus (DA) responsiveness to contractile and relaxant stimuli and 2) that estrogens are vasoactive in the chicken DA. In vitro contractions (assessed with a wire myograph) induced by normoxia, $\mathrm{KCl}, 4-$ aminopyridine, norepinephrine, phenylephrine, U46619, or endothelin-1, as well as relaxations induced by $A C h$, sodium nitroprusside, BAY 41-2272, $P_{G}$, isoproterenol, forskolin, Y-27632, and hydroxyfasudil were not significantly different between males and females. The estrogen $17 \beta$-estradiol elicited concentration-dependent relaxation of $\mathrm{KCl}$, phenylephrine-, and oxygeninduced active tone in male and female chicken DA. The stereoisomer 17aestradiol showed lesser relaxant effects, and the selective estrogen receptor (ER) agonists 4,4',4"-(4-propyl- ['H]pyrazole-1,3,5-triyl)tris-phenol (ERa) and 2,3-bis(4-hydroxy-phenyl)-propionitrile $(E R \beta)$ did not show any effect. There were no sex differences in the responses to estrogen. Endothelium removal or the presence of the soluble guanylate cyclase inhibitor ODQ, the $\mathrm{K}^{+}$channel blockers tetraethylammonium, glibenclamide, and charybdotoxin, or the ER antagonist fulvestrant did not modify $17 \beta$-estradiol-induced relaxation. $\mathrm{CaCl}_{2}(30$ $\mu \mathrm{M}-10 \mathrm{mM}$ ) induced concentration-dependent contraction in DA rings depolarized by $62.5 \mathrm{mM} \mathrm{KCl}$ or stimulated with $21 \% \mathrm{O}_{2}$ in $\mathrm{Ca}^{2+-}$ free medium. Preincubation with $17 \beta$-estradiol or the L-type $\mathrm{Ca}^{2+}$ channel blocker nifedipine produced an inhibition of $\mathrm{CaCl}_{2}$-induced contractions. In conclusion, there are no sex-related differences in chicken DA reactivity. The estrogen $17 \beta$-estradiol induces an endothelium-independent relaxation of chicken DA that is not mediated by ER activation. This relaxant effect is, at least partially, due to inhibition of $\mathrm{Ca}^{2+}$ entry from extracellular space.
\end{abstract}




\subsection{Introduction}

When compared with females, human males are at higher risk of prematurity, as well as pulmonary, neurological, gastrointestinal and cardiovascular prematurityrelated conditions, including failure of closure of the ductus arteriosus (DA) [18, 19 , 50]. This "male disadvantage" with respect to neonatal morbidity and mortality has been recognized for more than three decades [8,34], but the contributing biological mechanisms are poorly understood and likely to be multifactorial. It has been proposed that the male disadvantage begins in utero, when gonadal steroid production already differs strongly by sex [19].

Considerable evidence indicates that sex hormones have an important influence on cardiovascular physiology and pathophysiology, and sex differences in systemic and pulmonary vascular reactivity and pathology have been widely demonstrated $[17,27,35,38,39,44,57,60]$. Estrogen, progesterone, and testosterone receptors are expressed in endothelial and vascular smooth muscle cells, and it is now apparent that these hormones participate in the control of vascular tone [38]. Specifically, free estrogens diffuse through the plasma membrane and form complexes with cytosolic/nuclear receptors, resulting in the induction of genomic effects [7]. Estrogens also bind to plasma membrane receptors in cells and induce rapid nongenomic events $[7,26]$. Estrogen-induced, endothelium-dependent and -independent relaxation has been demonstrated in numerous vascular beds from several species (see Ref. 57 for review). However, neither the presence of sex differences in vascular reactivity nor the vascular effects of sex hormones has been so far investigated in the DA.

In recent years, the chicken embryo has emerged as a suitable model for the study of DA vascular biology [1-4,13,14,21,32,53,54]. Genotypic mechanisms control sex determination in birds like in mammals but, in contrast with mammals, the avian female is heterogametic $(\mathrm{ZW})$ and the male homogametic (ZZ) $[6,9,52]$. In addition, sexual differentiation occurs in opposite manners in birds and mammals. In the latter, the presence of testosterone and other testicular hormones are a preliminary condition for development of male characteristics, and in its absence, development takes the feminine course $[6,9,52]$. In contrast, birds develop a masculine phenotype in the absence of estrogens. In the avian female, the $\mathrm{W}$ chromosome positively controls early aromatase synthesis and consequently estrogen production, which is crucial not only for gonadal sex differentiation but also for subsequent hormonal and phenotypical sexual differentiation [6,9,52]. In the chicken, plasma levels of steroids start to differ between males and females as early as 7.5 days of 
incubation [9]. Therefore, estrogen-dependent sexual differentiation makes the chicken embryo also a suitable organism for testing the potential effects of gonadal steroids during development. In the present study we hypothesized 1) the presence of sexual dimorphism in chicken DA responsiveness to contractile and relaxant stimuli and 2) that estrogens are vasoactive in the chicken DA. To test these hypotheses, we analyzed, using wire myography, the reactivity of DA rings from male and female chicken embryos.

\subsection{Methods}

\subsubsection{Egg incubation and vessel isolation}

All experimental procedures were carried out according to the regulation of the Dutch Law on Animal Experimentation and the European Directive for the Protection of Vertebrate Animals Used for Experimental and Other Scientific Purposes (86/609/EU), and were approved by the Committee on Animal Experimentation of the University of Maastricht. Fertilized eggs of White Leghorn chickens were incubated at $37.8^{\circ} \mathrm{C}, 45 \%$ humidity and rotated once per hour (Incubator model no. 25HS; Masalles Comercial, Barcelona, Spain). Noninternally pipped embryos incubated for 15,17 , or 19 days of the 21-day incubation period were studied. The embryos were taken out of the egg and immediately killed by decapitation; a midline laparotomy and sternotomy were then performed. The sex of the chicken embryos was determined by the in situ microscopic examination of the gonads. In female chickens, only the left gonad develops as an ovary, and on day 15 and onward, the female urogenital already presents a severe dissymmetry (the left ovary is developed and functional, whereas the right gonad is clearly atrophic) [6]. In contrast, the male reproductive tract is a paired system and the two testicles and the two ductus deferens can be clearly visualized by 15 days and onward in chicken embryos [6]. After sex was determined, the right and the left DA were carefully dissected free from surrounding tissue, severed distal to the takeoff of the right or left pulmonary artery and proximal to the insertion into the aorta, and divided into two segments referred to as the pulmonary side and aortic side. The boundary between pulmonary and aortic side was determined on the basis of the marked differences of diameter ob- served along the chicken DA [1].

\subsubsection{Recording of DA reactivity}

Two stainless-steel wires (diameter $40 \mu \mathrm{m}$ ) were inserted into the lumen of the $\mathrm{DA}$, which was mounted as a ring segment between an isometric force 
transducer and a displacement device in a myograph (Danish Myo Technology A/S model 610M, Aarhus, Denmark). The myograph organ bath $(5 \mathrm{ml})$ was filled with Krebs-Ringer bicarbonate buffer (KRB) buffer maintained at $39^{\circ} \mathrm{C}$ and continuously aerated with $95 \% \mathrm{~N}_{2} / 5 \% \mathrm{CO}_{2}\left(\mathrm{Po}_{2} 2.48 \mathrm{kPa}\right.$, SD 0.34, $n=$ 12, measured with an ABL 510 blood gas analyzer, Radiometer Copenhagen, Denmark). Each DA was stretched to its individual optimal lumen diameter, i.e., the diameter at which it developed the strongest contractile response to 62.5 $\mathrm{mM} \mathrm{K}^{+}$, using a diameter-tension protocol as previously described [1,54]. The response of the DA rings to normoxia was assessed by bubbling the organ chamber with $21 \% \mathrm{O}_{2} / 74 \% \mathrm{~N}_{2} / 5 \% \mathrm{CO}_{2}\left(\mathrm{Po}_{2} 19.16 \mathrm{kPa}, \mathrm{SD} 1.15, n=12\right)$, as previously described [1]. The other contractile and relaxant agents were studied under $5 \% \mathrm{O}_{2} / 90 \% \mathrm{~N}_{2} / 5 \% \mathrm{CO}_{2}\left(\mathrm{Po}_{2} 6.96 \mathrm{kPa}, \mathrm{SD} 0.52, n=12\right)$. This level of oxygenation was chosen because it corresponds with the arterial $\mathrm{Po}_{2}$ of 15-19-day chicken embryos ( 7 kPa) [51]. Concentration-response curves to $\mathrm{KCl}(4.75 \mathrm{mM}-125 \mathrm{mM})$, the voltage-gated $\mathrm{K}^{+}$channel $(\mathrm{Kv})$ inhibitor 4aminopyridine (4-AP, $1 \mathrm{mM}-10 \mathrm{mM}$ ), the nonselective adrenoceptor agonist norepinephrine $(\mathrm{NE}, 10 \mathrm{nM}-0.1 \mathrm{mM})$, the a1-adrenoceptor agonist phenylephrine (Phe, $10 \mathrm{nM}-0.3 \mathrm{mM}$ ), the thromboxane $A_{2}$ mimetic 9,11dideoxy-11a,9a-epoxymethano-PGF $2 a$ (U46619, $10 \mathrm{nM}-10 \mu \mathrm{M})$, and endothelin-1 (ET-1, $0.01 \mathrm{nM}-0.1 \mu \mathrm{M})$, were constructed. The responses to $\mathrm{O}_{2}$, 4-AP, and $\mathrm{KCl}$ were also analyzed in DA rings from 15- and 17-day embryos. The other contractile and relaxant agents were studied only at one age (19 days). Relaxant agonists were evaluated during contraction induced by 62.5 $\mathrm{mM} \mathrm{K}$. Concentration-response curves for ACh $(10 \mathrm{nM}-30 \mu \mathrm{M})$, the nitric oxide (NO) donor sodium nitroprusside (SNP, $10 \mathrm{nM}-0.1 \mathrm{mM}$ ), the NOindependent stimulator of soluble guanylate cyclase BAY $41-2272$ (10 nM-1 $\mu \mathrm{M})$, PGE2 $(10 \mathrm{nM}-10 \mu \mathrm{M})$, the $\beta$-adrenoceptor agonist isoproterenol $(0.1 \mathrm{nM}-$ $3 \mu \mathrm{M})$, the adenyl cyclase activator forskolin $(10 \mathrm{nM}-10 \mu \mathrm{M})$, and the Rhokinase inhibitors Y-27632 $(1 \mathrm{nM}-30 \mu \mathrm{M})$ and hydroxyfasudil $(1 \mathrm{nM}-30 \mu \mathrm{M})$ were constructed. The relaxations to $\mathrm{PGE}_{2}$ were studied in the presence of the thromboxane/PGH 2 (TP) receptor antagonist SQ 29,548 $(10 \mu \mathrm{M})$, as previously described [2].

The responses to $17 \beta$-estradiol $(10 \mathrm{nM}-0.1 \mathrm{mM})$ and its stereo- isomer $17 \mathrm{a}$ estradiol $(10 \mathrm{nM}-0.1 \mathrm{mM})$ were studied in pulmonary side DA rings precontracted with $21 \% \mathrm{O}_{2}, 62.5 \mathrm{mM} \mathrm{KCl}$ (under $5 \% \mathrm{O}_{2}$ ), or $10 \mu \mathrm{M}$ phenylephrine (under $5 \% \mathrm{O}_{2}$ ) and in aortic side DA rings precontracted with $62.5 \mathrm{mM} \mathrm{KCl}$ (under $5 \% \mathrm{O}_{2}$ ). To gain insights into the putative mechanisms of estrogen-induced relaxation, some experiments were performed in endothelium-denuded vessels (endothelium was removed by gentle rubbing of 
the vessel lumen with a horse tail hair, and its absence verified by the absence of relaxation to $10 \mu \mathrm{M} \mathrm{ACh}$ ) or in the presence of one of the following pharmacological tools: the soluble guanylate cyclase (sGC) inhibitor $1 \mathrm{H}$ [1,2,4]oxadiazolo[4,3-a]quinoxalin-1-one (ODQ; $10 \mu \mathrm{M})$, the nonselective $\mathrm{K}^{+}$ channel inhibitor tetraethylammonium (TEA; $5 \mathrm{mM}$ ), the ATP-sensitive $\mathrm{K}^{+}$ channel (K $\left.\mathrm{K}_{\text {ATP }}\right)$ inhibitor glibenclamide $(10 \mu \mathrm{M})$, the large-conductance $\mathrm{Ca}^{2+-}$ activated $\mathrm{K}^{+}$channel $\left(\mathrm{BK}_{\mathrm{Ca}}\right)$ inhibitor charybdotoxin $(10 \mu \mathrm{M})$, and the estrogen receptor (ER) antagonist fulvestrant (formerly ICI 182,780, $1 \mu \mathrm{M}$ ). To test the functionality of ERs in our DA preparations, concentration-response curves to the selective ERa agonist 4,4',4"-(4-propyl-['H]pyrazole-1,3,5-triyl)tris-phenol (PPT, 0.01-10 $\mu \mathrm{M}$ ) and the selective ER $\beta$ agonist 2,3-bis(4-hydroxyphenyl)propionitrile (DPN, 0.01-10 $\mu \mathrm{M}$ ) were performed. Finally, in another set of experiments, the ability of $17 \beta$-estradiol to modulate $\mathrm{Ca}^{2+-e n t r y}$ was assessed by obtaining concentration-response curves to $\mathrm{CaCl}_{2}$. For this purpose, the $\mathrm{DA}$ rings were washed three times (5-minute interval) with $\mathrm{Ca}^{2+}$-free medium containing $1 \mathrm{mM}$ EDTA. Then, DA rings were washed twice with $\mathrm{Ca}^{2+-}$ free medium (without EDTA), incubated with vehicle, $17 \beta$-estradiol $(10 \mu \mathrm{M})$, or the L-type $\mathrm{Ca}^{2+}$ channel $\left(\mathrm{Ca}_{\mathrm{L}}\right)$ blocker nifedipine $(10 \mu \mathrm{M})$ and stimulated with $21 \%$ $\mathrm{O}_{2}$ or $\mathrm{KCl}(62.5 \mathrm{mM})$ before adding cumulative concentrations of $\mathrm{CaCl}_{2}(10$ $\mu \mathrm{M}-10 \mathrm{mM})$.

\subsubsection{Drugs and solutions}

KRB contained (in mmol/L): $118.5 \mathrm{NaCl}, 4.75 \mathrm{KCl}, 1.2 \mathrm{MgSO}_{4} \cdot 7 \mathrm{H}_{2} \mathrm{O}, 1.2$ $\mathrm{KH}_{2} \mathrm{PO}_{4}, 25.0 \mathrm{NaHCO}_{3}, 2.5 \mathrm{CaCl}_{2}$, and 5.5 glucose. Solutions containing different concentrations of $\mathrm{K}^{+}$were prepared by replacing part of the $\mathrm{NaCl}$ by an equimolar amount of $\mathrm{KCl}$. BAY 41-2272, forskolin, and ET-1 were obtained from Alexis Biochemicals (Uithoorn, The Netherlands). U46619, PGE 2 , and SQ 29,548 were obtained from Cayman Chemical (Ann Arbor, MI), and ODQ was from Tocris (Ballwin, MO). All of the other drugs were obtained from Sigma (St. Louis, MO). Drugs were initially dissolved in distilled deionized water (except forskolin and nifedipine, which were dissolved in ethanol, charybdotoxin that was dissolved in $0.9 \% \mathrm{NaCl}$, and BAY 41-2272, SQ 29,548, PGE2, U46619, estradiol, fulvestrant, PPT, and DPN that were dissolved in DMSO) to prepare stock solutions, and further dilutions were made in KRB. Total DMSO added to the organ bath for preincubations or concentration-response curves was, at the most, $0.34 \% \mathrm{vol} / \mathrm{vol}$ and did not affect arterial tone. Total ethanol was, at the most, $0.1 \% \mathrm{vol} / \mathrm{vol}$ and produced a slight contraction of the DA, as previously described [1]. 


\subsubsection{Data analysis}

Results are shown as means (SD) of measurements in n embryos. For clarity, results are shown in the figures as means $\pm \mathrm{SE}$. The contractions generated upon the addition of agonists are expressed in terms of active wall tension ( $N /$ $\mathrm{m}$ ), calculated as the force divided by twice the length of the segment, while the relaxant responses are expressed as the percentage of reduction of the precontraction. Sensitivity (expressed as $\mathrm{pD}_{2}=-\log \mathrm{EC}_{50}$ ) to agonists was determined for each artery by fitting individual concentration-response data to a nonlinear sigmoidal regression curve and interpolating (GraphPad Prism ver. 5.01; GraphPad Software, San Diego, CA). Differences between mean values were assessed by one-way ANOVA followed by Bonferroni post hoc $t$-test. Unpaired $t$-test was used if only two groups were compared. Differences were considered significant at a $P<0.05$. All analyses were performed using a commercially available statistics package (GraphPad InStat ver. 3.00).

\subsection{Results}

\subsubsection{Lack of sex-related differences in chicken DA reactivity}

The egg mass at day 0 of incubation (male: $63.75 \mathrm{~g}$, SD 4.24, $n=120$; female: $62.36 \mathrm{~g}$, SD 3.53, $n=120$ ), and the body mass of the 15-day (male: $13.15 \mathrm{~g}$, SD 1.48, $n=30$; female: $12.96 \mathrm{~g}$, SD 1.43, $\mathrm{n}=30$ ), 17-day (male: $19.58 \mathrm{~g}$, SD 1.78, $n=30$; female: $19.32 \mathrm{~g}$, SD 1.73, $n=30$ ), and 19-day (male: $29.07 \mathrm{~g}$, SD 2.11, $n=120$; female: $28.89 \mathrm{~g}$, SD 2.07, $n=120$ ) chicken embryos did not vary with sex. The chicken DA (pulmonary side) contractions induced by $\mathrm{O}_{2}$ (Fig. 7.1A), 4-AP (Fig. 7.1B), $\mathrm{KCl}$ (Fig. 7.1C), NE, Phe, U46619, and ET-1 (Fig. 7.2, $A$ and $B$ ), and the relaxations (Figs. 7.2, $C$ and $D$ ) induced by ACh, SNP, BAY 41-2272, $\mathrm{PGE}_{2}$ (in the presence of $\mathrm{SQ} 29,548$ ), isoproterenol, forskolin, Y-27632, and hydroxyfasudil were not significantly different between male and female 19-day chicken embryos. When the reactivity of the DA from 15- and 17-day embryos was analyzed, we observed a developmental increase in the contraction induced by $\mathrm{O}_{2}$ (15-day < 17-day < 19-day embryos, Fig. 7.1A), 4AP (15-day = 17-day < 19-day embryos, Fig. 7.1B), and $\mathrm{KCl}$ (15-day = 17-day $<19$-day embyros, Fig. 7.1C) but without sex-related differences. 


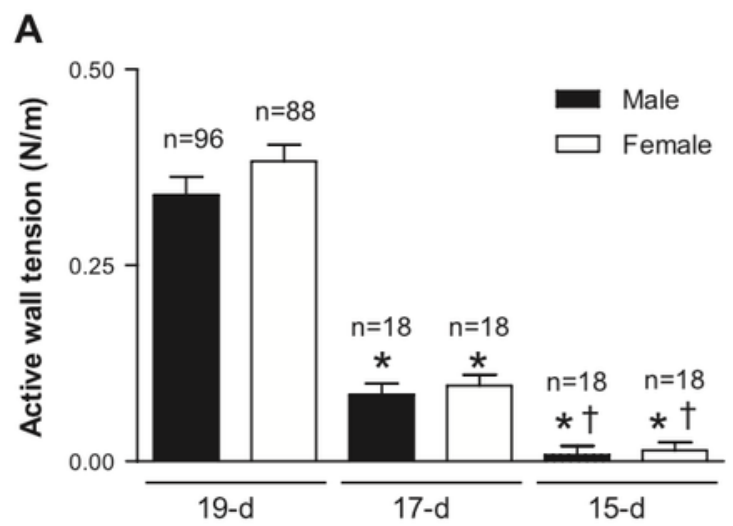

B

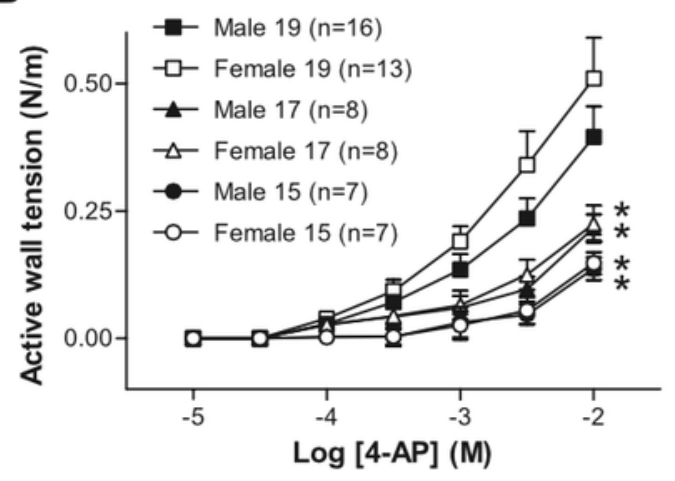

C
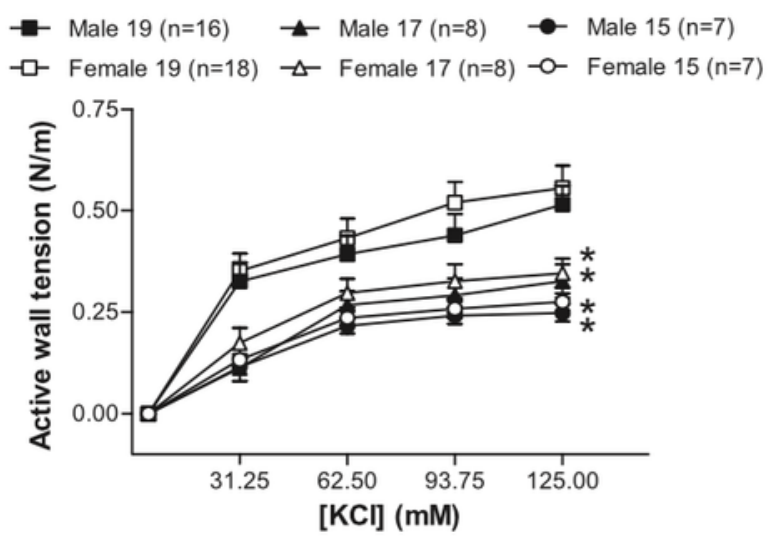

Fig. 7.1. Contractile effects of $\mathrm{O}_{2}\left(\mathrm{Po}_{2} 19 \mathrm{kPa}\right.$; $\left.A\right)$, the $\mathrm{K}_{v}$ channel inhibitor 4-aminopyridine (4-AP; $B$ ), and $\mathrm{KCl}(C)$ in ductus arteriosus (DA) rings (pulmonary side) from male and female 15-, 17-, and 19-day chicken embryos. The responses to $4-\mathrm{AP}$ and $\mathrm{KCl}$ were examined at a $\mathrm{Po}_{2}$ of $7 \mathrm{kPa}$. No sex-related differences were found. ${ }^{*} P<0.05$ for difference in maximal contraction from 19 days. $\dagger P<0.05$ for difference in maximal contraction from 17 days. 

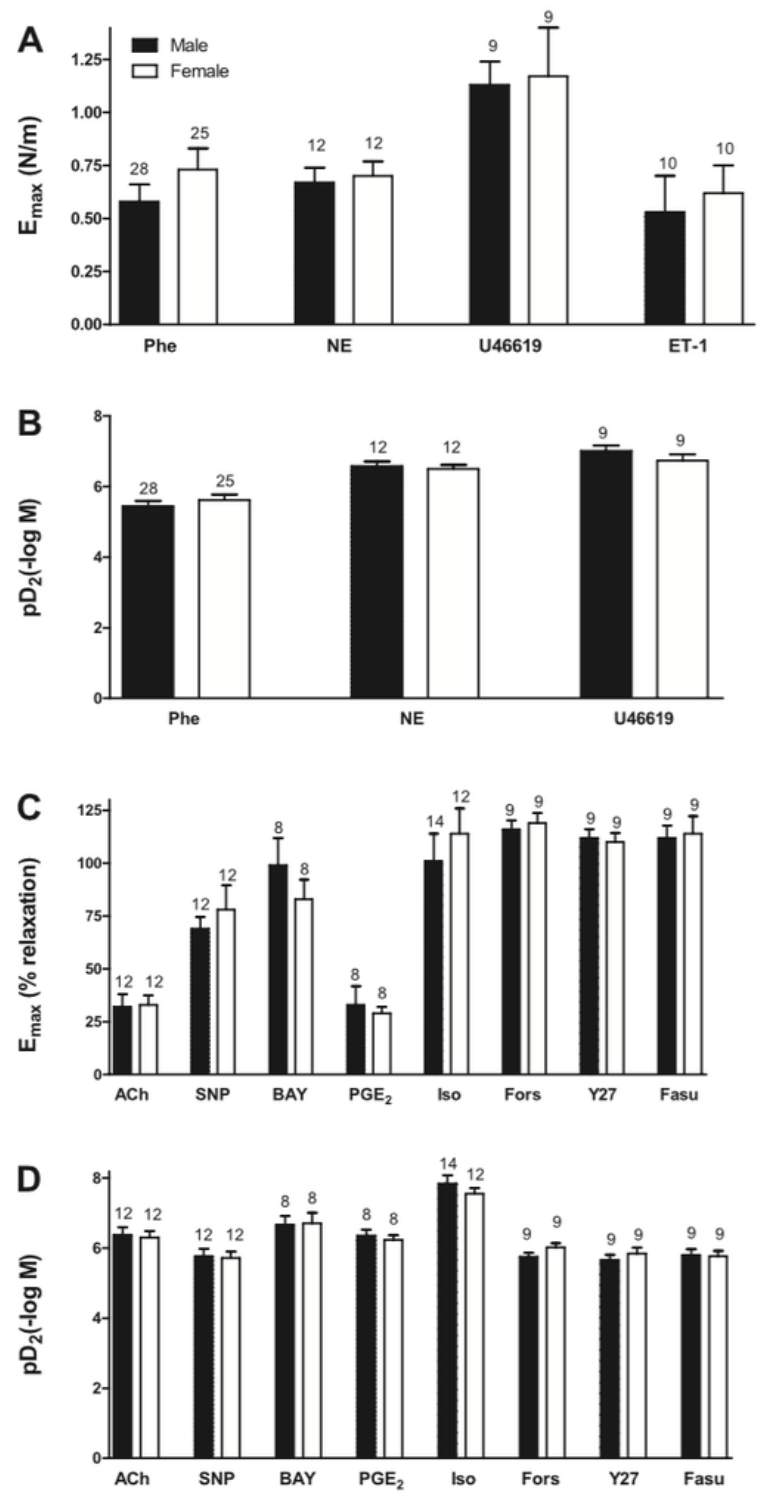

Fig. 7.2. Efficacy ( $E_{\max } ; A$ and $\left.C\right)$ and potency $\left(\mathrm{pD}_{2} ; B\right.$ and $\left.D\right)$ of the contractile agonists $(A$ and $B)$ norepinephrine (NE), phenylephrine (Phe), U46619, and endothelin-1 (ET-1), and the relaxant agonists $(C$ and $D$ ) acetylcholine (ACh), the NO donor sodium nitroprusside (SNP), the NOindependent stimulator of soluble guanylate cyclase BAY 41-2272, $\mathrm{PGE}_{2}$, the $\beta$-adrenoceptor agonist isoproterenol (Iso), the adenylate cyclase activator forskolin (Fors), and the Rho-kinase inhibitors Y-27632 (Y27) and hydroxyfasudil (Fasu) in DA rings (pulmonary side) from male and female 19-day chicken embryos. Vessels were precontracted with $62.5 \mathrm{mM} \mathrm{KCl}$. A Po 2 of $7 \mathrm{kPa}$ was maintained during the experiments. The relaxations to $P G E_{2}$ were studied in the presence of the thromboxane/PGH 2 (TP) receptor antagonist SQ 29,548. No sex-related differences were found. The numbers above the bars indicate the number of embryos studied. 


\subsubsection{Estrogen-induced relaxation of chicken $D A$}

The estrogen $17 \beta$-estradiol fully relaxed the 19-day chicken DA (pulmonary side) precontracted by $21 \% \mathrm{O}_{2}$ (Fig. $7.3, A$ and $B ; \mathrm{pD}_{2}$ male: 4.81 , SD $0.07, n=$ 6; $\mathrm{pD}_{2}$ female: $4.70, \mathrm{SD} 0.13, n=7$ ), $62.5 \mathrm{mM} \mathrm{KCl}$ (Fig. $7.3 C$, $\mathrm{pD}_{2}$ male: 4.67, SD $0.17, n=7 ; \mathrm{pD}_{2}$ female: 4.74 , SD $0.16, n=8$ ), or $10 \mu \mathrm{M}$ Phe (Fig. $7.4 A ; \mathrm{pD}_{2}$ male: 4.94, SD 0.24, $n=10 ; \mathrm{pD}_{2}$ female: 4.98 , SD 0.24, $n=8$ ). As shown in Fig. $7.3 \mathrm{D}, 17 \beta$-estradiol also induced a full relaxation of $\mathrm{KCl}$-contracted $\mathrm{DA}$ rings from the aortic side $\left(\mathrm{pD}_{2}\right.$ male: $4.81, \mathrm{SD} 0.21, n=6 ; \mathrm{pD}_{2}$ female: $4.70, \mathrm{SD}$ $0.19, n=6)$. The stereoisomer $17 a$-estradiol also relaxed chicken DA rings (pulmonary and aortic sides) with similar potency as $17 \beta$-estradiol but with a lower efficacy (Figs. 7.3 and 7.4). No sex-related differences in estrogeninduced relaxation were observed (Figs. 7.3 and 7.4).

A

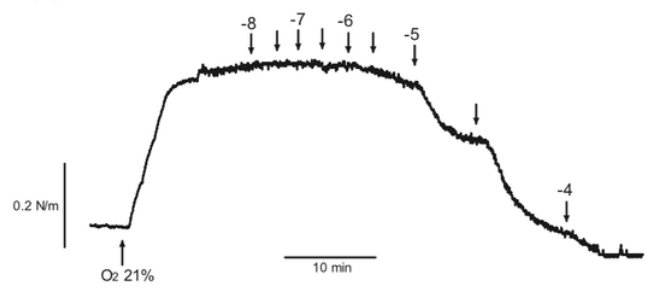

B

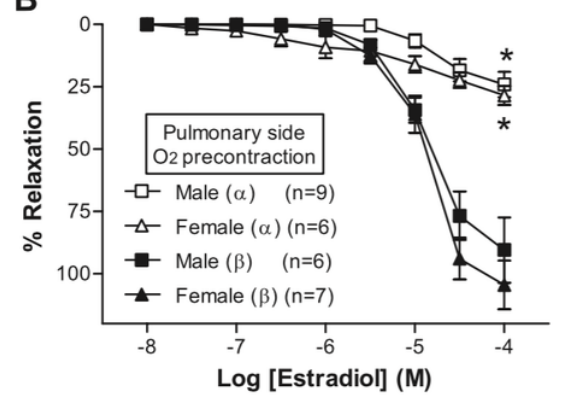

C

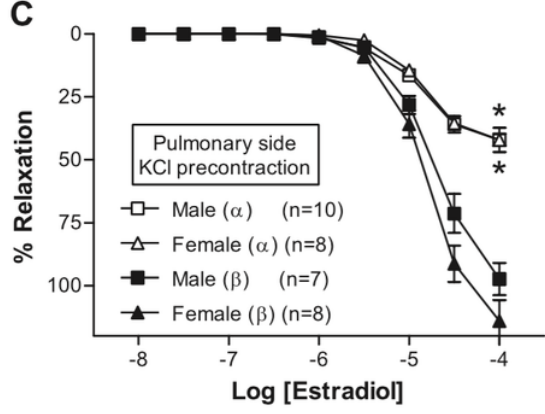

D

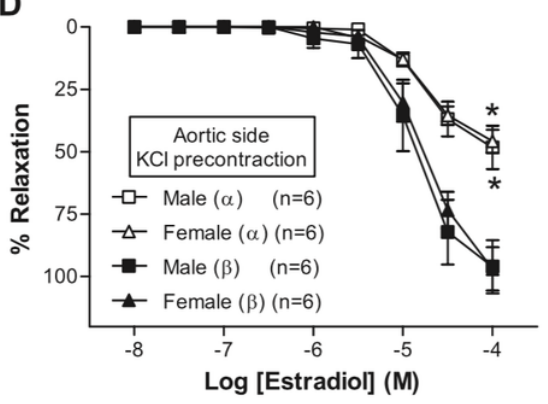

Fig. 7.3. Relaxant effects of $17 \beta$-estradiol and $17 \mathrm{a}$-estradiol in $\mathrm{O}_{2}$ - and $\mathrm{KCl}$ - contracted $\mathrm{DA}$ rings from male and female 19-day chicken embryos. $A$ : representative tracing of active wall tension vs. time showing the response of an $\mathrm{O}_{2}$-contracted DA ring to $17 \beta$-estradiol. The numbers above the tracing indicate log [estradiol] (M). $B$ : mean relaxant effects of $17 \beta$-estradiol and $17 \mathrm{a}$-estradiol in $\mathrm{O}_{2}$ contracted DA rings (pulmonary side, $\mathrm{Po}_{2} 19 \mathrm{kPa}$ ). $C$ : mean relaxant effects of $17 \beta$-estradiol and $17 a$-estradiol in $62.5 \mathrm{mM} \mathrm{KCl}$-contracted DA rings (pulmonary side, $\mathrm{Po}_{2} 7 \mathrm{kPa}$ ). $D$ : mean relaxant effects of $17 \beta$-estradiol and $17 \mathrm{a}$-estradiol in $62.5 \mathrm{mM} \mathrm{KCl}$-contracted $\mathrm{DA}$ rings (aortic side, $\mathrm{Po}_{2} 7$ $\mathrm{kPa})$. ${ }^{*} P<0.05$ for difference in maximal relaxation from $17 \beta$-estradiol. 

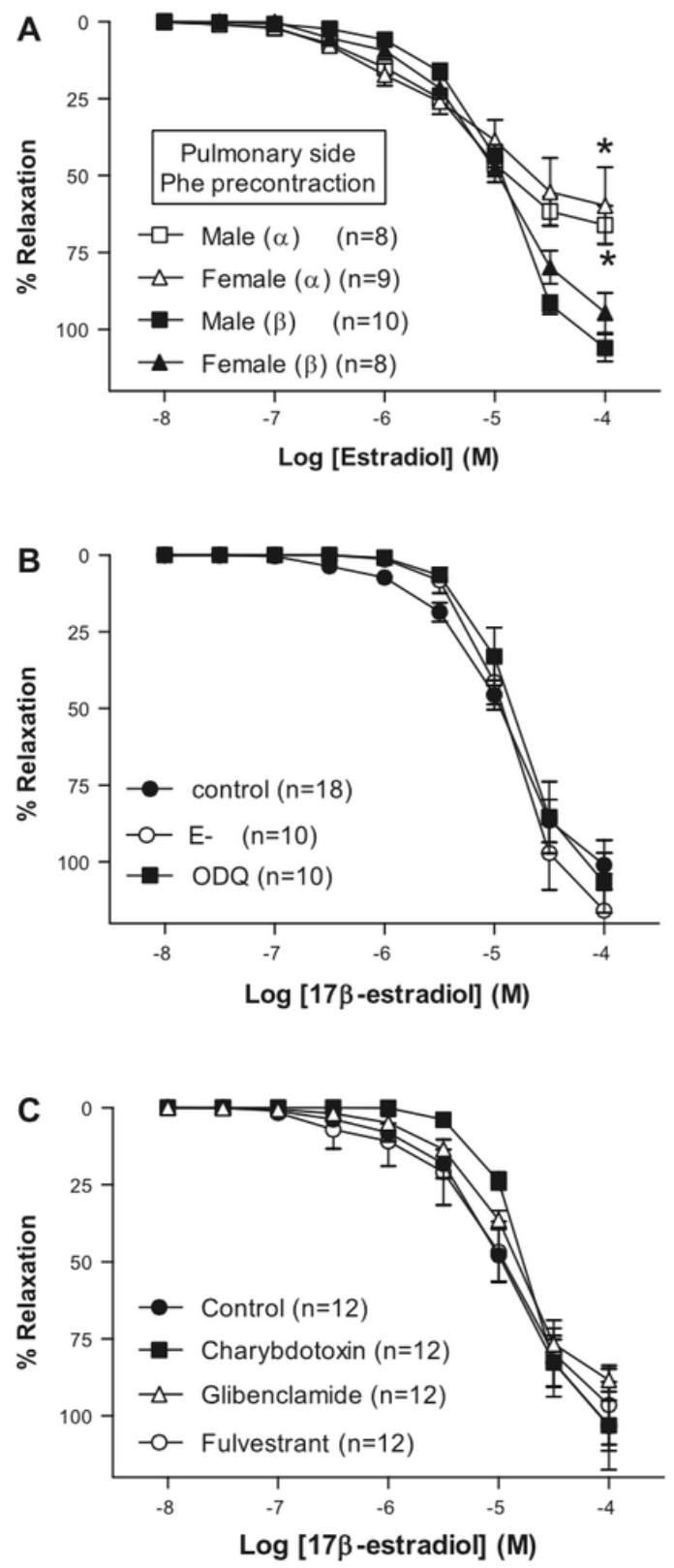

Fig. 7.4. A: Relaxant effects of $17 \beta$-estradiol and $17 \mathrm{a}$-estradiol in $10 \mu \mathrm{M}$ phenylephrine-contracted DA rings (pulmonary side, $\mathrm{Po}_{2} 7 \mathrm{kPa}$ ) from male and female 19-day chicken embryos. ${ }^{*} P<0.05$ for difference in maximal relaxation from $17 \beta$-estradiol. $B$ : lack of effect of endothelium removal (E-) or preincubation with the soluble guanylate cyclase ODQ on the relaxation induced by $17 \beta$-estradiol in chicken DA rings (pulmonary side). $C$ : lack of effect of the $\mathrm{K}_{\mathrm{Ca}}$ channel blocker charybdotoxin, the $\mathrm{K}_{\text {ATP }}$ channel blocker glibenclamide, and the estrogen receptor antagonist fulvestrant on the relaxation induced by $17 \beta$-estradiol in chicken DA rings (pulmonary side). 

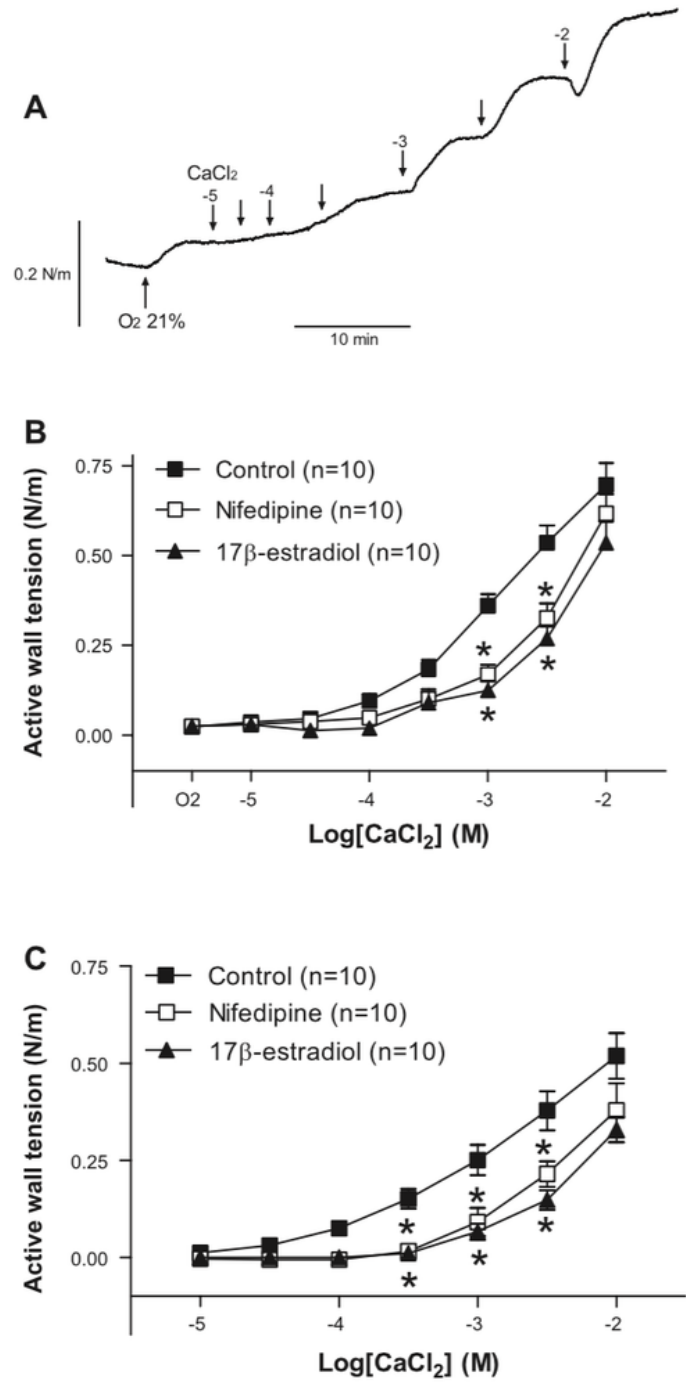

Fig. 7.5. Effects of $17 \beta$-estradiol $(10 \mu \mathrm{M})$ and the L-type $\mathrm{Ca}^{2+}$ channel blocker nifedipine $(10 \mu \mathrm{M})$ on the contraction induced by $\mathrm{CaCl}_{2}$ in 19-day chicken $\mathrm{DA}$ (pulmonary side) previously incubated in a $\mathrm{Ca}^{2+-f r e e}$ medium. $A$ : representative tracing of active wall tension vs. time showing the response of an $\mathrm{O}_{2}$-stimulated DA ring (pulmonary side) to $\mathrm{CaCl}_{2}$. The numbers above the tracing indicate $\log \left[\mathrm{CaCl}_{2}\right](\mathrm{M}) . B$ and $C$ : mean effects of $\mathrm{CaCl}_{2}$ in $\mathrm{DA}$ rings stimulated with $21 \% \mathrm{O}_{2}\left(\mathrm{Po}_{2}\right.$ $19 \mathrm{kPa}, B$ ) or $62.5 \mathrm{mM} \mathrm{KCl}\left(\right.$ at a $\mathrm{Po}_{2}$ of $\left.7 \mathrm{kPa}, C\right)$. ${ }^{*} P<0.05$ for difference from conRelaxation to $17 \beta$-estradiol was not significantly modified by mechanical rubbing of the endothelium (Fig. 7.4B) or by incubation of DA rings with the SGC inhibitor ODQ (Fig. 4B), the nonselective $\mathrm{K}^{+}$channel blocker TEA (not shown), the selective $\mathrm{K}_{\mathrm{Ca}}$ channel blocker charybdotoxin (Fig. 7.4C), the selective $K_{\text {ATP }}$ channel blocker glibenclamide (Fig. 7.4C), or the ER antagonist fulvestrant (Fig. 7.4C). The selective ERa agonist PPT and the selective ER $\beta$ agonist DPN did not induce relaxation of phenylephrine-contracted DA rings (not shown). 
Cumulative concentrations of $\mathrm{CaCl}_{2}(10 \mu \mathrm{M}-10 \mathrm{mM})$ induced concentrationdependent contraction in DA rings (pulmonary side) stimulated with $21 \% \mathrm{O}_{2}$ or depolarized by $62.5 \mathrm{mM} \mathrm{KCl}$ (under $5 \% \mathrm{O}_{2}$ ) in $\mathrm{Ca}^{2+}$-free medium (Fig. 7.5). As shown in Fig. $7.5 \mathrm{~A}$ and previously described (13), $\mathrm{O}_{2}$ induced a slight contraction $(0.025 \mathrm{~N} / \mathrm{m}, \mathrm{SD} 0.002, n=10)$ in the absence of extracellular $\mathrm{Ca}^{2+}$. Preincubation with $17 \beta$-estradiol $(10 \mu \mathrm{M})$ or the L-type $\mathrm{Ca}^{2+}$ channel blocker nifedipine $(10 \mu \mathrm{M})$ produced a partial inhibition of $\mathrm{CaCl}_{2}$-induced contraction (Fig. 7.5, $B$ and $C$ ).

\subsection{Discussion}

The participation of different sex hormones on vascular tone regulation has been widely described [38]. To our knowledge, however, the present study is the first attempt to evaluate sex-related differences in DA reactivity and DA responsiveness to estrogens in any species. We did not observe significant sex-related differences in chicken DA response to contractile or relaxant stimuli but demonstrated that $17 \beta$-estradiol elicits an endothelium and estrogen receptor-independent relaxation that appears to be mediated, at least partially, by the inhibition of $\mathrm{Ca}^{2+}$ entry from extracellular space.

\subsubsection{Absence of sex-related differences in DA reactivity}

In the last two years, the vascular biology of the chicken DA has been characterized by our group and another laboratory. The chicken DA responds to a wide variety of vasoconstrictors and vasodilators and undergoes, in general, a developmental increase in its responsiveness to constrictors and a developmental decrease in its responsiveness to relaxant agonists [1-3]. Similarly to the mammalian DA, closure of the chicken DA occurs in two stages: an initial occlusion, mediated by constriction of smooth muscle in the pulmonary side, followed by anatomical vessel remodeling, leading to luminal obliteration [4]. The timing of the closure of the DA correlates with an increased contractile response of the vessel to $\mathrm{O}_{2}$, and the mechanisms underlying $\mathrm{O}_{2}$ sensing and signaling appear to be very similar in chickens and mammals $[1,4,13,21]$. In contrast, the main vasodilator of the mammalian $D A, P E_{2}$, is a weak relaxant agent of the chicken DA, which even induces contraction through TP receptor stimulation [2,21].

Herein, we report that there are no sex-related differences in DA reactivity. However, it should be noted that the majority of our study was performed in 19day chicken embryos. The 19-day chicken embryo is close to initiating the 
transition to ex ovo life (i.e., internal pipping) and presents, therefore, a relatively "mature" DA $[1-4,13,21]$. For that reason, we decided to investigate whether sex-related differences in DA reactivity were present in DAs from less mature chicken embryos (i.e., 15- and 17-day embryos). We confirmed that although the 15-day DA contracts in response to high $\mathrm{K}^{+}$-depolarizing solution and $\mathrm{K}_{\mathrm{v}}$ channel inhibition, it is almost unresponsive to $\mathrm{O}_{2}[1,13]$. In contrast, $\mathrm{O}_{2}$ elicited a tonic contraction in the 17-day DA that was $\sim 25 \%$ of the contraction observed in the 19-day DA. The reactivity of 15-day and 17-day DAs was similar between males and females, suggesting that the developmental trajectory of the DA is not sex specific. Therefore, our first hypothesis, i.e., the presence of sexual dimorphism in chicken DA reactivity, was not supported by our results. It could be argued that the chicken is not a good model for studying sex-related differences in cardiovascular (patho)physiology. The information about sexual dimorphism in chicken cardiovascular (patho)physiology is scarce when compared with the data available from mammalian species. However, it has been demonstrated that male chickens are more prone to show elevation of blood pressure, neointimal plaque formation in the abdominal aorta, and aorta hardening than females $[35,44]$. In addition, male chickens present a higher incidence of pulmonary hypertension [60] and heart failure [37] than females. Although these alterations do not take place in early life and a direct role for sex hormones has not been demonstrated, the above data suggest that the chicken is a species in which sex hormones might influence cardiovascular development.

\subsubsection{Responsiveness to estrogen of chicken $D A$}

Our second hypothesis, i.e., that estrogens are vasoactive in the chicken DA, was supported by our results. However, it should be noted that chicken DA relaxation was induced with concentrations of estrogen far greater than the high picomolar-nanomolar levels of free hormone found in the plasma of chicken embryos at this developmental stage [56]. In many mammalian studies, vascular effects of estrogen in vitro require $\sim 1,000$ times higher concentrations of free hormone than are typically present in circulating plasma [57]. Therefore, effects of micromolar estrogen in vitro are often considered as mere pharmacological curiosities rather than potentially relevant responses [57]. Nevertheless, it has been argued that free plasma levels of estrogen may far underestimate actual effective concentrations in the vicinity and inside target cells and that estrogen can act in a paracrine or even autocrine manner in vascular smooth cells [24,57]. Thus, as proposed by White [57], it appears no longer accurate to assume that only responses to nanomolar concentrations of 
estrogen have physiological relevance.

Numerous studies demonstrated that the response and the specific mechanisms responsible for the vascular effects of estrogens are species, age, sex, and vessel dependent [10,11,16,22,23,25,29,33,41-43,45,49,57-59). Several studies strongly support that estrogen-evoked relaxation is mediated via endothelium-derived $\mathrm{NO}$, but endothelium-derived prostacyclin has also been proposed as a mediator [10,16,27,38,41,43,45,48,49,57]. Other authors demonstrated an endothelium-independent effect of estrogen through activation of neuronal NOS, or by modulation of $\mathrm{Ca}^{2+}$ and/or $\mathrm{K}^{+}$channel activity [11,22,23, $29,33,42,43,57-59]$. By using pharmacological tools, we evaluated the participation of some of these mechanisms in the ductal effects of estrogen. We observed that estrogen-mediated DA relaxation was endothelium independent and was unaffected by the presence of the SGC inhibitor ODQ, or the $\mathrm{K}^{+}$ channel blockers TEA (nonselective), glibenclamide (KATP selective), or charybdotoxin ( $\mathrm{BK}_{\mathrm{Ca}}$ selective). Therefore, three of the mechanisms most frequently involved in estrogen-induced relaxation of mammalian vessels (i.e., the activation of the NO-sGC-cyclic GMP pathway and the opening of $\mathrm{BK}_{\mathrm{Ca}}$ or $K_{\text {ATP }}$ channels) [57-59] appear not to be involved in the chicken DA relaxation. The majority of data from mammalian studies indicates that estrogen lowers intracellular $\mathrm{Ca}^{2+}$ concentration in vascular smooth muscle cells [57]. One study indicated that estrogen may reduce sarcoplasmic reticulum $\mathrm{Ca}^{2+}$ release [23], but other investigators have found that estrogen-induced vascular relaxation does not involve significant $\mathrm{Ca}^{2+}$ release from intracellular stores or changes in $\mathrm{Ca}^{2+}$ sensitivity of the contractile apparatus [15,30,57]. Rather, estrogen attenuates extracellular $\mathrm{Ca}^{2+}$ influx $[15,57]$ and/or stimulates $\mathrm{Ca}^{2+}$ efflux [40]. The inhibition of extracellular $\mathrm{Ca}^{2+}$ influx by $17 \beta$-estradiol is supported by our results showing inhibition of the contraction elicited by $\mathrm{CaCl}_{2}$ in $\mathrm{O}_{2-}$ or $\mathrm{KCl}$-stimulated chicken $\mathrm{DA}$ rings. These data raise functional evidence for the $\mathrm{Ca}^{2+}$ antagonistic effect of $17 \beta$-estradiol in chicken DA and are in line with a similar effect reported in rabbit carotid [46], cerebral [47], and coronary [28] arteries. In addition, electrophysiological evidence of inhibition of $\mathrm{Ca}^{2+}$ currents by $17 \beta$-estradiol has been reported in several mammalian vascular smooth muscle cells $[36,62]$. Interestingly, we observed that when $17 \beta$-estradiol was compared with the dihydropyridine nifedipine (a classic CaL channel blocker), both compounds showed, at high micromolar concentrations, a similar functional antagonism against $\mathrm{CaCl}_{2}$-induced contraction. In contrast to our results, dihydropyridines showed a higher $\mathrm{Ca}^{2+}$ antagonistic potency than $17 \beta$-estradiol in mammalian vessels $[46,47]$. Compared with other chicken vascular beds (like the pulmonary arteries, authors' unpublished 
observations), the DA appears to be less sensitive to dihydropyridines as evidenced by the low potencies of the CaL agonist BAY K8644 to induce contraction and of nifedipine to relax $\mathrm{KCl}$ - or $\mathrm{O}_{2}$-induced contraction [13]. However, it should be noted that, although an important part of the normoxic DA contraction relies on $\mathrm{Ca}^{2+}$ entry through $\mathrm{Ca}_{L}$ channels, inhibition of $\mathrm{O}_{2-}$ induced contraction in human [31] or lamb [12,55] DA also required relatively high concentrations of nifedipine $(10 \mu \mathrm{M})$.

\subsubsection{Estrogens and the two sides of chicken DA}

An extremely interesting feature of the chicken DA is the presence of morphological and functional heterogeneity along its path between the pulmonary artery and the aorta. Thus, the pulmonary side shows the structure of a muscular artery and responds to $\mathrm{O}_{2}$ with contraction, whereas the aortic part shows the morphology of an elastic artery and relaxes in response to $\mathrm{O}_{2}$ $[1,4,14]$. In addition, the two parts of the DA show marked differences in their responsiveness to contractile and relaxant agents [2,3]. Bergwerff et al. [5] demonstrated that the distal elastic part of the chicken DA is mesodermal in origin and is the result of the incorporation of dorsal aorta tissue, whereas the muscular pulmonary side was shown to consist almost exclusively of neural crest-derived cells. Interestingly, estrogen methylation rate appears to be different in vascular smooth muscle cells depending on the mesodermal or the neural crest origin [61]. However, in the present work, we observed that estrogen-induced relaxation was uniformly present in both segments of the chicken DA, suggesting a similar sensitivity to estrogen in these two populations of vascular smooth muscle cells.

\subsubsection{Estrogen receptors and estrogen-induced relaxation of chicken $D A$}

Numerous studies demonstrate that mammalian vascular endothelium and smooth muscle cells express both ERa and ER $\beta$, but this expression seems heterogeneous with regard to species, vascular bed, and sex [57]. Herein, we observed that chicken DA relaxation induced by $17 \beta$-estradiol was not affected by the presence of the ER antagonist fulvestrant, and neither the specific ERa agonist PPT nor the specific ER $\beta$ agonist DPN showed any relaxant effect in the chicken DA. These considerations lead us to postulate that $17 \beta$-estradiol induces endothelium-independent relaxation of chicken DA via a mechanism that does not require the participation of the classical estrogen receptors. However, it shows stereospecificity because 17a-estradiol also relaxed the chicken DA with similar potency but lower efficacy. This stereospecificity may indicate that a putative, receptive, specific structure is responsible for the 
relaxant effect. It has been suggested that estrogens could act through direct binding to regulatory subunits in the CaL channel [47]. Alternatively, other receptors for the nongenomic actions of estrogens, such as seventransmembrane $\mathrm{G}$ protein-coupled receptor 30 [48], have been proposed.

\subsubsection{Perspectives and significance}

The mammalian placenta provides a highly estrogenic environment for both female and male fetuses. In contrast, in ovo development of the chicken takes place under a marked sex-related difference in estrogen levels $[6,9,20]$. Our present data do not suggest that such a difference influences DA reactivity. However, the chicken embryo still appears as a suitable natural model to study the influence of early estrogen exposure in other vascular beds, like the coronary circulation, particularly susceptible to the hormone. In addition, manipulation of the sex steroid hormonal environment of the chicken embryo can be easily performed by injection of estrogens or inhibitors of estrogen synthesis into the albumen of fertilized eggs $[6,9,20]$. Thus, a single treatment with an aromatase inhibitor, which blocks the synthesis of estrogen from testosterone, at an embryonic stage when gonads are bipotential, causes genetic females to develop a permanent male phenotype [20]. On the contrary, the embryos that are exposed to estradiol in ovo hatch as phenotypic females regardless of their genotypic sex [6,9]. Whether these hormonal manipulations influence developmental vascular biology warrants further investigation. 


\section{References}

1. Agren P, Cogolludo AL, Kessels CG, Perez-Vizcaino F, De Mey JG, Blanco CE, Villamor E. Ontogeny of chicken ductus arteriosus response to oxygen and vasoconstrictors. Am J Physiol Regul Integr Comp Physiol 292: R485-R496, 2007.

2. Agren P, van der Sterren S, Cogolludo AL, Blanco CE, Villamor E. Developmental changes in the effects of prostaglandin E2 in the chicken ductus arteriosus. J Comp Physiol 179: 133-143, 2009.

3. Agren P, van der Sterren S, Cogolludo AL, Frazziano G, de Mey JG, Blanco CE, Villamor E. Developmental changes in endothelium-depen- dent relaxation of the chicken ductus arteriosus. J Physiol Pharmacol 59: 55-76, 2008.

4. Belanger C, Copeland J, Muirhead D, Heinz D, Dzialowski EM. Morphological changes in the chicken ductus arteriosi during closure at hatching. Anat Rec 291: 1007-1015, 2008.

5. Bergwerff M, DeRuiter MC, Poelmann RE, Gittenberger-de Groot AC. Onset of elastogenesis and downregulation of smooth muscle actin as distinguishing phenomena in artery differentiation in the chick embryo. Anat Embryol 194: 545557, 1996.

6. Biau S, Bayle S, de Santa Barbara P, Roig B. The chick embryo: an animal model for detection of the effects of hormonal compounds. Anal Bioanal Chem 387: 1397-1403, 2007.

7. Bjornstrom L, Sjoberg M. Mechanisms of estrogen receptor signaling: convergence of genomic and nongenomic actions on target genes. Mol Endocrinol 19: 833-842, 2005.

8. Brothwood M, Wolke D, Gamsu H, Benson J, Cooper D. Prognosis of the very low birthweight baby in relation to gender. Arch Dis Child 61: 559 -564, 1986.

9. Bruggeman V, Van As P, Decuypere E. Developmental endocrinology of the reproductive axis in the chicken embryo. Comp Biochem Physiol 131: 839 -846, 2002.

10. Caulin-Glaser T, Garcia-Cardena G, Sarrel P, Sessa WC, Bender JR. 17ß-estradiol regulation of human endothelial cell basal nitric oxide release, independent of cytosolic Ca 2+ mobilization. Circ Res 81: 885-892, 1997

11. Chester AH, Jiang $\mathrm{C}$, Borland JA, Yacoub MH, Collins P. Oestrogen relaxes human epicardial coronary arteries through non-endothelium-dependent mechanisms. Coron Artery Dis 6: 417-422, 1995.

12. Clyman RI, Waleh N, Kajino H, Roman C, Mauray F. Calcium- dependent and calcium-sensitizing pathways in the mature and immature ductus arteriosus. Am J Physiol Regul Integr Comp Physiol 293: R1650 - R1656, 2007.

13. Cogolludo AL, Moral-Sanz J, Van der Sterren S, Frazziano G, van Cleef AN, Menendez C, Zoer B, Moreno E, Roman A, Perez-Vizcaino F, Villamor E. Maturation of $\mathrm{O} 2$ sensing and signalling in the chicken ductus arteriosus. Am J Physiol Lung Cell Mol Physiol 297: L619 -L630, 2009. 
14. Copeland J, Dzialowski EM. Effects of hypoxic and hyperoxic incubation on the reactivity of the chicken embryo (Gallus gallus) ductus arteriosi in response to catecholamines and oxygen. Exp Physiol 94: 152-161, 2009.

15. Crews JK, Khalil RA. Gender-specific inhibition of Ca2+ entry mecha- nisms of arterial vasoconstriction by sex hormones. Clin Exp Pharmacol Physiol 26: 707715, 1999.

16. Darkow DJ, Lu L, White RE. Estrogen relaxation of coronary artery smooth muscle is mediated by nitric oxide and cGMP. Am J Physiol Heart Circ Physiol 272: H2765-H2773, 1997.

17. Denton K, Baylis C. Physiological and molecular mechanisms governing sexual dimorphism of kidney, cardiac, and vascular function. Am J Physiol Regul Integr Comp Physiol 292: R697-R699, 2007.

18. Di Renzo GC, Rosati A, Sarti RD, Cruciani L, Cutuli AM. Does fetal sex affect pregnancy outcome? Gend Med 4: 19 -30, 2007.

19. Drevenstedt GL, Crimmins EM, Vasunilashorn S, Finch CE. The rise and fall of excess male infant mortality. Proc Natl Acad Sci USA 105: 5016 -5021, 2008.

20. Elbrecht $A$, Smith RG. Aromatase enzyme activity and sex determination in chickens. Science 255: 467-470, 1992.

21. Greyner H, Dzialowski EM. Mechanisms mediating the oxygen-induced vasoreactivity of the ductus arteriosus in the chicken embryo. Am J Physiol Regul Integr Comp Physiol 295: R1647-R1659, 2008.

22. Han G, Ma H, Chintala R, Miyake K, Fulton DJ, Barman SA, White RE. Nongenomic, endothelium-independent effects of estrogen on human coronary smooth muscle are mediated by type I (neuronal) NOS and PI3-kinase-Akt signaling. Am J Physiol Heart Circ Physiol 293: H314-H321, 2007.

23. Han SZ, Karaki H, Ouchi Y, Akishita M, Orimo H. 17p-Estradiol inhibits Ca2+ influx and $\mathrm{Ca} 2+$ release induced by thromboxane $\mathrm{A} 2$ in porcine coronary artery. Circulation 91: 2619-2626, 1995.

24. Harada N, Sasano H, Murakami H, Ohkuma T, Nagura H, Takagi Y. Localized expression of aromatase in human vascular tissues. Circ Res 84: 1285-1291, 1999.

25. Haynes MP, Li L, Russell KS, Bender JR. Rapid vascular cell responses to estrogen and membrane receptors. Vasc Pharmacol 38: 99 -108, 2002.

26. Ho KJ, Liao JK. Nonnuclear actions of estrogen. Arterioscler Thromb Vasc Biol 22: 1952-1961, 2002.

27. Huang A, Sun D, Koller A, Kaley G. Gender difference in flow-induced dilation and regulation of shear stress: role of estrogen and nitric oxide. Am $\mathrm{J}$ Physiol Regul Integr Comp Physiol 275: R1571-R1577, 1998.

28. Jiang CW, Sarrel PM, Lindsay DC, Poole-Wilson PA, Collins P. Endotheliumindependent relaxation of rabbit coronary artery by $17 \mathrm{p}$ - oestradiol in vitro. $\mathrm{Br} \mathrm{J}$ Pharmacol 104: 1033-1037, 1991.

29. Keung W, Vanhoutte PM, Man RY. Acute impairment of contractile responses by $17 p$-estradiol is cAMP and protein kinase G dependent in vascular smooth muscle 
cells of the porcine coronary arteries. Br J Pharmacol 144: 71-79, 2005.

30. Kitazawa T, Hamada E, Kitazawa K, Gaznabi AK. Non-genomic mechanism of 17poestradiol-induced inhibition of contraction in mam- malian vascular smooth muscle. J Physiol 499: 497-511, 1997.

31. Michelakis E, Rebeyka I, Bateson J, Olley P, Puttagunta L, Archer S. Voltage-gated potassium channels in human ductus arteriosus. Lancet 356: 134-137, 2000.

32. Moonen RMJ, Agren P, Cogolludo AL, Perez-Vizcaino F, Villamor E. Response of chicken ductus arteriosus to hypercarbic and normocarbic acidosis. Neonatology 98: 47-56, 2009.

33. Mugge A, Riedel M, Barton M, Kuhn M, Lichtlen PR. Endothelium independent relaxation of human coronary arteries by $17 \mathrm{p}$-oestradiol in vitro. Cardiovasc Res 27: 1939-1942, 1993.

34. Naeye RL, Burt LS, Wright DL, Blanc WA, Tatter D. Neonatal mortality, the male disadvantage. Pediatrics 48: 902-906, 1971.

35. Nishimura H, Xi Z, Zhang L, Kempf H, Wideman RF, Corvol P. Maturationdependent neointima formation in fowl aorta. Com Biochem Physiol 130: 39 -54, 2001.

36. Ogata R, Inoue $\mathrm{Y}$, Nakano H, Ito $\mathrm{Y}$, Kitamura K. Oestradiol-induced relaxation of rabbit basilar artery by inhibition of voltage-dependent $\mathrm{Ca}$ channels through GTPbinding protein. Br J Pharmacol 117: 351-359, 1996.

37. Olkowski AA. Pathophysiology of heart failure in broiler chickens: structural, biochemical, and molecular characteristics. Poult Sci 86: 999 - 1005, 2007.

38. Orshal JM, Khalil RA. Gender, sex hormones, and vascular tone. Am J Physiol Regul Integr Comp Physiol 286: R233-R249, 2004.

39. Pepine CJ, Nichols WW, Pauly DF. Estrogen and different aspects of vascular disease in women and men. Circ Res 99: 459 -461, 2006.

40. Prakash YS, Togaibayeva AA, Kannan MS, Miller VM, Fitzpatrick LA, Sieck GC. Estrogen increases $\mathrm{Ca2}+$ efflux from female porcine coronary arterial smooth muscle. Am J Physiol Heart Circ Physiol 276: H926 -H934, 1999.

41. Rahimian R, Chan L, Goel A, Poburko D, van Breemen C. Estrogen modulation of endothelium-derived relaxing factors by human endothelial cells. Biochem Biophys Res Commun 322: 373-379, 2004.

42. Rosenfeld CR, Cornfield DN, Roy T. Ca2+-activated K+ channels modulate basal and $E(2) p$-induced rises in uterine blood flow in ovine pregnancy. Am J Physiol Heart Circ Physiol 281: H422-H431, 2001.

43. Rosenfeld CR, White RE, Roy T, Cox BE. Calcium-activated potassium channels and nitric oxide coregulate estrogen-induced vasodilation. Am J Physiol Heart Circ Physiol 279: H319-H328, 2000.

44. Ruiz-Feria CA, Yang Y, Thomason DB, White J, Su G, Nishimura H. Pulse wave velocity and age- and gender-dependent aortic wall hardening in fowl. Comp Biochem Physiol 154: 429 -436, 2009.

45. Salhab WA, Shaul PW, Cox BE, Rosenfeld CR. Regulation of types I and III NOS 
in ovine uterine arteries by daily and acute estrogen exposure. Am J Physiol Heart Circ Physiol 278: H2134 -H2142, 2000.

46. Salom JB, Burguete MC, Perez-Asensio FJ, Centeno JM, Torregrosa G, Alborch E. Acute relaxant effects of $17-p$-estradiol through non- genomic mechanisms in rabbit carotid artery. Steroids 67: $339-346,2002$.

47. Salom JB, Burguete MC, Perez-Asensio FJ, Torregrosa G, Alborch E. Relaxant effects of $17 \beta$-estradiol in cerebral arteries through Ca2+ entry inhibition. J Cereb Blood Flow Metab 21: 422-429, 2001.

48. Scott PA, Tremblay A, Brochu M, St-Louis J. Vasorelaxant action of 17-estradiol in rat uterine arteries: role of nitric oxide synthases and estrogen receptors. J Physiol Heart Circ Physiol 293: H3713-H3719, 2007.

49. Sherman TS, Chambliss KL, Gibson LL, Pace MC, Mendelsohn ME, Pfister SL, Shaul PW. Estrogen acutely activates prostacyclin synthesis in ovine fetal pulmonary artery endothelium. Am J Respir Cell Mol Biol 26: 610 -616, 2002.

50. Stevenson DK, Verter J, Fanaroff AA, Oh W, Ehrenkranz RA, Shankaran S, Donovan EF, Wright LL, Lemons JA, Tyson JE, Korones SB, Bauer CR, Stoll BJ, Papile LA. Sex differences in outcomes of very low birthweight infants: the newborn male disadvantage. Arch Dis Child 83: F182-F185, 2000.

51. Tazawa H, Visschedijk AH, Wittmann J, Piiper J. Gas exchange, blood gases and acid-base status in the chick before, during and after hatching. Respir Physiol 53: 173-185, 1983.

52. Vaillant S, Dorizzi M, Pieau C, Richard-Mercier N. Sex reversal and aromatase in chicken. J Exp Zool 290: 727-740, 2001.

53. Van der Sterren S, Agren P, Zoer B, Kessels L, Blanco CE, Villamor E. Morphological and functional alterations of the ductus arteriosus in a chicken model of hypoxia-induced fetal growth retardation. Pediatr Res 65: 279 -284, 2009.

54. Villamor E, Ruijtenbeek K, Pulgar V, De Mey JG, Blanco CE. Vascular reactivity in intrapulmonary arteries of chicken embryos during transition to ex ovo life. Am J Physiol Regul Integr Comp Physiol 282: R917-R927, 2002.

55. Waleh N, Reese J, Kajino H, Roman C, Seidner S, McCurnin D, Clyman RI. Oxygen-induced tension in the sheep ductus arteriosus: effects of gestation on potassium and calcium channel regulation. Pediatr Res 65: 285-290, 2009.

56. Weniger JP, Chouraqui J, Zeis A. 17ß-estradiol secretion in normal and hypophysectomized chick embryos. Reprod Nutr Develop 29: 89 -93, 1989.

57. White RE. Estrogen and vascular function. Vasc Pharmacol 38: 73-80, 2002.

58. White RE, Darkow DJ, Lang JL. Estrogen relaxes coronary arteries by opening BKCa channels through a cGMP-dependent mechanism. Circ Res 77: 936 -942, 1995.

59. White RE, Han G, Maunz M, Dimitropoulou C, El-Mowafy AM, Barlow RS, Catravas JD, Snead C, Carrier GO, Zhu S, Yu X. Endothelium-independent effect of estrogen on Ca2+-activated $\mathrm{K}+$ chan- nels in human coronary artery smooth muscle cells. Cardiovasc Res 53: 650 -661, 2002. 
60. Wideman RF Jr, French $\mathrm{H}$. Ascites resistance of progeny from broiler breeders selected for two generations using chronic unilateral pulmonary artery occlusion. Poult Sci 79: $396-401,2000$.

61. Zacharia LC, Jackson EK, Gillespie DG, Dubey RK. Increased 2-methoxyestradiol production in human coronary versus aortic vascular cells. Hypertension 37: 658 662, 2001.

62. Zhang F, Ram JL, Standley PR, Sowers JR. 17p-Estradiol attenuates voltagedependent $\mathrm{Ca} 2+$ currents in A7r5 vascular smooth muscle cell line. Am J Physiol Cell Physiol 266: C975-C980, 1994. 
Chapter 8

\section{Summary, general discussion and future perspectives}




\subsection{The chicken as a model for ductus arteriosus developmental biology}

According to the American philosopher Marx Wartofsky, "anything can be a model of anything else! This is to say no more than that between any two things in the universe there is some property they both share" (1). Therefore, models do not have to resemble the thing being modeled in every respect. Indeed, in some cases it is essential for the model to be different from the thing being modeled (2). Chickens are warm-blooded vertebrates and share most of their biology with mammals but, being evolutionarily distinct, they often do the same things in ways that are subtly different. It is these differences that have made chickens a fruitful source of discovery in a wide range of biological processes (3). For example, it was in the chicken that B cells and the tumorigenic properties of viruses were first discovered. During the past decade, various chick-based experimental systems have continued to lead to discoveries of profound biological significance (3).

The studies collected in the present thesis, together with other publications of our (4-8) and Dzialowski's (9-11) groups, show that the chicken DA is much more than a biological rarity only interesting to comparative physiologists. The chicken is an ideal model for the study of new hypotheses on developmental biology of the DA and other blood vessels (12-21) and, particularly, for the investigation of vascular oxygen sensing mechanisms. A unique characteristic of chicken DA is that it is the result of the fusion of two vessels with different embryological origin, morphology and functionality. The pulmonary side (PulmDA) consists almost exclusively of neural crest derived cells, shows the structure of a muscular artery and responds to $\mathrm{O}_{2}$ with contraction, whereas the aortic part (AoDA) is of mesodermal origin, shows the morphology of an elastic artery and relaxes in response to $\mathrm{O}_{2}(5,10-12,22,23)$. The two parts of the DA show marked differences in responsiveness not only to $\mathrm{O}_{2}$ but to other contractile and relaxant agents $(5-8,24)$ as well. These differences were summarized in table 1.1 of chapter 1 . The responses of PulmDA and AoDA to the vasoactive agents that have been studied in the present thesis are summarized in table 8.1 of the present chapter. Another advantage of the chicken DA model is that we have an exhaustive developmental calendar. The developmental changes in PulmDA responsiveness to contractile and relaxant agents were summarized in table 1.2 of chapter 1 . The developmental changes in the responsiveness to the vasoactive agents that have been studied in the present thesis are summarized in table 8.2 of the present chapter. 
Table 8.1 Effect of the vasoactive mediators investigated in the present thesis in the pulmonary (PulmDA) and the aortic (AoDA) side of chicken DA.

\begin{tabular}{|l|l|l|l|l|l|}
\hline $\begin{array}{l}\text { Vasoactive } \\
\text { agent }\end{array}$ & $\begin{array}{l}\text { Nature or mechanism } \\
\text { of action }\end{array}$ & $\begin{array}{l}\text { Effect in } \\
\text { PulmDA }\end{array}$ & $\begin{array}{l}\text { Effect in } \\
\text { AoDA }\end{array}$ & Chapter \\
\hline $15-\mathrm{E}_{2 t}$-IsoP & Isoprostane & Contraction & $>$ & Contraction & 6 \\
\hline $15-\mathrm{F}_{2 t}$-IsoP & Isoprostane & Contraction & $>$ & Contraction & 6 \\
\hline $\mathrm{H}_{2} \mathrm{O}_{2}$ & Reactive oxygen species & Contraction & $\neq$ & Relaxation & 4 \\
\hline $\mathrm{Hydroxyfasudil}$ & Rho-kinase inhibitor & Relaxation & $=$ & Relaxation & 2,3 \\
\hline Y27632 & Rho-kinase inhibitor & Relaxation & $=$ & Relaxation & 2,3 \\
\hline $\mathrm{CO}$ & Gaseous mediator & Relaxation & $=$ & Relaxation & 5 \\
\hline $\mathrm{H}_{2} \mathrm{~S}$ & Gaseous mediator & Relaxation & $=$ & Relaxation & 5 \\
\hline $17 \mathrm{a}$-estradiol & Estrogen hormone & Relaxation & $=$ & Relaxation & 7 \\
\hline 173 -estradiol & Estrogen hormone & Relaxation & $=$ & Relaxation & 7 \\
\hline
\end{tabular}

Table 8.2 Developmental changes in the response to chicken DA (pulmonary side) to the vasoactive mediators studied in the present thesis

\begin{tabular}{|l|l|l|l|l|l|}
\hline $\begin{array}{l}\text { V a s o a c t i v e } \\
\text { agent }\end{array}$ & $\begin{array}{l}\text { Nature or mechanism } \\
\text { of action }\end{array}$ & 15-d DA & & 19-d DA & Chapter \\
\hline $\mathrm{H}_{2} \mathrm{O}_{2}$ & ROS & No response & $\neq$ & Contraction & 4 \\
\hline BAY K8644 & Calcium-channel activator & No response & $\neq$ & Contraction & 2 \\
\hline Nifedipine & Calcium-channel blocker & Relaxation & $<$ & Relaxation & 2 \\
\hline Hydroxyfasudil & Rho-kinase inhibitor & Relaxation & $<$ & Relaxation & 2,3 \\
\hline Y27632 & Rho-kinase inhibitor & Relaxation & $<$ & Relaxation & 2,3 \\
\hline $\mathrm{H}_{2} \mathrm{~S}$ & Gaseous mediator & Relaxation & $=$ & Relaxation & 5 \\
\hline $\mathrm{CO}$ & Gaseous mediator & Relaxation & $=$ & Relaxation & 5 \\
\hline $15-\mathrm{E}_{2 t}-$ IsoP & Isoprostane & Contraction & $<$ & Contraction & 6 \\
\hline $15-\mathrm{F}_{2 t}-$ IsoP & Isoprostane & Contraction & $<$ & Contraction & 6 \\
\hline
\end{tabular}


The new vasoactive mediators and pathways that are to be studied in the chicken DA model should comply with these two premises to be considered relevant for DA biology: 1) higher response in the PulmDA than in the AoDA and 2) changes with age in the pattern of response.

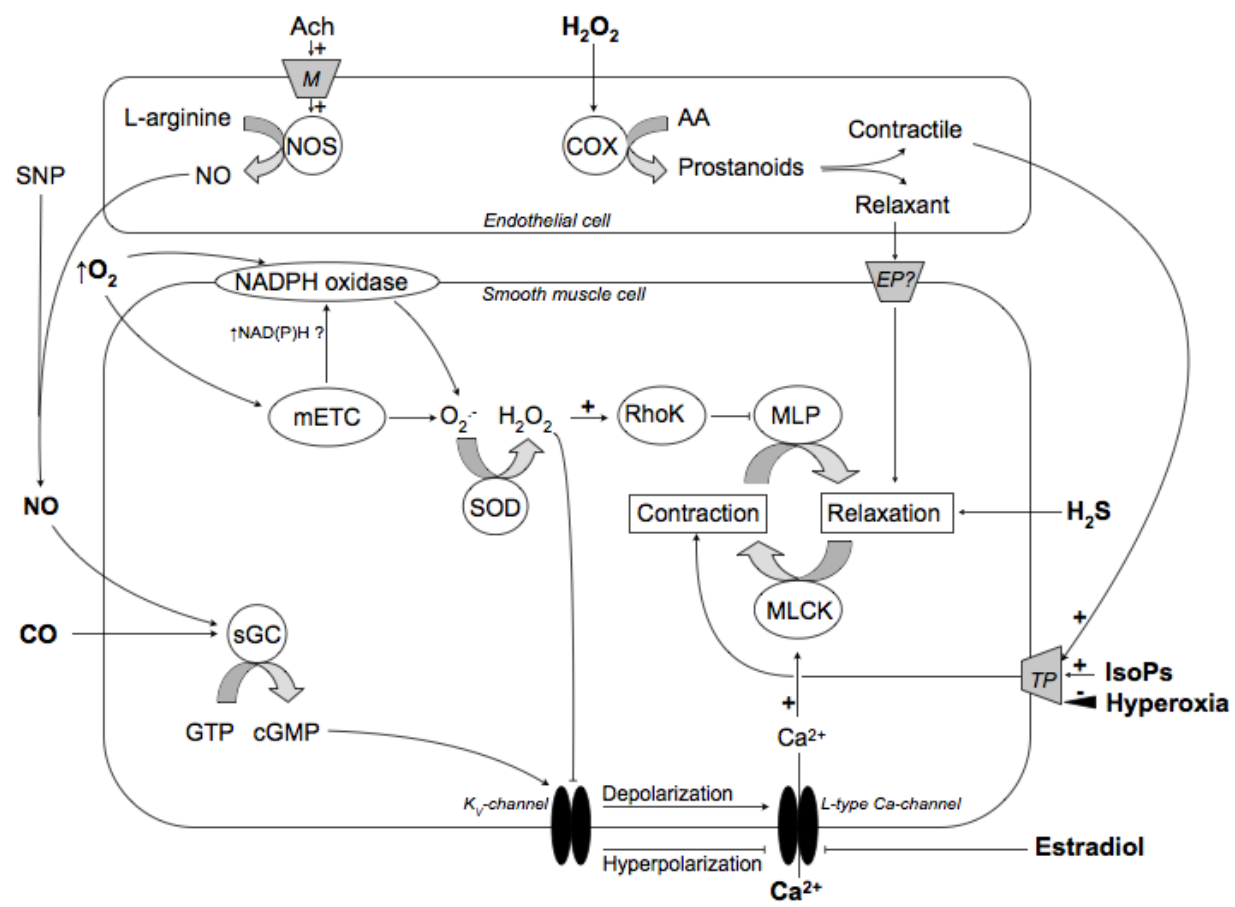

Fig. 8.1 Vasoactive mediators and pathways studied in the present thesis 


\subsection{Oxygen sensing and signaling in the chicken DA}

The specialized homeostatic $\mathrm{O}_{2}$-sensing system to which the DA belongs had already been extensively studied in several mammalian species, including humans (26-44). The most accepted model of $\mathrm{O}_{2}$-induced DA constriction involves a rise in $\mathrm{O}_{2}$ modulating the function of the mitochondrial ETC (the sensor), leading to an increased production of $\mathrm{H}_{2} \mathrm{O}_{2}$ (the mediator) that causes the inhibition of $\mathrm{K}_{v}$ channels (the effector) $(31,32)$, with Rho kinase acting as another downstream effector of the $\mathrm{O}_{2}$-sensing system in the DA $(29,42,43)$. In the chicken embryo we verified the very same pathway (chapter 3 ), proving a conserved mechanism for $\mathrm{O}_{2}$ sensing/signaling in mammalian and nonmammalian DA (Figure 2.5 of chapter 2). Moreover, we demonstrated a developmentally regulated response to $\mathrm{O}_{2}$, which is restricted to the mature PulmDA and involves parallel maturation of the three components: sensor, mediator, and effectors.

A pivotal role for mitochondrial ETC, $\mathrm{H}_{2} \mathrm{O}_{2}, \mathrm{~K}_{\mathrm{v}}$ channels and Rho kinase has been also demonstrated in oxygen signaling in pulmonary and systemic arteries of the chicken embryo $(19,44)$. In addition, the chicken embryo chorioallantoic arteries $(14,21)$, avian homologue of human fetoplacental arteries, also show an exquisite sensitivity to changes in $\mathrm{O}_{2}$ tension. The mechanisms behind hypoxia-induced contraction of chorioallantoic arteries, however, have not yet been elucidated. Nevertheless, the chicken embryo model represents a unique setting in which to investigate the development of vascular oxygen sensing and signaling.

\subsection{Effects of hypoxia and hyperoxia on DA reactivity}

Mammalian fetuses and avian embryos develop in a relatively hypoxic environment and it is under these conditions that the specialized $\mathrm{O}_{2}$-sensitive smooth muscle cells of the DA undergo their specific developmental program in preparation for postnatal closure. The chicken embryo model is highly suited to study the modulatory role of altered prenatal oxygen tension on the developmental trajectory of the $\mathrm{DA}$, as the environmental $\mathrm{O}_{2}$ tension during incubation can be quite easily manipulated. Establishing a similar experimental setup in a mammalian model would confine the mother to a hypoxic/hyperoxic chamber and the fetal organs might also be exposed to maternal-derived circulating factors, released in response to environmental stress (45). 
Changes in $\mathrm{O}_{2}$ tension have profound effects on endothelial and vascular smooth muscle cellular physiology, affecting the transcriptionally regulated expression of vasoactive substances, the modulation of receptor populations, the density and activities of ion channels and the signal transduction pathways involved in modulating vascular tone $(17,46-53)$. Although neither in ovo hypoxia (chapter 3 ) nor hyperoxia (chapter 4) caused significant changes in $\mathrm{O}_{2}$ responsiveness or in the state of patency of the chicken DA, both stimuli induced relevant alterations in the reactivity of the vessel. Specifically, chronic hypoxia during incubation impaired endothelium-dependent and -independent relaxation and altered the responsiveness of DA to adrenergic agonists. On the other hand, hyperoxic incubation impaired the thromboxane prostanoid (TP) receptor-mediated contraction of the chicken DA. Altogether, our data underlie the complexity of the relationship between oxygen homeostasis and DA development.

\subsection{Effects of $\mathrm{H}_{2} \mathrm{~S}$ in the chicken DA}

Oxygen is not the only gaseous mediator with a relevant role in DA reactivity. $\mathrm{CO}_{2}$ and non-respiratory gases, such as $\mathrm{NO}$ and $\mathrm{CO}$, are also ductal vasoactive mediators $(25,54-58)$. In the last years $\mathrm{H}_{2} \mathrm{~S}$ has been identified as an endogenous gaseous transmitter, playing multiple physiological roles in animal systems (59-67). Furthermore, $\mathrm{H}_{2} \mathrm{~S}$ has been proposed as vascular $\mathrm{O}_{2}$ sensor (61). This thesis presents the first study on the vasoactivity of $\mathrm{H}_{2} \mathrm{~S}$ in the DA (chapter 5) (68). In addition, we also analyzed the effects of $\mathrm{CO}$ in the chicken DA. We observed that either $\mathrm{H}_{2} \mathrm{~S}$ or $\mathrm{CO}$ induce relaxation of chicken DA. However, this relaxation is not vessel-specific since PulmDA, AoDA and chicken embryo pulmonary and femoral arteries were similarly relaxed by $\mathrm{H}_{2} \mathrm{~S}$ and $\mathrm{CO}$. Moreover, the presence of precursors/inhibitors of $\mathrm{H}_{2} \mathrm{~S}$ and $\mathrm{CO}$ synthesis did not significantly affect the response of the chicken DA to normoxia/hypoxia and did not affect endothelium-dependent or -independent relaxation. Therefore, our results indicate that the gasotransmitters $\mathrm{H}_{2} \mathrm{~S}$ and $\mathrm{CO}$ are vasoactive in the chicken DA but they do not suggest an important role for endogenous $\mathrm{H}_{2} \mathrm{~S}$ or $\mathrm{CO}$ in the control of chicken ductal reactivity.

Soon after the publication of our study, another group examined the putative role of $\mathrm{H}_{2} \mathrm{~S}$ on the reactivity of mouse DA (69). They found, in this DA, transcripts for the $\mathrm{H}_{2} \mathrm{~S}$ synthetic enzymes cystathionine- $\gamma$-lyase (CSE) and cystathionine- $\beta$-synthase (CBS). The presence of the enzymes was confirmed 
by immunofluorescence microscopy. The isolated mice ductus contracted to inhibitors of CSE (D,L-propargylglycine, PPG) and CBS (amino-oxyacetic acid, $A O A$ ), suggesting that endogenous $\mathrm{H}_{2} S$ is naturally present in the mouse DA and exerts a tonic relaxant action (69). Accordingly, we observed contraction in the chicken DA in response to PPG (68). Unfortunately, Baragatti et al. did not analyze the effect of CSE and CBS inhibition on the response of mouse DA to oxygen or other vasoactive mediators (69). However, they suggest that $\mathrm{H}_{2} \mathrm{~S}$ ductal activity may be favored antenatally by the relatively low-oxygen environment and may abate postnatally with the physiological rise in blood oxygen tension (69). Nevertheless, we did not observe impaired $\mathrm{H}_{2} \mathrm{~S}$-mediated relaxation under postnatal oxygenation conditions (69). Finally, Baragatti et al. suggest that $\mathrm{H}_{2} \mathrm{~S}$ may be the elusive EDHF, at least in the mouse DA (69)). This suggestion is based on the fact that that bradykinin-induced relaxation (in the presence of suppression of $\mathrm{NO}, \mathrm{CO}$ and prostaglandins) is impaired by the presence of either PPG or AOA. Whether $\mathrm{H}_{2} \mathrm{~S}$ is an oxygen sensor or EDHF, elucidating its vasoactive properties in the human DA, is an interesting subject for future research.

\subsection{Response of chicken DA to isoprostanes}

Oxygen is essential to aerobic life but, paradoxically, can be toxic even at atmospheric concentrations. Oxidative stress refers to a relative surplus of reactive oxygen species (ROS) caused by excessive ROS generation and/ or impaired ROS degradation (70-72). Newborns and especially the preterm ones, are more prone to oxidative stress than later in life (73-77)). There are several reasons for this, as these infants very often: (i) are exposed to high oxygen concentrations, (ii) have infections or inflammation, (iii) have reduced antioxidant defense, and (iv) have free iron which is speeding up the Fenton reaction leading to production of ROS. Oxidative stress has been postulated to be implicated in several newborn conditions and in 1988 Saugstad coined the term 'oxygen radical disease of neonatology' (78). The idea was that oxidative stress may affect different organs, often simultaneously, giving rise to different signs according to the organ most affected. Saugstad included bronchopulmonary dysplasia/chronic lung disease, retinopathy of prematurity and necrotizing enterocolitis in this entity. Later it became clear that free radicals are involved in perinatal asphyxia and periventricular leukomalacia as well as in regulating the systemic and pulmonary circulation and the DA (73). If the concept of 'oxygen radical disease in neonatology' is correct it means that these 
conditions mentioned are not different diseases, but belong to the same entity giving rise to different symptoms according to which organs are most affected. However, one important consideration is that ROS are not always associated with deleterious effects and under physiological conditions they appear to also have essential functions, for example, within the innate immune response, cellular signaling, control of cellular proliferation and differentiation, oxygen sensing, vascular tone, and angiogenesis. Consequently, interfering with ROS in a too broad and unspecific manner may affect its beneficial roles and can result in pathologies as well, a phenomenon termed reductive stress (70).

Isoprostanes (IsoPs) are a unique series of prostaglandin-like compounds formed in vivo from the free radical-catalyzed peroxidation of arachidonic acid independent of the cyclooxygenase (COX) enzymes. (79-80). Initially, IsoPs were recognized as valuable markers of oxidative stress and numerous pathological conditions have been shown to be associated with increases in urinary, plasma, and tissue levels of IsoPs. Later it became apparent that IsoPs exhibit significant bioactivity and could contribute to the functional consequences of oxidative stress and participate in oxygen signaling (81-82)), particularly during the transition to extra-uterine life (83-86I)). Surprisingly, until our study (chapter 6) (87)), the possible vascular effects of IsoPs in the DA had not been investigated. We observed that $15-E_{2 t}$-IsoP evoked a potent and efficacious, TP receptor-mediated, contraction in the chicken DA. On the other hand, $15-F_{2 t}-I s o P$ is probably acting as a partial agonist at TP receptors. The contractile response of the PulmDA to IsoPs increased with development and both $15-E_{2 t}$ IsoP and $15-F_{2 t}$-IsoP were particularly efficacious in contracting the PulmDA in comparison with the AoDA and the femoral or the pulmonary arteries. Altogether, our data suggest that IsoPs, acting through TP receptors, may play a role in the control of chicken DA tone and could participate in its closure. In agreement with our results, Chen et al reported that $15-E_{2 t}-I s o P$ and $15-\mathrm{F}_{2 \mathrm{t}}$-IsoP contracted the mouse DA through activation of TP receptors (88). Interestingly, exposure of the preterm DA to $15-\mathrm{E}_{2 t}$-IsoP caused unexpected DA relaxation that was reversed by $\mathrm{EP}_{4}$ antagonism. Further investigation revealed the predominance of the TP receptor in the DA of term mice, whereas the $\mathrm{EP}_{4}$ receptor was expressed and functionally active from mid-gestation onward (88).

The majority of the studies analyzing the effect of prostanoids in the DA have focused on the COX-PGE $E_{2}-E P$ receptor signaling pathway. This pathway plays a critical role in regulation of DA maturation and closure after birth. However, recent investigations, including our studies, on the vasoactivity of $\mathrm{TXA}_{2}$ and 
IsoPs in the DA have underlined the relevant contribution of TP receptors to ductal (patho)biology. $\mathrm{EP}_{4}$ and TP are the predominant prostanoid receptors in the DA of mice, humans, and other species $(58,89,90)$. It has been suggested that with increasing maturity, the balance between $\mathrm{EP}_{4}$ and TP shifted in favor of the contractile effects of TP stimulation (88). Moreover, Loftin et al. showed that TP agonists can forcefully close the PDA of COX-deficient mice at term gestation (91). TP receptor stimulation promotes contraction and neointima formation in the rat DA $(92,93)$. However, it should be noted that the PDA phenotype has not been reported in TP knockout mice (94) and that a TP antagonist itself does not inhibit rat DA closure after birth ((null) (null)). Accordingly, we observed that the presence of a TP receptor antagonist did not affect $\mathrm{O}_{2}$-induced contraction in the chicken DA (unpublished observation). Therefore, it is unlikely that intrinsic TXA 2 -TP signaling plays a pivotal role in spontaneous DA closure.

\subsection{Lack of sexual dimorphism in chicken DA reactivity}

The "male disadvantage" is a widespread recognition that, compared to females, human male neonates are at higher risk for prematurity, as well as pulmonary, neurological, gastrointestinal and cardiovascular prematurity-related conditions (95-97). The contributing biological mechanisms remain poorly understood, but are likely to be multifactorial. It has been proposed that the male disadvantage begins in utero, when gonadal steroid production already differs strongly by sex (96). Sex hormones have an important influence on cardiovascular (patho)physiology (98), yet we were the first to investigate the presence of sex differences in vascular reactivity and the vascular effects of sex hormones in the DA (chapter 7). We did not observe significant sex-related differences in chicken DA response to oxygen and other contractile or relaxant stimuli, suggesting that at least the chicken DA oxygen sensitivity and contractile system are equally developed in males and females. In addition, we demonstrated that the estrogen $17 \beta$-estradiol induced endothelium-independent relaxation of chicken DA. This relaxation is not mediated by estrogen receptor activation and is, at least partially, due to inhibition of $\mathrm{Ca}^{2+}$ entry from extracellular space. Nevertheless, $17 \beta$-estradiol-induced relaxation was similar in the two parts of the chicken DA (i.e., PulmDA and AoDA), indicating a lack of specificity for the DA. 


\subsection{Future perspectives}

As stated in the introduction, the hope for future targeted therapies and for the prevention of PDA requires knowledge of the fundamental mechanisms controlling its development and pathogenesis $(57,58)$. In line with the present thesis, an important part of future research lies in testing new hypotheses on DA (patho)biology, including -but not limited to- the following:

8.7.1. Role of and NADPH-oxidase and ceramide in DA oxygen sensing and signaling

The DA behaves exactly opposite to the resistance pulmonary arteries (PAs), in that it contracts during normoxia and relaxes in hypoxia. However, normoxic contraction of the DA appears to involve the same mechanisms as hypoxic pulmonary vasoconstriction (HPV) (99). Consequently, an important part of the hypotheses on the response of the DA to oxygen arise from previous knowledge on the mechanisms of HPV. NADPH-oxidase and ceramide have emerged as potential participants in the signaling cascade of HPV (44, 100-102) and, therefore, the possible role of these mediators in normoxic contraction of the DA warranted further elucidation:

8.7.1.1 NADPH oxidases are multiprotein complexes. Seven isoforms of their catalytic subunits exist, annotated as NOX1-5 and DUOX1-2 (recently also termed as NOX6-7).These NOX catalytic subunits represent the only known enzyme family with the sole function to produce ROS $(103,104)$. Multiple NOX subtypes, simultaneously present in different subcellular compartments, produce specific amounts of superoxide, some of which is rapidly converted to hydrogen peroxide. The identity and location of these ROS, and of the enzymes that degrade them, determine their downstream signaling pathways (105). As mentioned above, NOX and mitochondria have been proposed to be major sources of intracellular ROS generation mediating hypoxic responses in PA smooth muscle cells $(99,105,106)$. Thus, recent experimental findings suggest that NOX4 is the source of $\mathrm{H}_{2} \mathrm{O}_{2}$ controlling the HPV response $(105,107,108)$. Hypoxia can also induce an increase in the availability of the NOX substrate NADPH in PAs potentially through activating glucose-6-phosphate dehydrogenase (105). In contrast to HPV, the role of NOX in the normoxic contraction of the DA has been scarcely investigated. Interestingly, incubation with the NOX inhibitor VAS2870 impaired normoxic contraction of chicken DA as well as hypoxic contraction of the PA (44). In the near future, we intend to 
investigate the potential role of NOX as oxygen sensor in the DA. In order to achieve this aim, we would analyze the effects of selective NOX inhibitors (104, $109)$ in the DA response to normoxia as well as oxygen-induced contraction in transgenic and knockout mice for the NOX enzymes $(110,111)$.

8.7.1.2 Ceramide is synthesized from membrane sphingomyelin by sphingomyelinases (SMase) which are activated by multiple membrane receptors and non-receptor stimuli (112). Neutral SMase (nSMase)-derived ceramide acts as a critical mediator in the HPV response in rats by increasing ROS production via NOX $(101,102)$. Recently, using the chicken model, we observed that inhibition of the ceramide-generating enzyme nSMase reduced the hypoxic vasoconstriction in PA and the normoxic contraction of the DA. Moreover, ceramide content and $\mathrm{H}_{2} \mathrm{O}_{2}$ production were increased by hypoxia in PAs and by normoxia in the DA (44). Accordingly, ceramide mimicked the contractile responses of hypoxia in PAs and those of normoxia in the DA. In addition, ceramide inhibited $\mathrm{Kv}$ currents in PA and DA smooth muscle cells. Finally, the role of nSMase in acute oxygen sensing was confirmed in human PA and DA (44). However, the mechanisms matching the putative $\mathrm{O}_{2}$ sensor(s) (i.e., mitochondria and/or NOX) and ceramide remain unknown. Intriguingly, nSMase is a redox-sensitive enzyme that can be activated by an increase in ROS (12, 113). Thus, one possibility is that in both situations, hypoxia in PA and normoxia in DA, nSMase are activated by mitochondria or NOX-derived ROS. It is conceivable that normoxia increases mitochondrial ROS generation in the DA by providing more oxygen as substrate. Likewise, hypoxia is able to increase mitochondrial-derived ROS in PA (and probably in systemic arteries) (114). Therefore, activation of nSMase, production of ceramide and subsequent NOXderived ROS might represent an amplification pathway downstream the mitochondrial sensor $(100-102,115)$ present only in specialized oxygen sensing cells. This hypothesis is under current scrutiny in our laboratory.

\subsubsection{Mitochondrial biogenesis and dynamics during the maturation of the $D A$}

Mitochondrial biogenesis results from the coordinated expression of nuclear and mitochondrial genomes and involves both proliferation and differentiation processes, which imply an increase in mitochondrial content and maturation of the functional capabilities of pre-existing mitochondria (116). Mitochondrial biogenesis starts the mitochondrial cycle by division of pre-existing organelles, and mitophagy ends it by degradation of impaired mitochondria. In between, mitochondria undergo several cycles of fission and fusion that generate multiple 
heterogeneous mitochondria or interconnected mitochondrial networks depending on the physiological conditions (116).

Mitochondrial biogenesis and dynamics during fetal development has been studied in several organs such as the brain, lung, liver, kidney or heart (117-119). However, the basic parameters of bioenergetics and mitochondrial structure and function in the DA are still largely unexplored. Very recently, Hong et al., reported that physiological increases in $\mathrm{PO}_{2}$ rapidly induces mitochondrial fission in DA smooth muscle by stimulating phosphorylation of the GTPase dynamin-related protein 1 (Drp1). Fission subsequently increases activity of Complex 1 in the mitochondrial electron transport chain and Drp1 inhibition selectively blocked oxygen-mediated DA constriction and, if sustained, prevented DA closure in a tissue culture model (120). This suggests that structural changes in mitochondria are a key early step in $\mathrm{O}_{2}$ sensing (120). Therefore, a reduced capacity of the mitochondria to undergo the process of fission as well as other functional or structural alterations may well be involved in the immature response of the preterm DA to $\mathrm{O}_{2}$. It would be very interesting to characterize the developmental changes in mitochondrial number and ultrastructure in the chicken DA, as well as analyzing developmental changes in mitochondrial function and expression of genes involved in the organelle biogenesis and function.

Prenatal glucocorticoids are standard of care for women at high risk of preterm delivery prior to 34 weeks' gestation because randomized, controlled trials and extensive meta-analyses demonstrate decreased mortality and improved outcomes, primarily related to less respiratory distress syndrome and a decreased incidence of intraventricular hemorrhages (121). Several reports suggest that mitochondrial maturation may be controlled by steroid hormones $(117,119)$. Therefore, analyzing the effects of prenatal corticosteroids in DA mitochondrial biogenesis and dynamics, as well as in human umbilical and fetoplacental blood vessels could provide us with interesting new insights into developmental vascular biology.

\subsubsection{Role of platelets in DA closure}

Very recently, Echtler et al. proposed a model of closure of mice DA in which the initial constriction results in changes in the DA wall which adopts a prothrombotic phenotype with endothelial activation, deposition of von Willebrand factor and fibrin(ogen), and eventually endothelial cell detachment 
from the internal elastic lamina, leading to collagen exposure (122). This process triggers the accumulation of platelets circulating in the residual DA lumen, leading to the formation of a platelet plug that seals the lumen. In preterm human infants, like in mice, the DA is a much thinner vessel, able to extract all of the oxygen it needs from blood flow within its lumen. Therefore, and even when it does constrict, the premature DA frequently fails to develop profound hypoxia and neointimal expansion is markedly diminished (123). The underdeveloped neointimal mounds fail to occlude the residual lumen and, as a result, the DA remains patent. Future research should address the question of whether platelet aggregation and thrombus formation can substitute for the lack of neointimal mounds in preterm human infants (123). Echtler et al also performed a retrospective, multivariate analysis in a cohort of 123 infants born at 24 to 30 weeks of gestation. They reported that a platelet count between 101 and 140 on the first day of life was an independent predictor of PDA (122). Since the publication of the above study, several additional cohorts with discordant results have been reported (124-130). A very recent meta-analysis, which combines data from 11 study cohorts (3479 preterm infants), shows that a low platelet count in the first day(s) of life is a modest, but significant, risk factor for PDA (131). However, the low robustness of the association and the high degree of statistical heterogeneity among studies suggest that the link between platelet counts and DA closure might not be clinically relevant (131).

Recently, we approached the question of the relationship between DA closure and platelets from a clinical and experimental perspective. Thus, we are analyzing the role of platelet number in association with the genetic variants linked to platelet hypo- and hyperresponsive phenotypes (132) as PDA risk factors in a cohort of very pretern infants. Our experimental approach to the role of platelets in DA closure is based on the hypothesis that platelet-released vasoactive agonists are substantial contributors to DA closure. Activated platelets release different substances with vasoactive properties, like serotonin, thromboxane $A_{2}\left(T X A_{2}\right)$, platelet activating factor (PAF) or thrombin. The three former agonists are vasoactive in the DA (55). Recently, we started a research project aimed at determining the possible vasoactive effects of thrombin in the DA. Cellular effects of thrombin are mediated by protease activated receptors (PARs), members of the G protein-coupled receptors that carry their own ligand, which remains cryptic until unmasked by proteolytic cleavage. PAR-1 and PAR-2 have been shown to be involved in regulating vascular tone $(133,134)$. Thrombin activates PAR-1, whereas trypsin activates PAR-1 and PAR-2. Our preliminary results indicate that thrombin, acting through PAR-1, is a selective vasoconstrictor in the DA of the chicken, providing the first functional evidence 
for a role of PARs in DA biology. Currently, we are analyzing the expression of PARs in the DA of several species (human. lamb, rat, mouse and chicken). In addition it would be of great interest to investigate whether PAR-1 knockout mice (135) present delayed closure of the DA.

\subsubsection{Paracetamol for the treatment of PDA}

Standard pharmacologic treatment of PDA involves the administration of the PG synthetase inhibitors indomethacin and/or ibuprofen. Several reports have been published concerning an association between paracetamol exposure and PDA closure within the past few years suggesting that this might be an alternative for PDA treatment (136-139). However, there are limited data regarding the pharmacokinetics, pharmacodynamics and toxicity of paracetamol in preterm neonates (140), Several randomized controlled are currently conducted comparing paracetamol with ibuprofen or indomethacin for the efficacy of closing a PDA. Very recently, Ohlsson \& Shah concluded in a meta-analysis that, despite the limited number of infants studied, oral paracetamol appears to be as effective in closing a PDA as oral ibuprofen (141). However, they also express their concerns about the possible side effects of paracetamol in the developing brain..(141). Nevertheless, the pharmacological mechanisms behind the efficacy of paracetamol, when ibuprofen and/or indomethacin have failed, remain unknown. The enzyme PG synthetase has two two components, a cyclooxygenase $(\mathrm{COX})$ and a peroxidase that operate at distinct sites and with different catalytic activities. At the active COX site, arachidonic acid undergoes oxygenation and forms $P G_{2}$, which is then acted on by the peroxidase component of the enzyme, forming $\mathrm{PGH}_{2}$. Indomethacin and ibuprofen compete with arachidonic acid for the active COX site (142). Thus, the potency of these drugs is influenced by endogenous arachidonic acid levels. Paracetamol also inhibits PG synthetase activity but its precise mechanism of action remains controversial. Paracetamol seems to act at the peroxidase segment of the enzyme (142). Peroxidase is activated at 10 -fold-lower peroxide concentrations than is COX. Therefore, paracetamol-mediated PG synthetase inhibition is facilitated at reduced local peroxide concentrations (e.g., hypoxia). Theoretically, these differences would permit peroxidase inhibition to be optimally effective under conditions in which COX inhibition is less active (136). Very recently ElKhuffash et al demonstrated that paracetamol is less potent than indomethacin for constriction of the mouse DA ex vivo (143). Currently, we are evaluating the comparative effects of paracetamol, indomethacin and ibuprofen in the DA of chickens, rats and rabbits under normoxic and hypoxic conditions. The inhibitory 
effects of the drugs on PG synthesis will be also evaluated in human umbilical vessels.

\subsubsection{Effects of commonly used medications on DA closure}

New drugs or even those that are commonly used in the neonatal intensive care unit may have unexpected effects on the ability of the DA to spontaneously close, resulting in increased risks for PDA (144). Data demonstrating relaxation of the DA by gentamicin and other aminoglycoside antibiotics, by cimetidine and other $\mathrm{H}_{2}$ receptor antagonists, and by heparin are provided as examples of neonatal therapies that may induce DA relaxation (144). The chicken DA is a feasible model to test the putative ductal effects of drugs or therapeutic strategies to which the infants are exposed in utero or after birth. In this sense, we recently analyzed the effects in the DA of hypecarbia (8)) and magnesium sulfate $\left(\mathrm{MgSO}_{4}\right)$ (145). Permissive hypercarbia in which higher levels of $\mathrm{PaCO}_{2}$ are tolerated is often used in the ventilatory management of extremely premature infants and $\mathrm{MgSO}_{4}$ is widely used as tocolytic agent and for the prophylaxis of seizures in pre-eclampsia. Antenatal exposure to $\mathrm{MgSO}_{4}$ has been associated with a higher risk of PDA in preterm infants. We observed that either hypercarbia or $\mathrm{MgSO}_{4}$, at clinically relevant concentrations, evoked relaxation in the chicken DA. Currently, we are also evaluating the effects of hypothermia on DA reactivity. Mild systemic hypothermia has been shown to reduce the combined chance of death or disability in asphyxiated term newborns and has become the standard of care for neonatal asphyxia (146). However, hypothermia can be accompanied by increased systemic, as well as pulmonary vascular resistance, and decreased heart rate resulting in decreased cardiac output $(147,148)$ Cerebral perfusion is related directly to cardiac output in the absence of major shunts across the foramen ovale or DA. Thus, a delayed closure of the DA during hypothermia may reduce cerebral perfusion. Other drugs used in the neonate and with possible effects on DA reactivity and closure are phosphodiesterase (PDE) inhibitors, such as milrinone and amrinone (PDE3 inhibitors) or sildenafil (PDE5 inhibitor), diuretics, such as furosemide, and respiratory stimulants, such as caffeine (144). 


\section{References}

1. Wartofsky MW. Models: Representation and the Scientific Understanding. Dordrecht: D. Reidel Publishing Company; 1979.

2. Festing MF. Design and statistical methods in studies using animal models of development. ILAR J. 2006;47(1):5-14.

3. Brown WR, Hubbard SJ, Tickle C, Wilson SA. The chicken as a model for largescale analysis of vertebrate gene function. Nat Rev Genet. 2003 Feb;4(2):87-98.

4. Agren P, Cogolludo AL, Kessels CG, Perez-Vizcaino F, De Mey JG, Blanco CE, et al. Ontogeny of chicken ductus arteriosus response to oxygen and vasoconstrictors. Am J Physiol Regul Integr Comp Physiol. 2007 Jan;292(1):R485-96.

5. Agren $\mathrm{P}$, van der Sterren $\mathrm{S}$, Cogolludo AL, Blanco CE, Villamor E. Developmental changes in the effects of prostaglandin E2 in the chicken ductus arteriosus. J Comp Physiol B. 2009 Feb;179(2):133-43.

6. Agren P, van der Sterren S, Cogolludo AL, Frazziano G, de Mey JG, Blanco CE, et al. Developmental changes in endothelium-dependent relaxation of the chicken ductus arteriosus. J Physiol Pharmacol. 2008 Mar;59(1):55-76.

7. Moonen RM, Agren P, Cogolludo AL, Perez Vizcaino F, Villamor E. Response of chicken ductus arteriosus to hypercarbic and normocarbic acidosis. Neonatology. Jun; 98(1):47-56.

8. Schuurman MJ, Villamor E. Endothelium-dependent contraction induced by acetylcholine in the chicken ductus arteriosus involves cyclooxygenase-1 activation and TP receptor stimulation. Comp Biochem Physiol A Mol Integr Physiol. Sep;157(1):28-34.

9. Belanger C, Copeland J, Muirhead D, Heinz D, Dzialowski EM. Morphological changes in the chicken ductus arteriosi during closure at hatching. Anat Rec (Hoboken). 2008 Aug;291(8):1007-15.

10. Copeland J, Dzialowski EM. Effects of hypoxic and hyperoxic incubation on the reactivity of the chicken embryo (Gallus gallus) ductus arteriosi in response to catecholamines and oxygen. Exp Physiol. 2009 Jan;94(1):152-61.

11. Greyner H, Dzialowski EM. Mechanisms mediating the oxygen-induced vasoreactivity of the ductus arteriosus in the chicken embryo. Am J Physiol Regul Integr Comp Physiol. 2008 Nov;295(5):R1647-59.

12. Sutendra G, Michelakis ED. The chicken embryo as a model for ductus arteriosus developmental biology: cracking into new territory. Am J Physiol Regul Integr Comp Physiol. 2007 Jan;292(1):R481-4.

13. Carlo WF, Villamor E, Ambalavanan N, DeMey JG, Blanco CE. Chronic exposure to cigarette smoke extract impairs endothelium-dependent relaxation of chicken embryo pulmonary arteries. Biol Neonate. 2001;80(3):247-50.

14. Lindgren I, Zoer B, Altimiras J, Villamor E. Reactivity of chicken chorioallantoic arteries, avian homologue of human fetoplacental arteries. J Physiol Pharmacol. 2010 Oct;61(5):619-28. 
15. Moonen RM, Kessels CG, Zimmermann LJ, Villamor E. Mesenteric artery reactivity and small intestine morphology in a chicken model of hypoxia-induced fetal growth restriction. J Physiol Pharmacol. 2012 Dec;63(6):601-12.

16. Ruijtenbeek K, Kessels CG, Villamor E, Blanco CE, De Mey JG. Direct effects of acute hypoxia on the reactivity of peripheral arteries of the chicken embryo. Am $\mathrm{J}$ Physiol Regul Integr Comp Physiol. 2002 Aug;283(2):R331-8.

17. Villamor E, Kessels CG, Ruijtenbeek K, van Suylen RJ, Belik J, de Mey JG, et al. Chronic in ovo hypoxia decreases pulmonary arterial contractile reactivity and induces biventricular cardiac enlargement in the chicken embryo. Am J Physiol Regul Integr Comp Physiol. 2004 Sep;287(3):R642-51.

18. Villamor E, Ruijtenbeek K, Pulgar V, De Mey JG, Blanco CE. Vascular reactivity in intrapulmonary arteries of chicken embryos during transition to ex ovo life. Am J Physiol Regul Integr Comp Physiol. 2002 Mar;282(3):R917-27.

19. Zoer B, Cogolludo AL, Perez-Vizcaino F, De Mey JG, Blanco CE, Villamor E. Hypoxia sensing in the fetal chicken femoral artery is mediated by the mitochondrial electron transport chain. Am J Physiol Regul Integr Comp Physiol. 2010 Apr; 298(4):R1026-34.

20. Zoer B, Kessels L, Vereijken A, De Mey JG, Bruggeman V, Decuypere E, et al. Effects of prenatal hypoxia on pulmonary vascular reactivity in chickens prone to pulmonary hypertension. J Physiol Pharmacol. 2009 Mar;60(1):119-30.

21. Lindgren I, Crossley D, 2nd, Villamor E, Altimiras J. Hypotension in the chronically hypoxic chicken embryo is related to the beta-adrenergic response of chorioallantoic and femoral arteries and not to bradycardia. Am J Physiol Regul Integr Comp Physiol. 2011 Oct;301(4):R1161-8.

22. Bergwerff M, Verberne ME, DeRuiter MC, Poelmann RE, Gittenberger-de Groot AC. Neural crest cell contribution to the developing circulatory system: implications for vascular morphology? Circ Res. 1998 Feb 9;82(2):221-31.

23. Bergwerff M, DeRuiter MC, Gittenberger-de Groot AC. Comparative anatomy and ontogeny of the ductus arteriosus, a vascular outsider. Anat Embryol (Berl). 1999 Dec;200(6):559-71.

24. Cogolludo AL, Moral-Sanz J, van der Sterren S, Frazziano G, van Cleef AN, Menendez $\mathrm{C}$, et al. Maturation of $\mathrm{O} 2$ sensing and signaling in the chicken ductus arteriosus. Am J Physiol Lung Cell Mol Physiol. 2009 Oct;297(4):L619-30.

25. Moonen RM, Agren P, Cogolludo AL, Perez Vizcaino F, Villamor E. Response of Chicken Ductus Arteriosus to Hypercarbic and Normocarbic Acidosis. Neonatology. 2009 Dec 10;98(1):47-56.

26. Archer SL, Souil E, Dinh-Xuan AT, Schremmer B, Mercier JC, EI Yaagoubi A, et al. Molecular identification of the role of voltage-gated $\mathrm{K}+$ channels, Kv1.5 and Kv2.1, in hypoxic pulmonary vasoconstriction and control of resting membrane potential in rat pulmonary artery myocytes. J Clin Invest. 1998 Jun 1;101(11):2319-30.

27. Archer SL, Wu XC, Thebaud B, Nsair A, Bonnet S, Tyrrell B, et al. Preferential expression and function of voltage-gated, O2-sensitive $\mathrm{K}+$ channels in resistance 
pulmonary arteries explains regional heterogeneity in hypoxic pulmonary vasoconstriction: ionic diversity in smooth muscle cells. Circ Res. 2004 Aug 6;95(3): 308-18.

28. Hampl V, Bibova J, Stranak Z, Wu X, Michelakis ED, Hashimoto K, et al. Hypoxic fetoplacental vasoconstriction in humans is mediated by potassium channel inhibition. Am J Physiol Heart Circ Physiol. 2002 Dec;283(6):H2440-9.

29. Kajimoto H, Hashimoto K, Bonnet SN, Haromy A, Harry G, Moudgil R, et al. Oxygen activates the Rho/Rho-kinase pathway and induces RhoB and ROCK-1 expression in human and rabbit ductus arteriosus by increasing mitochondria-derived reactive oxygen species: a newly recognized mechanism for sustaining ductal constriction. Circulation. 2007 Apr 3;115(13):1777-88.

30. Michelakis E, Rebeyka I, Bateson J, Olley P, Puttagunta L, Archer S. Voltagegated potassium channels in human ductus arteriosus. Lancet. 2000 Jul 8;356(9224): 134-7.

31. Michelakis ED, Hampl V, Nsair A, Wu X, Harry G, Haromy A, et al. Diversity in mitochondrial function explains differences in vascular oxygen sensing. Circ Res. 2002 Jun 28;90(12):1307-15.

32. Michelakis ED, Rebeyka I, Wu X, Nsair A, Thebaud B, Hashimoto K, et al. O2 sensing in the human ductus arteriosus: regulation of voltage-gated $\mathrm{K}+$ channels in smooth muscle cells by a mitochondrial redox sensor. Circ Res. 2002 Sep 20;91(6): 478-86.

33. Michelakis ED, Thebaud B, Weir EK, Archer SL. Hypoxic pulmonary vasoconstriction: redox regulation of $\mathrm{O} 2$-sensitive $\mathrm{K}+$ channels by a mitochondrial $\mathrm{O} 2$ sensor in resistance artery smooth muscle cells. J Mol Cell Cardiol. 2004 Dec;37(6): 1119-36.

34. Moudgil R, Michelakis ED, Archer SL. Hypoxic pulmonary vasoconstriction. J Appl Physiol. 2005 Jan;98(1):390-403.

35. Reeve HL, Tolarova S, Nelson DP, Archer S, Weir EK. Redox control of oxygen sensing in the rabbit ductus arteriosus. J Physiol. 2001 May 15;533(Pt 1):253-61.

36. Reeve HL, Weir EK, Archer SL, Cornfield DN. A maturational shift in pulmonary K+ channels, from Ca2+ sensitive to voltage dependent. Am J Physiol. 1998 Dec;275(6 Pt 1):L1019-25.

37. Thebaud B, Michelakis ED, Wu XC, Moudgil R, Kuzyk M, Dyck JR, et al. Oxygen-sensitive $\mathrm{Kv}$ channel gene transfer confers oxygen responsiveness to preterm rabbit and remodeled human ductus arteriosus: implications for infants with patent ductus arteriosus. Circulation. 2004 Sep 14;110(11):1372-9.

38. Thebaud B, Wu XC, Kajimoto H, Bonnet S, Hashimoto K, Michelakis ED, et al. Developmental absence of the $\mathrm{O} 2$ sensitivity of L-type calcium channels in preterm ductus arteriosus smooth muscle cells impairs $\mathrm{O} 2$ constriction contributing to patent ductus arteriosus. Pediatr Res. 2008 Feb;63(2):176-81. 
39. Tristani-Firouzi M, Reeve HL, Tolarova S, Weir EK, Archer SL. Oxygen-induced constriction of rabbit ductus arteriosus occurs via inhibition of a 4-aminopyridine-, voltage-sensitive potassium channel. J Clin Invest. 1996 Nov 1;98(9):1959-65.

40. Weir E, Lopez-Barneo J, Buckler K, SL A. Acute oxygen-sensing mechanisms. New Engl J Med. 2005;353(19):2042-55.

41. Weir EK, Archer SL. The role of redox changes in oxygen sensing. Respir Physiol Neurobiol. Dec 31;174(3):182-91.

42. Clyman RI, Waleh N, Kajino H, Roman C, Mauray F. Calcium-dependent and calcium-sensitizing pathways in the mature and immature ductus arteriosus. Am $\mathrm{J}$ Physiol Regul Integr Comp Physiol. 2007 Oct;293(4):R1650-6.

43. Hong Z, Hong F, Olschewski A, Cabrera JA, Varghese A, Nelson DP, et al. Role of store-operated calcium channels and calcium sensitization in normoxic contraction of the ductus arteriosus. Circulation. 2006 Sep 26;114(13):1372-9.

44. Moreno L, Moral-Sanz J, Morales-Cano D, Barreira B, Moreno E, Ferrarini A, et al. Ceramide Mediates Acute Oxygen Sensing in Vascular Tissues. Antioxid Redox Signal. 2013 Aug 21.

45. Ruijtenbeek K, De Mey JG, Blanco CE. The chicken embryo in developmental physiology of the cardiovascular system: a traditional model with new possibilities. Am J Physiol Regul Integr Comp Physiol. 2002 Aug;283(2):R549-50; author reply R50-1.

46. Ruijtenbeek K, Kessels LC, De Mey JG, Blanco CE. Chronic moderate hypoxia and protein malnutrition both induce growth retardation, but have distinct effects on arterial endothelium-dependent reactivity in the chicken embryo. Pediatr Res. 2003 Apr; 53(4):573-9.

47. Marino M, Beny JL, Peyter AC, Bychkov R, Diaceri G, Tolsa JF. Perinatal hypoxia triggers alterations in $\mathrm{K}+$ channels of adult pulmonary artery smooth muscle cells. Am J Physiol. 2007 Nov;293(5):L1171-82.

48. Li D, Laubach VE, Johns RA. Upregulation of lung soluble guanylate cyclase during chronic hypoxia is prevented by deletion of eNOS. Am J Physiol. 2001 Aug; 281(2):L369-76.

49. McNamara PJ, Murthy P, Kantores C, Teixeira L, Engelberts D, van Vliet T, et al. Acute vasodilator effects of Rho-kinase inhibitors in neonatal rats with pulmonary hypertension unresponsive to nitric oxide. Am J Physiol. 2008 Feb;294(2):L205-13.

50. Herrera EA, Pulgar VM, Riquelme RA, Sanhueza EM, Reyes RV, Ebensperger $G$, et al. High-altitude chronic hypoxia during gestation and after birth modifies cardiovascular responses in newborn sheep. Am J Physiol Regul Integr Comp Physiol. 2007 Jun;292(6):R2234-40.

51. Ruijtenbeek K, le Noble FA, Janssen GM, Kessels CG, Fazzi GE, Blanco CE, et al. Chronic hypoxia stimulates periarterial sympathetic nerve development in chicken embryo. Circulation. 2000 Dec 5;102(23):2892-7.

52. Newman JH, Loyd JE, English DK, Ogletree ML, Fulkerson WJ, Brigham KL. Effects of $100 \%$ oxygen on lung vascular function in awake sheep. J Appl Physiol. 1983 May;54(5):1379-86. 
53. Belik J, Jankov RP, Pan J, Tanswell AK. Chronic O2 exposure enhances vascular and airway smooth muscle contraction in the newborn but not adult rat. $\mathrm{J} \mathrm{Appl}$ Physiol. 2003 Jun;94(6):2303-12.

54. Baragatti B, Brizzi F, Barogi S, Laubach VE, Sodini D, Shesely EG, et al. Interactions between NO, CO and an endothelium-derived hyperpolarizing factor (EDHF) in maintaining patency of the ductus arteriosus in the mouse. Br J Pharmacol. 2007 May; 151(1):54-62.

55. Smith GC. The pharmacology of the ductus arteriosus. Pharmacol Rev. 1998 Mar;50(1):35-58.

56. Coceani F, Kelsey L. Endothelin-1 release from the lamb ductus arteriosus: are carbon monoxide and nitric oxide regulatory agents? Life Sci. 2000 May 19;66(26): 2613-23.

57. Stoller JZ, DeMauro SB, Dagle JM, Reese J. Current Perspectives on Pathobiology of the Ductus Arteriosus. J Clin Exp Cardiolog. 2012;S8:001.

58. Bokenkamp R, DeRuiter MC, van Munsteren C, Gittenberger-de Groot AC. Insights into the pathogenesis and genetic background of patency of the ductus arteriosus. Neonatology. 2010 Jun;98(1):6-17.

59. Meng G, Ma Y, Xie L, Ferro A, Ji Y. Emerging role of hydrogen sulfide in hypertension and related cardiovascular diseases. Br J Pharmacol. 2014 Sep 10.

60. Leffler $\mathrm{CW}$, Parfenova $\mathrm{H}$, Jaggar JH, Wang R. Carbon monoxide and hydrogen sulfide: gaseous messengers in cerebrovascular circulation. J Appl Physiol. 2006 Mar; 100(3):1065-76.

61. Olson KR. Hydrogen sulfide and oxygen sensing: implications in cardiorespiratory control. J Exp Biol. 2008;211(Pt 17):2727-34.

62. Zhao W, Wang R. H(2)S-induced vasorelaxation and underlying cellular and molecular mechanisms. Am J Physiol-Heart C. 2002;283(2):H474-80.

63. Zhao W, Zhang J, Lu Y, Wang R. The vasorelaxant effect of $\mathrm{H}(2) \mathrm{S}$ as a novel endogenous gaseous K(ATP) channel opener. EMBO J. 2001;20(21):6008-16.

64. Lowicka E, Beltowski J. Hydrogen sulfide (H2S) - the third gas of interest for pharmacologists. Pharmacol Rep. 2007;59(1):4-24.

65. Lim JJ, Liu YH, Khin ES, Bian JS. Vasoconstrictive effect of hydrogen sulfide involves downregulation of cAMP in vascular smooth muscle cells. Am J Physiol-Cell PH. 2008;295(5):C1261-70.

66. Szabo C. Hydrogen sulphide and its therapeutic potential. Nat rev. 2007 Nov; 6(11):917-35.

67. Olson KR. The Therapeutic Potential of Hydrogen Sulfide: Separating Hype from Hope. Am J Physiol Regul Integr Comp Physiol. 2011 Aug;301(2):R297-312

68. Van der Sterren S, Kleikers P, Zimmermann LJ, Villamor E. Vasoactivity of the gasotransmitters hydrogen sulfide and carbon monoxide in the chicken ductus arteriosus. Am J Physiol Regul Integr Comp Physiol. 2011 Oct;301(4):R1186-98. 
69. Baragatti B, Ciofini E, Sodini D, Luin S, Scebba F, Coceani F. Hydrogen sulfide in the mouse ductus arteriosus: a naturally occurring relaxant with potential EDHF function. Am J Physiol Heart Circ Physiol. 2013 Apr 1;304(7):H927-34.

70. Radermacher KA, Wingler K, Langhauser F, Altenhofer S, Kleikers P, Hermans JJ, et al. Neuroprotection After Stroke by Targeting NOX4 As a Source of Oxidative Stress. Antioxid Redox Signal. 2012 Oct 16.

71. Bowler RP, Crapo JD. Oxidative stress in allergic respiratory diseases. The Journal of allergy and clinical immunology. 2002 Sep;110(3):349-56.

72. Martindale JL, Holbrook NJ. Cellular response to oxidative stress: signaling for suicide and survival. J Cell Physiol. 2002 Jul;192(1):1-15.

73. Saugstad OD. Oxidative stress in the newborn--a 30-year perspective. Biol Neonate. 2005;88(3):228-36.

74. Davis JM, Auten RL. Maturation of the antioxidant system and the effects on preterm birth. Semin Fetal Neonatal Med. Aug;15(4):191-5.

75. Georgeson GD, Szony BJ, Streitman K, Varga IS, Kovacs A, Kovacs L, et al. Antioxidant enzyme activities are decreased in preterm infants and in neonates born via caesarean section. Eur J Obstet Gynecol Reprod Biol. 2002 Jul 10;103(2):136-9.

76. Richir MC, Siroen MP, van Elburg RM, Fetter WP, Quik F, Nijveldt RJ, et al. Low plasma concentrations of arginine and asymmetric dimethylarginine in premature infants with necrotizing enterocolitis. Brit J Nutr. 2007 May;97(5):906-11.

77. Perrone S, Negro S, Tataranno ML, Buonocore G. Oxidative stress and antioxidant strategies in newborns. J Matern Fetal Neonatal Med. Oct;23 Suppl 3:63-5.

78. Saugstad OD. Oxygen radical disease in neonatology. Semin Neonatol. 1988;3(3):229-38.

79. Jahn U, Galano JM, Durand T. Beyond prostaglandins--chemistry and biology of cyclic oxygenated metabolites formed by free-radical pathways from polyunsaturated fatty acids. Angew Chem Int Ed Engl. 2008;47(32):5894-955.

80. Morrow JD, Awad JA, Boss HJ, Blair IA, Roberts LJ, 2nd. Non-cyclooxygenasederived prostanoids (F2-isoprostanes) are formed in situ on phospholipids. Proc Natl Acad Sci U S A. 1992 Nov 15;89(22):10721-5.

81. Janssen LJ, Catalli A, Helli P. The pulmonary biology of isoprostanes. Antioxid Redox Signal. 2005 Jan-Feb;7(1-2):244-55.

82. Belik J, Gonzalez-Luis GE, Perez-Vizcaino F, Villamor E. Isoprostanes in fetal and neonatal health and disease. Free Radic Biol Med. 2010 Jan 15;48(2):177-88.

83. Comporti M, Signorini C, Leoncini S, Buonocore G, Rossi V, Ciccoli L. Plasma F2-isoprostanes are elevated in newborns and inversely correlated to gestational age. Free Radic Biol Med. 2004 Sep 1;37(5):724-32.

84. Delanty N, Reilly M, Pratico D, FitzGerald DJ, Lawson JA, FitzGerald GA. 8-Epi PGF2 alpha: specific analysis of an isoeicosanoid as an index of oxidant stress in vivo. Br J Clin Pharmacol. 1996 Jul;42(1):15-9.

85. Morrow JD, Roberts LJ, 2nd. The isoprostanes. Current knowledge and directions for future research. Biochem Pharmacol. 1996 Jan 12;51(1):1-9. 
86. Signorini C, Perrone S, Sgherri C, Ciccoli L, Buonocore G, Leoncini S, et al. Plasma esterified F2-isoprostanes and oxidative stress in newborns: role of nonproteinbound iron. Pediatr Res. 2008 Mar;63(3):287-91.

87. van der Sterren S, Villamor E. Contractile effects of 15-E2t-isoprostane and 15F2t-isoprostane on chicken embryo ductus arteriosus. Comp Biochem Physiol A Mol Integr Physiol. 2011 Aug;159(4):436-44.

88. Chen JX, O'Mara PW, Poole SD, Brown N, Ehinger NJ, Slaughter JC, et al. Isoprostanes as physiological mediators of transition to newborn life: novel mechanisms regulating patency of the term and preterm ductus arteriosus. Pediatr Res. 2012 Aug; 72(2):122-8.

89. Gruzdev A, Nguyen M, Kovarova M, Koller BH. PGE2 through the EP4 receptor controls smooth muscle gene expression patterns in the ductus arteriosus critical for remodeling at birth. Prostag Oth Lipid M. 2012 Mar;97(3-4):109-19.

90. Leonhardt A, Glaser A, Wegmann M, Schranz D, Seyberth H, Nusing R. Expression of prostanoid receptors in human ductus arteriosus. $\mathrm{Br} \mathrm{J}$ Pharmacol. 2003 Feb;138(4):655-9.

91. Loftin CD, Trivedi DB, Tiano HF, Clark JA, Lee CA, Epstein JA, et al. Failure of ductus arteriosus closure and remodeling in neonatal mice deficient in cyclooxygenase-1 and cyclooxygenase-2. P Natl Acad Sci USA. 2001 Jan 30;98(3):1059-64.

92. Yokota T, Shiraishi R, Aida T, Iwai K, Liu NM, Yokoyama U, et al. Thromboxane $\mathrm{A}(2)$ receptor stimulation promotes closure of the rat ductus arteriosus through enhancing neointima formation. PloS one. 2014;9(4):e94895.

93. Yokota T, Aida T, Ichikawa Y, Fujita T, Yokoyama U, Minamisawa S. Low-dose thromboxane A2 receptor stimulation promotes closure of the rat ductus arteriosus with minimal adverse effects. Pediatr Res. 2012 Aug;72(2):129-36.

94. Yuhki K, Kojima F, Kashiwagi H, Kawabe J, Fujino T, Narumiya S, et al. Roles of prostanoids in the pathogenesis of cardiovascular diseases: Novel insights from knockout mouse studies. Pharmacol Therapeut. 2011 Feb;129(2):195-205.

95. Di Renzo GC, Rosati A, Sarti RD, Cruciani L, Cutuli AM. Does fetal sex affect pregnancy outcome? Gend Med. 2007 Mar;4(1):19-30.

96. Drevenstedt GL, Crimmins EM, Vasunilashorn S, Finch CE. The rise and fall of excess male infant mortality. P Natl Acad Sci USA. 2008 Apr 1;105(13):5016-21.

97. Stevenson DK, Verter J, Fanaroff AA, Oh W, Ehrenkranz RA, Shankaran S, et al. Sex differences in outcomes of very low birthweight infants: the newborn male disadvantage. Arch Dis Child Fetal Neonatal Ed. 2000 Nov;83(3):F182-5.

98. Khali RA. Sex hormones as potential modulators of vascular function in hypertension. Hypertension. 2005 Aug;46(2):249-54.

99. Weir EK, Lopez-Barneo J, Buckler KJ, Archer SL. Acute oxygen-sensing mechanisms. N Engl J Med. 2005 Nov 10;353(19):2042-55.

100. Moral-Sanz J, Gonzalez T, Menendez C, David M, Moreno L, Macias A, et al. Ceramide inhibits $\mathrm{Kv}$ currents and contributes to TP-receptor-induced vasoconstriction in rat and human pulmonary arteries. Am J Physiol-Cell PH. 2011 Jul;301(1):C186-94. 
101. Frazziano G, Moreno L, Moral-Sanz J, Menendez C, Escolano L, Gonzalez C, et al. Neutral sphingomyelinase, NADPH oxidase and reactive oxygen species. Role in acute hypoxic pulmonary vasoconstriction. J Cell Physiol. 2011 Oct;226(10):2633-40.

102. Cogolludo A, Moreno L, Frazziano G, Moral-Sanz J, Menendez C, Castaneda J, et al. Activation of neutral sphingomyelinase is involved in acute hypoxic pulmonary vasoconstriction. Cardiovasc Res. 2009 May 1;82(2):296-302.

103. Kleikers PW, Wingler K, Hermans JJ, Diebold I, Altenhofer S, Radermacher KA, et al. NADPH oxidases as a source of oxidative stress and molecular target in ischemia/ reperfusion injury. J Mol Med (Berl). 2012 Oct 23.

104. Altenhofer S, Kleikers PW, Radermacher KA, Scheurer P, Rob Hermans JJ, Schiffers $P$, et al. The NOX toolbox: validating the role of NADPH oxidases in physiology and disease. Cell Mol Life Sci. 2012 Jul;69(14):2327-43.

105. Gupte SA, Wolin MS. Relationships between vascular oxygen sensing mechanisms and hypertensive disease processes. Hypertension. 2012 Aug;60(2): 269-75.

106. Perez-Vizcaino F, Cogolludo A, Moreno L. Reactive oxygen species signaling in pulmonary vascular smooth muscle. Respir Physiol Neurobiol. 2010 Dec 31;174(3): 212-20.

107. Neo BH, Kandhi S, Ahmad M, Wolin MS. Redox regulation of guanylate cyclase and protein kinase $G$ in vascular responses to hypoxia. Respir Physiol Neurobiol. 2010 Dec 31;174(3):259-64.

108. Archer SL, Reeve HL, Michelakis E, Puttagunta L, Waite R, Nelson DP, et al. $\mathrm{O} 2$ sensing is preserved in mice lacking the gp91 phox subunit of NADPH oxidase. P Natl Acad Sci USA. 1999 Jul 6;96(14):7944-9.

109. Wind S, Beuerlein K, Eucker T, Muller H, Scheurer P, Armitage ME, et al. Comparative pharmacology of chemically distinct NADPH oxidase inhibitors. $\mathrm{Br} \mathrm{J}$ Pharmacol. 2010 Oct;161(4):885-98.

110. Rivera J, Sobey CG, Walduck AK, Drummond GR. Nox isoforms in vascular pathophysiology: insights from transgenic and knockout mouse models. Redox rep: communications in free radical research. 2010;15(2):50-63.

111. Ambasta RK, Kumar P, Griendling KK, Schmidt HH, Busse R, Brandes RP. Direct interaction of the novel Nox proteins with p22phox is required for the formation of a functionally active NADPH oxidase. J Biol Chem. 2004 Oct 29;279(44):45935-41.

112. Gulbins E, Li PL. Physiological and pathophysiological aspects of ceramide. Am J Physiol Regul Integr Comp Physiol. 2006 Jan;290(1):R11-26.

113. Liu B, Andrieu-Abadie N, Levade T, Zhang P, Obeid LM, Hannun YA. Glutathione regulation of neutral sphingomyelinase in tumor necrosis factor-alphainduced cell death. J Biol Chem. 1998 May 1;273(18):11313-20.

114. Waypa GB, Schumacker PT. Hypoxia-induced changes in pulmonary and systemic vascular resistance: where is the $\mathrm{O} 2$ sensor? Respir Physiol Neurobiol. Dec 31;174(3):201-11. 
115. Rathore R, Zheng YM, Niu CF, Liu QH, Korde A, Ho YS, et al. Hypoxia activates NADPH oxidase to increase [ROS]i and [Ca2+]i through the mitochondrial ROSPKCepsilon signaling axis in pulmonary artery smooth muscle cells. Free Radic Biol Med. 2008 Nov 1;45(9):1223-31.

116. Antico Arciuch VG, Elguero ME, Poderoso JJ, Carreras MC. Mitochondrial regulation of cell cycle and proliferation. Antioxid Redox Signal. 2012 May 15;16(10): 1150-80.

117. Nakai A, Taniuchi Y, Asakura H, Oya A, Yokota A, Koshino T, et al.

Developmental changes in mitochondrial activity and energy metabolism in fetal and neonatal rat brain. Dev Brain Res. 2000;121(1):67-72

118. Porter GA, Jr., Hom J, Hoffman D, Quintanilla R, de Mesy Bentley K, Sheu SS. Bioenergetics, mitochondria, and cardiac myocyte differentiation. Prog Pediatr Cardiol. 2011 May;31(2):75-81.

119. Prieur B, Bismuth J, Delaval E. Effects of adrenal steroid hormones on mitochondrial maturation during the late fetal period. Eur J Biochem. 1998 Mar 1;252(2): 194-9.

120. Hong Z, Kutty S, Toth PT, Marsboom G, Hammel JM, Chamberlain C, et al. Role of dynamin-related protein 1 (Drp1)-mediated mitochondrial fission in oxygen sensing and constriction of the ductus arteriosus. Circ Res. 2013 Mar 1;112(5):802-15.

121. Jobe AH. Prenatal Corticosteroids: A Neonatologist's Perspective. NeoReviews. 2006 May 1, 2006;7(5):e259-e67.

122. Echtler K, Stark K, Lorenz M, Kerstan S, Walch A, Jennen L, Rudelius M, Seidl S, Kremmer E, Emambokus NR, von Bruehl ML, Frampton J, Isermann B, GenzelBoroviczény O, Schreiber C, Mehilli J, Kastrati A, Schwaiger M, Shivdasani RA, Massberg S. Platelets contribute to postnatal occlusion of the ductus arteriosus. Nat Med. $2010 ; 16(1): 75-82$.

123. Clyman R, Chemtob S. Vessel remodeling in the newborn: platelets fill the gap. Nat Med. 2010;16:33-5

124. Alyamac Dizdar E, Ozdemir R, Nur Sari F, Yurttutan S, Gokmen T, Erdeve O, et al. Low platelet count is associated with ductus arteriosus patency in preterm newborns. Early Hum Dev. 2012, 88(10): 813-816.

125. Dani C, Poggi C, Fontanelli G. Relationship between Platelet Count and Volume and Spontaneous and Pharmacological Closure of Ductus Arteriosus in Preterm Infants. Am J Perinat. 2013, 30(5): 359-364.

126. Fujioka K, Morioka I, Miwa A, Morikawa S, Shibata A, Yokoyama N, et al. Does thrombocytopenia contribute to patent ductus arteriosus? Nat Med. 2011, 17(1): 29-30; author reply 30-21.

127. Shah NA, Hills NK, Waleh N, McCurnin D, Seidner S, Chemtob S, et al. Relationship between circulating platelet counts and ductus arteriosus patency after indomethacin treatment. J Pediatr. 2011, 158(6): 919-923. 
128. Sallmon H, Weber SC, Huning B, Stein A, Horn PA, Metze BC, et al. Thrombocytopenia in the first 24 hours after birth and incidence of patent ductus arteriosus. Pediatrics. 2012, 130(3): e623-630.

129. Chen YY, Wang HP, Chang JT, Chiou YH, Huang YF, Hsieh KS, et al. Perinatal factors in patent ductus arteriosus in very low-birthweight infants. Pediatr Int: official journal of the Japan Pediatric Society. 2014, 56(1): 72-76.

130. Bas-Suárez M, González-Luis G, Saavedra P, Villamor E. Platelet counts in the first seven days of life and patent ductus arteriosus in preterm very low birth weight infants. Neonatology. 2014;106:188-94 doi:

131. Simon SR, van Zogchel L, Bas-Suárez P, Cavallaro G, Clyman RI, Villamor E. Platelet counts and patent ductus arteriosus in preterm infants: a meta-analysis.

Neonatology. 2015 Jul 7;108(2):143-151.

132. Williams MS, Weiss EJ, Sabatine MS, Simon DI, Bahou WF, Becker LC, Parise LV, Dauerman HL, French PA, Smyth SS, and Becker RC. Genetic regulation of platelet receptor expression and function: application in clinical practice and drug development. Arterioscl Throm Vas. 2010;30: 2372-2384.

133. Brass S. Cardiovascular biology. Platelets and proteases. Nature. $2001 \mathrm{Sep}$ 6;413(6851):26-7.

134. Hirano K, Kanaide $\mathrm{H}$. Role of protease-activated receptors in the vascular system. J Atheroscler Thromb. 2003;10(4):211-25.

135. Vogel SM, Gao X, Mehta D, Ye RD, John TA, Andrade-Gordon P, et al. Abrogation of thrombin-induced increase in pulmonary microvascular permeability in PAR-1 knockout mice. Physiol Genomics. 2000 Dec 18;4(2):137-45.

136. Hammerman C, Bin-Nun A, Markovitch E, Schimmel MS, Kaplan M, Fink D. Ductal closure with paracetamol: a surprising new approach to patent ductus arteriosus treatment. Pediatrics. 2011 Dec;128(6):e1618-21.

137. Oncel MY, Yurttutan S, Degirmencioglu H, Uras N, Altug N, Erdeve O, et al. Intravenous paracetamol treatment in the management of patent ductus arteriosus in extremely low birth weight infants. Neonatology. 2013;103(3):166-9.

138. Yurttutan S, Oncel MY, Arayici S, Uras N, Altug N, Erdeve O, et al. A different first-choice drug in the medical management of patent ductus arteriosus: oral paracetamol. J Matern Fetal Neonatal Med. 2013 May;26(8):825-7.

139. Oncel MY, Yurttutan S, Uras N, Altug N, Ozdemir R, Ekmen S, et al. An alternative drug (paracetamol) in the management of patent ductus arteriosus in ibuprofen-resistant or contraindicated preterm infants. Arch Dis Child Fetal Neonatal Ed. 2012 May 18.

140. Allegaert K, Anderson B, Simons S, van Overmeire B. Paracetamol to induce ductus arteriosus closure: is it valid? Arch Dis Child. 2013 Jun;98(6):462-6.

141. Ohlsson A, Shah PS. Paracetamol (acetaminophen) for patent ductus arteriosus in preterm and/or low-birth-weight infants. Cochrane Database Syst Rev. 2015 Mar 11;3:CD010061. 
142. Lucas R, Warner TD, Vojnovic I, Mitchell JA. Cellular mechanisms of acetaminophen: role of cyclo-oxygenase. Faseb J. 2005 Apr;19(6):635-7.

143. El-Khuffash A, Jain A, Corcoran D, Shah PS, Hooper CW, Brown N, et al. Efficacy of paracetamol on patent ductus arteriosus closure may be dose dependent: evidence from human and murine studies. Pediatr Res. 2014 Jun 18.

144. Reese J, Veldman A, Shah L, Vucovich M, Cotton RB. Inadvertent relaxation of the ductus arteriosus by pharmacologic agents that are commonly used in the neonatal period. Semin Perinatol. 2010 Jun;34(3):222-30.

145. Dias-Guichot Y, Verver E, Van Der Sterren S, Villamor E. Magnesium Sulfate Evokes Endothelium-Independent Relaxation in the Chicken Ductus Arteriosus. Pediatr Res. 2011;70(S5):285-285.

146. Jacobs S, Hunt R, Tarnow-Mordi W, Inder T, Davis P. Cooling for newborns with hypoxic ischaemic encephalopathy. Cochrane Database Syst Rev. 2007 (4):CD003311.

147. Eicher DJ, Wagner CL, Katikaneni LP, Hulsey TC, Bass WT, Kaufman DA, et al. Moderate hypothermia in neonatal encephalopathy: safety outcomes. Pediatr Neurol. 2005 Jan;32(1):18-24.

148. Cavallaro G, Bijker E, Strackx E, Vles JS, Gavilanes D, Villamor E. Age-related changes and effects of mild hypothermia on carotid artery reactivity in newborn rats. CNS Neurol Disord Drug Targets. 2015;14(1):91-101. 
Chapter 9

Samenvatting 


\subsection{Introductie}

De ductus arteriosus (DA) is een vat dat tijdens de foetale periode een verbinding vormt tussen de longslagader en de aorta. De longen van een foetus zijn nog niet functioneel, gasuitwisseling vindt immers plaats in de placenta. Het zuurstofrijke bloed dat richting de longen gepompt wordt, wordt grotendeels via de DA naar de aorta geleid zodat het bloed sneller bij alle kritische organen komt. De DA is gevoelig voor zuurstof en in de foetus wordt de DA ontspannen door de relatief zuurstofarme omgeving. Bij de geboorte zorgen gelijktijdige uitzetting van de longvaten en samentrekken van de DA (als reactie op een hogere zuurstofconcentratie in het bloed nadat de pasgeborene is beginnen adem te halen) ervoor dat het bloed vanuit de rechter hartkamer nu wél naar de longen wordt gevoerd. Falen van het samentrekkingen en sluiten van de DA na de geboorte is een veel voorkomende complicatie bij te vroeg geboren kinderen en levert nog steeds uitdagingen wat betreft diagnostiek, evaluatie en behandelingsopties.

De kip als model voor de ontwikkelingsbiologie van de ductus arteriosus De Amerikaanse filosoof Marx Wartofsky zei: "alles kan een model zijn voor iets anders! Oftewel: willekeurig welke twee dingen in het universum je neemt, er is altijd een eigenschap die ze delen". Modellen hoeven dan ook niet in alle eigenschappen overeengekomen met dat waarvan zij een model zijn. Soms is het zelfs essentieel dat deze verschillen er zijn. Kippen zijn warmbloedige gewervelde dieren, die biologisch gezien veel overeenkomen met zoogdieren. De kip is een ideaal model voor het onderzoeken van de eigenschappen van de DA en andere bloedvaten. Bovendien is het relatief eenvoudig om de omstandigheden waarin het kippenembryo zich ontwikkelt aan te passen, omdat deze ontwikkeling plaatsvindt buiten het moederdier. Zo is het bijvoorbeeld mogelijk om situaties met verminderde aanvoer van zuurstof of voedingsstoffen na te bootsen, of de invloed van bijvoorbeeld medicatie op de embryogenese te bestuderen.

\subsection{Dit proefschrift}

In hoofdstuk 1 word beschreven wat de overeenkomsten en verschillen zijn tussen de DA in zoogdieren en in kippen. Daarnaast wordt een overzicht gegeven van wat bekend was over de eigenschappen van de DA. De belangrijkste overeenkomst en conclusie is, dat de DA in kippen net als in 
zoogdieren samentrekt als reactie op zuurstof en daarmee als model kan dienen om dit verder te bestuderen.

In hoofdstuk 2 wordt dieper ingegaan op de zuurstof-respons van de DA en laten we zien dat het mechanisme van zuurstof sensor, mediator en effector, waarvan in zoogdieren al was aangetoond dat dit leidt tot sluiten van de DA, ook in ons kippenembryo-model aanwezig is. Dit mechanisme, met de mitochondria als zuurstofsensor, waterstofperoxide als mediator en kaliumkanalen als effector van de zuurstofrespons, is dus al vroeg in de evolutie aanwezig geweest en is in de loop van de tijd blijven bestaan.

Ook hebben we gezien dat deze componenten alledrie voldoende uitgerijpt moeten zijn om te leiden tot voldoende contractie van de DA en dat falen van sluiten van de ductus dus niet te wijten is aan één van drie.

In hoofdstuk 3 en 4 onderzoeken we wat de invloed van minder of meer zuurstof (hypoxie of hyperoxie) is op de ontwikkeling van het kippenembryo en in het bijzonder op de eigenschappen van de DA. Alhoewel er in beide gevallen wel veranderingen worden gezien, leidt dit uiteindelijk niet tot een verminderd vermogen tot sluiten van de DA.

In hoofdstuk 5 hebben we de invloed van de gassen waterstofsulfide en koolstofmonoxide onderzocht. Er zijn verschillende gassen, waarvan al bekend was dat deze invloed hebben op het samentrekken of ontspannen van de DA. Voorbeelden hiervan zijn zuurstof, koolstofdioxide en stikstofoxide. Van waterstofsulfide was al bekend dat het invloed heeft op bloedvaten, maar het effect ervan op de DA was nog nooit onderzocht. In ons model blijkt dat zowel waterstofsulfide als koolstofmonoxide de DA doet ontspannen, wat ertoe kan leiden dat er onvoldoende constrictie ontstaat. Bij beide stoffen kon echter niet aangetoond worden dat deze in voldoende mate door het vat zelf geproduceerd worden om sluiten van de DA te voorkomen.

In hoofdstuk 6 worden isoprostanen besproken, stoffen die vrijkomen bij 'oxidatieve stress' zoals bijvoorbeeld ook bij geboorte plaats vindt. Deze isoprostanen werden al gezien als markers van de mate van oxidatieve stress, maar blijken zelf ook effect te hebben op bloedvaten. Onze resultaten laten zien dat de DA samentrekt in aanwezigheid van isoprostanen, wat suggereert dat deze na de geboorte een rol spelen in het sluiten van de DA. 
De invloed van geslacht en geslachtshormonen worden besproken in hoofdstuk 7. Op de couveuse-afdeling wordt gezien dat jongetjes een slechtere prognose hebben dan meisjes wat betreft het optreden van voorkomende complicaties, waaronder ook het niet sluiten van de DA. Desondanks zien we in onze studies geen verschillen in de reactiviteit van de DA tussen de twee geslachten, alhoewel het toedienen van oestrogeen wel zorg voor ontspanning van de DA.

Bovenstaande bevindingen hebben bijgedragen aan een beter begrip van de reactiviteit en nodigen uit tot verder onderzoek naar de biologische achtergrond hiervan en eventuele nieuwe behandelingsmogelijkheden. 
Chapter 10

\section{Valorisation}




\section{8}




\subsection{Relevance}

Failure of DA closure is a common complication in very preterm infants that can have significant clinical consequences and still presents challenges in terms of diagnosis, assessment and treatment options [1]. It is generally assumed that prolonged patency of the DA (PDA) is associated with several comorbidities, such as necrotizing enterocolitis, intracranial hemorrhage, pulmonary edema/ hemorrhage, broncho-pulmonary dysplasia and retinopathy, and has a negative impact on outcome in such infants [1,2]. Nevertheless, there is an ongoing debate about the thresholds and strategies for PDA treatment. Adverse effects of pharmacological and surgical therapies must be weighed against conflicting information on which adverse outcomes can be attributed to PDA and unclear determinations of whether PDA closure alleviates those outcomes. Yet daily rounds in the neonatal intensive care unit frequently include bedside deliberations over management of preterm infants whose PDA is considered problematic [3]. In summary, PDA in preterm infants has been defined as a continuing conundrum -i.e., a question or problem having only a conjectural answer- for the neonatologist [4]. Needless to say, a more profound understanding of the (patho)biology of the DA, and the mechanisms that regulate its vascular tone, could have clinical implications and lead to better management options and better neonatal outcome.

Significant progress in our understanding of the DA physiology and pathophysiology has been achieved with the use of animal models, including the sheep, the rabbit, the mouse, the rat, or primates [1,2]. However, mammalian models are complex because the fetal/placental circulation has to be exposed to intervention only through complex surgery and experimental manipulations affect both the mother and the fetus. Therefore, there is a need for additional models, addressing these limitations [2].The chicken (Gallus gallus) embryo represents an excellent model for investigating developmental physiology of the cardiovascular system [5]. Chicken embryos have a mammalian-like circulation, with an extraembryonic circuit involved in the gas exchange (the chorioallantois), analogous to the placenta and they maintain bilaterally developed DA [6]. As the chicken embryo develops outside the mother, the number of experimental animals is divided by two, and effects of external stresses on cardiovascular development can be studied without interferences of maternal hormonal, metabolic, or hemodynamic alterations. 


\subsection{Innovation}

With the present project, we aimed to implement and extend the utilization of the chicken embryo as a model for developmental vascular biology. Although probably more is known about the descriptive embryology, teratology, and experimental embryology of the chicken embryo than of any other model organism, developmental changes in the reactivity of the chicken DA had not been studied until our recent investigation [7]. In fact, the publication of our findings has already attracted the attention of other investigators in the field of DA developmental biology (see reference [2]). The numerous groups of investigators that are currently using expensive, aggressive, and technically complex mammalian-based experimental models to study developmental vascular biology would benefit from a model characterized by its tractability for experimental manipulation, its rich history in developmental biology, and a relatively short incubation time. If the similarities that we have already observed between the chicken and the mammalian and human DA are confirmed, then a new model will be available for translational studies for the human DA and, moreover, for the biology of vascular $\mathrm{O}_{2}$ sensing in vivo and in vitro. In addition, investigators interested in the effects of prenatal insults in development or in the pathophysiology of human diseases would benefit from a model in which insults (such as hypoxia, malnutrition or exposure to drugs or toxics) are easily performed and are not interfered by maternal influences.

The use of animal subjects is fundamental to the advancement of biomedical research and practice, at least until viable replacements are found. Equally important are the ethical guidelines and welfare protocols that shape the conduct of such research. Animal welfare guidelines and legislation still emphasise Russell and Burch's 'three Rs': reduction (of the number of animals used), refinement (of testing procedures to minimise suffering), and replacement (of animal models with alternatives) [7].

Nevertheless, there are those with the strongly held belief that it is morally indefensible for mankind to subject animals to experimental procedures that cause any degree of discomfort, as an animal is unable to provide informed consent and does not benefit directly or indirectly from the studies. These guidelines and protocols mark the interface between the experimental needs of medical and scientific researchers and the socially acceptable degree of suffering the experimental subjects should endure and/or consent to for research benefits. However, there is a marked distinction between human 
phase regulations, which focus on issues of patient recruitment and consent, and the laboratory animal phase that focuses on the 'three Rs' of reduction, refinement, and replacement.

In conclusion, this thesis adds to the body of knowledge about DA vasoreactivity, furthering our understanding of it's pathophysiology. This bring us one step closer to developing better treatment strategies for PDA in a vulnerable group of patients - the very preterm and/or growth restricted infants at risk for several associated neonatal comorbidities - and thus contributing towards a better outcome. 


\section{References}

1. Clyman, R.I., Mechanisms regulating the ductus arteriosus. Biol Neonate, 2006. 89(4):

p. 330-5.

2. Sutendra, G. and E.D. Michelakis, The chicken embryo as a model for ductus arteriosus developmental biology: cracking into new territory. Am J Physiol Regul Integr Comp Physiol, 2007. 292(1): p. R481-4.

3. Reese J, Laughon MM. The Patent Ductus Arteriosus Problem: Infants Who Still Need Treatment. J Pediatr. 2015 Nov;167(5):954-6. doi:10.1016/j.jpeds.2015.08.023. Epub 2015 Aug 24. PubMed PMID: 26316368.

4. Evans N. Preterm patent ductus arteriosus: A continuing conundrum for the neonatologist? Semin Fetal Neonatal Med. 2015 Aug;20(4):272-7. doi: 10.1016/j.siny. 2015.03.004. Epub 2015 Mar 25. Review. PubMed PMID: 25818393. 3.

5. Ruijtenbeek, K., J.G. De Mey, and C.E. Blanco, The chicken embryo in developmental physiology of the cardiovascular system: a traditional model with new possibilities. Am J Physiol Regul Integr Comp Physiol, 2002. 283(2): p. R549-50; author reply R550-1.

6. Agren, P., et al., Ontogeny of chicken ductus arteriosus response to oxygen and vasoconstrictors. Am J Physiol Regul Integr Comp Physiol, 2007. 292(1): p. R485-96.

7. Russell WMS, Burch RL 1959. The Principles of Humane Experimental Technique. London: Methuen \& Co. Ltd. [Reissued: 1992, Universities Federation for Animal Welfare, Herts, England.] 


\section{Dankwoord}

(Acknowledgements) 
Mijn proefschrift is klaar, the end of an era. Naast trots op het resultaat ben ik dankbaar voor iedereen die heeft bijgedragen aan het tot stand komen ervan. Voor iedereen een welgemeend: hartelijk dank, mede dankzij jullie sta ik nu hier!

Een aantal mensen wil ik expliciet noemen:

Geen promotie zonder promotores: prof.dr. Luc Zimmermann en prof. dr. Angel Cogolludo. Beste Luc, bedankt voor je aanwezigheid en steun gedurende de loop van dit traject. Dear Angel, I have enjoyed the (short) time we worked at the lab together, I admire your energy and insight and very much appreciated your feedback.

Vooral ook geen promotie zonder co-promotor: dr. Eduardo Villamor.

Beste Eduardo, vanaf het moment dat ik bij je aanklopte voor een onderzoeksstage werd ik gegrepen door je enthousiasme, je energie en je niet aflatende inzet. Die onderzoeksstage groeide uit tot dit promotietraject waarin naast wetenschappelijke ontwikkeling ook ruimte was voor het delen van klinische- en levenslessen. Bedankt voor de ruimte die je me gaf en je betrokkenheid die ik heb mogen ervaren!

De leden van de beoordelingscommissie, bestaande uit prof.dr. Boris Kramer (voorzitter), prof.dr. Aalt Bast, prof.dr. Ronald Clyman, prof.dr. Tammo Delhaas, en prof.dr. Irwin Reiss, wil ik hartelijk bedanken voor het lezen en beoordelen van mijn proefschrift, ditzelfde geldt voor de leden van de corona.

Mijn dank gaat ook uit naar de co-auteurs en hun bijdragen aan de artikelen die ten grondslag liggen aan dit proefschrift. Anne dank ik daarnaast, zoals beloofd, voor het rennen!

Alle andere collega's op het lab door de jaren heen: Bea, Coen, Elke, Jennifer, Leon, Lilian, Nico, Pia, Tim, en vele anderen; zonder jullie expertise, steun en gezelligheid was het lang niet zo'n mooie ervaring geweest.

Steeds belangstellend en waar mogelijk faciliterend waren de de kinderartsen in Sittard, Heerlen en Maastricht. Als semi-arts, ANIOS en AIOS is bij jullie tevens de basis gelegd voor mijn opleiding. Ik dank jullie voor het stimuleren van mijn ontwikkeling tot kinderarts. 
Dank ook aan alle vrienden, familieleden en collega's die steeds met een luisterend oor, praktische adviezen of een kop koffie een steun zijn geweest. Met name ook Ruud en Chris, zonder wie ik niet zou kunnen doen wat ik doe.

Mijn paranimfen:

Lilian, jouw expertise op het lab loodste me door alle (praktische) problemen heen en je expertise 'oppe Ruiver' was minstens zo waardevol. Je bent steeds een rots in de branding geweest en ik ben blij dat jij vandaag aan mijn zijde staat.

Ger, mijn broer. Nog zo'n rots in de branding, evenals zus Ingrid. Ik prijs me gelukkig met jullie. Ik ben blij dat ook jij vandaag aan mijn zijde staat.

Mam, mijn dankbaarheid voor jou kent geen grenzen, $\mathrm{X}$.

Pap, ik weet dat je trots geweest zou zijn.

En, saving the best for last:

Maurice, Imke en Kasper, ik hou van jullie! Dank voor jullie liefde en geduld. Een dikke kus en knuffel voor jullie alledrie!

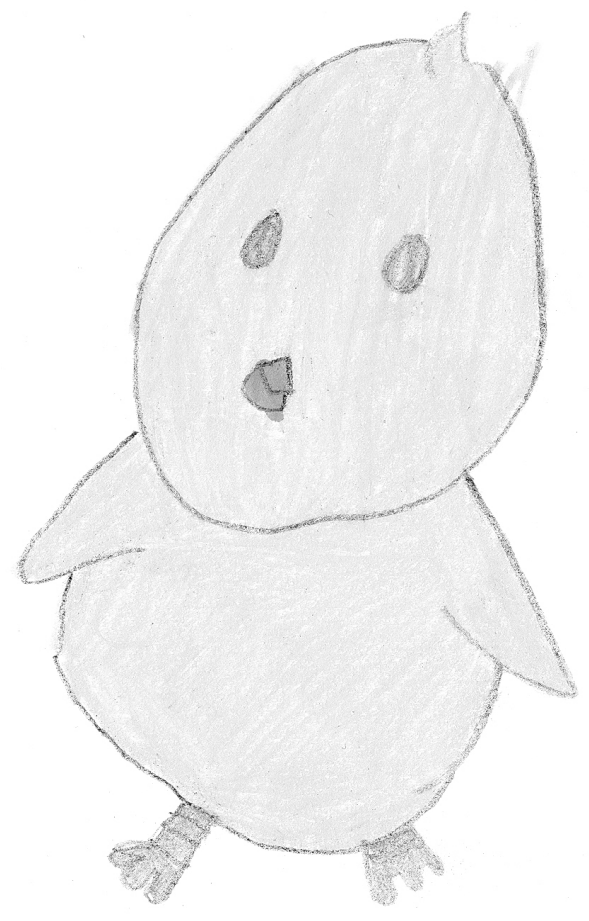




\section{Curriculum vitae en publicaties}




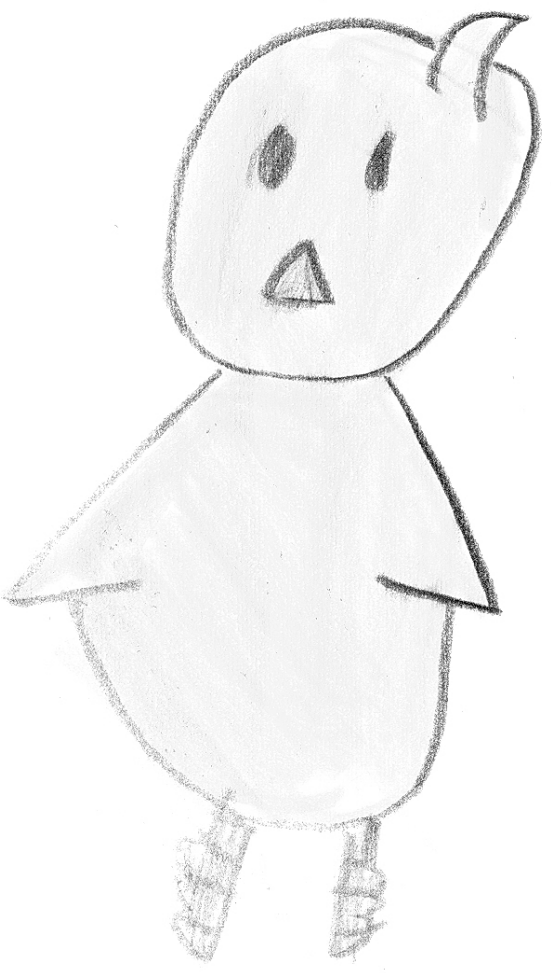

228 


\section{Curriculum vitae}

Saskia van der Sterren werd geboren op 16 april 1981 in Heerlen en groeide op in Brunssum. In 1998 behaalde zij haar gymnasiumdiploma aan het SintJanscollege in Hoensbroek. Vervolgens studeerde ze gezondheidswetenschappen, psychologie en natuurwetenschappen, om in 2003 te beginnen met de studie geneeskunde aan de Universiteit van Maastricht, waar ze in 2009 haar artsdiploma behaalde. Tijdens deze studie startte zij met haar promotieonderzoek onder supervisie van dr. Eduardo Villamor, waaraan ze na haar afstuderen een jaar voltijds kon werken dankzij een Kootstra Talent Fellowship. Vanaf december 2010 startte zij haar klinische carrière als ANIOS kindergeneeskunde in achtereenvolgens het Atrium Medisch Centrum in Heerlen en het Orbis Medisch Centrum in Sittard-Geleen. Vanaf januari 2013 is Saskia begonnen met de opleiding tot kinderarts in het Atrium Medisch Centrum in Heerlen (opleider: Dr. J.O.O. Busari) en het Maastricht Universitair Medisch Centrum (opleider: Dr. A.L.M. Mulder). Saskia woont in Brunssum met haar man Maurice en hun kinderen, Imke en Kasper.

Saskia van der Sterren was born on April 6th, 1981 in Heerlen and grew up in Brunssum. In 1998 she graduated from high school at the St.-Janscollege in Hoensbroek. Subsequently she studied health sciences, psychology and natural sciences, and started Medical school in 2003 at the Maastricht University where she obtained her degree in 2009. During this period she also initiated her PhD project under supervision of dr. Eduardo Villamor. After graduation, she worked full time on this project for a year, due to an awarded Kootstra Talent Fellowship. In december 2010 Saskia started her clinical career as a resident in pediatrics at the Atrium Medical Centre in Heerlen and the Orbis Medical Centre in SittardGeleen and in January 2013 she commenced her residential training in pediatrics at the Atrium Medical Centre in Heerlen (supervisor: Dr. J.O.O. Busari) and the Maastricht University Medical Centre (supervisor: Dr. A.L.M. Mulder). Saskia lives in Brunssum with her husband Maurice and their children, Imke and Kasper. 


\section{Publicaties}

1. Developmental changes in endothelium-dependent relaxation of the chicken ductus arteriosus. Agren P, van der Sterren S, Cogolludo AL, Frazziano G, de Mey JG, Blanco CE, Villamor E. J Physiol Pharmacol. 2008 Mar;59(1):55-76.

2. Developmental changes in the effects of prostaglandin $E_{2}$ in the chicken ductus arteriosus. Agren P, van der Sterren S, Cogolludo AL, Blanco CE, Villamor E. J Comp Physiol B. 2009 Feb;179(2):133-43.

3. Morphological and functional alterations of the ductus arteriosus in a chicken model of hypoxia-induced fetal growth retardation. Van der Sterren S, Agren P, Zoer B, Kessels L, Blanco CE, Villamor E. Pediatr Res. 2009 Mar;65(3):279-84.

4. Maturation of $\mathrm{O}_{2}$ sensing and signaling in the chicken ductus arteriosus. Cogolludo AL, Moral-Sanz J, van der Sterren S, Frazziano G, van Cleef AN, Menéndez C, Zoer B, Moreno E, Roman A, Pérez-Vizcaino F, Villamor E. Am J Physiol Lung Cell Mol Physiol. 2009 Oct;297(4):L619-30.

5. Effects of sex and estrogen on chicken ductus arteriosus reactivity. Flinsenberg TW, van der Sterren S, van Cleef AN, Schuurman MJ, Agren P, Villamor E. Am J Physiol Regul Integr Comp Physiol. 2010 May;298(5): R1217-24.

6. Prenatal cardiovascular shunts in amniotic vertebrates. Dzialowski EM, Sirsat T, van der Sterren S, Villamor E. Respir Physiol Neurobiol. 2011 Aug 31;178(1): 66-74.

7. Contractile effects of $15-E_{2 t}$-isoprostane and $15-F_{2 t}$-isoprostane on chicken embryo ductus arteriosus. Van der Sterren S, Villamor E. Comp Biochem Physiol A Mol Integr Physiol. 2011 Aug;159(4):436-44.

8. Vasoactivity of the gasotransmitters hydrogen sulfide and carbon monoxide in the chicken ductus arteriosus. Van der Sterren S, Kleikers P, Zimmermann LJ, Villamor E. Am J Physiol Regul Integr Comp Physiol. 2011 Oct;301(4): R1186-98.

9. Prenatal exposure to hyperoxia modifies the thromboxane prostanoid receptor-mediated response to $\mathrm{H}_{2} \mathrm{O}_{2}$ in the ductus arteriosus of the chicken embryo. Van der Sterren S, Kessels L, Perez-Vizcaino F, Cogolludo AL, Villamor E. J Physiol Pharmacol. 2014 Apr; 65(2):283-93. 